INTÉGRATIONDELA FORESTERIEET DE L'AGROFORESTERIEDANSLES PLANSD'ADAPTATION NATIONAUX

[ Directives complémentaires ] 



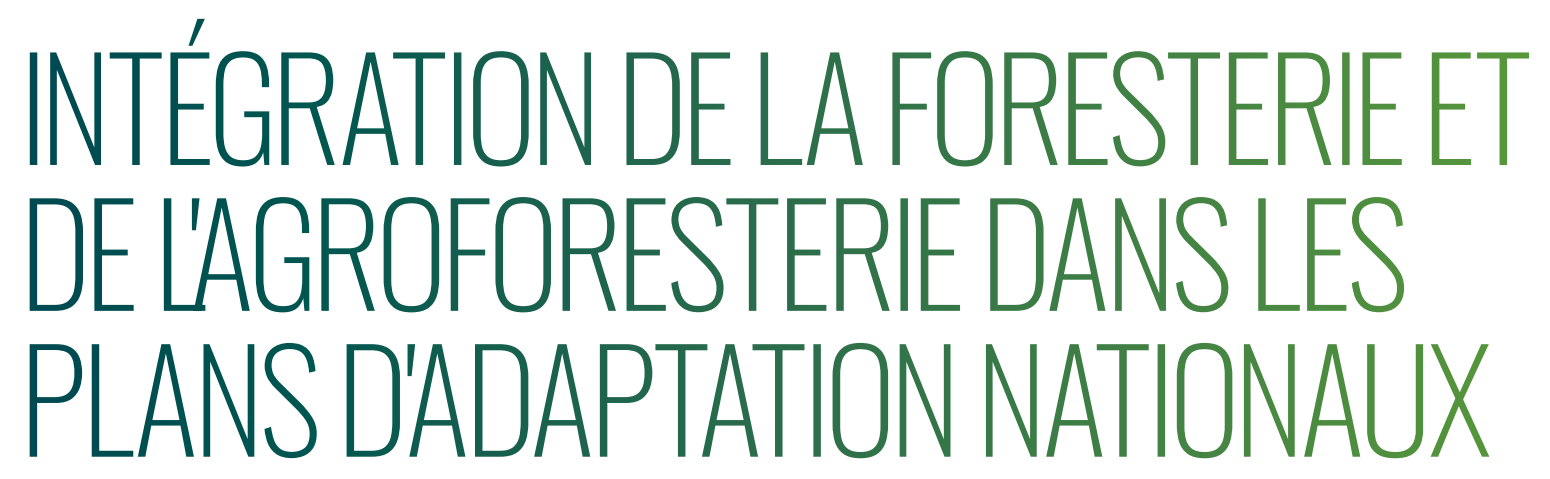

\section{[ Directives complémentaires ]}

\section{JUIN 2021}

Par Alexandre Meybeck et Vincent Gitz

Programme d'Investigation sur les forêts, les arbres et l'agroforesterie du CGIAR et

Julia Wolf et Theresa Wong

Organisation des Nations Unies pour l'alimentation et l'agriculture

Publié par I'Organisation des Nations Unies pour l'alimentation et l'agriculture et le Centre de Recherche Forestière Internationale

Rome, 2021 
Citer comme suit:

Meybeck, A., Gitz, V., Wolf, J. et Wong, T. 2021. Intégration de la foresterie et de l'agroforesterie dans les plans d'adaptation nationaux - Directives complémentaires. Bogor/Rome. FAO et CGIAR.

https://doi.org/10.4060/cb1203fr

Les appellations employées dans ce produit d'information et la présentation des données qui y figurent n'impliquent de la part de l'Organisation des Nations Unies pour l'alimentation et l'agriculture (FAO) aucune prise de position quant au statut juridique ou au stade de développement des pays, territoires, villes ou zones ou de leurs autorités, ni quant au tracé de leurs frontières ou limites. Le fait qu'une société ou qu'un produit manufacturé, breveté ou non, soit mentionné ne signifie pas que la FAO approuvé ou recommande ladite société ou ledit produit de préférence à d'autres sociétés ou produits analogues qui ne sont pas cités.

ISBN 978-92-5-134433-0

(c) FAO, 2021

\section{(c) $\underset{\mathrm{BY}}{\mathrm{NC}} \underset{\mathrm{SA}}{(0)}$}

Certains droits réservés. Cette œuvre est mise à la disposition du public selon les termes de la Licence Creative Commons Attribution-Pas d'Utilisation Commerciale-Partage dans les Mêmes Conditions 3.0 Organisations Intergouvernementales (CC BY NC SA 3.0 IGO; https://creativecommons.org/licenses/bync-sa/3.0/igo/legalcode.fr).

Selon les termes de cette licence, cette œuvre peut être copiée, diffusée et adaptée à des fins non commerciales, sous réserve que la source soit mentionnée. Lorsque l'œuvre est utilisée, rien ne doit laisser entendre que la FAO cautionne tels ou tels organisation, produit ou service. L'utilisation du logo de la FAO n'est pas autorisée. Si l'œuvre est adaptée, le produit de cette adaptation doit être diffusé sous la même licence Creative Commons ou sous une licence équivalente. Si l'œuvre est traduite, la traduction doit obligatoirement être accompagnée de la mention de la source ainsi que de la clause de non-responsabilité suivante: «La traduction n'a pas été réalisée par l'Organisation des Nations Unies pour l'alimentation et l'agriculture (FAO). La FAO n'est pas responsable du contenu ni de l'exactitude de la traduction. L'édition originale [langue] est celle qui fait foi.»

Tout litige relatif à la présente licence ne pouvant être résolu à l'amiable sera réglé par voie de médiation et d'arbitrage tel que décrit à l'Article 8 de la licence, sauf indication contraire contenue dans le présent document. Les règles de médiation applicables seront celles de l'Organisation mondiale de la propriété intellectuelle (http://www.wipo.int/amc/fr/mediation/rules) et tout arbitrage sera mené conformément au Règlement d'arbitrage de la Commission des Nations Unies pour le droit commercial international (CNUDCI).

Matériel attribué à des tiers. Il incombe aux utilisateurs souhaitant réutiliser des informations ou autres éléments contenus dans cette œuvre qui y sont attribués à un tiers, tels que des tableaux, des figures ou des images, de déterminer si une autorisation est requise pour leur réutilisation et d'obtenir le cas échéant la permission de l'ayant-droit. Toute action qui serait engagée à la suite d'une utilisation non autorisée d'un élément de l'œuvre sur lequel une tierce partie détient des droits ne pourrait l'être qu'à l'encontre de l'utilisateur.

Ventes, droits et licences. Les produits d'information de la FAO sont disponibles sur le site web de la FAO (www.fao.org/publications) et peuvent être obtenus sur demande adressée par courriel à: publicationssales@fao.org. Les demandes visant un usage commercial doivent être soumises à: www.fao.org/ contact-us/licence-request. Les questions relatives aux droits et aux licences doivent être adressées à: copyright@fao.org. 


\section{Table des matières}

AVANT-PROPOS

REMERCIEMENTS

vii

ABRÉVIATIONS ET ACRONYMES

\section{INTRODUCTION}

\section{APERCU DU PROCESSUS DU PLAN NATIONAL} D'ADAPTATION (PNA)

2.1 Principes directeurs

2.2 Cadre intégrateur pour les plans nationaux d'adaptation et les objectifs de développement durable: l'iFrame des PNA-ODD 7

2.3 Relations entre les PNA, les CND, les autres documents programmatiques et les politiques et mesures nationales et infranationales

2.4 Aperçu des processus des PNA dans les pays

3 POURQUOI ET COMMENT PRENDRE EN COMPTE LES FORÊTS, LES ARBRES ET L'AGROFORESTERIE DANS LES PNA?

3.1 Forêts et autres systèmes avec arbres

3.2 Services écosystémiques fournis par les forêts et les arbres

3.3 Impacts du changement climatique sur les forêts et les arbres 24

3.4 Relations entre les forêts et les systèmes vulnérables

26

4 DISPOSITIONS INSTITUTIONNELLES POUR PRENDRE EN COMPTE LES FORÊTS ET LES ARBRES DANS LES PNA

4.1 Mise en place d'un processus d'adaptation pour les forêts et les arbres

4.2 Assurer la liaison avec les autres sous-secteurs de l'agriculture

4.3 Organiser la participation du secteur forestier la planification de l'adaptation nationale, notamment en clarifiant les mandats et en faisant participer les parties prenantes

\section{PRÉPARER LE TERRAIN ET COMBLER} LES LACUNES

5.1 Faire le point sur les connaissances et les politiques existantes dans le secteur forestier et identifier les lacunes 35

5.2 Évaluer les besoins en capacités et développer les capacités pour la planification de l'adaptation des forêts et des arbres $\mathbf{3 7}$

5.3 Évaluer et identifier les liens entre les besoins d'adaptation et les objectifs de développement

\section{ELÉMENTS DE PRÉPARATION}

6.1 Analyser les scénarios climatiques actuels et futurs en ce qui concerne les forêts et les personnes qui en dépendent $\mathbf{4 5}$

6.2 Identifier et évaluer les vulnérabilités des forêts, des arbres et des personnes qui dépendent des forêts au changement climatique

6.3 Identifier les domaines dans lesquels les forêts et les arbres peuvent contribuer à renforcer l'adaptation au changement climatique dans les secteurs vulnérables identifiés

6.4 Identifier les options d'adaptation pour les forêts, les arbres et les personnes qui dépendent des forêts

6.5 Identifier les moyens de rendre l'environnement plus favorable

6.6 Compiler et communiquer les perspectives sur les forêts et les arbres

6.7 Examiner l'intégration de l'adaptation au changement climatique dans les stratégies, programmes et plans forestiers nationaux et infranationaux

\section{STRATÉGIES DE MISE EN GUVRE}

71

7.1 Accorder aux forêts et aux arbres la priorité requise dans le PNA

7.2 Mettre en place les moyens de permettre un changement transformationnel

7.3 Mobiliser des ressources financières $\quad 74$

7.4 Améliorer la capacité de planification et de mise en œuvre de l'adaptation pour les forêts et les systèmes arboricoles $\mathbf{7 5}$

7.5 Promouvoir la coordination et la synergie aux niveaux national et infranational 


\section{RAPPORTS, SUIVI ET ÉVALUATION}

8.1 Préparer le suivi de la planification et de la mise en œuvre de l'adaptation

8.2 Faire le suivi de la mise en œuvre et mettre à jour le PNA

8.3 Sensibilisation au processus et rapport sur les progrès et l'efficacité

\section{CONCLUSION}

ANNEXES

Annexe 1. Glossaire

\section{5}

Annexe 2. Liste de contrôle des éléments de l'inventaire $\quad \mathbf{8 8}$

Annexe 3. Outils de connaissances

91

Annexe 4. Sous-étapes et méthodologies pour hiérarchiser les options d'adaptation

RÉFÉRENCES

\section{Encadrés, tableaux et figures}

\section{ENCADRÉS}

1. Le PNA de I'Ouganda pour l'agriculture

2. Dialogues sur l'adaptation pour l'identification des risques climatiques et des actions à mener dans le cadre du processus PNA-Ag de I'Uruguay

3. Définitions utilisées pour les évaluations des ressources forestières de la FAO

4. Exemples de questions pour évaluer la capacité institutionnelle du secteur forestier à planifier l'adaptation au changement climatique

5. Évaluation des besoins en capacités de l'autorité nationale de gestion de la sécheresse du Kenya dans le cadre du.PNA

6. Extrait des contributions déterminées au niveau national de l'Indonésie

7. Les personnes qui dépendent de la forêt

8. Un processus de PNA pour l'agriculture sensible au genre en Ouganda

9. La foresterie dans le PNA de l'agriculture de l'Ouganda

10. Évaluation de la viabilité financière et économique des options d'adaptation liées à l'ag roforesterie pour les agriculteurs kenyans

11. Établir des liens entre les Plans nationaux d'adaptation (PNA) et les contributions déterminées au niveau national (CDN) pour améliorer la planification de l'adaptation pou le secteur de l'agriculture au Viet Nam

12. Le développement d'une politique agroforestière au Népal 72

13. Intégration des approches paysagères dans le processus de planification de l'adaptation aux Philippines

14. L'évaluation des ressources forestières mondiales (FRA)
15. Évaluation de l'adaptation des secteurs par étapes en Finlande $\mathbf{8 1}$

16. Exemples de critères génériques importants pour évaluer les options d'adaptation possibles

\section{TABLEAUX}

1. Correspondance entre les directives techniques du PNA et les directives complémentaires

2. Exemples de mesures visant à accroître la résilience des forêts face aux divers impacts du changement climatique $\mathbf{6 0}$

3. Résumé des caractéristiques des principales méthodes d'aide à la décision (économique) pour le choix des mesures d'adaptation au climat

4. Un exemple de tableau synthétique des mesures d'adaptation prioritaires selon leurs bénéfices et leurs coûts

\section{FIGURES}

1. Exemple de processus de formulation et de mise en oeuvre d'un PNA

2. Courbe de transition entre la forêt et l'utilisation des terres $\mathbf{2 1}$

3. Contributions potentielles des forêts, des arbres et de l'agroforesterie à l'adaptation d'autres secteurs/systèmes $\mathbf{2 7}$

4. Flux possibles pour l'intégration des secteurs agricoles dans les PNA (à adapter)

5. Les gestionnaires des forêts réagissent à un large éventail de facteurs

6. Arbre de décision (simplifié) des méthodes potentielles pour évaluer les coûts et les bénéfices des options d'adaptation $\mathbf{1 1 5}$ 


\section{Avant-propos}

\section{INTÉGRATION DE LA FORESTERIE ET DE L'AGROFORESTERIE DANS LES PLANS D'ADAPTATION NATIONAUX}

[ Directives complémentaires ]

Partout dans le monde, les forêts et les arbres ont déjà fait les frais du changement climatique. Les changements de température et de précipitations, les tempêtes, les invasions d'insectes ravageurs et le risque croissant d'incendies plus fréquents et plus intenses menacent les forêts et les moyens d'existence des 1,6 milliard de personnes qui en dépendent (FAO, 2018a).

Il ne fait plus aucun doute que nous avons un énorme défi à relever, non seulement pour contenir le réchauffement climatique, mais aussi pour faire face aux conséquences que nous avons déjà vues et à celles qui sont à venir. La pandémie de la covid-19 a montré les vulnérabilités de nos sociétés et de nos systèmes alimentaires, ce qui met en évidence la nécessité de renforcer la résilience afin d'être prêt à faire face à de nouveaux risques.

Comme le montre cette publication, les forêts, les arbres et l'agroforesterie servent d'interface entre les ressources naturelles et les activités humaines et fournissent ainsi de nombreuses solutions d'adaptation basées sur la nature. C'est pour cela que l'adaptation occupera un rôle majeur dans l'histoire du changement climatique et des forêts. Les forêts doivent s'adapter et devenir plus résilientes afin de pouvoir continuer à fournir les services écosystémiques, y compris ceux dont dépendent d'autres secteurs agricoles tels que les cultures, l'élevage et la pêche. Par exemple, en aidant à prévenir les inondations et l'érosion, les forêts offrent toute une série d'avantages, notamment aux zones urbaines et aux villes, et elles contribuent à l'adaptation des infrastructures, des économies et des populations.
Toutefois, pour réaliser leur potentiel d'adaptation, les forêts et les arbres ont besoin de la stabilité des zones forestières et du régime foncier, ainsi que d'investissements à long terme.

En 2010, la communauté internationale avait mis en place le processus des plans d'adaptation nationaux (PNA) pour les pays les moins avancés (PMA) et pour d'autres pays en développement au titre du cadre de Cancun pour l'adaptation, afin d'identifier et de répondre à leurs besoins d'adaptation à moyen et long terme. L'importance de l'adaptation a par la suite été de plus en plus reconnue. Pas moins de 93 pour cent des pays en développement ont inclus l'adaptation des secteurs agricoles dans leurs contributions déterminées au niveau national (CDN) conformément à l'accord de Paris, tandis que 28 pour cent se réfèrent aux PNA agricoles en particulier (FAO, 2017a). En fait, les PNA sont considérés comme un instrument essentiel pour réaliser les priorités d'adaptation ainsi que les CDN des pays. Comme le processus des PNA concerne l'ensemble de l'économie, il offre la possibilité d'examiner les interactions entre tous les secteurs et leurs implications sur la planification et la mise en œuvre, de manière coordonnée et cohérente. Il offre des possibilités considérables pour une approche plus holistique de l'utilisation des terres et des paysages, ce qui est nécessaire pour une adaptation efficace.

En 2013, le groupe d'experts des pays les moins avancés (LEG) de la Convention-cadre des Nations Unies sur les changements climatiques (CCNUCC) a invité les participants internationaux à élaborer des directives sectorielles supplémentaires pour compléter les directives techniques génériques du 
LEG relatives au processus des PNA. En réponse à cette invitation, l'Organisation des Nations unies pour l'alimentation et l'agriculture (FAO) a lancé en 2017 la publication intitulée Aborder l'agriculture, les forêts et la pêche dans les plans nationaux d'adaptation-Directives supplémentaires.

Lors de sa dernière réunion en 2018, le Comité des forêts de la FAO (COFO) a encouragé les pays à «intégrer les forêts dans leurs plans nationaux d'adaptation, à élaborer des politiques d'adaptation basées sur les forêts, à prendre des mesures pour améliorer la santé des forêts et à restaurer les forêts et les paysages dégradés»».

La FAO et le programme de recherche sur les forêts, les arbres et l'agroforesterie (FTA) du CGIAR se sont associés pour élaborer ces directives complémentaires portant spécifiquement sur les forêts, les arbres et l'agroforesterie et visant à informer:

- Les planificateurs et les décideurs nationaux travaillant sur le changement climatique dans les pays en développement pour leur permettre de mieux comprendre les besoins et les possibilités d'adaptation pour les forêts, les arbres et l'agroforesterie.

- Les autorités et les experts des secteurs forestier et agricole qui contribuent déjà à l'adaptation au changement climatique et à la formulation des PNA.

Cette publication peut également être utilisée par les pays pour informer la préparation des projets d'adaptation tels que ceux qui seront financés par le Fonds vert pour le climat (FVC), y compris les projets de préparation et en particulier ceux qui
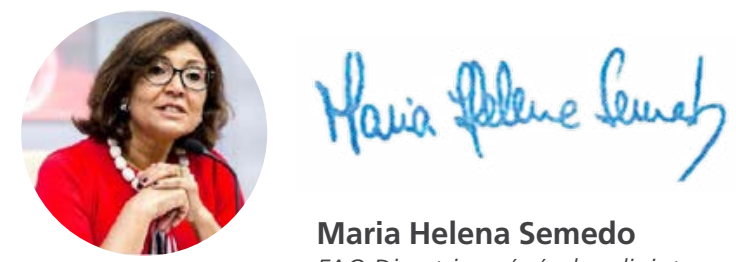

Maria Helena Semedo FAO Directrice générale adjointe entrent dans le cadre du fond de préparation FVCPNA.

Ces nouvelles directives ont vu le jour à l'issue d'un processus consultatif auquel ont participé des experts de pays, d'agences internationales et d'organismes de recherche, ainsi que le secrétariat de la CCNUCC et le LEG. Les directives s'appuient sur les enseignements tirés dans les pays et de ceux issus du programme «Intégrer l'agriculture dans les plans d'adaptation nationaux» (PNA-Ag), programme codirigé par la FAO et le Programme des Nations Unies pour le développement (PNUD). Ce programme a pour objectif de répondre aux besoins d'adaptation au changement climatique dans le secteur agricole en intégrant la question de l'adaptation dans les processus nationaux de planification et de budgétisation des 11 pays partenaires.

Dans le cadre de l'adoption de l'Agenda 2030 des objectifs de développement durable et de l'accord de Paris, la communauté internationale s'est fixée des objectifs collectifs ambitieux. L'utilisation des terres est la pierre angulaire de toutes ces ambitions, en particulier pour ce qui est des engagements pris par les pays et qui sont énoncés dans leurs CDN. En raison de leur rôle important dans l'atténuation, l'adaptation, la gestion durable des ressources naturelles et la sécurité alimentaire, les forêts et les arbres sont au cœur de cette approche intégrée. Nous espérons que cette publication aidera les pays et les autres parties prenantes à intégrer et à hiérarchiser ces objectifs dans le cadre de l'adaptation au changement climatique et des autres processus de planification pertinents.
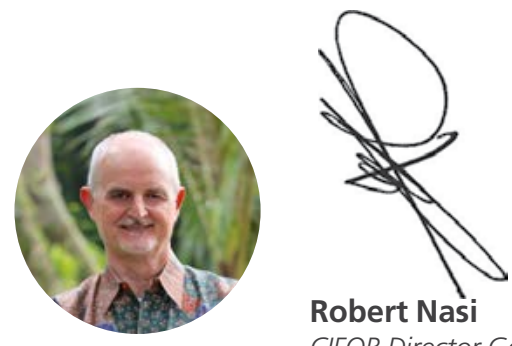

Robert Nasi

CIFOR Director General 


\section{Remerciements}

Les auteurs de l'ouvrage «Addressing forestry and agroforestry in National Adaptation Plans»(Intégration de la foresterie et de l'agroforesterie dans les plans d'adaptation nationaux) sont Alexandre Meybeck et Vincent Gitz du Programme de recherche sur les forêts, les arbres et l'agroforesterie (FTA) du CGIAR qui est dirigé par le Centre pour la recherche forestière internationale (CIFOR), et Julia Wolf et Theresa Wong de l'Organisation des Nations Unies pour l'alimentation et l'agriculture (FAO). Cette publication a été préparée et rédigée en collaboration avec la FTA et le département des forêts et le bureau du changement climatique, de la biodiversité et de l'environnement (OCB).

Les auteurs tiennent à remercier les experts de la FAO dont les noms suivent pour leurs contributions: Simone Borelli, Michela Conigliaro, Krystal Crumpler, Jose Diaz, Elisa Distefano, Claudia Garcia, Cecilia Jones, Paul Maina Guthiga, Willy Kakuru, Daniela Kalikoski, Constance Miller, Peter Moore, Sibyl Nelson, Shanali Pethiyagada, Simmone Rose, Shiroma Sathyapala, Andrew Taber et Victoire De Wever.

Les auteurs tiennent également à remercier les chercheurs du FTA dont les noms suivent: Christopher Martius (CIFOR), Meine van Noordwijk (ICRAF), Marlene Elias (Bioversity), Houria Djoudi (CIFOR), Roeland Kindt (ICRAF), Beria Leimona
(ICRAF), Lasisa A. Duguma (ICRAF), Bruno Locatelli (CIRAD/CIFOR), Daniel Murdiyarso (CIFOR), Yves Laumonier (CIRAD/CIFOR), Catherine Muthuri (ICRAF), Eduardo Somarriba (CATIE), Bimbika Sijaparti Basnett (CIFOR), Aster Gebrekirstos (ICRAF), Chris Kettle (Bioversity), Thanglong Trinh (INBAR), Herman Savenije (Tropenbos), Peter Minang (ICRAF), Fergus Sinclair (ICRAF), Ramni Jamnadass (ICRAF) et Pablo Pacheco (CIFOR).

Enfin, les auteurs tiennent à exprimer leur gratitude aux experts qui ont fait des contributions lors des consultations organisées par le GIZ le 2 mai 2018 à Bonn, en Allemagne et lors du PNA Expo 2019 qui s'est tenu à Incheon, en Corée.

La version précédente de cette publication a bénéficié des conseils et de l'examen par les pairs des experts suivants: Ali Raza Rizvi, Suzan Braatz, Janie Rioux et Michael Huettner. Nous leur sommes très reconnaissants du temps précieux qu'ils y ont consacré. Le contenu final reste de la seule responsabilité des auteurs.

Nous tenons également à exprimer notre gratitude à Rebecka Ramstedt pour ses conseils sur les communications concernant les directives et pour les travaux connexes, ainsi qu'à Art\&Design pour la conception et la mise en page. 


\section{Abréviations et acronymes}

$\mathrm{AbE}$

AND

CBD

CCNUCC

CDN

COP

CPDN

DRM

DDR

FAO

FEM

FVC

GES

GIEC

LEG

MAAIF

MARD

MGAP

NDMA

ODD

ONG
Adaptation basée sur les écosystèmes

Autorité désignée au niveau national

Convention sur la diversité biologique

Convention-cadre des Nations Unies sur les changements climatiques

Contribution déterminée au niveau national

Conference of the Parties (Conférence des Parties)

Contribution prévue déterminée au niveau national

Disaster risk management (Gestion des risques de catastrophes)

Disaster risk reduction (Réduction des risques de catastrophes)

Organisation des Nations Unies pour l'alimentation et l'agriculture

Fonds pour l'environnement mondial

Fonds vert pour le climat

Gaz à effet de serre

Groupe d'experts intergouvernemental sur l'évolution du climat

Groupe d'experts des pays les moins avancés

Ministère de l'agriculture, de l'industrie animale et de la pêche

Ministère de l'agriculture et du développement rural

Ministère de l'élevage, de l'agriculture et de la pêche

Autorité nationale de gestion de la sécheresse

Objectifs de développement durable

Organisation non gouvernementale 
OSC

OSCST

PANA

PEID

pfn

PMA

PNA

PNUD

REDD+

SFM

UICN

UNCCD
Organisations de la société civile

Organe subsidiaire de conseil scientifique et technologique

Programme d'action national aux fins de l'adaptation

Petits États insulaires en développement

programmes forestiers nationaux

Pays les moins avancés

Plan national d'adaptation

Programme des Nations Unies pour le développement

Réduction des émissions dues à la déforestation et à la dégradation des forêts

Société française de microbiologie

Union internationale pour la conservation de la nature

Convention des Nations Unies sur la lutte contre la désertification 


\section{Introduction}

Les discussions sur la question des forêts et des arbres dans le contexte du changement climatique ont longtemps porté principalement sur leur potentiel d'atténuation. Cependant, le potentiel d'atténuation des forêts et des arbres dépend également de leur capacité à s'adapter au changement climatique et à la pression humaine accrue sur les ressources qu'ils fournissent. En outre, les forêts et les arbres jouent un rôle crucial dans la résilience des paysages et des populations ainsi que dans leur capacité à s'adapter au changement climatique. Le processus des plans d'adaptation nationaux (PNA) offre une opportunité de mieux intégrer ces rôles dans les stratégies et les politiques nationales.

La contribution des forêts et des arbres aussi bien à l'adaptation qu'à l'atténuation est associée à, et dépend de, nombreuses politiques sectorielles qui orientent l'utilisation des terres et de l'eau, notamment celles qui concernent l'aménagement du territoire, la gestion de l'eau, l'énergie, le bâtiment et l'agriculture. Puisque le PNA concerne l'ensemble de l'économie, il donne l'occasion d'examiner les interactions entre tous les secteurs économiques de manière coordonnée et cohérente. En fait, pour arriver á une adaptation efficace, il est nécessaire d'intégrer ces interactions et d'en tirer des enseignements pour la planification et la mise en œuvre. L'objectif de cette publication est de faciliter l'intégration des forêts, des arbres et de l'agroforesterie dans un tel processus.

Cette publication intitulée Intégration de la foresterie et de l'agroforesterie dans les plans d'adaptation nationaux: directives complémentaires fournit des conseils techniques sur l'intégration des forêts, de l'agroforesterie et des arbres dans la formulation et la mise en œuvre des PNA. Elle vise à informer les décideurs politiques et les responsables gouvernementaux chargés de la planification et des processus des PNA, les acteurs du secteur forestier et les autres acteurs intéressés sur les besoins d'adaptation de la foresterie et de l'agroforesterie et sur leur potentiel à soutenir l'adaptation d'autres secteurs, soussecteurs et activités.

PUERTO MALDONADO, MADRE DE DIOS, PÉROU

Traitement des noix du Brésil.

๑) CIFOR/Marco Simola 
Les présentes directives complémentaires visent donc à:

- démontrer la nécessité de l'adaptation des forêts et des arbres;

- démontrer l'importance des forêts et des arbres pour l'adaptation;

- démontrer la nécessité d'intégrer de manière appropriée les forêts et les arbres dans le processus du PNA;

- aider les praticiens des PNA à intégrer la gestion des forêts et des arbres dans les PNA;

- soutenir les acteurs des secteurs forestier et agricole dans leur engagement et leur contribution au processus, au niveau national, à l'échelle de l'économie, et en même temps au niveau local et dans leur secteur/chaîne de valeur;

- déclencher et faciliter un dialogue politique intersectoriel tourné vers l'avenir qui intègre pleinement les forêts et les arbres dans la planification de l'adaptation de tous les secteurs.

Cette publication a été conçue pour les organisations et les individus impliqués dans la planification et les activités d'adaptation nationales, à la fois du secteur forestier et d'autres secteurs qui peuvent bénéficier des forêts et des arbres pour leur adaptation, ou qui ont une influence sur leur capacité d'adaptation.

Cette publication complète deux autres documents clés relatifs à la planification de l'adaptation nationale: premièrement, les directives techniques sur les PNA préparées par le groupe d'experts des pays les moins avancés (PMA) de la Conventioncadre des Nations Unies sur les changements climatiques (CCNUCC, 2012), et deuxièmement, les orientations intersectorielles intitulées Addressing agriculture, forestry and fisheries in National Adaptation Plans - Supplementary guidelines (Prendre en compte l'agriculture, la Foresterie et la pêche dans les plans d'adaptation nationaux - Directives supplémentaires) préparées par la FAO (appelées ici «directives PNA-Ag») (Karttunen et al., 2017). Elle sera complétée par le document à venir intitulé Addressing Fisheries and Aquaculture in National Adaptation Plans (FAO, 2020a).
Elle mobilise l'ensemble des connaissances existantes en matière de gestion des forêts, d'évaluation de la vulnérabilité et d'adaptation au changement climatique, en s'appuyant sur l'expérience de la FAO et du programme de recherche sur les forêts, les arbres et l'agroforesterie du CGIAR (FTA). Afin de répondre efficacement aux besoins des pays, la publication s'appuie également sur une analyse des PNA déjà publiés et des documents comparables préparés par les pays développés ou les autorités infranationales. La publication a bénéficié en particulier du programme PNA-Ag et des recommandations du LEG ainsi que des consultations impliquant des experts techniques et des acteurs clés des organisations de la société civile, des organisations non gouvernementales, du secteur privé et des organisations internationales.

Compte tenu des multiples liens des forêts, des arbres et de l'agroforesterie avec d'autres activités et secteurs, des contributions qu'ils apportent à leur adaptation ainsi qu'aux multiples objectifs de développement durable (ODD), cette publication adopte une approche systémique suivant le cadre intégrateur pour les plans nationaux d'adaptation et les objectifs de développement durable (NAP-SDG iFrame) récemment défini par le LEG. Une telle approche permet d'intégrer différentes approches d'évaluation dans les PNA et d'envisager de manière plus explicite la façon d'aborder les ODD par le biais des PNA.

Cette publication définit le contexte en fournissant un aperçu du processus des PNA (chapitres 1 et 2) et en soulignant l'importance d'intégrer les forêts et les arbres dans la formulation et la mise en œuvre des PNA (chapitre 3). Elle fournit ensuite des indications sur les forêts, les arbres et les activités agroforestières dans les PNA, organisées selon les quatre éléments des directives techniques de la CCNUCC pour les PNA (voir tableau 1): (i) élément A, préparer le terrain et combler les lacunes (chapitres 4 et 5); (ii) élément B, préparation (chapitre 6); (iii) élément $\mathrm{C}$, stratégies de mise en œuvre (chapitre 7); et (iv) élément D, rapports, suivi et évaluation (chapitre 8). 
TABLEAU 1.

Correspondance entre les directives techniques du PNA et les directives complémentaires

\begin{tabular}{|l|c|l|}
\hline Élément A. Préparer le terrain et combler les lacunes & $\rightarrow$ & Chapitres 4 et 5 \\
\hline Élément B. Préparation & $\rightarrow$ & Chapitre 6 \\
\hline Élément C. Stratégies de mise en œuvre & $\rightarrow$ & Chapitre 7 \\
\hline Élément D. Rapports, suivi et valuation & $\rightarrow$ & Chapitre 8 \\
\hline
\end{tabular}

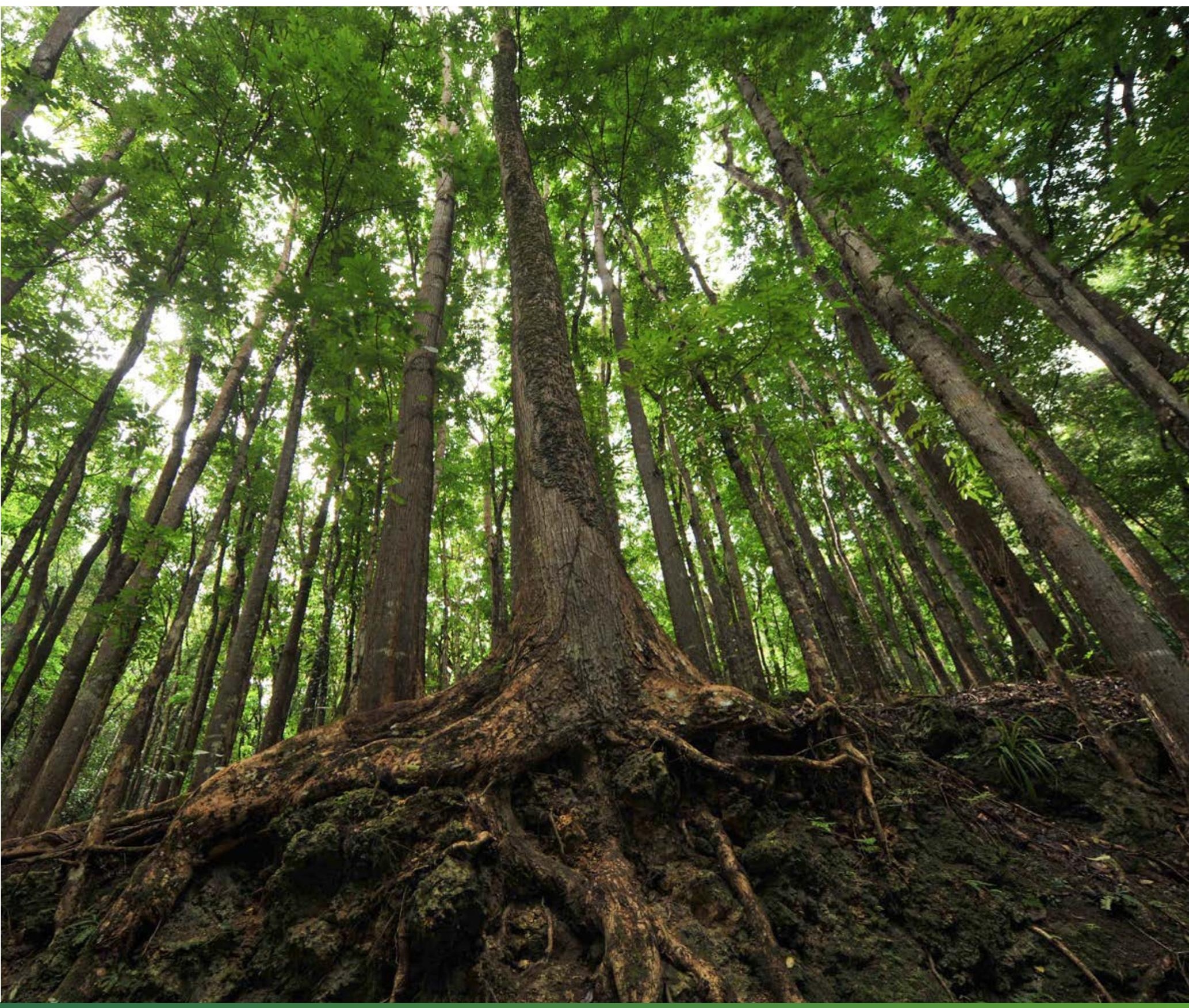




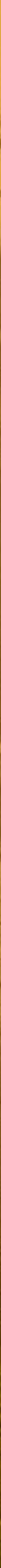




\section{- Aperçu du processus du plan national d'adaptation (PNA)}

\author{
L'objectif de \\ ce chapitre est \\ d'inscrire la prise en \\ compte des forêts \\ et des arbres dans \\ la perspective $d u$ \\ processus des PNA \\ tel que défini par \\ la CCNUCC et mis \\ en ouvre par les \\ pays au travers \\ de processus et \\ d'orientations \\ nationales \\ spécifiques.
}

\subsection{Principes directeurs}

Le processus des PNA a été établi dans le cadre de la CCNUCC, en tant qu'élément du cadre de Cancún pour l'adaptation adopté en 2010 lors de la seizième session de la Conférence des parties à la CCNUCC (COP 16). L'objectif du processus des PNA est de permettre aux pays les moins avancés (PMA) et aux autres pays en développement d'identifier les besoins d'adaptation à moyen et long terme et d'élaborer et de mettre en œuvre des stratégies et des programmes pour répondre à ces besoins (décision 1/CP.16).

Les objectifs du processus des PNA, convenus lors de la COP 17 (décision 5/CP.17), sont les suivants: i) réduire la vulnérabilité aux effets du changement climatique en renforçant la capacité d'adaptation et la résilience; et ii) faciliter une intégration cohérente de l'adaptation au changement climatique, aux politiques, programmes et activités pertinents, nouveaux et existants, en particulier aux processus et stratégies de planification du développement, dans tous les secteurs concernés et à différents niveaux, le cas échéant.

La décision 5/CP.17 énumère les principes directeurs du processus d'élaboration des PNA, à savoir:

- un processus de planification continu au niveau national avec des mises à jour et des résultats itératifs;

- qui est la propriété du pays, qui est dirigé par le pays;

- qui n'est pas normatif, mais flexible et basé sur les besoins des pays;

- qui s'appuie sur les efforts d'adaptation existants et évite de les dupliquer;

- qui est participatif et transparent;

- qui renforce la cohérence de la planification de l'adaptation et du développement;

- qui est soutenu par une surveillance et un examen complet;

- qui tient compte des groupes, des communautés et des écosystèmes vulnérables; 
- qui est guidé par les meilleures données scientifiques disponibles;

qui prend en compte les connaissances traditionnelles et indigènes;

qui est sensible au genre.

Les premières directives pour la formulation des PNA figurent dans l'annexe de la décision 5/CP.17. Elles sont complétées par les directives techniques pour le processus d'élaboration des $P N$, qui ont été élaborées par le Groupe d'experts des pays les moins avancés en réponse au paragraphe 15 de la décision 5/CP.17 et sont disponibles en plusieurs langues ${ }^{1}$. Ces directives techniques constituent labase de la formulation et de la mise en œuvre des PNA (voir figure 1).
Des suppléments aux directives ont été élaborés pour divers secteurs et sont disponibles sur le site du NAP central ${ }^{2}$. Les suppléments sur les secteurs agricoles préparés par la FAO dans le cadre du programme PNA-Ag, ainsi que les directives pour soutenir l'intégration de la diversité génétique dans la planification de l'adaptation au changement climatique et dans les PNA présentent un intérêt particulier pour le secteur forestier. De même, les directives sur la biodiversité (CDB, 2014) et celles qui sont relatives á l'eau (PRP, 2019) sont particulièrement pertinentes.

\section{FIGURE 1.}

\section{Exemple de processus de formulation et de mise en œuvre d'un PNA}

Elément A:

Jeter les bases et combler les lacunes

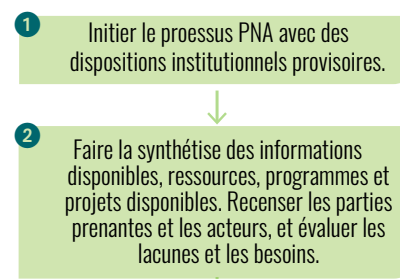

État des lieux; analyse des lacunes et rapport sur les besoins; cartographie et profil des parties.

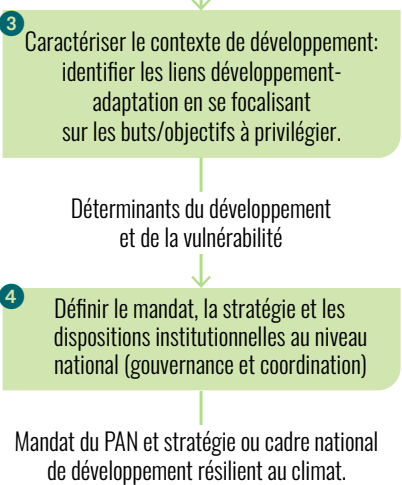
de développement résilient au climat.
Base de données des options d'adaptation

9 Identifier les options d'adaptation qui prennent en compte les principales vulnérabilités et les activités visant à intégrer l'adaptation dans la planification

Rapport d'évaluation de la vulnérabilité et des risques climatiques

Évaluation de la vulnérabilité et des risques climatiques

Rapport d'analyse des risques et scénarios

7 Analyser les scénarios climatiques passé et futurs et caractériser le risque climatique.

Rapport sur les perspectives d'avenir

Elément B: 6 Eléments préparatoires

$$
\begin{aligned}
& \text { Envisager des scénarios futurs et } \\
& \text { des actions de développement et } \\
& \text { d'adaptation à un climat changeant. }
\end{aligned}
$$

Évaluation, hiérarchisation et classement des options d'adaptation

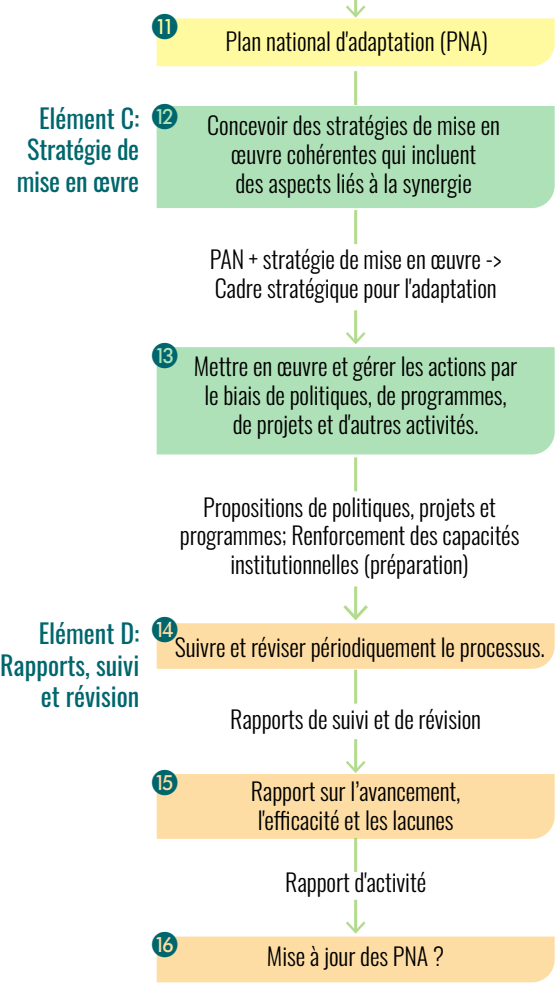

1 Accessible ici: https://www4.unfccc.int/sites/NAPC/Guidelines/Pages/Technical-guidelines.aspx

2 Accessible ici: https://www4.unfccc.int/sites/NAPC/Guidelines/Pages/Supplements.aspx 


\subsection{Cadre intégrateur pour les plans nationaux d'adaptation et les objectifs de développement durable: l'iFrame des PNA-ODD}

L'article 7 de l'accord de Paris prévoit un objectif global en matière d'adaptation qui est le suivant: améliorer la capacité d'adaptation, renforcer la résilience et réduire la vulnérabilité au changement climatique en vue de contribuer au développement durable et de garantir une réponse d'adaptation adéquate dans le cadre de l'objectif de limitation de l'augmentation moyenne de la température mondiale à moins de $2^{\circ} \mathrm{C}$.

Par conséquent, l'accord intègre sa contribution au développement durable dans le cadre de l'objectif d'adaptation. La plupart des pays qui ont intégré une composante d'adaptation dans leur contribution nationale déterminée (CND)³, ont également défini un objectif ou une vision à long terme pour l'orienter (CCNUCC, 2015). Ces objectifs sont étroitement liés aux objectifs de développement tels que l'éradication de la pauvreté, le développement économique ou l'amélioration du niveau de vie, de la sécurité et des droits de l'homme; et dans certains cas, ils mentionnent explicitement les objectifs de développement durable (ODD). Plusieurs pays en développement ont mentionné l'objectif d'être un pays émergent avec une économie à revenu moyen d'ici 2030. Par exemple, la CND de l'Éthiopie combine des objectifs ambitieux de développement, d'atténuation et d'adaptation en donnant une place centrale aux secteurs de l'agriculture, y compris la protection et le rétablissement des forêts pour leurs services économiques et écosystémiques afin d'arriver à plus de 7 millions d'hectares boisés ou reboisés.

Ces objectifs et buts de développement sont susceptibles d'orienter la préparation des PNA correspondants. En fait, comme le PNA est un vaste processus national orienté vers l'avenir, il doit tenir compte des objectifs nationaux à moyen et long terme. Contrairement aux programme d'action national aux fins de l'adaptation (PANA) qui répondent aux besoins les plus urgents des PMA, les PNA s'inscrivent sur le long terme et doivent donc prendre en considération l'adaptation d'un pays qui est lui-même en évolution.

Pour ces raisons, et également compte tenu des liens intrinsèques entre l'adaptation et la réalisation des objectifs de développement durable, le groupe d'experts invite les pays à adopter un cadre intégrateur pour les plans d'adaptation nationaux et les objectifs de développement durable, l'iFrame PNA-ODD, afin de faciliter l'intégration des approches et l'examen explicite de la manière de contribuer à la réalisation des objectifs de développement durable par le biais des PNA (CCNUCC, 2017).

Le LEG note que les approches systémiques intégratives sont utiles pour aborder la cohérence et la synergie des mesures d'adaptation à de multiples échelles et niveaux, y compris dans le temps, en tenant compte d'autres cadres pertinents tels que les ODD et le Cadre de Sendai pour la réduction des risques de catastrophe 20152030 (CCNUCC, 2018). Une approche systémique de l'adaptation peut grandement faciliter et renforcer l'intégration de la foresterie dans les PNA, en tirant parti de son potentiel de changement transformationnel. Les forêts et autres formations arborées doivent elles-mêmes être appréhendées comme des systèmes.

Les forêts et les arbres sont étroitement liés et souvent intégrés à d'autres systèmes agricoles. Par les services écosystémiques qu'elles fournissent, les forêts sont également liées à plusieurs autres grands systèmes: biodiversité, eau, production d'énergie, établissements humains, pour reprendre les thèmes identifiés par les pays eux-mêmes (voir section 3.4).

Compte tenu des relations profondes qui existent entre les forêts, les arbres et les autres systèmes, l'adoption d'une approche systémique de l'adaptation permet une prise en compte plus efficace des contributions des forêts et

3 Les CDN incarnent les efforts de chaque pays pour réduire les émissions nationales et s'adapter aux impacts du changement climatique. L'Accord de Paris (article 4, paragraphe 2) exige que chaque Partie prépare, communique et maintienne les CDN successives qu'elle entend réaliser. 
des arbres. En outre, elle facilite l'articulation des différentes échelles où différents secteurs/ systèmes interagissent, en particulier les paysages et même les ménages. Enfin, elle permet aux pays de prendre en compte de manière appropriée l'environnement institutionnel, économique et social qui détermine ces différentes relations. Ceci est particulièrement important pour identifier les vulnérabilités sociales, les leviers/contraintes au changement ainsi que les résultats potentiels des mesures d'adaptation et la répartition des coûts et des bénéfices.

Une telle approche systémique intégrée facilite également l'intégration de l'adaptation dans la planification de divers objectifs et engagements concernant les forêts et la restauration des terres, comme par exemple ceux qui découlent des ODD, de l'accord de Paris, du plan stratégique 2017-2030 des Nations Unies pour les forêts et du défi de Bonn sur la restauration des paysages forestiers.

\subsection{Relations entre les PNA, les CND, les autres documents programmatiques et les politiques et mesures nationales et infranationales}

Comme indiqué précédemment, l'un des objectifs du processus des PNA est de faciliter une intégration cohérente de l'adaptation au changement climatique dans les politiques, programmes et activités pertinents, nouveaux et existants, en particulier dans les processus et stratégies de planification du développement, dans tous les secteurs concernés et à différents niveaux, le cas échéant. En fait, comme l'indique l'iFrame du PNA-ODD, la meilleure façon d'atteindre cet objectif est d'adopter une approche systémique intégrée des secteurs et des acteurs, ainsi que de l'adaptation.

Le PNA est le moyen d'aligner l'adaptation sur d'autres objectifs et mesures, en particulier ceux qui sont liés aux ODD, aux objectifs d'atténuation du changement climatique, ainsi qu'aux grandes stratégies nationales de développement. Les pays ont exprimé leur engagement à aborder le changement climatique dans leur CDN. La mise en œuvre exigera que les pays modifient les politiques et mesures nationales existantes ou en adoptent de nouvelles. Dans de nombreux cas, les pays ont explicitement mentionné la réalisation d'un PNA dans leur CDN - le PNA peut être un véhicule important pour la mise en œuvre des engagements d'adaptation (voir l'exemple du Viet nam dans l'encadré 11). De nombreux pays ont également pris dans leur CDN un engagement d'atténuation par la foresterie et, dans certains cas, ils ont explicitement fait le lien entre l'atténuation et l'adaptation pour la foresterie. D'autres engagements ont été pris dans les CDN pour la restauration des terres dégradées. Tous ces engagements peuvent également contribuer à la réalisation d'autres objectifs mondiaux.

Puisque que le PNA vise à modifier ou à créer des politiques et des mesures, souvent à différents niveaux de gouvernement et en impliquant divers secteurs et catégories d'acteurs, l'efficacité de son processus dépend en fait de sa capacité à influencer et à modifier d'autres processus. Il est donc particulièrement important de savoir comment le processus peut faciliter la coordination au sein des secteurs et entre les secteurs et les politiques concernées, ainsi que la coordination entre les différents niveaux de gouvernement et entre les acteurs, tant à l'intérieur du secteur qu'avec ceux qui lui sont liés.

\subsection{Aperçu des processus des PNA dans les pays}

La plupart des pays en développement sont en train de développer leur PNA; en fait, 20 d'entre eux ont déjà transmis un $\mathrm{PNA}^{4}$ au secrétariat de la CCNUCC. En outre, de nombreux pays développés ont élaboré des plans d'adaptation. Ces documents donnent un premier aperçu des différentes approches adoptées par les pays, notamment la manière dont les PNA peuvent être liés à d'autres stratégies, la manière dont ils sont élaborés et organisés par secteurs ou régions, et la manière dont la foresterie est représentée.

4 Consulté au 2 juin 2020. Disponible sur le portail du PNA, périodiquement mis à jour: https://www4.unfccc.int/sites/NAPC/Pages/national-adaptation-plans.aspx 
Les PNA font souvent référence, de manière explicite, à d'autres documents nationaux préexistants sur le changement climatique ainsi qu'aux stratégies et plans de développement. Dans de nombreux cas, et notamment pour les PNA qui ont été parmi les premiers à être achevés, le processus s'est appuyé sur des stratégies et des plans antérieurs en matière de changement climatique. Dans de tels cas, le PNA peut faire partie d'une stratégie ou d'un plan plus vaste en matière de changement climatique. Par exemple, le Sri Lanka disposait déjà, au moment de la finalisation de son PNA, d'une politique nationale sur le changement climatique adoptée en 2012, et d'une stratégie nationale d'adaptation au changement climatique (2011-2016). Le pays a ainsi précisé que le PNA est un plan évolutif comportant un ensemble d'actions réalisables. Il est axé sur des interventions pratiques; les stratégies et les principes étant déjà énoncés dans les documents précédents.

Plusieurs autres pays ont, explicitement ou implicitement, formulé des principes et des stratégies plus vastes, qui peuvent figurer déjà dans un autre document sur le changement climatique, ainsi que des interventions plus pratiques qui doivent être incluses dans le PNA. Une telle focalisation sur les actions pratiques peut également être liée aux possibilités attendues de sources internationales spécifiques de financement supplémentaire.

Dans leur PNA, les pays distinguent généralement les besoins et les activités transversales de ceux

qui sont sectoriels. Cette distinction peut conduire à l'élaboration de deux sections distinctes: une section générale (ou un document) contenant des principes, des questions et activités transversales et des sections sectorielles (ou des documents), qui constituent souvent l'essentiel du PNA. Dans certains pays, comme au Brésil et au Chili, ces sections constituent des documents distincts. Dans d'autres cas, comme en Palestine et au Soudan, le PNA adopte une structure infranationale (régionale). Par exemple, le PNA du Soudan part d'une analyse des vulnérabilités et propose des mesures d'adaptation menées au niveau de l'État (infranational), l'objectif explicite étant d'avoir comme axe principal du plan une meilleure compréhension des défis d'adaptation au niveau de l'État. Certains pays, comme la Colombie, adoptent les deux approches: un plan national et des plans régionaux en cours de préparation. En outre, la plupart des PNA intègrent la possibilité d'avoir des actions qui sont sous-nationales, en particulier sur des sujets où la compétence a été dévolue à un niveau sous-national de gouvernement (Kenya par exemple). Certains plans d'adaptation nationaux sont organisés de manière à servir de cadre et de toile de fond aux plans infranationaux (Australie, Canada par exemple).

Lorsque le PNA est organisé par secteurs (voir exemple dans l'encadré 1), ces secteurs sont généralement choisis en raison de leur vulnérabilité particulière au changement climatique et/ou de leur rôle dans l'adaptation globale du pays. Les secteurs et leur délimitation précise sont généralement adaptés pour correspondre à des ministères de tutelle spécifiques afin de faciliter la préparation et la mise en œuvre du plan.

ENCADRÉ 1.

\section{Le PNA de l'Ouganda pour l'agriculture}

Le Plan national d'adaptation du secteur agricole en Ouganda a été lancé en novembre 2018 et constitue le plan du pays pour l'intégration des priorités et des préoccupations du secteur agricole dans le PNA ougandais global (MAAIF, 2018). Le plan a été lancé en janvier 2015 par le groupe de travail sur le changement climatique au sein du Ministère de l'agriculture, de l'industrie animale et de la pêche (MAAIF), en coordination avec le Ministère de l'eau et de l'environnement et avec le Ministère des finances, de la planification et du développement économique. Le soutien technique et financier est venu du projet de la FAO «Alliance mondiale contre le changement climatique» (FAO et UE) et du programme PNA-Ag. 
Le plan contient 21 options d'adaptation chiffrées et hiérarchisées dans les domaines suivants: gestion de la pêche; information sur le climat; alerte précoce et préparation aux catastrophes; foresterie, gestion des terres et des ressources naturelles; recherche et gestion des connaissances. Une analyse coût-bénéfice a été utilisée pour la priorisation des différentes actions.

Le PNA ougandais pour l'agriculture a été formulé dans le respect des directives techniques du PNA. Tout d'abord, un projet de cadre d'action pour le secteur agricole a été élaboré, suivi de lignes directrices destinées aux planificateurs nationaux et provinciaux pour l'intégration du changement climatique dans les plans du secteur agricole. Un effort a été fait pour tirer les leçons de l'expérience du PANA de l'Ouganda (2007) ainsi que de la vision globale de la politique nationale en matière de changement climatique. Les principales étapes ont été les suivantes:

- un bilan pour identifier les informations disponibles sur les impacts et la vulnérabilité au climat afin de déterminer les points d'entrée pour l'adaptation au changement climatique dans le secteur agricole;

- une formation sur les outils d'évaluation des options d'adaptation pour les parlementaires, les groupes de jeunes, les planificateurs au niveau central et au niveau des districts, et les acteurs non étatiques afin de renforcer les capacités d'évaluation des options d'adaptation, l'analyse de genre et l'intégration du genre dans les plans et la mise en œuvre de l'adaptation;

- des consultations avec les parties prenantes pour recueillir des informations sur les impacts économiques et sociaux actuels et attendus du changement climatique.

\section{Principales caractéristiques du plan}

1. Consultation et inclusion - Dès le début, la priorité a été donnée à un processus participatif et inclusif. Des consultations ont été menées auprès de fonctionnaires nationaux et locaux de différents secteurs tels que l'agriculture, l'environnement, l'eau, l'égalité des sexes et la jeunesse, et avec le secteur privé, les partenaires au développement et des représentants de la société civile. Des participants de six districts du corridor central pour le bétail (Sembabule, Mubende, Kiboga, Luwero, Nakasongola et Nakaseke) ont également été inclus. Les activités suivantes ont été menées:

- des consultations avec les collectivités locales afin d'identifier les priorités du secteur agricole à prendre en compte dans le PNA, de recueillir des expériences sur la mise en œuvre des PANA et de recenser les bonnes pratiques;

- des visites de projets pilotes PANA ayant permis de tirer des enseignements de la mise en œuvre des technologies d'adaptation et des projets PANA lancés en 2007 pour alimenter le processus des PANA;

- des ateliers consultatifs (trois jours) impliquant les collectivités locales et les participants du corridor du bétail pour partager les impacts et les interventions prioritaires existantes, les approches et les partenaires potentiels.

2. Vers un PNA tenant compte de la dimension de genre - Le genre étant l'un des huit thèmes prioritaires, une stratégie de développement des capacités en matière de genre a été formulée par le biais d'une évaluation détaillée des 
besoins, de formations adaptées et de visites sur le terrain afin que les décideurs puissent mieux comprendre les défis auxquels sont confrontés les agriculteurs et les agricultrices (voir encadré 8).

\section{Une approche par zone agroécologique permettant de tirer les leçons de}

l'expérience. Tirant les leçons des projets d'adaptation passés, le plan a adopté l'approche consistant à définir des mesures d'adaptation pour les différentes zones agroécologiques. Cela permettra de guider les agriculteurs, les agents techniques et les décideurs sur les investissements qui sont conscients des impacts en amont et en aval des actions.

\section{Prochaines étapes}

L'étape suivante est la mise en œuvre du plan. Les projets en cours et futurs devraient aligner leurs activités sur les recommandations. De plus, de nouvelles modalités de coordination ont été recommandées. Le PNA pour l'agriculture propose que le groupe de travail sur le changement climatique de la MAAIF soit officiellement reconnu en tant que groupe de travail fonctionnel. Une unité de coordination de l'agriculture sur le changement climatique (ACCU) sera créée pour préciser les fonctions, mettant en œuvre à la fois le PNA et le programme national d'agriculture intelligente face au climat.

Le plan sera mis à jour tous les cinq ans en cohérence avec le cycle de mise à jour du Plan national de développement, tandis que le cycle de planification de la MAAIF intégrera certaines des actions clés. Il est prévu de soumettre au Parlement des directives sur l'intégration de la dimension de genre dans l'adaptation au changement climatique dans les plans, budgets et politiques au niveau local. Ces directives incluront des guides pour différents sous-secteurs. Des efforts sont en cours pour mobiliser des sources de financement nationales et internationales afin de mettre en œuvre le plan et d'en diffuser les résultats. Le PNA pour l'agriculture est le premier PNA sectoriel en Ouganda et les leçons tirées de son élaboration et de sa mise en œuvre sont intégrées dans le processus global du PNA par le biais d'ateliers d'engagement et d'intégration dans les projets.

Source: FAO et PNUD. 2020a. Étude de cas sur l'intégration de l'agriculture dans les plans nationaux d'adaptation - Ouganda, Rome.

Les forêts sont souvent incluses dans le secteur biodiversité/écosystèmes du PNA, généralement parce que dans de nombreux pays, le Ministère de l'environnement est responsable de la foresterie. Par exemple, dans le PNA kenyan, l'une des mesures proposées pour le secteur de l'environnement est de préparer un plan d'adaptation pour les forêts; une autre est de renforcer les initiatives de plantation d'arbres et de conservation. Au Chili, par exemple, les forêts sont incluses dans le plan de biodiversité du PNA, mais les forêts plantées font partie du plan sylvopastoral. Dans la plupart des PNA publiés, l'agroforesterie est couverte dans la section agriculture des PNA.
Les pays ont mis en place des dispositions institutionnelles spécifiques pour préparer et mettre en œuvre leur PNA. Ces arrangements s'appuient généralement sur les institutions existantes, avec l'ajout d'organes et de mécanismes de coordination spécifiques au niveau central. Dans certains cas, ils sont complétés par des mécanismes de coordination infranationaux.

Conformément à l'objectif d'intégration de l'adaptation au changement climatique dans les politiques et les budgets, les pays s'appuient généralement sur les ministères et les organismes existants pour gérer l'adaptation de leurs secteurs respectifs, plutôt que sur des institutions 
spécialisées mandatées pour le changement climatique dans son ensemble. Par exemple, le PNA du Cameroun décrit en détail les rôles et responsabilités des différents ministères, du Parlement, des différents niveaux de l'administration nationale, des autorités locales, ainsi que du secteur privé, de la société civile, des autorités traditionnelles et religieuses, des médias, de la recherche et des universités dans la mise en œuvre du PNA.

Pour la plupart des PNA qui étaient disponibles au moment de l'élaboration de cette publication, la coordination générale de la préparation et de la mise en œuvre est assurée par la combinaison d'un ministère de tutelle et d'un organe collégial qui rassemble des représentants de différents ministères et agences sous l'autorité d'un ministre, souvent le Ministère de l'environnement ou, dans certains cas, du Premier ministre ou du chef de l'État. Dans de nombreux cas, c'est l'organe collégial qui détient l'autorité générale. Dans d'autres cas, il s'agit du ministère de tutelle, comme au Sri Lanka, avec le Secrétariat du changement climatique au sein du Ministère du développement et de l'environnement du Mahaweli, l'organe collégial ayant principalement pour rôle de superviser les questions transversales. Le principal ministère de tutelle est généralement celui qui est chargé des politiques relatives au changement climatique, souvent le Ministère de l'environnement, mais pas toujours. En Finlande, le Ministère de l'agriculture et des forêts est chargé de coordonner la mise en œuvre du plan national d'adaptation au changement climatique et les différents ministères sont responsables de la mise en œuvre, du suivi et de l'évaluation du plan au sein de leurs branches administratives respectives. Souvent, les organisations de coordination sont complétées par un organe consultatif, qui peut être un groupe d'experts ou un conseil comprenant des représentants de la société civile et du secteur privé.

Les mécanismes de coordination des PNA s'appuient souvent sur des mécanismes de coordination globale préexistants en matière de changement climatique. C'est par exemple le cas pour le Sri Lanka avec le rôle de coordination du Secrétariat du changement climatique, y compris pour les plans sectoriels. C'est également le cas au Chili, où c'est le Conseil des ministres de la durabilité et du changement climatique qui approuve le PNA, les plans sectoriels et leurs modifications. Au Cameroun, la responsabilité globale de la préparation et de la mise en œuvre du PNA est exercée par le comité de pilotage du processus national de réduction des émissions dues à la déforestation et à la dégradation des forêts (REDD+), avec utilisation des structures existantes. Ces structures peuvent faciliter l'intégration des synergies et des compromis entre l'atténuation et l'adaptation qui sont particulièrement importants pour les secteurs de l'agriculture et surtout de la foresterie.

Les PNA ne donnent souvent pas beaucoup de détails sur la façon dont les différents secteurs sont organisés. La responsabilité semble généralement être confiée au ministère responsable du secteur. Dans certains cas, comme au Sri Lanka, un rôle défini est également attribué explicitement à chaque entité sectorielle par l'entité directrice générale.

Certains PNA mentionnent explicitement des formes de coordination et d'organisation au niveau infranational, soit sous la responsabilité des niveaux infranationaux de l'administration nationale, soit déléguées aux autorités locales, selon l'organisation institutionnelle du pays. Ils peuvent également mentionner des formes explicites de coordination entre les niveaux régional et national; par exemple, au Chili, un point focal régional sur le changement climatique du Ministère de l'environnement est chargé de la liaison avec le département du changement climatique du même ministère, tandis qu'au Cameroun, les structures de coordination régionales et provinciales pour l'adaptation rendent compte au comité de pilotage national.

Bien que la responsabilité de la préparation et de la mise en œuvre du PNA incombe au gouvernement, il existe diverses formes d'implication des acteurs non gouvernementaux, en particulier la société civile et le secteur privé. Au niveau central, il existe souvent un organe consultatif composé de représentants de la société civile et du secteur privé. Il existe également des cas de conseils consultatifs régionaux, comme au Chili. Le Brésil a organisé une consultation publique sur un premier projet de plan et a pris en considération les commentaires formulés pour le finaliser. En Uruguay, le processus du PNA a inclus des dialogues avec les parties prenantes pour identifier les risques et les vulnérabilités (voir encadré 2). Dans son examen des progrès réalisés, le secrétariat de la CCNUCC (2018) note que même si les formes diffèrent d'un pays à 
l'autre, il existe des preuves de l'engagement de la société civile et d'autres parties prenantes tout au long du processus de formulation et de mise en œuvre des PNA. Il existe également des preuves de l'utilisation des connaissances et de l'expérience des communautés locales. Une étude menée par Southern Voices on Adaptation (2018) au Cambodge, au Kenya, au Malawi, au Népal, au Sri Lanka et au Viet nam a mis en évidence les progrès réalisés en matière de participation et de transparence. Les participants à l'étude, tout en reconnaissant qu'il reste de nombreux défis à relever, ont noté l'impact positif que la décentralisation du processus peut avoir sur la participation et la transparence.

\title{
ENCADRÉ 2
}

\section{Dialogues sur l'adaptation pour l'identification des risques et des actions à mener dans le cadre du processus PNA-Ag de l'Uruguay}

\begin{abstract}
En Uruguay, le Plan national d'adaptation à la variabilité et au changement climatique pour le secteur agricole (PNA -Agro), lancé en septembre 2019, est un instrument stratégique qui aidera le pays à réaliser les engagements nationaux d'adaptation et d'atténuation établis dans son premier PNA dans le cadre de l'Accord de Paris, et contribuera aux ODD (MGAP, 2019). L'élaboration du PNAAgro a impliqué un processus de consultation des parties prenantes sur trois ans au sein et au-delà des secteurs agricoles (MGAP, 2019). Connues sous le nom de «Dialogues sur l'adaptation», ces consultations ont permis d'identifier les priorités nationales en matière d'adaptation, les défis et les réponses à apporter pour alimenter le Plan. Ce processus a été mené par le Bureau des programmes et politiques agricoles du Ministère de l'élevage, de l'agriculture et de la pêche, en coordination avec le Système national de réponse au changement et à la variabilité climatiques. Un soutien technique a été fourni par le PNUD et la FAO, dans le cadre du programme PNA-Ag.
\end{abstract}

Ces dialogues, auxquels ont participé plus de 200 personnes, ont permis d'identifier les impacts de la variabilité et du changement climatique ainsi que les stratégies d'adaptation prioritaires axées sur les différents systèmes de production, les femmes rurales et l'agriculture familiale. Les dialogues ont porté sur la Foresterie, la production de riz, l'agriculture familiale, le système laitier et le genre.

Le dialogue sur l'adaptation du système laitier a été le premier dialogue à avoir lieu. Les participants ont identifié trois principaux défis climatiques au niveau des exploitations: l'excès de précipitations, le déficit en eau et la sécheresse, et le stress thermique. Le dialogue a permis de constater qu'il y avait un manque d'outils et d'infrastructures pour adapter avec succès le système de production laitière au changement et à la variabilité climatiques. En outre, il a montré qu'il y avait un consensus sur le fait que la production laitière devait s'adapter à la variabilité accrue du climat et au changement climatique, et que le système devait être basé sur le pâturage direct dans les lieux de pâturages et avec des réserves stratégiques de fourrage produit à la ferme et utilisé comme complément alimentaire, avec un minimum d'intrants extérieurs à l'exploitation. Ce système nécessite des infrastructures pour que les bovins puissent se rafraîchir et se mettre à l'ombre pendant l'été; en outre, des routes et des surfaces sont nécessaires pour canaliser les précipitations excessives et faciliter le déplacement du bétail et des récoltes. 
Le dialogue a également souligné l'importance des aspects sociaux et économiques de la production laitière. Dans l'ensemble, les participants ont estimé qu'il était nécessaire de développer des systèmes de production qui attirent les jeunes générations dans les exploitations. Cela va des systèmes automatisés qui prévoient du temps pour le repos et les loisirs, à la nécessité de mettre en place des instruments financiers, une assurance contre les risques climatiques et des fonds pour aider à atténuer l'effet des fluctuations de prix et de l'augmentation des coûts de production liés aux événements climatiques extrêmes.

Le dialogue sur l'adaptation des forêts a été organisé avec le soutien de l'Association uruguayenne des producteurs forestiers (Sociedad de productores forestales). Le dialogue a analysé les risques et les vulnérabilités associés à différents événements climatiques, tels que l'augmentation des conditions de sécheresse entraînant une hausse des températures qui peut se traduire par une augmentation des maladies et des parasites des plantes, une perte de production et un risque accru d'incendie. Un autre risque important qui avait été identifié était l'excès d'eau qui est dû à des pluies abondantes et qui peut entraîner une combinaison d'effets d'entraînement économiques et de production. Les pertes de production ont également été identifiées comme étant le résultat potentiel de problèmes phytosanitaires, de l'érosion des sols et de difficultés logistiques lors de la récolte qui peuvent entraîner une perte de qualité du bois. Il a été convenu que le réchauffement climatique et les projections relatives à l'intensité accrue des phénomènes météorologiques en Uruguay entraîneraient une augmentation des facteurs de risque mentionnés.

Un dernier dialogue sur l'adaptation s'est concentré sur des sujets transversaux et a vu la participation des agriculteurs familiaux de tous les systèmes de production. Le Dialogue sur l'adaptation de l'agriculture familiale a été organisé en collaboration avec la section nationale de la Réunion spécialisée des agriculteurs familiaux (REAF) du Marché commun du Sud (MERCOSUR). Plus de quarante agriculteurs, dont des producteurs de cultures, des producteurs laitiers, des éleveurs de bétail, des pêcheurs, des éleveurs d'ovins, ainsi que des producteurs de fruits et des horticulteurs ont fait part de leurs préoccupations et ont contribué au dialogue qui a permis d'identifier les réponses d'adaptation à privilégier dans le PNA-Agro.

On peut tirer de ces exemples quelques conclusions générales concernant l'intégration de la foresterie et de l'agroforesterie dans le processus du PNA. Dans la plupart des pays, l'élaboration et la mise en œuvre du PNA sont organisées par secteurs, généralement en cohérence avec les ministères concernés, avec l'objectif explicite de faciliter l'intégration de l'adaptation. La coordination générale est assurée par la combinaison d'une administration chef de file et d'un organe interministériel de coordination qui joue également un rôle de premier plan dans la conception et la mise en œuvre des activités transversales. Une telle organisation, tout en étant une première étape indispensable et propice à l'intégration de l'adaptation dans les secteurs, pourrait ne pas être en mesure de faciliter la coopération intersectorielle sur les mesures d'adaptation. En fait, la Finlande a conçu une graduation de l'adaptation en cinq étapes et inclut la coopération intersectorielle dans les critères du plus haut niveau d'adaptation (voir encadré 14). Ceci est particulièrement important pour les forêts et les arbres, étant donné leur contribution potentielle à l'adaptation d'autres secteurs et l'influence d'autres secteurs sur leur vulnérabilité et leur capacité d'adaptation. La foresterie est souvent intégrée dans le secteur des écosystèmes/e la biodiversité, mais cela peut nuire à la reconnaissance méritée de sa contribution à des objectifs autres que ceux de la biodiversité, comme par exemple l'économie ou les moyens d'existence, en particulier chez les plus vulnérables, les populations autochtones, les communautés locales, 
les femmes et les minorités ethniques. Comme le montrent les exemples de plans qui prévoient une forte composante infranationale (Soudan, Canada, Australie), c'est souvent à ce niveau que les diverses contributions des forêts et des arbres à l'adaptation sont les plus reconnues et renforcées.
Les échanges entre les secteurs au niveau local, ainsi qu'entre les niveaux local et national, sont donc particulièrement importants pour faciliter l'intégration de la foresterie et de l'agroforesterie dans la conception et la mise en œuvre des politiques d'adaptation globales.
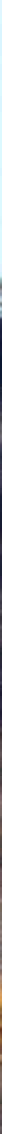
(2.

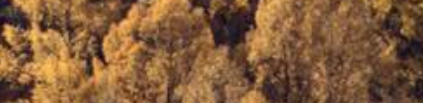
Ext.

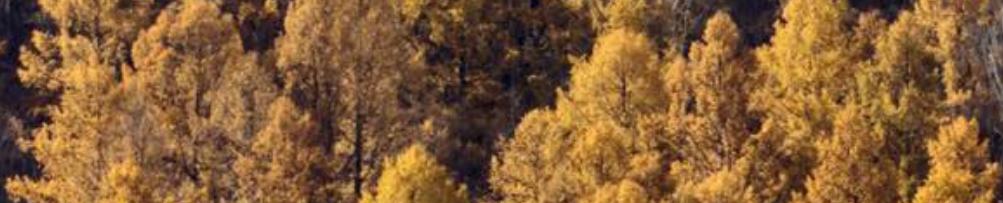

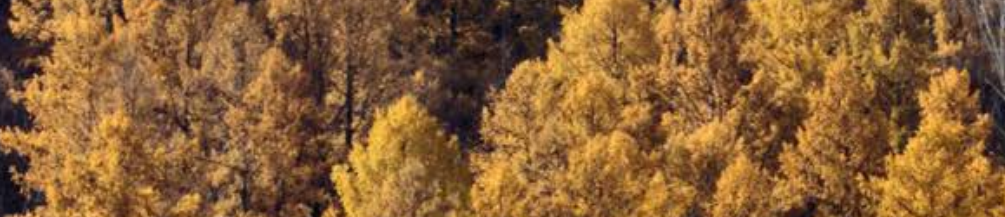

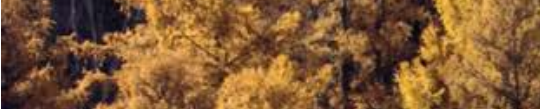
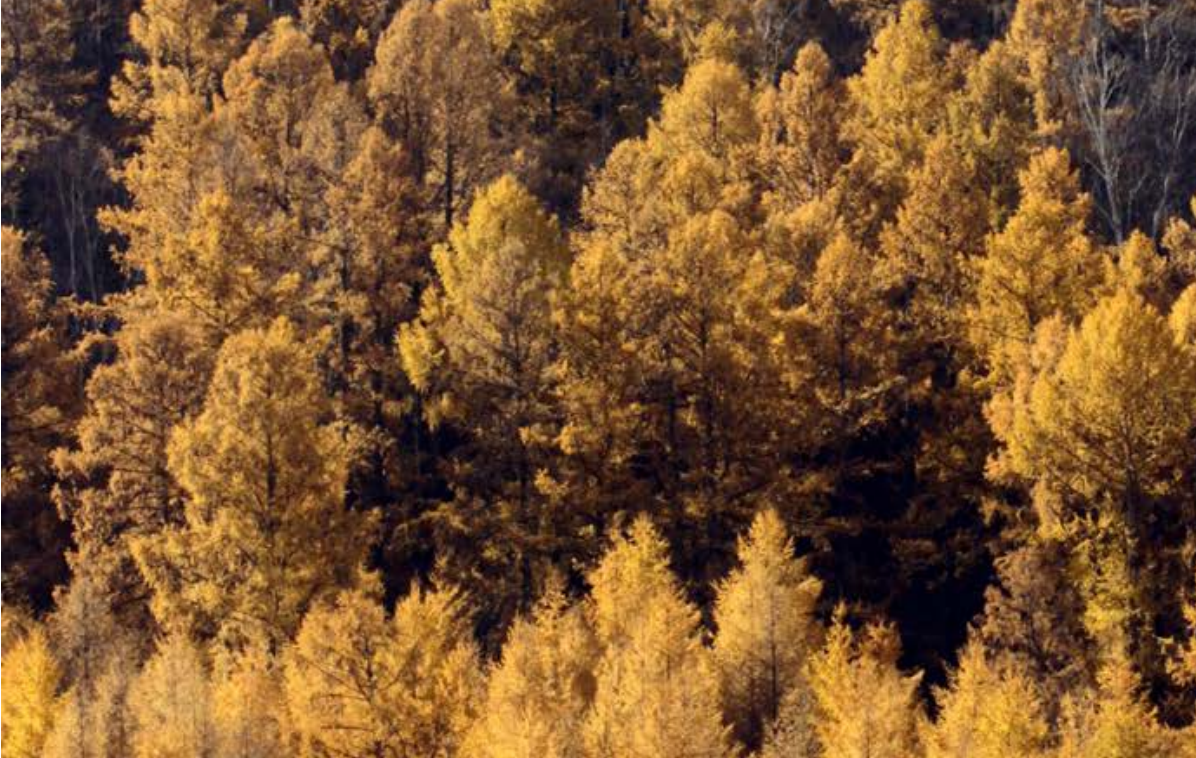

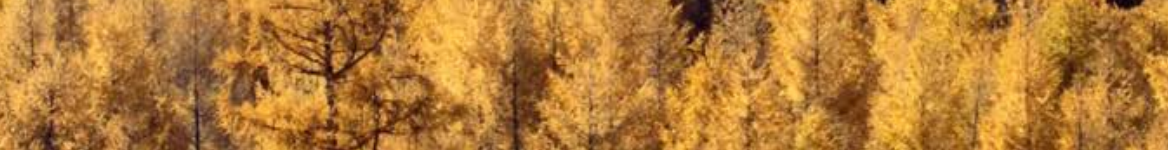
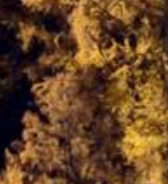

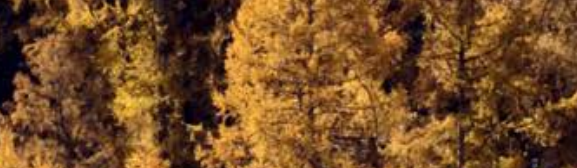

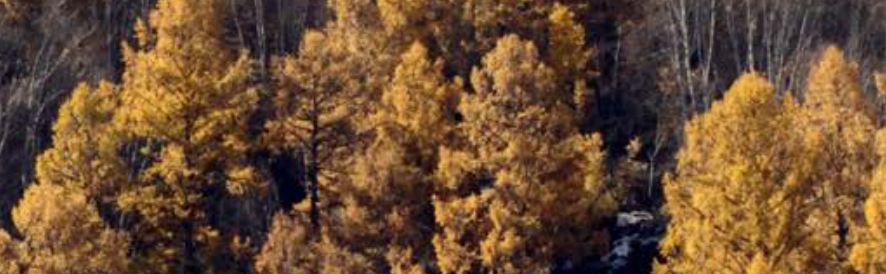
(2) 18.2. -
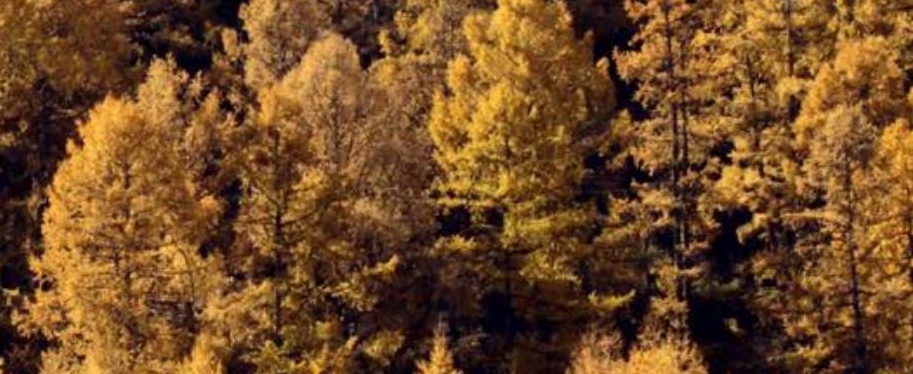


\section{- Pourquoi et comment prendre en compte les forêts, les arbres et l'agroforesterie dans les PNA?}

\author{
Ce chapitre \\ souligne les \\ principales raisons \\ pour lesquelles \\ les forêts et les \\ arbres (y compris \\ l'agroforesterie, \\ les vergers, les \\ arbres hors forêt, \\ les forêts urbaines \\ et périurbaines) \\ doivent être \\ intégrés de manière \\ appropriée dans la \\ formulation et la \\ mise en œuvre des \\ PNA.
}

Les premières raisons communes à tous les autres sous-secteurs agricoles ont trait à leur vulnérabilité particulière au changement climatique. Un deuxième groupe de raisons est basé sur les multiples biens et services écosystémiques fournis par les forêts et les arbres, dont la plupart seront en même temps menacés par le changement climatique. Le troisième groupe de raisons, souvent sous-estimé, est que la foresterie et les biens et services fournis par les forêts et les arbres peuvent être appelés à jouer un rôle essentiel dans l'adaptation de la plupart des autres secteurs, des systèmes identifiés comme vulnérables (tels que les cultures, le bétail, les systèmes d'eau), et des personnes et populations vulnérables. C'est pourquoi les forêts et les arbres sont une composante essentielle des solutions basées sur la nature (AbE) qui sont définies comme des actions qui utilisent les écosystèmes pour aider les sociétés à relever de manière durable une variété de défis environnementaux, sociaux et économiques. Les AbE sont explicitement considérées comme des alternatives aux technologies, à l'ingénierie et aux infrastructures créées par l'homme. Elles intègrent la conservation et la protection de la biodiversité comme objectif de base. Les AbE sont de plus en plus encouragées pour faire face au changement climatique et à d'autres défis sociétaux (MacKinnon et al., 2008 ; IUCN 2009). Le rôle des forêts et des arbres est envisagé et géré au mieux au niveau du paysage, ce qui nécessite une approche paysagère de l'adaptation.

Pour toutes ces raisons, et conformément aux recommandations du LEG, ce chapitre adopte une approche systémique. Il part de la description de la diversité des forêts et des systèmes arborés et de leurs contributions à d'autres secteurs/systèmes à différents niveaux, puis examine leurs principales vulnérabilités au changement climatique et la manière dont ils peuvent contribuer au mieux à l'adaptation de ces autres secteurs/systèmes. 


\subsection{Forêts et autres systèmes avec arbres}

Cette publication traite des forêts selon leur définition de la FAO (voir encadré 3), des zones décrites comme forêts au niveau national même si elles ne correspondent pas strictement à la définition de la FAO, et d'autres systèmes arboricoles comme l'agroforesterie qui sont très souvent soumis au même type d'impacts liés au climat et fournissent très souvent le même type de services écosystémiques. En outre, il examine la dynamique entre les catégories d'utilisation des terres (avec l'agriculture), car elles ont une forte influence sur leur vulnérabilité au changement climatique.

\subsubsection{Une diversité de forêts et une diversité de perspectives sur les forêts}

Les forêts sont extrêmement diverses, selon la latitude, l'altitude, la topographie, la pente, la température, le régime des pluies, la composition du sol, les interactions plantes-animaux et les activités humaines historiques et actuelles. Elles sont également définies de manière très différente, sous de nombreux angles, par différents acteurs: comme un type spécifique d'écosystème; comme une zone produisant du bois pour une entreprise commerciale, comme une zone produisant une diversité de produits ligneux et non ligneux dans une forêt communautaire; comme le territoire traditionnel d'une tribu indigène; comme une zone de chasse; comme un espace de loisirs; comme une zone de conservation; etc. Cette diversité de points de vue sur les «forêts» explique le nombre et la diversité des définitions juridiques utilisées aux niveaux national et international, souvent à des fins différentes.

Au niveau mondial, l'évaluation des ressources forestières (FRA) de la FAO a contribué à l'harmonisation des approches utilisées pour définir et classer les forêts (voir encadré 3). L'évaluation des ressources forestières utilise une définition mondiale des forêts qui comprend un seuil minimum pour la hauteur des arbres (5 $\mathrm{m})$, un couvert forestier d'au moins 10 pour cent et une taille minimale de la zone forestière $(0,5$ ha). Les parcs urbains, les vergers, les plantations de palmiers à huile, l'agroforesterie et les autres cultures d'arbres agricoles sont exclus de la définition de la FAO (mais le caoutchouc, le chêne-liège et les plantations d'arbres de Noël sont inclus).

\section{ENGADRÉ 3}

\section{Définitions utilisées pour les évaluations des ressources forestières de la FAO}

\section{Forêt}

«Terrain de plus de 0,5 hectare avec des arbres de plus de 5 mètres de haut et un couvert végétal de plus de 10 pour cent, ou avec des arbres capables d'atteindre ces seuils in situ. Elle n'inclut pas les terres qui sont principalement utilisées à des fins agricoles ou urbaines».

Parmi les forêts, la FAO distingue à nouveau trois catégories:

- La forêt primaire: «Forêt naturellement régénérée d'espèces indigènes, où il n'y a pas d'indications clairement visibles d'activités humaines et où les processus écologiques ne sont pas sensiblement perturbés».

- Les autres forêts naturellement régénérées: «Forêt naturellement régénérée où il $\mathrm{y}$ a des indications clairement visibles d'activités humaines».

- Les forêts plantées: «Forêts composées principalement d'arbres établis par plantation et/ou ensemencement délibéré». Cette catégorie comprend les «plantations», définies comme des «Forêts plantées qui sont gérées de manière intensive et qui répondent à TOUS les critères suivants au moment de la plantation et de la maturité des arbres: une ou deux espèces, une classe d'âge homogène et un espacement régulier. Sont spécifiquement exclues: les forêts plantées à des fins de protection ou de restauration de l'écosystème». 


\section{Autres terres boisées}

«Terrain non défini comme «forêt», d'une superficie supérieure à 0,5 hectare, avec des arbres de plus de 5 mètres de haut et un couvert végétal de 5 à 10 pour cent, ou avec des arbres capables d'atteindre ces seuils, ou encore avec un couvert combiné d'arbustes, de buissons et d'arbres supérieur à 10 pour cent. Elles n'incluent pas les terres qui sont principalement utilisées à des fins agricoles ou urbaines».

\section{Autres terres avec couvert végétal}

«Terres considérées comme «Autres terres», qui sont principalement utilisées à des fins agricoles ou urbaines et qui présentent des parcelles de plus de 0,5 hectare avec un couvert végétal de plus de 10 pour cent d'arbres pouvant atteindre une hauteur de 5 mètres à maturité. Elle comprend des espèces d'arbres forestières et non forestières»》.

Cette sous-catégorie comprend les groupes d'arbres et les arbres dispersés (par exemple les «arbres hors forêt») dans les paysages agricoles et les zones urbaines, en respectant les trois critères décrits ci-dessus. Elle comprend en particulier les plantations d'arbres fruitiers et les systèmes agroforestiers, ainsi que les plantations d'arbres établies principalement à des fins autres que la production de bois, comme par exemple les plantations de palmiers à huile.

* Plus de détails/explications sur les définitions peuvent être trouvés dans FAO (2018c, 2018d).

\subsubsection{Autres systèmes arborés}

Il existe de nombreux systèmes arborés qui ne sont pas couverts par la définition des forêts de la FAO, soit à cause d'une couverture de canopée plus faible, soit à cause de leur taille plus petite, soit à cause de leur classification comme zone agricole ou urbaine. Parmi ces systèmes figure la catégorie «autres terres boisées» qui comprend les zones boisées qui ne sont pas considérées comme des forêts par la FAO parce que, même si elles répondent aux critères de taille et de hauteur, elles ne répondent pas aux critères de couverture de la canopée (voir encadré 3). Certains pays ont une définition des forêts plus large que celle de la FAO et une partie de ces systèmes relèvent de la définition nationale de la forêt. Il existe également une grande variété de systèmes qui sont étroitement liés aux activités agricoles, tels que les différents systèmes agroforestiers (y compris les systèmes sylvopastoraux), les paysages en mosaïque où les parcelles de forêt sont trop petites pour être considérées comme des forêts à des fins statistiques, ainsi que les plantations d'arbres agricoles comme les palmiers à huile, les oliviers et les vergers (arbres à fruits et à noix).

L'agroforesterie est le nom collectif qui est donné aux systèmes et technologies d'utilisation des terres où les plantes vivaces ligneuses (arbres, arbustes, palmiers, bambous, etc.) sont délibérément utilisées dans les mêmes unités de gestion des terres que les cultures agricoles et/ou les animaux, dans une certaine forme d'arrangement spatial ou de séquence temporelle. Dans les systèmes agroforestiers, il existe des interactions à la fois écologiques et économiques entre les différentes composantes (Lundgren et Raintree, 1982). En d'autres termes, les arbres font partie intégrante du système, qu'ils jouent un rôle de service prédominant (par exemple, les brisevent) ou qu'ils fournissent l'un des principaux produits commerciaux (par exemple, les cultures intercalaires avec les cultures de plantation). Une conception et une gestion appropriées des systèmes agroforestiers peuvent en faire des puits de carbone efficaces, avec un rôle potentiellement important pour l'atténuation. Puisqu'elle fournit des produits et des services qui, autrement, proviendraient des forêts, l'agroforesterie est également une stratégie très précieuse pour améliorer les moyens d'existence locaux tout en réduisant la pression sur les forêts naturelles. La présence d'arbres dans les paysages agricoles contribue également à réduire la vulnérabilité et à accroître la capacité d'adaptation au changement climatique grâce à leurs contributions biophysiques et économiques. 
Les paysages en mosaïque avec des arbres et des fragments de forêt fournissent divers services écosystémiques, notamment des services de régulation du cycle de l'eau, de pollinisation et de lutte contre les parasites (Ricketts, 2004; Ricketts et al., 2008; Holzschuch et al., 2010) et, selon leur répartition, ils peuvent contribuer à la connectivité des zones forestières, réduisant ainsi l'impact de la fragmentation qui peut affecter la santé de la forêt et induire une perte de biodiversité (Bogaert et al., 2011). En outre, la fragmentation et la faible connectivité des parcelles forestières affectent la capacité des pollinisateurs, des ennemis naturels des parasites, de l'eau et des nutriments à se déplacer dans un paysage (Vira et al., 2015).

Les plantations de cultures arboricoles agricoles (telles que les plantations de palmiers à huile, de café, de cacao ou d'oliviers, ainsi que les vergers) partagent de nombreuses caractéristiques des forêts de plantation (telles que définies dans l'encadré 3), en particulier celles qui couvrent de grandes superficies. Les petits vergers et/ ou les vergers mixtes sont proches des systèmes agroforestiers ou en font partie.

Les systèmes d'arbres et les arbres individuels situés dans les zones urbaines et périurbaines constituent ce que l'on appelle la forêt urbaine d'une ville. Ces systèmes peuvent jouer un rôle en compensant - dans une certaine mesure l'énorme contribution des zones urbaines au changement climatique et, plus important encore, ils peuvent rendre les villes plus résistantes aux impacts climatiques. En fait, une gestion stratégique des forêts et des arbres dans et autour des établissements urbains peut faire baisser les températures locales, réduire la consommation d'énergie pour la climatisation et le chauffage, atténuer les inondations ainsi que l'impact du ruissellement des eaux pluviales en augmentant la surface urbaine perméable, et également augmenter la qualité et la disponibilité de l'eau.

\subsubsection{Dynamique des forêts et autres systèmes d'arbres}

Dans de nombreuses régions du monde, les forêts et les systèmes arboricoles évoluent rapidement, sous l'influence de nombreux facteurs, aux niveaux local et mondial: demandes croissantes de terres et de forêts, abandon de certaines zones agricoles et préoccupations environnementales. Ces facteurs peuvent modifier profondément la vulnérabilité des forêts et des systèmes arborés au changement climatique, notamment si l'on considère les perspectives à moyen terme.

Dans un pays donné, la superficie des forêts et du couvert arboré est souvent soumise à des changements importants avec le temps. Historiquement, avec le temps et avec le développement économique et les transitions démographiques, les pays connaissent généralement une diminution de leur superficie forestière, suivie pour certains d'une augmentation ultérieure, avec des changements à la fois dans le type et la quantité de couverture forestière dans les paysages. Par conséquent, les forêts et les systèmes arborés évoluent au fil du temps le long de ce qui a été décrit comme la courbe de transition des forêts, qui comprend les phases de déforestation et de dégradation des forêts, ainsi que le potentiel de restauration des terres dégradées, ex-forêts, sous forme de forêts productives et/ou d'agroforêts. Il est important de comprendre ces changements réels ou potentiels car ils auront souvent une influence sur la vulnérabilité des forêts et des arbres au changement climatique et à d'autres stress ainsi que sur leur capacité à contribuer au bien-être humain.

La courbe de transition forestière (voir figure 1) illustre comment un continuum d'intensité de gestion, allant de faible à élevé, rythme l'«évolution» de la perte et de la reconstitution des forêts et du couvert végétal (Mather et Needle, 1998). 


\section{FIGURE 2.}

\section{Courbe de transition entre la forêt et l'utilisation des terres}

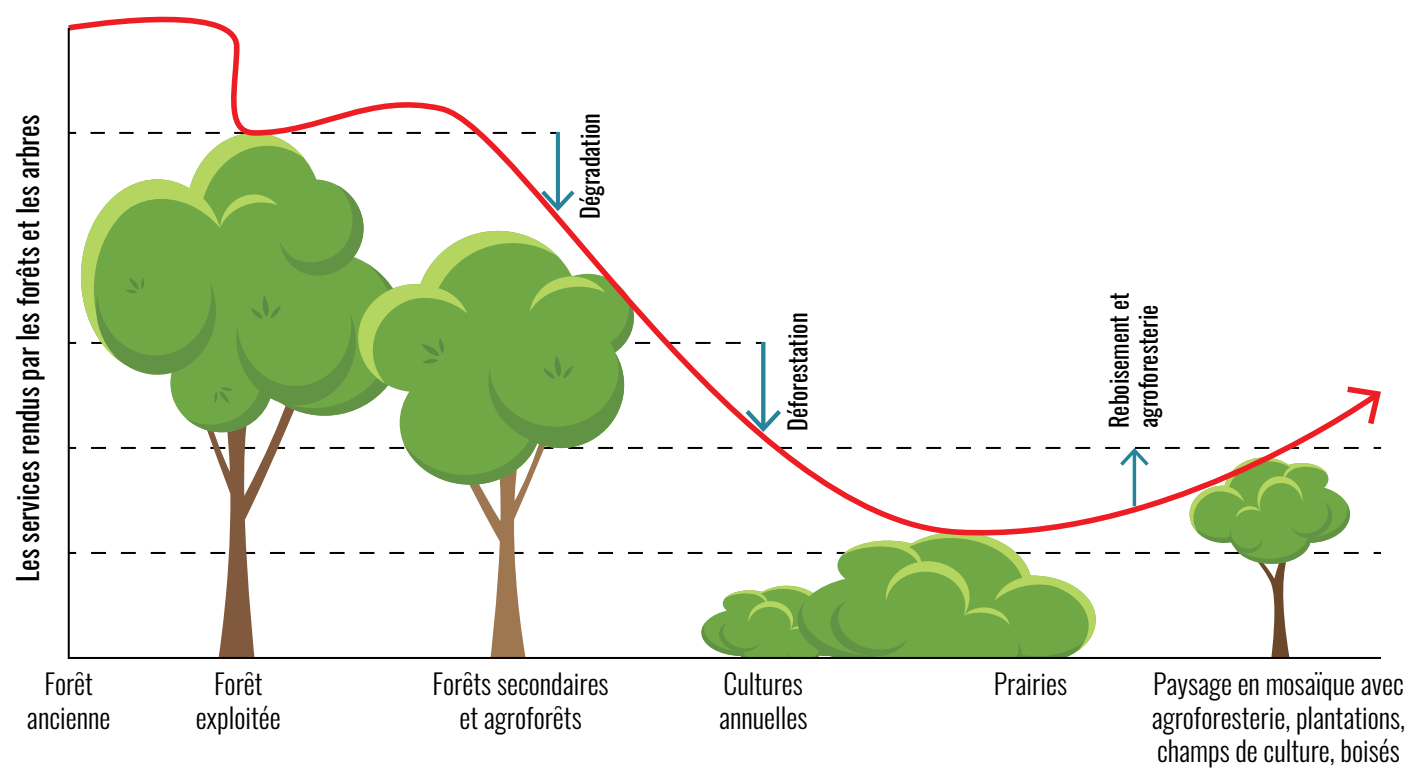

\subsection{Services écosystémiques fournis par les forêts et les arbres}

Les forêts et les arbres fournissent des services essentiels à différents niveaux allant du terrain et des communautés locales aux niveaux national, régional et mondial. Ces services peuvent contribuer à réduire la vulnérabilité de la société au changement climatique (Locatelli et al., 2008; Meybeck, Rose et Gitz, 2019). Les forêts fournissent de la nourriture, de l'énergie, des matériaux et de nombreux autres services écosystémiques dont les populations dépendent à des degrés divers pour leurs moyens d'existence, leur sécurité alimentaire et leur nutrition, notamment par la régulation de l'approvisionnement en eau douce, la conservation de la biodiversité et l'atténuation du changement climatique. Ces contributions sont menacées par le changement climatique, avec des impacts sur les moyens d'existence, la sécurité alimentaire et la nutrition. En outre, les forêts revêtent souvent une importance particulière pour les moyens d'existence en temps de crise, ces derniers étant à leur tour susceptibles de se multiplier et de s'intensifier en raison du changement climatique.
Une grande variété d'aliments d'origine animale et végétale sont consommés par les personnes vivant dans les forêts ou à proximité (Jamnadass et al., 2015; HLPE, 2017) et peuvent représenter une part importante de leur alimentation, en particulier si l'on tient compte de la teneur en nutriments. Par exemple, des études menées au Gabon (Blaney et al., 2009) et en République-Unie de Tanzanie (Powell et al., 2013b) ont estimé la contribution des aliments sauvages à 20 pour cent de l'apport en fer et à plus de 30 pour cent pour la vitamine $\mathrm{A}$, deux des nutriments pour lesquels il existe des carences majeures. En outre, comme le soulignent Vira et al. (2015), les forêts et les systèmes arboricoles diversifiés peuvent fournir des vitamines tout au long de l'année grâce à différentes phénologies de fructification. La viande de brousse, les poissons et les insectes sont une source importante de protéines, en particulier dans certaines régions, tant dans les forêts tropicales, tempérées que boréales (HLPE, 2017). Les forêts et les arbres soutiennent également les systèmes d'élevage en pâturage dans de nombreuses régions.

Le bois de chauffage, qu'il s'agisse de bois ou de charbon de bois, est une source d'énergie importante pour la cuisson et l'ébullition de l'eau, souvent la seule disponible dans les zones rurales des pays en développement. Au niveau mondial, on 
estime que 2,4 milliards de personnes dépendent du bois de chauffage pour cuisiner, dont les deux tiers des ménages africains (FAO, 2017b). Les forêts et les arbres fournissent également de nombreux matériaux utilisés pour la construction, le mobilier, les outils, l'artisanat ainsi que de nombreuses substances utilisées dans la médecine traditionnelle et occidentale.

Ces biens sont également commercialisés, à l'échelle locale, régionale et même mondiale, ce qui détermine deux catégories de bénéficiaires: les consommateurs et tous ceux qui tirent un revenu de la collecte, de la transformation et de la distribution de ces biens. Par exemple, une étude de la FAO (2014) a estimé que 19 pour cent de la population d'Afrique, 15 pour cent de l'Asie et de l'Océanie et 8 pour cent de l'Amérique latine étaient engagés dans la production de bois de chauffage et de charbon de bois en 2011, principalement à temps partiel. Nombre de ces activités sont informelles et ne sont pas entièrement couvertes par les statistiques nationales. De plus, beaucoup d'entre elles sont fortement différenciées selon le sexe, ce qui nécessite une analyse approfondie par sexe. Outre l'emploi et les revenus générés par la collecte et le commerce de marchandises, les forêts génèrent des revenus et des emplois publics et privés dans l'industrie des loisirs et du tourisme, ce qui peut avoir un impact très important dans certains domaines.

Outre les services écosystémiques qu'ils fournissent, les forêts et les arbres fournissent de nombreux services de régulation et de soutien qui sont essentiels à l'agriculture (Richardson, 2010; Foli et al., 2014), ainsi qu'aux communautés et à l'humanité.

Les forêts et les arbres jouent un rôle considérable dans le cycle hydrologique, en régulant les flux des eaux de surface et des eaux souterraines tout en contribuant à la qualité de l'eau (Miura et al., 2015; Ellison et al., 2017) et en contribuant aux précipitations, localement et à distance sous le vent (Ellison et al., 2017). En outre, ils facilitent l'infiltration et peuvent améliorer la recharge des eaux souterraines tout en offrant une protection importante contre les inondations, y compris loin en aval. Dans les zones côtières, les forêts de mangroves protègent 18 millions de personnes et constituent des écosystèmes essentiels qui protègent les terres et les communautés côtières contre l'élévation du niveau de la mer, les tempêtes et les inondations, tout en offrant toute une série d'avantages liés à la pêche (Commission mondiale de l'adaptation, 2019). Les forêts et les arbres contribuent à l'accumulation des sols (Kimble et al., 2007) et à leur protection contre l'érosion hydrique et éolienne ainsi qu'à la circulation des nutriments (Bradshaw et al., 2007), notamment par le biais de leur système racinaire.

Les forêts abritent plus de la moitié des espèces végétales et animales terrestres connues dans le monde (Shvidenko et al., 2005; Aerts et Honnay, 2011). Elles comprennent un certain nombre d'espèces qui fournissent des services bénéfiques à différentes échelles spatiales, comme les pollinisateurs et les ennemis naturels des parasites (Foli et al., 2014). La pollinisation est l'un des services écosystémiques les plus fréquemment étudiés. En fait, de nombreuses études ont montré un lien entre la proximité des forêts et des parcelles forestières et la densité des pollinisateurs sauvages qui profitent aux rendements et à la qualité des cultures pollinisées (Garibaldi et al., 2016).

Outre ces avantages économiques et environnementaux, les forêts sont également porteuses de valeurs sociales, culturelles et religieuses qui sont d'une importance capitale pour de nombreuses communautés et groupes, notamment dans le cas des populations autochtones où les forêts sont non seulement essentielles à leurs moyens d'existence, mais aussi à leur culture, leurs traditions, leur religion, leurs croyances et leurs pratiques spirituelles (par exemple Widmark, 2009).

L'approvisionnement, la régulation, le soutien (et même) les services écosystémiques culturels fournis par les forêts et les arbres peuvent constituer des atouts essentiels pour les paysages, les communautés et les ménages dans le contexte du changement climatique. Le changement climatique ne manquera pas d'amplifier un certain nombre de risques affectant les systèmes agricoles, les paysages, les communautés et les ménages (par exemple, FAO 2016a) pour lesquels les forêts et les arbres peuvent servir de tampon, réduisant ainsi les effets des chocs. Cela justifie que, dans le contexte du changement climatique et lors de l'élaboration de plans d'adaptation, les forêts et les arbres et le rôle qu'ils jouent dans la résilience des paysages, des communautés et des 
ménages face à divers chocs et risques méritent une attention particulière. À bien des égards, elles offrent des options sans regret et toute une série de filets de sécurité.

Par exemple, les services écosystémiques fournis par les forêts et les arbres sont particulièrement importants pour réduire les impacts de la variabilité climatique et des chocs liés aux conditions météorologiques, tels que les inondations, les sécheresses, les vents et les vagues de chaleur, sur les activités économiques, les infrastructures et les populations (voir les exemples au point 6.3). Dans les systèmes agroforestiers, les arbres peuvent contribuer à la régulation du microclimat, en augmentant la productivité et la résilience du système de production alimentaire (Pramova et al., 2012).

Les arbres contribuent à une diversification des sources de nourriture et de revenus qui peut aider à amortir les chocs économiques induits par le changement climatique. En outre, des systèmes diversifiés sont susceptibles de fournir une plus grande résilience à une volatilité accrue de l'approvisionnement et des prix des denrées alimentaires (Vira et al., 2015), qu'elle soit liée au climat ou non. Dans de nombreuses communautés, les aliments forestiers (y compris le gibier sauvage) sont utilisés comme filet de sécurité pendant la période de soudure ou de famine ou en cas de mauvaises récoltes (Blackie et al., 2014; Keller et al., 2006; Shackleton et Shackleton, 2004; Sunderland et al., 2013; Karjalainen et al., 2010, Koffi et al., 2017). Par exemple, en République-Unie de Tanzanie, une plus grande partie du régime alimentaire a été constituée d'aliments sauvages pendant les périodes d'insécurité alimentaire (Powell et al., 2013b). Dans une revue de 9 études comprenant une évaluation sur plusieurs saisons, six cas ont montré une plus grande dépendance aux aliments sauvages pendant la période de soudure ou d'insécurité alimentaire, tandis que les trois autres cas ont montré une plus grande utilisation d'aliments sauvages lorsque ceux-ci étaient les plus disponibles (Powell et al., 2015). Dans les régions semi-arides, comme au Sahel, pendant la saison sèche, les arbres et les arbustes fournissent du fourrage pour le bétail (Franzel et al., 2014). En outre, les forêts et les arbres sont utilisés comme filet de sécurité pour répondre à des besoins occasionnels de revenus, notamment en période de conflit, de catastrophe naturelle ou de crise économique. Les hommes et les femmes les plus vulnérables peuvent trouver une source de revenus et d'emploi grâce à la collecte et à la vente de bois de chauffage et de produits forestiers non ligneux (NWFP) (Angelsen et Wunder, 2003; Shackleton et Shackleton, 2004; Mulenga et al., 2012).

La reconnaissance de cette fonction tampon des forêts et des arbres dans les paysages (van Noordwijk et al., 2011), qui protège les populations des dangers et des chocs liés au climat, a conduit à proposer le concept de «services d'adaptation au climat»(Lavorel et al., 2015), défini comme «les avantages pour les personnes d'une capacité sociale accrue à répondre au changement, fournie par la capacité des écosystèmes à modérer et à s'adapter au changement climatique et à la vulnérabilité».

Ces fonctions tampon des forêts et des arbres vont bien au-delà de l'agriculture. Elles sont essentielles à l'adaptation des terres et des systèmes d'eau grâce à la fourniture et à la régulation des flux d'eau, en quantité et en qualité, localement et à distance, assurant ainsi une protection contre les inondations, l'érosion, les glissements de terrain et l'élévation du niveau de la mer. En outre, elles représentent un grand potentiel pour l'adaptation des villes car elles fournissent un effet de refroidissement qui deviendra encore plus précieux avec les vagues de chaleur de plus en plus fréquentes dans les zones urbaines.

\subsection{Impacts du changement climatique sur les forêts et les arbres}

Les risques que le changement et la variabilité climatiques font peser sur les forêts et les arbres sont bien connus. Le changement climatique peut avoir des effets directs et indirects sur les forêts et les arbres. Nous qualifions ici d'impacts directs ceux qui sont directement causés par une modification des caractéristiques physiques telles que la température ou les précipitations. Les effets indirects sont ceux qui affectent la production et/ou l'état par des changements dans d'autres espèces telles que les pollinisateurs, les parasites indigènes, les vecteurs de maladies, les espèces envahissantes (insectes nuisibles, agents 
pathogènes, plantes et vertébrés).

Les effets directs sont plus faciles à prévoir car ils peuvent être plus facilement simulés et/ou modélisés. Ils sont maintenant assez bien projetés pour certaines espèces d'arbres et certains types de forêts simples. Les effets indirects du changement climatique sur les forêts et les arbres sont beaucoup plus difficiles à modéliser étant donné le grand nombre de facteurs en interaction qui déterminent la santé et l'état des forêts. Dans certains cas, soit une référence à un système comparable dans le cadre du climat prévu, soit l'observation des impacts d'un changement climatique comparable sur un autre système peut être utile pour la prévision des impacts (FAO, 2016a).

Des effets négatifs sont déjà visibles en de nombreux endroits, souvent combinés à d'autres stress (Braatz, 2012; Keenan, 2015). Des preuves récentes suggèrent que dans un large éventail de systèmes forestiers, le réchauffement et les changements dans les précipitations augmentent la mortalité des arbres en raison du stress thermique, du stress de la sécheresse et des invasions de ravageurs (Allen et al., 2010). De nombreuses zones de forêts boréales ont connu des baisses de productivité qui ont été attribuées à la sécheresse induite par le réchauffement (Williams et al., 2013). Le réchauffement et l'assèchement, associés au déclin de la productivité, aux perturbations causées par les insectes et à la mortalité des arbres qui y est associée, favorisent également une plus grande perturbation par le feu (Settele et al., 2014). La tendance générale pour les forêts tempérées a été jusqu'à récemment une augmentation des taux de croissance, en raison d'une combinaison de l'augmentation de la durée de la saison de croissance, de l'augmentation des dépôts atmosphériques de $\mathrm{CO} 2$ et d'azote et de la gestion des forêts (Ciais et al., 2008). Cependant, des indications récentes montrent des signes de stress climatique avec une augmentation de la mortalité des arbres, des changements dans le régime des incendies, des invasions d'insectes et des attaques de pathogènes (Settele et al., 2014). On a également noté une diminution significative de la régénération des arbres après un incendie dans les montagnes Rocheuses américaines (StevensRuman et al., 2018). Les tempêtes et les vents violents, les inondations et les crues ainsi que les glissements de terrain et les avalanches affectent également les forêts. Dans les zones côtières, les forêts, en particulier les mangroves, sont touchées par l'intrusion d'eau salée et l'élévation du niveau de la mer, ainsi que par les dommages causés par les tempêtes côtières.

La plupart des espèces d'arbres ont une aire de répartition climatique dans laquelle elles poussent le mieux, sont en concurrence avec d'autres espèces de plantes, peuvent s'adapter aux changements environnementaux et réagir à l'augmentation des attaques d'insectes, des maladies, des conditions environnementales défavorables et des influences anthropiques. Certaines espèces s'adapteront mieux que d'autres à l'évolution des conditions, ce qui entraînera des changements dans la composition des forêts plutôt que des changements géographiques dans les types de forêts (Breshears et al., 2008). En général, les espèces d'arbres sont susceptibles de se déplacer vers des latitudes ou des altitudes plus élevées en raison du réchauffement climatique (Rosenzweig et al., 2007; Breshears et al., 2008). De tels déplacements peuvent se produire sur des distances assez courtes le long de gradients altitudinaux (par exemple, en montagne), tout en nécessitant des déplacements de centaines de kilomètres dans des zones plates. Les modèles prédisent que l'espace climatique potentiel pour la plupart des espèces d'arbres se déplacera vers les pôles et à des altitudes plus élevées plus rapidement que la migration naturelle. Les forêts boréales devraient se déplacer vers le nord en raison du changement climatique, tandis que les forêts tempérées s'étendront vers le nord et dans une plus grande mesure que les forêts boréales; par conséquent, la superficie totale des forêts boréales sera réduite (Burton et al., 2010). En retour, les changements dans la composition des espèces d'arbres sont un des principaux moteurs des changements de la productivité des forêts dans le cadre du changement climatique (Morin et al., 2018).

On s'attend à ce que les effets du changement climatique, du changement d'affectation des terres et des pratiques non durables d'utilisation des terres interagissent les uns avec les autres (FAO, 2017c). Les changements dans la disponibilité de l'eau seront un facteur clé pour la survie et la croissance de nombreuses espèces forestières, bien que la réponse aux sécheresses prolongées varie selon les espèces et aussi selon les variétés d'une même espèce (Lucier et al., 2009). Le changement climatique augmentera le risque d'incendies 
fréquents et plus intenses, en particulier dans les zones où il entraîne une diminution des précipitations ou un allongement des périodes sèches, comme dans le cas des forêts boréales (Burton et al., 2010), des forêts des régions méditerranéennes et subtropicales (Fischlin et al., 2009), en plus des zones où les pratiques traditionnelles de défrichement par le feu sont utilisées, comme en Amazonie (Aragão et al., 2008; Nepstad et al., 2008). Les forêts tropicales humides abritent de nombreuses espèces qui sont vulnérables à la sécheresse et à la mortalité due aux incendies pendant les périodes de sécheresse extrême. La fréquence et la gravité des incendies de forêt augmentent en raison de la combinaison du changement d'affectation des terres et de la sécheresse, notamment en Amazonie. Le changement climatique, la déforestation, la fragmentation, les incendies et la pression humaine font que pratiquement toutes les forêts tropicales sèches risquent d'être remplacées ou dégradées (Miles et al., 2006).

Le changement climatique peut accroître l'impact des insectes ravageurs en leur permettant de s'établir dans des zones où ils ne pouvaient pas le faire auparavant; en fait, les effets du changement climatique sur les insectes forestiers ont été démontrés pour un certain nombre d'espèces et de guildes (Pureswaran et al., 2018). Les changements de température peuvent entraîner des modifications de l'aire de répartition géographique et faciliter l'hivernage, permettant à certaines espèces d'étendre leur aire de répartition géographique vers le pôle et vers des altitudes plus élevées (Porter et al., 2014; Svobodová et al., 2014). Par exemple, la propagation du dendroctone du pin ponderosa (Dendroctonus ponderosae) dans les forêts boréales a été largement attribuée à l'absence de températures constamment basses sur de longues périodes, ce qui a permis à des foyers déjà existants de se propager dans les zones montagneuses et dans les forêts boréales plus froides (Burton et al., 2010). La noctuelle processionnaire du pin, Thaumetopea pityocampa, est un insecte modèle indicateur du réchauffement climatique, par son expansion vers le nord et vers le haut de son aire de répartition (Roques et al., 2015). On s'attend à une augmentation des infestations de pourriture des racines et des bourgeons dans les forêts de conifères finlandaises en raison d'un champignon virulent, Heterobasidion parviporum, dont la propagation est favorisée par des périodes de récolte plus longues, des dégâts accrus dus aux tempêtes et une saison de production de spores plus longue (Burton et al., 2010). Sous les tropiques, le réchauffement accru accélère le cycle de vie de nombreux insectes nuisibles et l'augmentation des dommages causés par les incendies rend les arbres plus sensibles aux attaques d'insectes, ce qui accélère à son tour le cycle de vie de ces nuisibles (Lucier et al., 2009). Le changement climatique peut également accroître l'impact des insectes ravageurs en leur permettant d'apparaître plus tôt dans la saison en raison des températures plus élevées. En outre, des études prévoient un nombre accru de générations par an sous l'effet du changement climatique, comme pour le nématode du caféier au Brésil (Ghini et al., 2008) et les parasites des noix en Californie (Luedeling et al., 2011).

En dépit de leur diversité, les forêts et les systèmes arboricoles très divers partagent certains défis communs en matière d'adaptation au changement climatique. Les arbres ont un cycle de vie qui s'étend souvent sur des dizaines ou des centaines d'années, ils doivent donc être adaptés à la fois au climat actuel et au climat futur à long terme. Compte tenu de leur longévité, ils subiront davantage de variabilité et de chocs que les autres plantes, en outre, ils doivent être plus résistants à un ensemble de conditions plus diverses. Par rapport aux cultures annuelles, les arbres sont plus difficiles et plus longs à domestiquer et à multiplier, ce qui doit être pris en compte dans l'évolution des forêts gérées et plantées. Les forêts naturelles ont également tendance à être des systèmes complexes avec de multiples interactions entre les espèces qui peuvent être modifiées par le changement climatique, souvent de manière inattendue, ce qui rend l'adaptation au changement climatique plus difficile. Enfin, et c'est peut-être le plus important, l'adaptation des forêts et des arbres prend du temps, nécessite des efforts à long terme, des politiques appropriées et une sécurité d'occupation. 


\subsection{Relations entre les forêts et les systèmes vulnérables}

L'objectif de cette section est d'examiner les secteurs identifiés par les pays comme étant les plus vulnérables au changement climatique et d'étudier comment les forêts et les arbres peuvent contribuer à leur résilience et à leur adaptation.

Les secteurs les plus fréquemment identifiés comme vulnérables dans les PANA préparés par les pays les moins avancés (CCNUCC, 2018) étaient:

- l'agriculture

- l'eau

- la sécurité alimentaire

- les infrastructures (y compris les infrastructures côtières, les bâtiments, les transports et les communications)

- les écosystèmes et la biodiversité

- la santé

Ont également été identifiés:

- les zones vulnérables: zones côtières, zones rurales, villes, vallées fluviales et oasis ;

- les populations vulnérables: les populations rurales, les communautés côtières, les populations pauvres et les femmes.

La plupart des pays qui ont inclus une composante d'adaptation dans leur CDN ont identifié des secteurs et des zones particulièrement vulnérables (CCNUCC, 2015). Ces secteurs et zones ont été résumés comme suit:

- l'eau

- l'agriculture et la foresterie

- les écosystèmes et la biodiversité, y compris la faune et la flore sauvages

- la santé

- l'énergie, le tourisme, les infrastructures et les établissements humains

- les zones sujettes à la sécheresse et à la désertification, les zones côtières de faible altitude et les petites îles

- les pays enclavés et montagnes

- les populations vulnérables

- les populations rurales
- les segments les plus pauvres de la société

- les femmes, les jeunes, les personnes âgées et les handicapés

Ces secteurs informent également la structure de la plupart des PNA, avec quelques modifications de portée qui sont généralement dues à des spécificités nationales et liées aux responsabilités des ministères concernés. Si l'on considère ces secteurs comme des systèmes, il apparaît que, dans la plupart des cas, les forêts contribuent déjà de manière significative à leur résilience et que leur rôle deviendra de plus en plus important avec le changement climatique.

Le potentiel des forêts et des arbres à contribuer à l'adaptation grâce aux services écosystémiques qu'ils fournissent a été amplement reconnu par les PANA. En fait, un examen des projets prioritaires proposés dans les PANA a révélé l'importance de la foresterie, non seulement en soi, mais aussi pour le soutien à l'adaptation d'autres secteurs (Meybeck et al., 2012). Les PANA comprennent des projets traitant directement des forêts, par exemple, l'aide à la mise en œuvre de plans de gestion forestière communautaire ou la gestion et la prévention des incendies de forêt au niveau communautaire. En outre, la foresterie fait très souvent partie de projets intégrés pour l'amélioration de la gestion des bassins versants, la prévention de l'érosion des sols, des glissements de terrain et des inondations. L'importance des services écosystémiques a été reconnue dans plus de 50 pour cent des PANA, les forêts et les arbres étant les plus souvent cités (Pramova et al., 2012). Environ 22 pour cent des projets proposés dans les PANA comprennent des activités écosystémiques pour le bien-être social ou l'adaptation, la plupart d'entre elles étant destinées à soutenir d'autres mesures d'adaptation (par exemple, les infrastructures) (Pramova et al., 2012). Les projets forestiers sont souvent décrits comme bénéficiant à plusieurs secteurs, par exemple, la restauration des mangroves à Djibouti devrait contribuer à la reconstitution des réserves de poissons, à la lutte contre l'érosion en vue d'une amélioration de l'agriculture, ainsi qu'à l'adaptation des zones côtières et du secteur de l'eau grâce à une protection des établissements humains contre les tempêtes, un contrôle de l'intrusion d'eau salée et une contribution à la recharge des nappes phréatiques. Un projet au Burkina Faso pour l'amélioration de la gestion de la végétation naturelle et la promotion des produits forestiers 
non ligneux devrait apporter des avantages supplémentaires en matière d'alimentation, de revenus et de santé. L'étude conclut en invitant à adopter une perspective plus large en matière d'adaptation basée sur les écosystèmes (AbE).

Les forêts, les arbres et l'agroforesterie peuvent jouer un rôle important dans l'adaptation de la plupart des secteurs identifiés par les pays comme les plus vulnérables. Les contributions potentielles des forêts, des arbres et de l'agroforesterie sont symbolisées par les flèches de la figure 3. Une grande partie de ce potentiel et des actions connexes devra être mise en œuvre dans la pratique au niveau local, dans le paysage, par le biais de l'AbE; toutefois, il faudra que des conditions favorables soient mises en place au niveau national.

\section{FIGURE 3.}

\section{Contributions potentielles des forêts, des arbres et de l'agroforesterie à} l'adaptation d'autres secteurs/systèmes

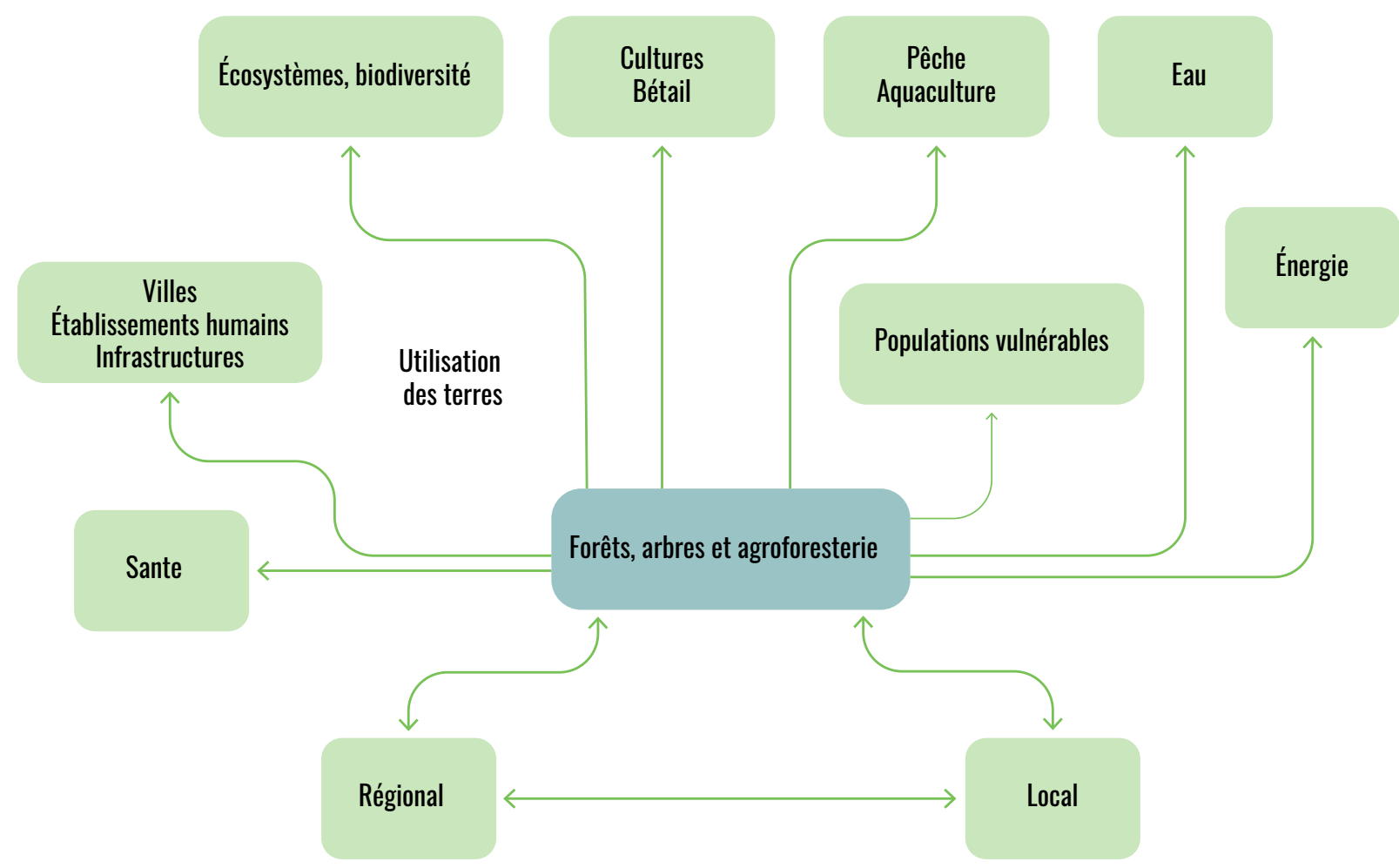

Ces flèches représentent des contributions physiques, biologiques et/ou économiques. Chaque secteur peut également être considéré comme un système, avec ses propres acteurs et institutions. Comme le résume la figure, ces secteurs correspondent généralement aux périmètres des ministères de tutelle, auxquels il convient d'ajouter les institutions régionales et locales, qui peuvent être soit des émanations des administrations nationales, soit des administrations locales, selon les pays. Dans la lecture de la figure, les flèches représentent les liens à bâtir entre les systèmes, les acteurs et les institutions dans le cadre du PNA afin de renforcer les contributions potentielles des forêts, des arbres et de l'agroforesterie à l'adaptation d'autres secteurs et, en définitive, de l'ensemble du pays. 


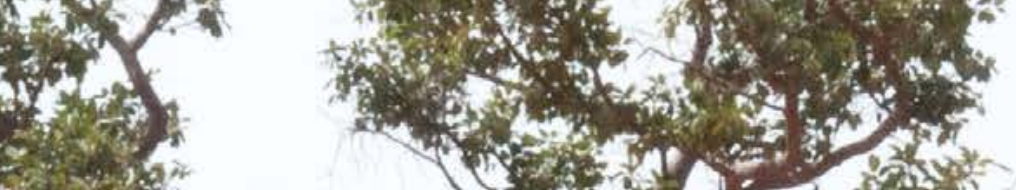
(1)

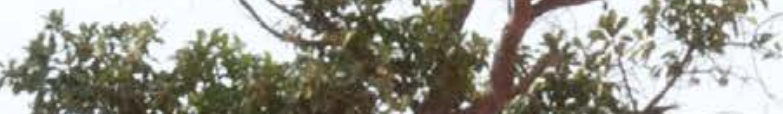

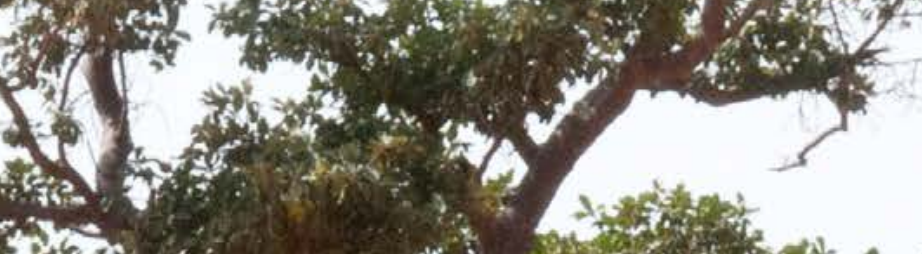

thi.

$x^{2}$

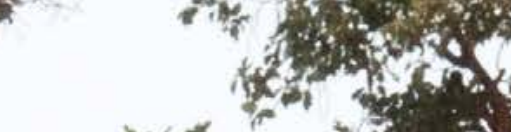

(3)

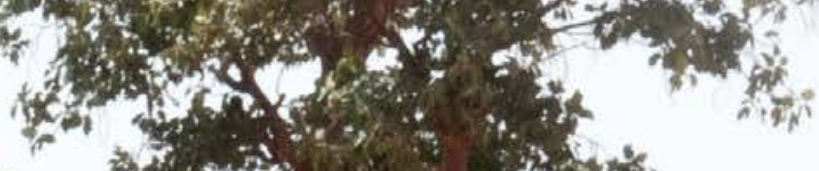

$6 k x=2 w^{2}$

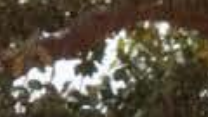

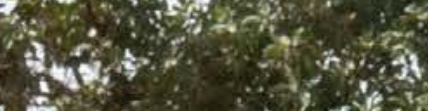
Mintexts
(t)
(s)
(1)

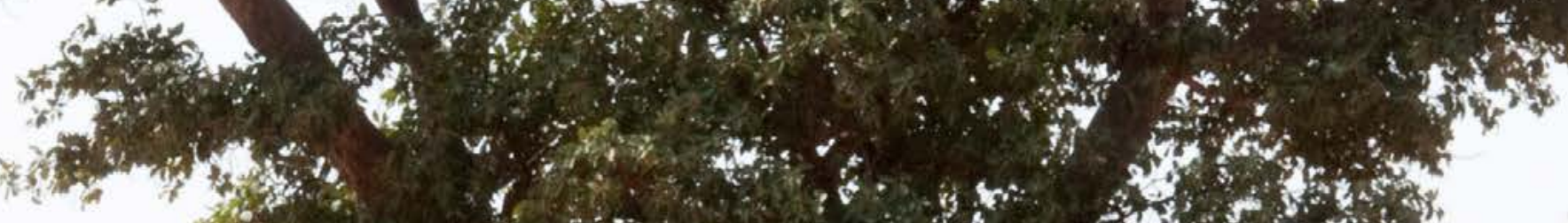

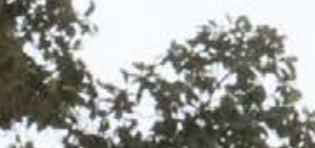 3.
$+\lim _{2}$ is
$x_{i}$

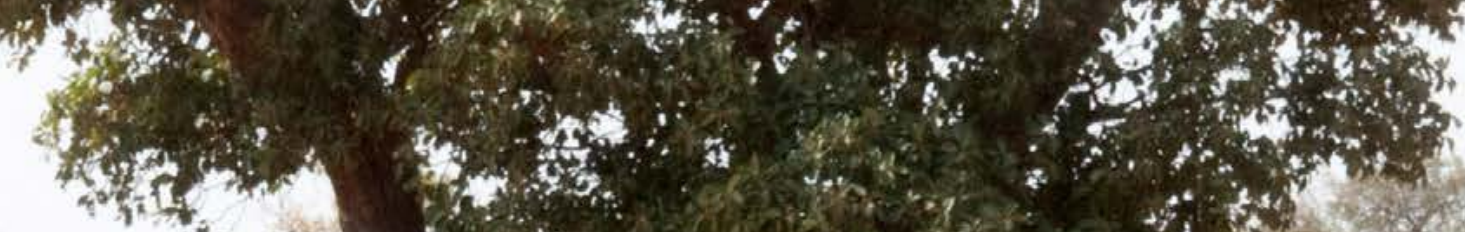

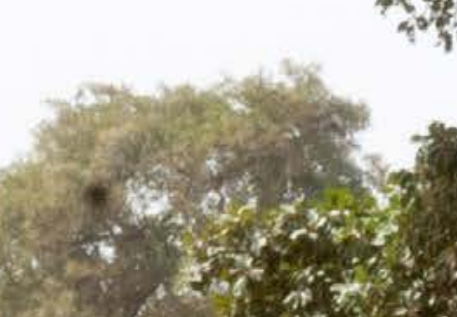

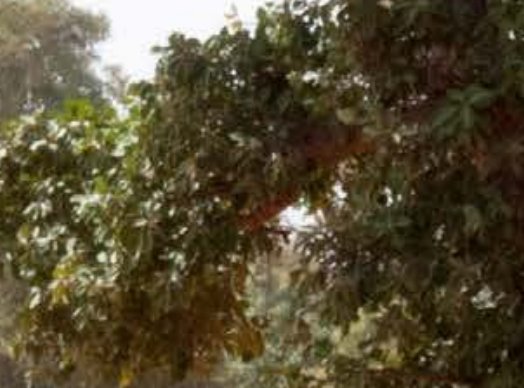
sinis:
+7 is
Whothents
sing whith
1.

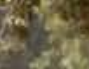

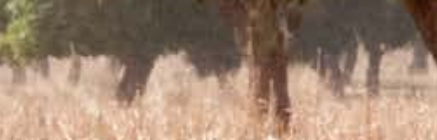
(a) inting

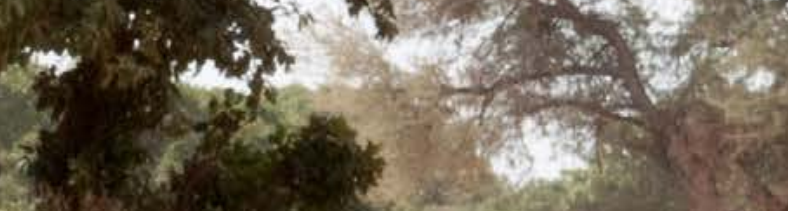
tont?
$-\frac{1}{3}+\frac{1}{3}$
$6 x^{3}, 2$
ovis

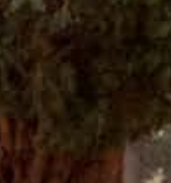

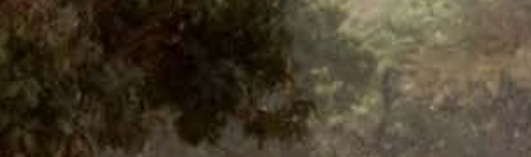

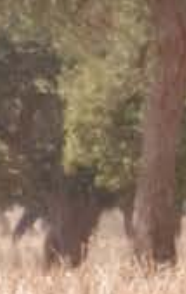
ace nin

4. 790 ing

$\operatorname{los}^{2+\infty}$

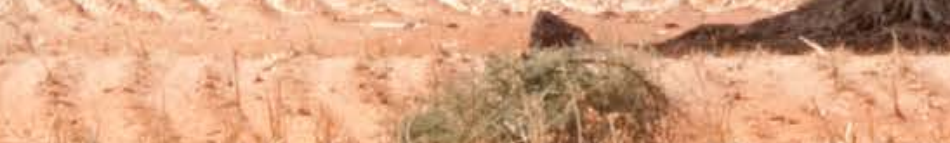

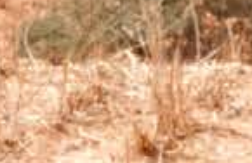

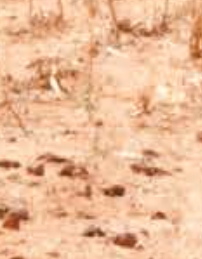

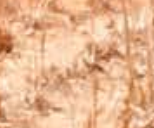
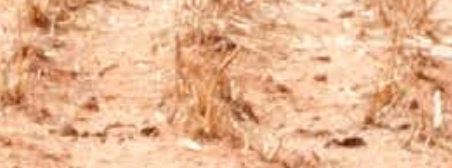

Mc

2

$$
4-a x-2 x
$$$$
392
$$$$
y^{4}
$$$$
\text { 19. } 48
$$$$
\text { - in }
$$

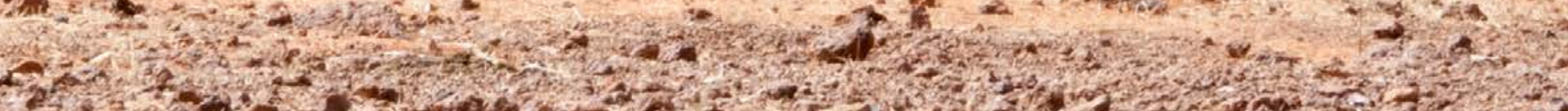

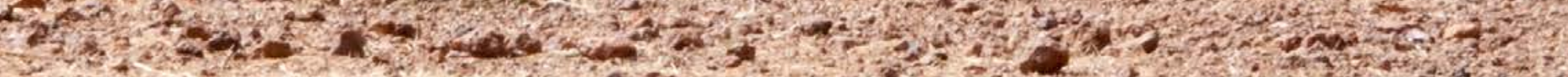

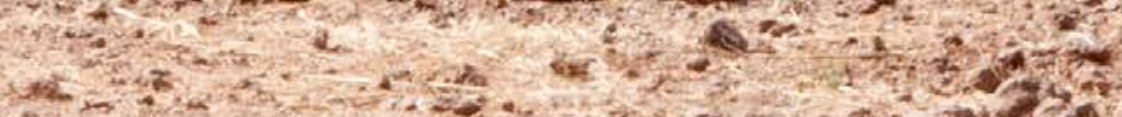




\section{- Dispositions institutionnelles pour prendre en compte les forêts et les arbres dans les PNA}

\author{
L'objectif de \\ cette section est \\ de fournir des \\ recommandations \\ sur les moyens \\ possibles pour le \\ secteur forestier de \\ s'engager au mieux \\ dans le processus \\ en fonction \\ des spécificités \\ institutionnelles \\ nationales (en \\ s'appuyant sur la \\ section 2.4).
}

\begin{abstract}
Les deux grands axes identifiés ci-dessus, à savoir le potentiel d'adaptation des forêts et des systèmes arboricoles aux effets du changement climatique et la contribution de ces systèmes à une résilience accrue dans un certain nombre de secteurs clés particulièrement vulnérables aux effets du changement climatique, nécessitent deux dispositifs institutionnels différents et complémentaires: i) au sein du secteur forestier; et ii) en relation avec les autres sous-secteurs agricoles ainsi qu'avec d'autres secteurs pertinents, notamment la gestion de l'eau, l'énergie, l'aménagement du territoire, etc.
\end{abstract}

Ce double processus devrait être intégré dans le processus global du PNA lui-même et s'appuyer autant que possible sur les organisations et les mécanismes existants. Il devrait être dirigé par le point focal foresterie du PNA. En outre, il doit être soutenu par des mécanismes de communication appropriés afin de faciliter l'engagement des acteurs des forêts et des systèmes arboricoles et d'assurer la circulation des informations en provenance et à destination des différentes institutions en charge du PNA.

\subsection{Mise en place d'un processus d'adaptation pour les forêts et les arbres}

Étant donné l'ampleur des effets du changement climatique sur les forêts et sur les populations qui en dépendent (voir encadré 6), il est nécessaire de mettre en place un processus spécifique au secteur forestier afin de comprendre les vulnérabilités et les moyens potentiels d'adaptation. Ce processus devrait être aligné et intégré dans le programme forestier national (pfn) du pays, ou dans le processus qui fournit un cadre pour la mise en œuvre de la gestion durable des forêts et de leurs contributions au développement durable (FAO, 2018a).

Un processus d'analyse des vulnérabilités au changement climatique et des mesures d'adaptation dans le secteur forestier doit tenir compte de la diversité des situations, même au niveau national, de la diversité des forêts et des systèmes arborés, des personnes qui en dépendent, des relations entre les populations et les forêts, et de la diversité des priorités. Il devrait associer tous les acteurs concernés et s'appuyer 
sur une combinaison d'approches descendantes et ascendantes pour faciliter les échanges entre la communauté scientifique et les connaissances, les préoccupations et les capacités des acteurs locaux.

Ce processus sectoriel devrait avoir trois objectifs principaux: (i) sensibiliser les populations à la vulnérabilité des forêts et des arbres au changement climatique, aux risques liés à la perte ou à la dégradation de ces ressources, ainsi qu'aux stratégies d'adaptation qui peuvent être mises en place pour les forêts et les arbres afin de maximiser leur contribution à la bonne mise en œuvre du PNA; (ii) soutenir les étapes du processus du PNA, en fournissant des données, des informations et des connaissances sur le secteur, sur ses vulnérabilités, sur ce qui est nécessaire pour permettre son adaptation et sa contribution à l'adaptation d'autres secteurs; et (iii) suivre la préparation et la mise en œuvre du plan, évaluer les impacts potentiels de ses mesures sur la foresterie et sur d'autres secteurs et proposer des améliorations le cas échéant. Le processus sectoriel devrait être bien lié au processus national global et utiliser autant que possible les structures et institutions existantes pour contribuer à l'intégration de l'adaptation.

Pour atteindre les objectifs mentionnés cidessus, le processus devra associer tous les acteurs nationaux concernés ainsi que diverses compétences scientifiques et techniques. Il devra également associer les acteurs locaux, à la fois pour recueillir des informations et pour faciliter leur engagement dans les processus locaux. Il pourrait par exemple s'appuyer sur un groupe national, auquel pourraient être associés des points focaux provinciaux ou régionaux, par exemple des agents forestiers provinciaux ou régionaux, qui pourraient eux-mêmes, au besoin, organiser une concertation au niveau provinciale ou régionale. En fonction de la manière dont le processus du PNA est organisé, le processus sectoriel au niveau local/régional sera plus ou moins développé. Il est cependant toujours nécessaire, pour la Foresterie, d'avoir un bon lien avec les niveaux locaux étant donné les spécificités locales du secteur et étant donné également que c'est au niveau du paysage que ses interactions avec d'autres secteurs peuvent être le mieux comprises et les mesures mises en œuvre.

Plusieurs points sont particulièrement importants pour le secteur afin d'orienter la composition de ces groupes. L'un des principes fondamentaux du processus du PNA est l'inclusion et la transparence.
Cela nécessite une large participation qui inclurait les femmes, les populations autochtones et tous les groupes vulnérables et marginalisés, d'autant plus qu'ils sont particulièrement vulnérables et souvent partiellement ou totalement dépendants de la forêt (voir encadré 7). Une participation aussi large peut également contribuer à rendre plus visibles les contributions des forêts aux moyens d'existence et au bien-être humain, contributions qui ne sont pas toujours prises en compte dans les statistiques économiques. À cet égard, il est également utile d'associer tous les groupes ayant un intérêt dans les forêts et les arbres même lorsqu'ils n'en dépendent pas économiquement. Outre les acteurs forestiers «traditionnels», les propriétaires, gestionnaires et utilisateurs de forêts doivent également être associés aux acteurs impliqués dans les forêts urbaines, l'agroforesterie, l'horticulture, ainsi que dans les chaînes de valeur pertinentes pour le cacao, le café, le caoutchouc, le beurre de karité et d'autres produits arboricoles, car ils partagent des intérêts et des préoccupations communs. Étant donné que les décisions en matière d'adaptation doivent autant que possible être fondées sur une analyse économique solide, il est nécessaire de veiller à ce que des compétences en économie soient présentes ou puissent être mises à contribution.

Compte tenu de ce qui précède, un groupe national pourrait inclure des représentants:

- des gestionnaires de forêts, privés et publics, y compris des forêts communautaires;

- des services de vulgarisation forestière (publics et privés);

- des communautés dépendantes de la forêt;

- des propriétaires et «détenteurs» de forêts (avec des droits de gestion à long terme), différentes catégories et échelles;

- des utilisateurs de la forêt (y compris avec des droits d'utilisation informels);

- des producteurs de produits forestiers, bois et non bois;

- des transformateurs et les distributeurs de produits forestiers;

- des producteurs, transformateurs et distributeurs de produits arboricoles et associés (café, cacao, caoutchouc, horticulture, huile de palme);

- des organisations impliquées dans la conservation et la multiplication des ressources génétiques;

- des organisations agricoles forestières;

- des agriculteurs pratiquant l'agroforesterie; 
- de la société civile et des ONG;

- des autorités de planification de l'État, des districts ou des collectivités locales (y compris les villes);

- des universités et de la recherche.

En fonction des spécificités de la Foresterie, de l'agroforesterie et des cultures arboricoles dans le pays, il peut être utile d'organiser des groupes spécifiques axés sur des aspects particuliers, par exemple une chaîne de valeur associant tous les acteurs depuis la production jusqu'à la transformation et la distribution, ou sur des questions spécifiques comme les ressources génétiques, depuis l'identification et la conservation jusqu'à la distribution du matériel génétique approprié. D'autres groupes spécialisés, tels que l'énergie, la biodiversité ou l'eau pourraient suivre de plus près et contribuer aux travaux de sections spécifiques du PNA. Pour garantir la crédibilité, la transparence et l'ouverture du processus, il est nécessaire de le formaliser dans une certaine mesure, en publiant une liste des personnes impliquées et un compte rendu écrit ou des listes de points de décision après chaque réunion.

Les acteurs impliqués dans le processus peuvent également avoir besoin d'une certaine formation, au moins sur le processus du PNA lui-même. En outre, de nouvelles connaissances, compétences et expertises peuvent être nécessaires pour participer efficacement au processus et à sa mise en œuvre. Les gestionnaires de forêts et les autres parties prenantes devraient disposer de connaissances et d'une expertise suffisante pour leur permettre d'entreprendre des évaluations de la vulnérabilité et des risques, de concevoir et de réviser les plans de gestion, de mettre en œuvre des actions d'adaptation au changement climatique et de surveiller les impacts du changement climatique et les résultats des actions menées dans ce domaine. En fait, ces activités de renforcement des capacités font également partie du PNA et sont essentielles à sa mise en œuvre.

\subsection{Assurer la liaison avec les autres sous-secteurs de l'agriculture}

La Foresterie, les cultures, l'élevage, la pêche et l'aquaculture ont beaucoup en commun. Elles sont particulièrement dépendantes des conditions climatiques. Elles dépendent toutes de l'utilisation des ressources naturelles, en particulier de la terre et de l'eau, pour lesquelles elles sont parfois en compétition. Elles partagent également de nombreuses caractéristiques économiques et sociales, notamment le nombre de personnes qui en dépendent et leur importance particulière pour les LCD. Les populations rurales dépendent souvent, pour leur subsistance, d'une combinaison d'activités dans les différents sous-secteurs, les forêts étant souvent la dernière option pour ceux qui ont tout perdu. Par conséquent, les risques liés au changement climatique auxquels ces secteurs sont confrontés représentent des défis communs. Des solutions communes sont disponibles, telles que les systèmes de surveillance du temps et des écosystèmes (y compris les parasites et les maladies) ou la conservation et l'utilisation durable des ressources génétiques par exemple. Il existe également des problèmes communs, comme le besoin de gérer les synergies et les compromis en matière de gestion de l'eau et d'aménagement du territoire. En particulier, le développement de l'agriculture a un impact direct sur les forêts car il est le principal moteur de la déforestation, tandis que les forêts et les arbres contribuent de manière importante à la résilience des autres activités agricoles.

Par conséquent, l'établissement d'échanges réguliers entre les sous-secteurs agricoles (cultures, élevage, forêts, pêche et aquaculture) pour favoriser la collaboration et les progrès vers des approches communes en matière d'adaptation est fondamental. En outre, ces échanges pourraient contribuer à la mise en place d'approches intégrées au niveau du paysage. Au niveau national, des réunions régulières pourraient être organisées entre les participants des sous-secteurs agricoles et les différents axes de travail pour échanger des informations, élaborer des stratégies communes sur des questions transversales et élaborer des propositions conjointes sur des questions intersectorielles d'intérêt commun. Des réunions plus larges pourraient rassembler tous les membres des groupes travaillant respectivement sur la Foresterie, l'agriculture, la pêche et l'aquaculture. Ces réunions permettraient de faire le point sur l'état d'avancement du PNA et de soutenir les étapes cruciales de sa préparation, de sa mise en œuvre et de sa révision. Ces rassemblements pourraient également comprendre des sessions consacrées à l'apprentissage, au renforcement des capacités et à l'échange des enseignements tirés. Au niveau infranational, ces réunions pourraient ouvrir la voie à la mise en œuvre du PNA au niveau du paysage, à différentes échelles, y compris par le biais de l'AbE. 
4.3 Organiser la participation du secteur forestier à la planification de l'adaptation nationale, notamment en clarifiant les mandats et en faisant participer les parties prenantes

Cette étape consiste à comprendre le processus du PNA établi au niveau national, afin d'identifier comment le secteur des forêts et des arbres peut apporter une contribution appropriée et interagir avec d'autres secteurs. Le mandat et la composition de certains groupes sectoriels spécifiques peuvent nécessiter des éclaircissements. Il s'agit également d'identifier les acteurs à engager dans la réflexion et la planification du secteur (voir également le tableau 4). Une attention particulière doit être accordée à l'inclusion des femmes, des populations autochtones et des groupes marginalisés, souvent les plus dépendants des forêts, surtout en temps de crise. Il est également important de ne pas oublier les groupes qui s'intéressent aux forêts, même de manière indirecte, comme les pêcheurs de mangroves par exemple. Il est nécessaire d'organiser une représentation appropriée du secteur forestier dans le processus global du PNA ainsi que dans les processus sectoriels qui concernent les forêts et les arbres ou pour lesquels les forêts et les arbres peuvent être pertinents, comme la gestion de l'eau, l'aménagement du territoire ou l'énergie.

La participation du secteur forestier au processus a les principaux objectifs suivants:

- accroître le niveau de compréhension de tous les acteurs investis dans l'adaptation des spécificités des forêts et des arbres, ainsi que des défis et des opportunités qui y sont liés (comme décrit au point 4.2), par exemple en attirant l'attention de la communauté de la conservation sur la nécessité de prendre en compte le rôle des forêts pour les moyens d'existence indigènes et locaux;

- veiller à ce que les besoins du secteur soient dûment pris en considération au niveau national et dans les stratégies et politiques des autres secteurs;

- promouvoir et renforcer le rôle des forêts et des arbres en tant que moyen d'adaptation.

La première étape consiste à avoir une idée précise des différents volets du processus global du PNA et de leurs mandats. Comme il n'y a pas souvent de volet de travail consacré uniquement aux forêts et aux arbres, il est important d'identifier celui qui a le plus de potentiel pour un accent plus fort sur les forêts ainsi que ceux qui sont pertinents, soit parce qu'ils traitent de certaines souscatégories (plantations, horticulture, foresterie urbaine...), soit parce qu'ils ont un impact sur les forêts, soit encore en raison de l'importance de la foresterie pour leur propre adaptation (eau, énergie, établissements humains). Par exemple, les plantations peuvent être traitées dans le cadre d'un axe de travail sur l'agriculture, l'élevage et les plantations comme au Chili, tandis que les forêts naturelles peuvent faire partie d'un axe de travail sur la biodiversité. Dans d'autres cas, les plantations peuvent faire partie des cultures de rente (Sri Lanka). L'agroforesterie est généralement traitée dans le cadre de l'agenda de l'agriculture. La foresterie urbaine serait mieux traitée dans un groupe sur les établissements humains et l'urbanisme. La lutte contre l'envasement des barrages pourrait être traitée soit dans le groupe sur l'eau et/ou dans le groupe sur l'énergie. Il est essentiel que le secteur forestier soit au courant de toutes ces discussions afin de pouvoir profiter des possibilités de sensibilisation au potentiel des forêts et des arbres pour contribuer à l'adaptation.

Pour chacun de ces axes de travail, il convient d'identifier la composition (formalisée ou non) du groupe qui dirige les activités, ainsi que ses modalités de travail (périodicité des réunions, moyens habituels de collecte et de partage des informations), et de savoir s'il s'appuie principalement sur des rapports antérieurs, s'il a lancé des études spécifiques et s'il accueille des contributions extérieures. L'accent sera généralement mis sur le domaine de travail le plus actif en matière de forêts et d'arbres. Il convient d'envisager la possibilité d'élargir ce groupe pour $\mathrm{y}$ inclure au moins un expert forestier et/ou de proposer des auditions de spécialistes et de parties prenantes du secteur forestier. Il est également important de s'engager avec les autres domaines de travail d'intérêt et de se tenir au courant de leurs activités afin de proposer des contributions 
en temps utile. Ces contributions doivent être concises, fondées sur des preuves et elles doivent également intégrer autant que possible des données économiques. Le fait que ces contributions soient informées par un large réseau d'experts et d'acteurs est susceptible de leur donner plus de poids.

Étant donné l'importance de l'aménagement du territoire pour la foresterie et ses liens avec d'autres questions qui sont souvent traitées au niveau local, en particulier la gestion des ressources naturelles, les terres, l'eau et la biodiversité, une présence active dans les mécanismes infranationaux de préparation et de mise en œuvre est essentielle, notamment pour préparer la mise en œuvre dans une perspective paysagère.

La réalisation des principaux objectifs mondiaux de lutte contre la pauvreté et pour la durabilité sera grandement facilitée par le renforcement des cadres juridiques qui reconnaissent et garantissent les droits des communautés locales et des petits exploitants à accéder aux forêts et aux arbres. L'accès aux terres, aux ressources et aux investissements dans et autour des forêts peut conduire les femmes, les jeunes et les autres entrepreneurs ruraux à devenir des agents de changement dans la transformation vers un monde durable. Un environnement positif et favorable est fondamental pour attirer le secteur privé vers des activités propices à la durabilité. L'engagement des parties prenantes et la volonté de bonne gouvernance sont tous deux essentiels pour une mise en œuvre efficace des politiques. Enfin, l'investissement dans un suivi efficace aux niveaux national et infranational fournira aux pays des informations vitales sur les groupes de personnes ou les zones du pays sur lesquels il convient de se concentrer.

\section{FIGURE 4.}

\section{Flux possibles pour l'intégration des secteurs agricoles dans les PNA (à adapter)}

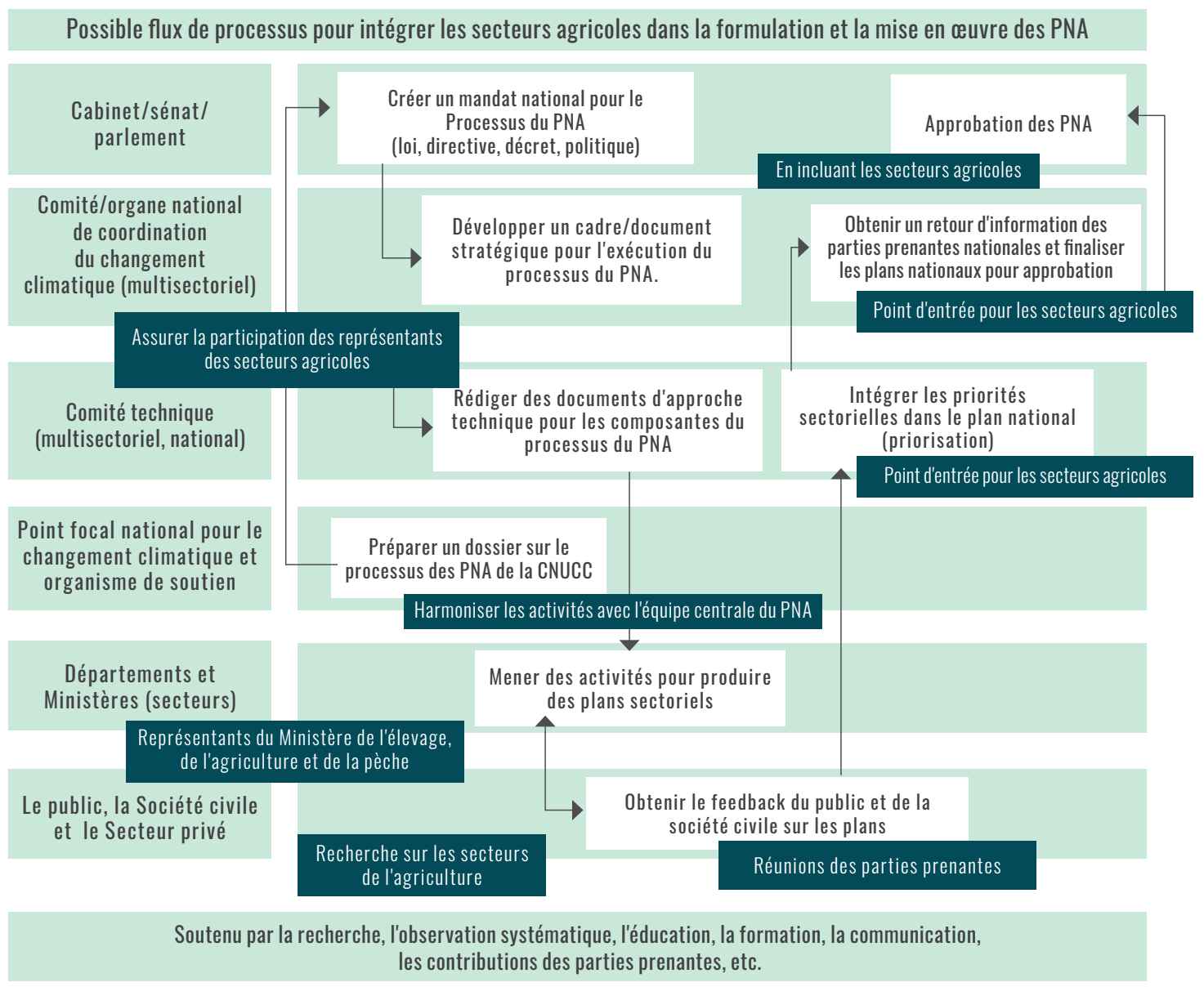

Source: Adopté par les directives techniques pour le processus des Plans d'Adaptation Nationaux du UNFCCC (UNFCCC, 2012) 


\section{- Préparer le terrain et combler les lacunes}

\author{
L'objectif de \\ cette section est \\ de décrire les \\ principales étapes \\ du premier élément \\ du processus $d u$ \\ PNA, à savoir \\ préparer le travail \\ et identifier les \\ lacunes. Cette \\ section correspond, \\ avec la précédente, \\ à l'élément $A$ \\ des directives \\ techniques du PNA.
}

\subsection{Faire le point sur les connaissances et les politiques existantes dans le secteur forestier et identifier les lacunes}

L'objectif de cette étape est de rassembler tous les éléments utiles permettant de soutenir l'intégration de la foresterie et de l'agroforesterie dans le PNA et d'identifier les lacunes en matière de connaissances. Cette étape comprend:

- la collecte de données et d'informations pour évaluer l'importance de la foresterie et de l'agroforesterie (y compris les cultures arboricoles), en tant que secteur économique, en tant que moyen d'existence pour une partie de la population et en tant que fournisseur d'autres services écosystémiques;

- un examen des institutions, des politiques et des mesures qui ont des répercussions sur la foresterie et l'agroforesterie, dans les domaines de la Foresterie, de l'agriculture, de la pêche ainsi que dans d'autres secteurs connexes tels que la gestion de l'eau, l'aménagement du territoire et l'énergie;

- les principaux points de la CND relatifs à la contribution des forêts et des arbres à l'atténuation et à l'adaptation, y compris la restauration, le boisement, la réduction de la déforestation;

- les autres engagements connexes, tels que les engagements de restauration;

- un examen des évaluations existantes de la vulnérabilité et des risques pour les forêts et les arbres;

- l'identification des stratégies, politiques, plans et investissements qui auront une influence sur le secteur forestier, y compris par exemple les stratégies d'aménagement du territoire, l'agriculture et les objectifs de sécurité alimentaire;

- les perspectives d'évolution, y compris une projection des vecteurs potentiels de changement;

- l'identification des principales lacunes en matière de connaissances;

- l'identification des lacunes et des capacités institutionnelles.

Cette étape devrait avoir une portée nationale et, au besoin, se concentrer sur des zones géographiques, des forêts, des productions 
et des chaînes de valeur spécifiques. Des informations générales sur les évaluations existantes de la vulnérabilité et des risques peuvent être extraites des documents transmis à la CCNUCC, notamment les communications nationales, les CND et les PANA. Cette vue d'ensemble peut être complétée en utilisant les évaluations du GIEC, en particulier les sections régionales et sectorielles - principalement celles qui portent sur les forêts - et la littérature scientifique à laquelle elles font référence. Il est également probable que diverses évaluations de la vulnérabilité et des risques ont déjà été réalisées dans le pays, y compris au niveau local. Le cadre méthodologique de la FAO pour les évaluations de la vulnérabilité des forêts et des populations dépendantes des forêts (Meybeck, Rose et Gitz, 2019) fournit des exemples et des références.

Un élément important est la cartographie des instruments publics nationaux et/ou infranationaux qui orientent la gestion des forêts. Une grande variété de ces instruments est potentiellement en place et souvent utilisés de manière combinée, il s'agit des lois et règlements, de la gestion directe ou déléguée des forêts publiques, des incitations, y compris les régimes fiscaux, du soutien aux instruments fondés sur le marché. Les législations nationales définissent ce qu'est une forêt, les zones qui sont considérées comme des forêts permanentes, et les droits fonciers sur les forêts et les arbres. Elles peuvent également contenir des dispositions concernant la gouvernance et la gestion des forêts, à différents degrés de détail. Des règles différentes s'appliquent souvent à différentes catégories de forêts, par exemple les domaines forestiers permanents, les forêts communautaires, les terres forestières privées, ou aux forêts destinées à différentes fonctions, comme la forêt de production ou la forêt de protection. Les lois nationales peuvent contenir des dispositions spécifiques pour les zones protégées ou pour le statut spécifique accordé à certaines forêts en raison de leur situation ou de leur fonction. Un tel statut s'accompagne généralement de règles de gestion spécifiques qui influencent la vulnérabilité de la forêt ellemême ainsi que des personnes qui en dépendent. Ces règles peuvent être déterminées au niveau national ou être partiellement ou totalement déléguées aux autorités infranationales ou à des entités spécifiques. Ces politiques et institutions constituent une base essentielle sur laquelle établir des mesures d'adaptation (voir section 6.5 Comment rendre l'environnement plus favorable).
De nombreux pays ont fixé des objectifs très ambitieux en termes de conservation, de restauration et/ou de boisement des forêts dans leur CND, et ils ont également pris des engagements en matière de restauration et définis des plans de développement. Ces objectifs doivent être pris en compte lors de l'élaboration du PNA. Premièrement, parce que ces projets doivent être réalisés de manière qu'ils soient résistants au changement climatique. Deuxièmement, parce que la manière dont ils sont menés peut également contribuer à l'adaptation d'autres secteurs. Par exemple, l'engagement de planter un certain nombre d'hectares de forêt pourrait apporter des avantages en matière d'adaptation si ces forêts sont situées dans des zones où elles peuvent réduire l'impact des inondations, de l'érosion ou de l'élévation du niveau de la mer.

Un autre élément essentiel du processus des PNA, étant donné leur objectif d'intégrer l'adaptation dans les politiques existantes, est de comprendre les nombreuses politiques qui ont une influence sur les forêts et les arbres, ainsi que leur contribution potentielle à l'adaptation d'autres secteurs, en particulier l'aménagement du territoire, la biodiversité, l'agriculture, l'eau, l'énergie et les établissements humains.

Enfin, à ce stade préliminaire, il est également particulièrement utile d'identifier les principales lacunes dans les connaissances relatives au secteur forestier afin de pouvoir mener des études complémentaires. Parmi les lacunes les plus fréquentes en matière de connaissances, il convient de mentionner les suivantes:

- les critères d'évaluation de l'état sanitaire des forêts;

- la composition et les conditions sanitaires des forêts;

- l'étendue et la composition des autres systèmes arboricoles, y compris l'agroforesterie;

- la valeur du bois et en particulier celle des produits forestiers non ligneux;

- la contribution des forêts aux moyens d'existence locaux, y compris l'autoconsommation et l'économie informelle, avec une répartition par groupes sociaux et par sexe;

- le nombre d'emplois et la valeur ajoutée générés par la transformation et la distribution de produits forestiers ligneux et non ligneux;

- les estimations de la valeur économique créée par les services écosystémiques générés par les forêts et les arbres; 
- la vulnérabilité des forêts et/ou des espèces d'importance majeure pour des raisons environnementales, économiques, sociales ou culturelles et religieuses, ainsi que celle des populations vulnérables dépendantes de la forêt.

À ce stade, des études complémentaires visant à combler les lacunes les plus urgentes en matière de connaissances et à recueillir des informations et des données devraient être lancées, en fonction des ressources disponibles et du calendrier. Il est également important de rester conscient de ces lacunes dans les connaissances et de leur pertinence pour le PNA, de sorte que lorsque de nouvelles études sont lancées ou que les systèmes de collecte de données sur les forêts sont étendus ou améliorés, ces informations puissent être efficacement canalisées dans le processus du PNA.

Ce bilan prend également en compte les analyses menées sur d'autres secteurs et domaines vulnérables pour lesquels les forêts et les arbres peuvent contribuer à accroître la résilience et établis des liens avec ces secteurs.

\section{2 Évaluer les besoins en capacités et développer les capacités pour la planification de l'adaptation des forêts et des arbres}

Cette étape se concentre sur le développement de la capacité institutionnelle et technique pour l'adaptation des forêts et des arbres, et sur la sensibilisation au potentiel des forêts et des arbres à contribuer à l'adaptation, y compris par la communication et la formation. Son objectif est d'identifier les principaux domaines dans lesquels les lacunes et les faiblesses des capacités doivent être identifiées et examinées pour ensuite proposer des moyens potentiels pour combler ces lacunes, ainsi que des exemples d'activités de communication et de formation s'appuyant sur les expériences des pays. Ces évaluations des capacités doivent être menées non seulement au niveau national, mais aussi à divers niveaux sectoriels et infranationaux tels que: les administrations locales, la société civile locale, les universités locales et autres diffuseurs de connaissances, car cela peut constituer un obstacle majeur à une planification, un développement et une mise en œuvre efficaces des programmes.

L'Institut des Nations unies pour la formation et la recherche (UNITAR) a conçu un cadre pour évaluer la capacité institutionnelle de préparation des PNA (MacKay et al., 2015). L'encadré 4 ci-dessous a adapté le cadre d'évaluation au secteur forestier. Ce cadre peut aider à déterminer le niveau des capacités et des compétences existantes pour s'engager dans des processus plus larges de PNA, pour formuler et mettre en œuvre la planification de l'adaptation au changement climatique dans le secteur et pour financer et contrôler l'efficacité des mesures d'adaptation. Il peut également fournir un moyen efficace de collecter et d'analyser les informations sur le profil de compétences existant d'un pays et d'identifier les lacunes et les stratégies de renforcement des capacités (MacKay et al., 2015) comme le montrent les exemples fournis dans l'encadré 4.

\section{ENCADRÉ 4.}

Exemples de questions pour évaluer la capacité institutionnelle du secteur forestier à planifier l'adaptation au changement climatique

\section{État de préparation du secteur des forêts, des arbres et de l'agroforesterie à l'engagement intersectoriel dans le cadre du PNA}

Existe-t-il un organe de planification/coordination national et intersectoriel du PNA (par exemple, une équipe spéciale, un groupe de travail)?

Existe-t-il une obligation (ou une invitation) pour le secteur forestier à prendre part à la planification et à la mise en œuvre de l'adaptation nationale et intersectorielle (par exemple, l'élaboration du PNA)? 
Le secteur forestier est-il représenté dans l'organe transsectoriel du PNA au niveau national ou infranational?

Le processus et le calendrier d'élaboration et de mise en œuvre du PNA sont-ils clairs pour le secteur forestier?

Existe-t-il une stratégie d'engagement intersectoriel du secteur forestier dans le PNA?

\section{État de préparation du secteur forestier aux politiques du PNA}

Existe-t-il une compréhension claire/un accord sur les impacts/risques du changement climatique pour les forêts et les systèmes arboricoles, les secteurs, les communautés?

Existe-t-il une exigence/demande de développement de plans d'adaptation sectoriels/PNA?

Les évaluations de l'impact et de la vulnérabilité dans la foresterie sont-elles requises par le cadre politique / juridique pour utilisation dans la planification de l'adaptation?

- Existe-t-il un plan d'adaptation des forêts au changement climatique?

L'adaptation est-elle intégrée dans les politiques, stratégies et plans relatifs aux forêts, aux arbres et à l'agroforesterie?

- dans quelle mesure? (par exemple FAIBLE: une brève mention du changement climatique, à ÉLEVÉ: spécification des vulnérabilités au changement climatique et des actions d'adaptation /stratégie / plan d'adaptation pour les forêts).

Les plans et politiques de DRM pertinents sont-ils intégrés dans les plans de développement forestier ou d'adaptation?

\section{État de préparation organisationnelle du secteur forestier au PNA}

Existe-t-il une unité officielle chargée du changement climatique (équipe spéciale, groupe de travail, etc.) au sein de l'institution en charge de la foresterie?

Cette unité de gestion du changement climatique a-t-elle reçu un mandat clair pour planifier et coordonner l'adaptation dans le secteur?

L'unité de gestion du changement climatique bénéficie-t-elle d'une légitimité en haut lieu (par exemple, l'approbation du ministrere des forêts) pour ses actions?

Existe-t-il un point focal pour le changement climatique au sein du Ministère/ département des forêts (aux niveaux national et provincial)?

- Les responsabilités du point focal concernant le changement climatique et la ligne d'intervention sont-elles bien définies?

- Dans quelle mesure le travail du point focal est-il lié aux autres secteurs qui affectent et/ou sont affectés par la foresterie (par exemple, les cultures et le bétail, l'aménagement du territoire, les eaux intérieures, l'énergie, ...)?

- Le point focal a-t-il des interactions régulières avec l'organe de gestion du PNA pour faciliter les discussions interministérielles?

La visibilité actuelle du changement climatique dans la structure organisationnelle des institutions en charge de la foresterie est-elle suffisante? Quelle est la visibilité du rôle et des actions du point focal?

La visibilité du changement climatique dans le travail du Ministère des forêts doitelle être renforcée?

(par exemple, augmenter le nombre d'employés, accorder des promotions, changer de superieur hierarchique). 


\section{État de préparation technique du secteur forestier au PNA}

Des données sont-elles régulièrement collectées sur les paramètres environnementaux, météorologiques et climatiques et mises à disposition pour être utilisées dans la planification/gestion?

- Existe-t-il un système d'information climatique et environnementale/un système de prévision saisonnière mis à la disposition des gestionnaires des forêts de manière continue?

- Existe-t-il un système d'information bio-climatique qui informe, par exemple, des changements dans l'abondance et la distribution des espèces, et qui est disponible de façon continue pour les gestionnaires des forêts et les parties prenantes?

Les impacts/risques du changement climatique pour les forêts, les arbres et l'agroforesterie, ainsi que pour les personnes qui en dépendent, ont-ils été évalués?

- Existe-t-il des évaluations de la vulnérabilité des forêts et des populations qui en dépendent?

- Des outils d'analyse tels que l'évaluation de la vulnérabilité et des risques, l'analyse de scénarios, l'analyse coûts-bénéfices sont-ils utilisés pour comprendre les besoins d'adaptation du secteur?

- Ces analyses prennent-elles en compte les sous-secteurs, la diversité des acteurs (petite échelle, grande échelle), les zones géographiques (provinces/districts, communauté, national) et les écosystèmes?

Les résultats de ces analyses sont-ils pris en compte dans la planification et l'élaboration des politiques?

Des options d'adaptation appropriées ont-elles été identifiées pour les forêts et les systèmes arboricoles?

\section{État de préparation du secteur de la pêche et de l'aquaculture en matière de financement, de suivi et de communication dans le cadre du PNA}

Existe-t-il entre le secteur forestier et les autres secteurs de l'économie un cadre commun de financement de l'adaptation au changement climatique?

Les coûts de l'adaptation pour le secteur ont-ils été évalués?

- Qu'est-ce qui ressort de leur comparaison avec (i) la valeur du secteur, (ii) le budget de développement du secteur (iii) le budget alloué par le secteur á la question de l'adaptation?

Un budget est-il clairement affecté aux évaluations de la vulnérabilité au changement climatique et aux activités d'adaptation dans le secteur forestier?

- Ce budget est-il facilement accessible?

Un système national de suivi et d'évaluation de l'adaptation est-il en place pour le secteur afin de surveiller la réduction de la vulnérabilité/la capacité d'adaptation et l'efficacité des actions d'adaptation?

- Les données sur l'adaptation sont-elles régulièrement collectées à des fins de suivi et d'évaluation?

Existe-t-il une stratégie de communication et un budget pour le département du Ministère des forêts en charge du travail d'adaptation? 
ENCADRÉ 5.

\section{Évaluation des besoins en capacités de l'autorité nationale de gestion de la sécheresse du Kenya dans le cadre du PNA}

Dans le cadre du volet Agriculture du Programme national d'Adaptation (PNA) du Kenya, une évaluation complète a été réalisée sur la capacité de l'Autorité nationale de Gestion de la Sécheresse du Kenya (NDMA) à prendre en charge la planification et la mise en œuvre des actions d'adaptation et des mesures prioritaires concernant le secteur agricole. La mission de la NDMA est d'assurer la direction et la coordination des efforts du Kenya dans la gestion des risques de sécheresse et le renforcement de l'adaptation. Son rôle, ses fonctions, sa structure de gestion et ses dispositions institutionnelles en matière de changement climatique ont été passés au crible afin d'affiner la portée de l'évaluation des capacités. Celle-ci s'est intéressée à la capacité de la NDMA en matière de fonction normative et politique, de connaissances, de partenariat et de capacité de mise en œuvre pour soutenir les actions d'adaptation à court, moyen et long terme, telles que décrites dans le plan d'action sur le changement climatique et le PNA du Kenya.

L'évaluation de chaque capacité fonctionnelle a porté sur l'environnement favorable, l'organisation et les capacités individuelles. Une note de 1 à 5 a été attribuée (1 = aucune; 2 = faible; 3 = modérée; 4 = élevée; et 5 = pleine capacité). Un résumé des résultats des évaluations quantitatives est présenté dans la figure ci-dessous. Les réponses ont été recueillies auprès du personnel clé grâce à l'utilisation combinée d'entretiens avec le personnel et d'un questionnaire d'auto-évaluation participatif afin de renforcer l'appropriation des résultats.

Les conclusions de l'évaluation ont révélé que la NDMA a: (i) des capacités fonctionnelles de niveau modéré à élevé, malgré certaines limitations spécifiques concernant les arrangements institutionnels visant à répondre aux priorités d'adaptation et de réduction des risques de catastrophe (DRR) et à mettre en œuvre le mandat de la NDMA et (ii) des lacunes dans sa capacité organisationnelle et individuelle à renforcer ses fonctions et son efficacité. La création d'une unité sur le changement climatique au sein du Ministère de la décentralisation et de la planification (MoDP) était l'une des principales recommandations de l'évaluation. Les conclusions ont servi de base à l'élaboration d'une stratégie de développement des capacités et d'un plan d'action pour la NDMA.

\section{Analyse sommaire de l'évaluation des besoins en capacités au sein de l'Autorité nationale de gestion de la sécheresse du Kenya}

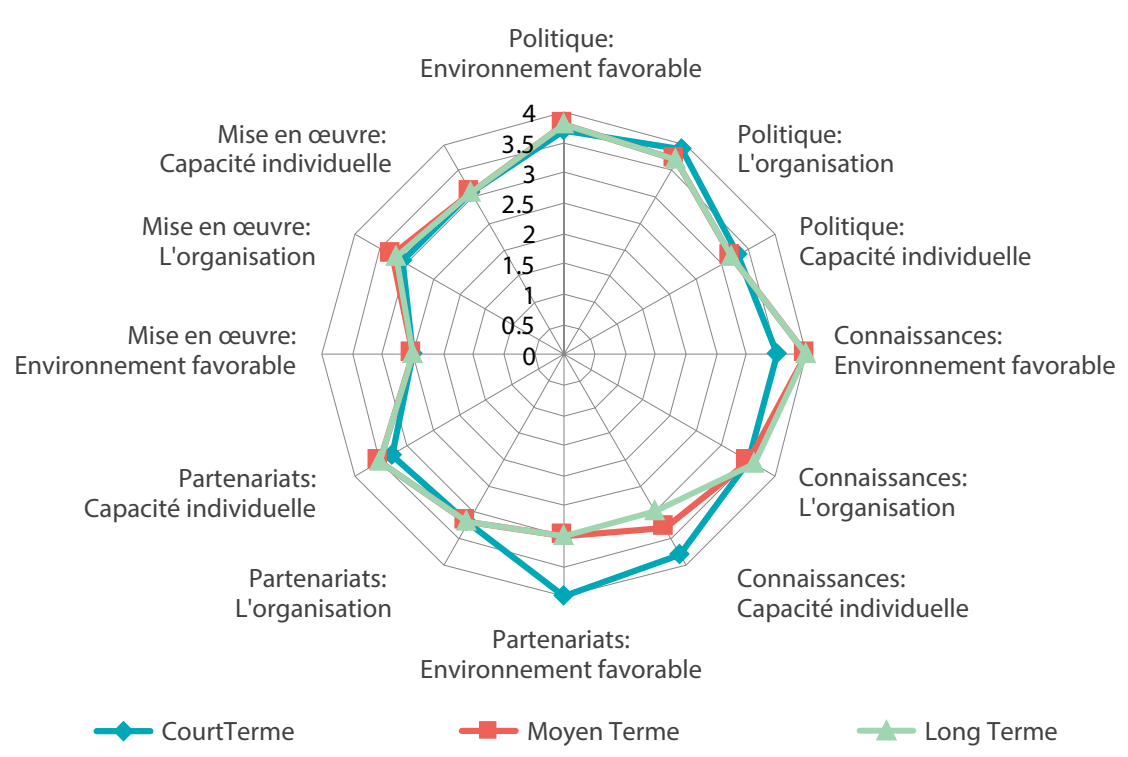


Un autre aspect important de la recherche est de savoir dans quelle mesure le secteur forestier a été impliqué dans la planification et les efforts d'adaptation au climat et comment cela peut être capitalisé.

Les questions suivantes peuvent étayer cette analyse:

\section{Le pays dispose-t-il d'un PANA ou d'un autre document/d'une autre stratégie national/e de planification de l'adaptation's?}

- Si oui, cela inclut-il la foresterie?

- Comment le secteur a-t-il été inclus dans son élaboration?

- S'il n'a pas été inclus, à quelles contraintes s'est-il heurté?

- Quels étaient les principaux acteurs non gouvernementaux impliqués dans ce processus (institutions de recherche, secteur privé, $\mathrm{ONG})$ ?

- Quels sont les résultats du PANA en ce qui concerne l'adaptation des forêts et des systèmes arboricoles au changement climatique?

- Quel est l'impact du plan d'adaptation existant sur le développement du secteur forestier?

- Existe-t-il des exemples de plans ou de stratégies infranationaux et comment le secteur forestier est-il couvert?

\section{Le secteur forestier est-il officiellement} engagé dans le processus de formulation et de mise en œuvre du PNA du pays?

- Si oui, comment et dans quelle mesure? (mécanismes de mobilisation, contributions à ce jour, etc.)

- Quelles sont les parties prenantes impliquées (recherche, secteur privé, ONG)?

- Comment les communautés locales, les petits exploitants sont-ils associés?

- Si ce n'est pas le cas, comment y remédier?

$\checkmark$ Le secteur a-t-il pris part à des discussions, plans ou stratégies sur le changement climatique

- Si oui, qu'en est-il ressorti et comment peuton aller plus loin? Si non, pourquoi?
Source: FAO, 2018b

- Des mécanismes de consultation sont-ils en place?

- L'adaptation au changement climatique dans le secteur forestier est-elle spécifiquement mentionnée dans l'un des documents stratégiques du pays?

\section{Les politiques, stratégies et plans de} développement forestier passés et actuels tiennent-ils compte des facteurs de changement climatique?

- Comment le pfn du pays prend-t-il en compte la question du changement climatique?

- Savons-nous ce qui est nécessaire, de quoi s'agit-il?

- Comment ces programmes affectent-ils le secteur?

- Quelle est leur pertinence?

- Pourraient-ils être intégrés dans le PNA tels quels ou après révision ou validation?

- Si non, pourquoi? et pourrait-on (ou devraiton) les modifier?

Les objectifs de cette étape sont les suivants:

- Identifier les points d'entrée, y compris le pfn du pays pour engager les forêts, les arbres et l'agroforesterie dans le PNA.

- Comprendre les possibilités existantes pour la foresterie de s'engager dans le processus de formulation et de mise en œuvre du PNA.

- Identifier les lacunes politiques et organisationnelles à combler pour soutenir la planification de l'adaptation au sein du secteur forestier, notamment par le biais du pfn, et faciliter la participation du secteur à une planification plus large et intersectorielle de l'adaptation au changement climatique.

- Identifier un point focal, une unité ou une équipe spéciale ou tout autre mécanisme ou organe officiellement reconnu chargé de diriger l'intégration des forêts, des arbres et de l'agroforesterie au cours du processus de formulation et de mise en œuvre du PNA.

- Identifier les lacunes en matière de compétences et définir une stratégie pour les combler.

5 Il peut s'agir d'une stratégie de lutte contre le changement climatique, de mécanismes de mise en œuvre des CDN, d'un plan d'action ou d'une politique de lutte contre le changement climatique, etc. et peut inclure des priorités d'adaptation et d'atténuation. 


\section{3 Évaluer et identifier les liens entre les besoins d'adaptation et les objectifs de développement}

L'objectif de cette étape, basée sur la section 5.1, est d'évaluer comment les objectifs d'adaptation pourraient être liés aux objectifs de développement. Cette étape comprend également l'examen des stratégies et des plans de développement dans une optique d'adaptation afin d'évaluer si et dans quelle mesure ils intègrent les préoccupations d'adaptation, ou comment ils pourraient être modifiés pour mieux intégrer l'adaptation.

Parmi les documents clés à examiner pour cette étape, figurent:

- les documents stratégiques nationaux de réduction de la pauvreté, les objectifs nationaux de développement à moyen et long terme (par exemple, les objectifs du Millénaire pour le développement);

- les plans nationaux de développement et/ ou les stratégies économiques, les politiques, stratégies et plans sectoriels;

- les politiques et plans de préparation aux catastrophes et de réduction des risques;

- les plans de coopération multi et bilatéraux;

- les stratégies et programmes d'aide au développement, notamment avec les organisations internationales, les banques de développement internationales et régionales, ainsi que d'autres donateurs.

Un tel examen devrait être effectué en tenant compte de la nécessité de revoir et d'actualiser également ce type de documents afin d'intégrer l'orientation fournie par l'Agenda 2030 pour le Développement durable.

L'Agenda 2030 pour le Développement durable et ses 17 Objectifs de Développement Durable (ODD) sont devenus le cadre central pour guider les politiques de développement dans les pays du monde entier. Il a été délibérément conçu pour intégrer des objectifs «interdépendants et indivisibles». Il a été noté qu'un tiers des ODD contribuent à l'adaptation au changement climatique et à son atténuation, avec le plus grand potentiel pour générer des synergies dans l'agriculture, l'eau, les déchets alimentaires et les écosystèmes marins et forestiers (WRI, 2018).
Le rapport sur la Situation des forêts du monde 2018 (FAO, 2018a) fournit une analyse détaillée visant à saisir la contribution des forêts et des arbres à 28 objectifs relatifs à dix ODD. Les principales conclusions peuvent aider à orienter la réflexion pour renforcer les synergies et minimiser les compromis entre les objectifs de développement et les objectifs d'adaptation. Les forêts et les arbres apportent une contribution vitale à la fois aux populations et à la planète, en renforçant les moyens d'existence, en fournissant de l'air et de l'eau propres, en conservant la biodiversité et en réagissant au changement climatique. La gestion des forêts influence la quantité et la qualité de l'eau, essentielle à la santé et à la vie des populations rurales et urbaines.

Ces liens entre les forêts et d'autres secteurs devraient être soulignés et explorés, en tenant compte de leur influence sur la réalisation des ODD, mais aussi sur la façon dont les actions menées dans d'autres secteurs pour l'atteinte d'autres ODD peuvent (ou non) faciliter l'adaptation des forêts. Les actions visant à atteindre certains objectifs de développement peuvent créer des synergies pour l'adaptation des forêts et des populations tributaires des forêts et renforcer le rôle des forêts dans l'adaptation de manière plus générale.

L'un des grands défis de notre époque est de savoir comment augmenter la production agricole et améliorer la sécurité alimentaire sans réduire la superficie des forêts. L'amélioration de l'efficacité des ressources, la production durable et les nouvelles technologies (ODD 12) et l'augmentation de la productivité agricole (ODD 2) peuvent réduire les pressions exercées sur les forêts. Du point de vue de la réduction de la pauvreté (ODD 1), les actions doivent tenir compte des forêts et des populations qui en dépendent, car les personnes les plus démunies se trouvent souvent dans des zones situées à l'intérieur et autour des forêts.

L'énergie (ODD 7) est un autre exemple clé, car de nombreux ménages dans les pays en développement dépendent de la bioénergie traditionnelle provenant des forêts pour la cuisine et le chauffage. La modernisation du secteur traditionnel de l'énergie du bois peut améliorer les moyens d'existence, créer des chaînes de valeur durables et libérer des ressources pour les investissements dans la gestion durable des forêts. En fait, la transition vers des alternatives énergétiques modernes et des technologies à efficacité améliorée peut réduire la dépendance 
au bois en tant que combustible, tandis que l'efficacité peut être améliorée et les pressions qui en résultent sur les ressources forestières minimisées.

Il existe des preuves quantitatives montrant que les forêts et les arbres contribuent à la réalisation des ODD liés aux moyens d'existence et à la sécurité alimentaire de nombreux pauvres en milieu rural, ainsi qu'à l'accès à une énergie abordable, à une croissance économique et à un emploi durable (dans le secteur formel), à une consommation et à une production durable, à l'atténuation du changement climatique et également à la gestion durable des forêts. En outre, des données qualitatives suggèrent que les forêts et les arbres apportent une contribution importante aux ODD dans le secteur informel, l'agroforesterie, les possibilités d'autonomisation des femmes, la gestion durable de l'eau, le tourisme, les villes durables, l'adaptation au changement climatique et la lutte contre la dégradation des terres et la perte de biodiversité.

Toutefois, les relations entre les forêts et les ODD doivent également reconnaître la réalité des compromis existants (par exemple, Katila et al., 2019) entre l'ensemble des objectifs de développement durable et les forêts. Ces compromis doivent être gérés avec l'objectif d'éviter de compromettre la capacité des forêts et des populations qui en dépendent à s'adapter au changement climatique. Par exemple, l'expansion des cultures agricoles pour l'alimentation ou l'énergie (ODD 1 et 7) peut concurrencer les forêts dans de nombreux endroits, et l'ODD 9 (Industrie, innovation et infrastructure) peut encourager le développement des infrastructures et d'autres activités aux dépens de la zone forestière.

Comme mentionné dans la section 2.1, la plupart des pays qui ont intégré une composante d'adaptation dans leur CDN ont défini une vision à long terme qui les guide. L'agriculture joue souvent un rôle de premier plan en raison de son potentiel tant pour le développement durable que pour les actions de lutte contre le changement climatique. Son intégration dans les PNA offre des possibilités considérables d'apporter une contribution aux ODD (Juergenliemk-Avagyan et al., 2017) grâce aux multiples retombées positives de l'adaptation dans les secteurs de l'agriculture et de l'utilisation des terres (FAO, 2020b). Une analyse des secteurs de l'agriculture dans les CPDNs (Strohmaier et al., 2016) a noté que les pays perçoivent les synergies d'adaptation et d'atténuation et les avantages qui y sont associés comme des éléments importants de leurs stratégies de développement à long terme. Une analyse des CND des pays d'Europe du Sud-Est et d'Asie centrale (FAO, 2019) souligne que les actions menées dans le secteur forestier sont particulièrement susceptibles de générer des retombées positives en termes d'adaptation et d'atténuation et que la convergence croissante entre la mise en œuvre des objectifs relatifs au changement climatique et des ODD offre de grandes possibilités d'accélérer les progrès vers ces deux objectifs. L'analyse régionale des CDN pour l'Asie a tiré des conclusions similaires (FAO, 2020c). La plupart des mesures d'atténuation et d'adaptation dans les secteurs agricoles sont concentrées dans le secteur forestier et soulignent le caractère unique de sa capacité à fournir des biens mondiaux (stabilisation du climat) et à soutenir les moyens d'existence locaux. Environ 60 pour cent des pays d'Asie reconnaissent explicitement les avantages connexes, soit entre l'adaptation et l'atténuation, soit avec les ODD dans les secteurs agricoles, en particulier dans le secteur forestier.

La nécessité de poursuivre des objectifs multiples n'est pas nouvelle pour le secteur forestier. L'Assemblée générale des Nations unies (UNGA, 2008) reconnaît «que la gestion durable des forêts (GDF), en tant que concept dynamique et évolutif, vise à maintenir et à renforcer les valeurs économiques, sociales et environnementales de tous les types de forêts au profit des générations présentes et futures». L'objectif même de la GDF est de renforcer les synergies et de gérer les compromis entre les objectifs, y compris dans les particularités mêmes de la gestion et des pratiques. Une telle entreprise peut souvent être mieux menée au niveau du paysage (voir l'exemple de l'Indonésie, encadré 6). La planification des paysages forestiers doit prendre en compte les besoins des autres secteurs (par exemple, la production de bioénergie, la production de cultures) et intégrer ces besoins comme critères dans le choix des espèces à utiliser (par exemple, les espèces à taux de croissance élevé, les espèces de légumineuses pour augmenter la disponibilité des nutriments du sol pour les systèmes de cultures intercalaires). Ces objectifs multiples exigent d'intégrer pleinement le changement climatique dans les politiques forestières nationales (FAO, 2018e). 
ENCADRÉ 6.

\section{Extrait des contributions déterminées au niveau national de l'Indonésie}

L'approche stratégique de la CDN de l'Indonésie repose sur les principes fondamentaux suivants:

- Utiliser une approche paysagère: en reconnaissant que les efforts d'adaptation et d'atténuation du changement climatique sont par nature multisectoriels, l'Indonésie adopte une approche intégrée à l'échelle du paysage couvrant les écosystèmes terrestres, côtiers et marins.

- Mettre en évidence les meilleures pratiques existantes: en reconnaissant les progrès significatifs réalisés dans les efforts multipartites de lutte contre le changement climatique, l'Indonésie entend accroître la diversité des savoirs traditionnels ainsi que les efforts innovants d'adaptation et d'atténuation du changement climatique déployés par le gouvernement, le secteur privé et les communautés.

- Intégrer l'agenda climatique dans la planification du développement: en reconnaissant la nécessité d'intégrer le changement climatique dans le développement, l'aménagement du territoire et le processus budgétaire, l'Indonésie inclura des indicateurs clés du changement climatique dans la formulation des objectifs de son programme de développement.

- Promouvoir la résilience climatique dans les domaines de l'alimentation, de l'eau et de l'énergie: en reconnaissant l'importance de répondre aux besoins d'une population jeune et croissante en matière d'alimentation, d'eau et d'énergie, l'Indonésie améliorera sa gestion des ressources naturelles afin de renforcer la résilience au changement climatique en protégeant et en restaurant les principaux écosystèmes terrestres, côtiers et marins.

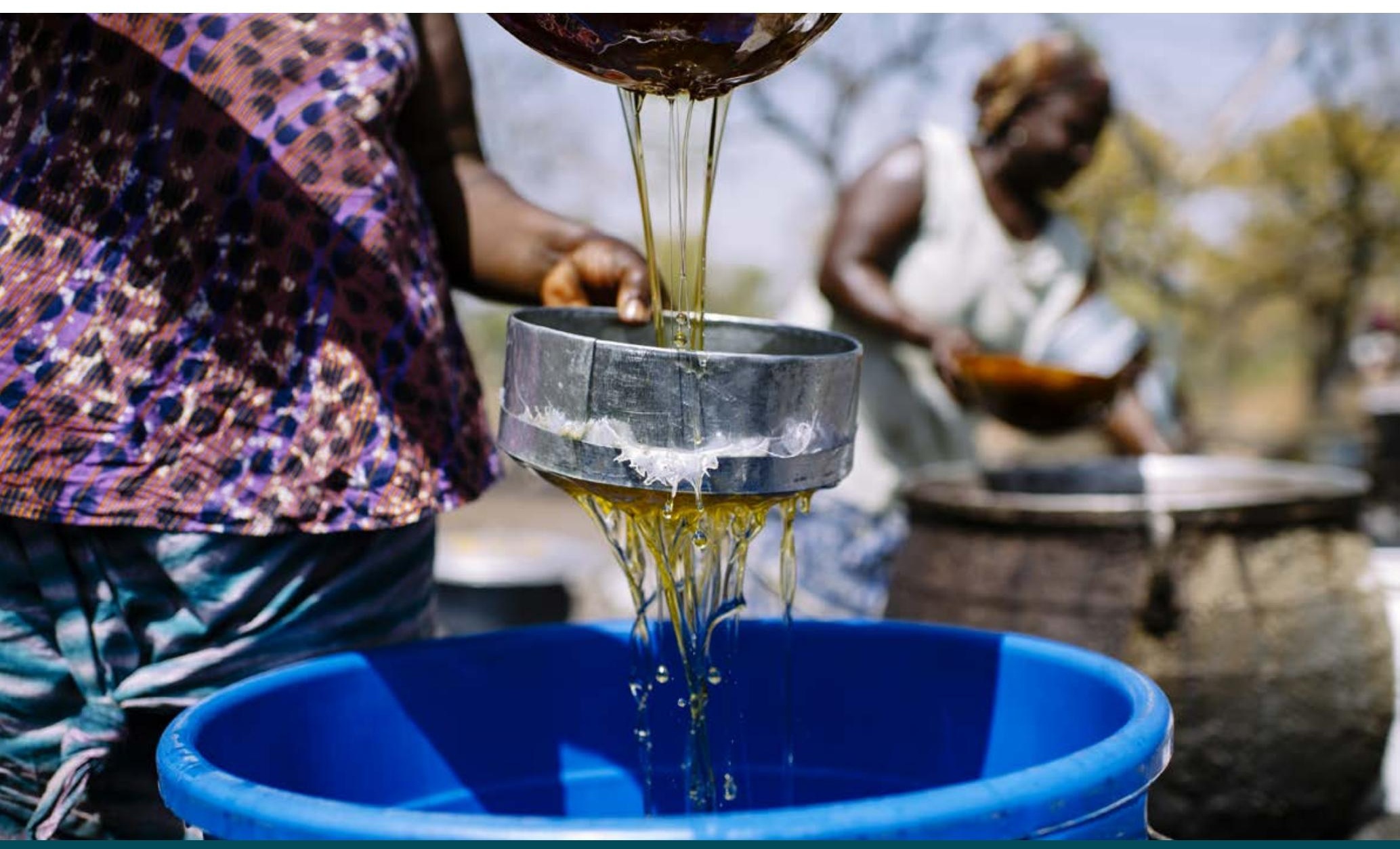




\section{- Eléments de préparation}

\author{
Cette section \\ correspond \\ à l'élément $B$ \\ des directives \\ techniques du PNA. \\ Elle traite tout \\ d'abord de l'analyse \\ des projections \\ climatiques et de \\ leurs implications \\ pour les forêts, \\ les arbres, \\ l'agroforesterie \\ et les populations \\ qui dépendantes \\ des forêts. \\ Deuxièmement, \\ elle passe en revue \\ les risques et les \\ vulnérabilités \\ potentiels, afin \\ de fournir des \\ orientations pour \\ l'identification, \\ la sélection et la \\ hiérarchisation \\ des options \\ d'adaptation.
}

BURKINA FASO, AFRIQUE

Huile de karité (beurre de karité liquéfié) filtré avant l'emballage. oCIFOR/Ollivier Girard

\subsection{Analyser les scénarios climatiques actuels et futurs en ce qui concerne les forêts et les personnes qui en dépendent}

Cette étape prend en compte les informations climatiques utilisées dans le processus du PNA, leur pertinence pour la forêt et les arbres et les principales variables, changements et dangers importants pour le secteur. Elle peut mettre l'accent sur les besoins spécifiques du secteur, par exemple des séries historiques plus longues, des projections sur une période plus longue ou désagrégées à une échelle plus locale, ou l'attention portée à des facteurs spécifiques comme les risques de vent ou d'incendie. Par la suite, elle mettra en évidence les principaux changements et dangers qui doivent être examinés pour l'évaluation de la vulnérabilité du secteur.

La première étape consiste à consulter les informations et les projections climatiques utilisées pour le processus global du PNA et à examiner si elles sont pertinentes pour les forêts et les arbres. L'un des avantages de l'utilisation de ces mêmes informations est que l'analyse relative aux forêts peut être plus facilement promue lorsqu'elle est basée sur les mêmes prémisses et scenarii. Dans le cas où des hypothèses et des paramètres différents sont utilisés, ils doivent être expliqués et justifiés, par exemple, par une situation spécifique ou par la sensibilité spécifique de la forêt ou de certains arbres importants (par exemple le café, le cacao, le caoutchouc) à certains paramètres.

Dans le cas où la vulnérabilité est estimée pour les conditions climatiques actuelles, il peut être possible d'utiliser les enregistrements météorologiques pour déterminer la moyenne historique et la fourchette des valeurs climatiques considérées comme importantes, y compris l'occurrence de phénomènes météorologiques extrêmes (par exemple, Borja-Vega et De la Fuente 2013, Dasgupta et al., 2014). En plus des sources nationales, les informations historiques sur le climat peuvent être obtenues auprès de sources mondiales, telles que l'Administration océanique et atmosphérique nationale (NOAA). Lorsqu'elles ne sont pas 
disponibles pour l'endroit précis en raison du manque de stations météorologiques par exemple, des approximations telles que des enregistrements d'une station comparable ou des moyennes à des échelles plus larges peuvent être utilisées. Toutefois, l'utilisation de ces méthodes doit tenir compte des spécificités topographiques.

Ces informations peuvent être complétées ou, si nécessaire, remplacées par des observations d'événements climatiques (par exemple, Bele et al., 2013; Dazé et al., 2009; Obeng et al., 2011; Parkins et MacKendrick, 2007). Dans de tels cas, la précision des variables quantitatives peut être moindre. D'autre part, l'utilisation de ces observations pourrait faciliter la compréhension des liens entre les phénomènes observés et les impacts concrets sur les forêts et les moyens d'existence. Dans le cas où l'on utilise à la fois des instruments d'enregistrement et des observations personnelles, il convient de prêter attention à la pondération relative des souvenirs des personnes touchées par rapport aux enregistrements du climat et des événements extrêmes déterminés par les instruments des stations météorologiques (Dasgupta et al., 2014).

Il peut être utile de mobiliser différents scénarii et méthodes ou une série de résultats afin de décrire une série de scénarii possibles. Par exemple, la méthode des futurs climatiques représentatifs (Whetton et al., 2012) vise à identifier un petit ensemble de scénarii possibles qui sont différenciés en fonction des principales variables d'intérêt et de leur probabilité. Dans la gamme des scénarii possibles dérivés des projections, on peut choisir un climat plus chaud et plus sec ou un climat plus variable et plus venteux, avec identification de leur probabilité, ainsi que du climat le plus probable. Cela permet aux parties prenantes de se préparer à des scénarios contrastés, à la fois les plus probables et les pires, ceci en fonction des variables d'intérêt pour un système spécifique.

\subsection{Identifier et évaluer les vulnérabilités des forêts, des arbres et des personnes qui dépendent des forêts au changement climatiquee}

Cette section s'appuie sur la méthodologie du Cadre pour les évaluations de la vulnérabilité des forêts et des populations qui en dépendent au changement climatique (Meybeck, Rose et Gitz, 2019), ce cadre pouvant être adaptée afin de donner une perspective nationale, complétée par des évaluations plus détaillées des zones et/ou productions prioritaires.

L'objectif est de couvrir trois domaines principaux:

- les vulnérabilités des forêts et des arbres;

- la vulnérabilité des paysages, des secteurs économiques qui sont liés aux forêts et aux arbres ou qui en dépendent;

- la vulnérabilité des personnes qui dépendent de ces secteurs et de ces paysages.

Une évaluation des vulnérabilités doit partir du bilan du secteur et de son importance dans le pays, ainsi que de ses liens avec d'autres secteurs (voir section 5.1), ce qui permet d'identifier les domaines les plus importants à examiner. Par exemple, il peut s'agir de souligner l'importance des cultures arboricoles de base pour l'économie et l'emploi dans le pays, ou l'importance de certaines espèces d'arbres qui jouent un rôle important dans des écosystèmes d'intérêt majeur en raison de leur extension ou de leur spécificité. Elle peut être orientée vers le classement de la vulnérabilité relative des espèces au climat ou vers l'évaluation des risques que le changement climatique altère des écosystèmes uniques ayant une importance écologique ou sociale. Elle pourrait également être axée sur les processus qui sont importants pour des raisons économiques, par exemple les taux de croissance et la régénération des arbres et des produits forestiers non ligneux d'importance commerciale. Cette identification des domaines prioritaires doit également tenir compte des résultats de l'évaluation de la vulnérabilité d'autres secteurs au changement climatique, 
et des contributions potentielles des forêts, des arbres et de l'agroforesterie (voir section suivante) pour réduire cette vulnérabilité. Par exemple, l'importance des mangroves et des forêts côtières doit être soulignée en raison de leur rôle dans la protection des habitations humaines.

Il existe diverses sources d'information permettant de cartographier la future distribution potentielle des espèces dans le cadre du changement climatique, en comparant le climat d'une zone spécifique à ce qui constitue des conditions favorables pour une espèce spécifique. Un nombre croissant d'études modélise les impacts potentiels du changement climatique pour des espèces d'intérêt dans une zone déterminée, par exemple, des espèces d'arbres alimentaires au Burkina Faso (Gaisberger et al., 2017) pour projeter des zones de production de bananes et de café au Népal (Ranjitkar et al., 2016a) ou pour soutenir la sélection d'espèces agroforestières dans la province du Yunnan, en Chine (Ranjitkar et al., 2016b). L'Atlas intitulé «Adéquation des principales espèces agroforestières d'Amérique centrale dans les climats futurs» (de Souza et al., 2017) présente des cartes d'adéquation actuelles et futures pour 54 espèces qui sont couramment utilisées comme ombrage dans les systèmes agroforestiers en Amérique centrale. Les 54 espèces d'arbres sélectionnées comprennent 24 espèces d'arbres fruitiers, 24 arbres de bois d'œuvre et six espèces utilisées pour améliorer l'état des sols.

Il est important de tenir compte des impacts indirects, des perturbations et des changements dans l'écosystème, comme les incendies, les parasites, les maladies, les perturbations du fonctionnement de l'écosystème, y compris la pollinisation et les relations entre les parasites et leurs ennemis naturels, et les changements dans la composition des espèces. La sécheresse a souvent des conséquences indirectes telles que les incendies de forêt, l'augmentation de l'incidence des parasites et des maladies profitant de la faiblesse des arbres, qui à leur tour créent de la biomasse morte et favorisent la propagation du feu; le feu ayant par la suite un impact sur la répartition des espèces. En fait, une étude a permis de modéliser les régimes d'incendie actuels et futurs et de décrire leur influence sur la végétation naturelle en Éthiopie (van Breugel, Friis, Demissew et al., 2016). Le changement climatique peut également modifier la dynamique des insectes ravageurs, comme c'est le cas pour les espèces de pyrales des pins en Europe. Les études de dendrochronologie peuvent être particulièrement utiles car elles fournissent des informations sur les conditions que les arbres existants ont connues dans le passé (Gebrekirstos et al., 2014).

Il est essentiel d'analyser les interactions potentielles des effets induits par le changement climatique avec d'autres causes de vulnérabilité et surtout d'autres pressions humaines sur les forêts. L'identification des autres menaces et changements (autres que ceux liés au changement climatique) tels que la déforestation et la dégradation des forêts et la manière dont ils affectent la forêt est fondamentale, en particulier lorsque l'évaluation est menée dans une perspective à long terme. Cette analyse doit commencer par les vulnérabilités actuelles telles que la dégradation, la surexploitation des produits forestiers non-ligneux, l'introduction d'espèces envahissantes, le manque de bonne gouvernance, la mauvaise application des lois existantes et la pression démographique, tout ceci avant de prédire comment ces facteurs pourraient évoluer à l'avenir.

Une façon de le faire est d'examiner les tendances passées et actuelles de la déforestation et de la dégradation des forêts ainsi que leurs causes et leurs moteurs. Au niveau mondial, les forêts sont de plus en plus sollicitées pour leur conservation, pour la production de bois et de produits forestiers non-ligneux avec la concurrence d'autres activités, notamment l'agriculture pour les terres. Cependant, ces demandes s'expriment différemment au niveau local. Il y a aussi des zones où l'agriculture (cultures et bétail) abandonne en fait la terre et où il y a des reboisements ou des plantations spontanés. Il existe également une tendance à une gestion plus durable des forêts dans de nombreuses régions. Cela peut être complété en étudiant les activités humaines qui ont lieu dans ou autour des forêts, leurs impacts potentiels, et leur propre tendance, stable, croissante, décroissante. Une source d'information supplémentaire peut être trouvée dans les stratégies et les plans qui pourraient avoir un impact sur la forêt, positif, comme un projet de conservation ou de gestion durable, et négatif, comme de nouvelles routes et mines. La combinaison de ces éléments permet d'identifier les orientations possibles des différents facteurs 
de vulnérabilité qui pourraient à leur tour être influencés par le changement climatique ou interagir avec ses impacts.

Les effets du changement climatique et d'autres événements sur les populations locales peuvent créer un stress supplémentaire pour les forêts et doivent donc être considérés comme une source supplémentaire de vulnérabilité. Par exemple, les installations de personnes déplacées créent une pression supplémentaire sur les forêts, notamment en raison de la demande accrue de bois de chauffage, et cette pression doit être évaluée et traitée ultérieurement pour éviter la dégradation de l'environnement (d'Annunzio et al., 2016).

Les impacts sur les forêts et les arbres ont à leur tour des répercussions sur les personnes qui en dépendent. Comme le montre l'encadré 7, la notion de personnes qui dépendent des forêts peut couvrir un grand nombre et une grande diversité de personnes et doit être examinée pour chaque situation spécifique.

\section{ENCADRÉ 7.}

\section{Les personnes qui dépendent de la forêt}

La notion de dépendance vis-à-vis de la forêt a souvent été principalement définie par la contribution économique directe aux moyens d'existence des produits forestiers, soit directement consommés, soit vendus. Fisher et al. (1997) distinguent trois types de personnes qui dépendent des forêts:

a) Les personnes qui vivent dans les forêts naturelles et aux alentours ou à la frontière des forêts, souvent en tant que chasseurs-cueilleurs ou cultivateurs itinérants, et qui sont fortement dépendantes des ressources forestières comme moyens d'existence principalement, mais pas toujours comme tel. Les cultures itinérantes contribuent largement à leur sécurité alimentaire. Les personnes appartenant à cette catégorie sont souvent des peuples indigènes ou des personnes issues de groupes ethniques minoritaires. Elles sont donc généralement en dehors du courant politique et économique dominant.

b) ) Les personnes qui vivent à proximité des forêts et qui sont généralement impliquées dans des pratiques agricoles à l'intérieur ou à l'extérieur de la forêt, et qui utilisent régulièrement les produits de la forêt (bois d'œuvre, bois de chauffage, aliments de brousse, plantes médicinales, etc.) Pour ceux qui sont plus impliqués dans l'agriculture, les compléments alimentaires provenant des forêts sont souvent d'une importance capitale pour la sécurité alimentaire et la nutrition.

c) Les personnes exerçant des activités commerciales comme la chasse, la collecte de minéraux ou les industries forestières telles que la gestion des forêts et l'exploitation forestière. Ces personnes peuvent faire partie d'une économie mixte de subsistance et de rente et dépendent de la forêt principalement comme source de revenus en espèces. Toutefois, il est important de noter que ce type d'interaction entre la population et la forêt peut exister même dans un contexte fortement monétisé: par exemple, certaines petites communautés rurales dans des pays hautement industrialisés comme l'Australie et le Canada peuvent être presque totalement dépendantes des salaires provenant de l'exploitation forestière commerciale.

À la suite des conclusions de Newton et al. (2016) sur la nécessité d'adapter la définition aux objectifs de l'étude, celle-ci devrait être élargie pour couvrir certaines autres catégories de personnes aux fins d'évaluation de la vulnérabilité.

Compte tenu des services écosystémiques fournis par les forêts et les arbres à la production agricole, il convient d'inclure une quatrième catégorie de personnes qui 
dépendent des forêts: d) les personnes dépendant économiquement de systèmes agricoles qui sont fortement tributaires des services fournis par les forêts et les arbres. Par exemple, les agriculteurs qui pratiquent des cultures dont la reproduction dépend des pollinisateurs sauvages, ou les pêcheurs qui dépendent des poissons et des espèces aquatiques dont le cycle de reproduction et de croissance dépend des mangroves, sont tous dépendants des forêts. Cette catégorie est cohérente avec l'interprétation de la dépendance d'un point de vue économique car elle définit plus précisément le degré de dépendance des communautés vivant à proximité des forêts. Souvent, elles dépendent des forêts non seulement pour compléter leur régime alimentaire et leurs moyens d'existence, mais aussi pour fournir les services écosystémiques indispensables aux activités agricoles qui constituent leur principale source de nourriture et de revenus. En outre, cette approche permet de mieux prendre en compte les communautés et les personnes qui dépendent des forêts pour leur système agricole en aval.

En raison de la croissance des chaînes de valeur et du développement des activités de loisirs, il existe également une cinquième catégorie: e) les personnes qui dépendent des services forestiers (y compris pour le tourisme par exemple) et de la transformation et de la distribution des produits forestiers comme source de revenus. Cette catégorie est différente de la catégorie c) de Fisher et al. car ces populations peuvent plus facilement avoir d'autres options, y compris l'utilisation d'une autre forêt par exemple.

Outre la dépendance économique, il existe des populations, y compris dans les zones urbaines, qui pourraient constituer une sixième catégorie: f) les personnes qui dépendent des forêts pour la fourniture de services tels que le refroidissement, la protection contre les inondations ou l'approvisionnement en eau (en quantité et en qualité), qu'elles vivent dans des zones urbaines ou rurales.

Enfin, une septième catégorie pourrait également être incluse dans les évaluations de la vulnérabilité: g) les personnes qui s'intéressent aux forêts et aux arbres, ou à une forêt spécifique, pour des raisons culturelles, religieuses, environnementales, morales ou récréatives. À proprement parler, ces personnes ne sont pas économiquement dépendantes des forêts, mais leur bien-être physique et psychologique peut en être très dépendant.

Adapté de Meybeck, Rose et Gitz, 2019

Les personnes qui dépendent des forêts peuvent être directement touchées par les changements qui se produisent dans la forêt, tels que les incendies de forêt, les risques accrus d'inondation, les effets sur la disponibilité et la qualité de l'eau. Par exemple, dans de nombreux pays, les incendies de forêt représentent un risque majeur pour les populations vivant à proximité des forêts. Ces risques sont susceptibles d'augmenter en fréquence, en intensité et en étendue. En plus des risques directs, les zones brûlées peuvent connaître deux ou trois ans plus tard, à la suite de violentes précipitations, des coulées de débris d'incendie particulièrement destructrices. Selon la situation de la forêt, certains effets peuvent être ressentis à longue distance, notamment du point de vue de la régulation de l'eau. En particulier, la fréquence et l'intensité croissantes des incendies, des inondations, des glissements de terrain et des pénuries d'eau provoqués par la dégradation ou la gestion non durable des ressources forestières et arboricoles situées dans et autour des villes, affectent gravement les moyens d'existence et la vie des communautés urbaines du monde entier.

Les effets économiques des conséquences du changement climatique sur les forêts et les arbres peuvent être de grande envergure et inclure l'économie non formelle et l'autoconsommation. Les effets économiques des impacts du climat sur une seule espèce, domestiquée ou non, peuvent être évalués assez facilement. Dans certains cas, comme pour la production de bois ou de produits à base d'arbres, des estimations simples des conséquences économiques d'une baisse de rendement peuvent être faites. La production de biomasse d'une forêt spécifique dans des conditions modifiées peut également 
être modélisée avec une production économique de bois dérivée. Ces résultats peuvent être utilisés pour estimer les effets potentiels sur les moyens d'existence des populations, non seulement celles qui bénéficient directement de la collecte de la récolte, mais aussi le long des chaînes de valeur. Dans certains cas, l'impact d'une seule production est tel qu'il peut également avoir des effets indirects au niveau communautaire, en raison de la réduction générale des revenus. Les citadins, par exemple, dépendent des ressources forestières pour l'approvisionnement en eau de qualité, en du bois d'œuvre, en bois de chauffage et en aliments fournis par les forêts entourant leurs villes.

Les effets sur les espèces présentant un intérêt pour le tourisme ou la chasse récréative peuvent être estimés selon le même type d'approche, en partant du nombre de personnes intéressées par l'espèce ou l'activité, par exemple en utilisant le nombre de chasseurs récréatifs enregistrés dans les pays où un tel enregistrement existe ou en utilisant le nombre d'entrées dans les parcs nationaux. Les effets de la modification de la composition d'une forêt peuvent être plus difficiles à quantifier précisément parce qu'ils couvrent généralement un large éventail de biens et de services, dont beaucoup ne sont pas quantifiés économiquement.

Les impacts du changement climatique sur une forêt peuvent nécessiter des interventions correctives telles que la restriction de l'accès pour la protéger ou pour protéger une espèce spécifique, ou pour réduire les risques d'incendie, etc. Ces mesures, à leur tour, affecteront les populations qui dépendent des forêts et justifient donc d'être incluses dans le champ de l'analyse.

Les répercussions sur les populations qui dépendent des forêts varieront selon leur vulnérabilité individuelle, qui dépendrait de leur exposition et de leur sensibilité aux conséquences des effets du changement climatique sur les forêts, ainsi que de leur capacité d'adaptation, qui relève, pour la plupart, d'autres facteurs que la forêt elle-même (notamment les facteurs institutionnels et les droits d'accès). Quatre points présentent un intérêt particulier: l'importance des ressources forestières dans les moyens d'existence généraux, les sources alternatives de revenus et de nourriture et leur niveau de vulnérabilité au changement climatique et à d'autres stress, les indicateurs généraux de vulnérabilité tels que la pauvreté et le niveau d'éducation, et les facteurs institutionnels. L'absence de sécurité des droits d'occupation et d'accès est un facteur supplémentaire de vulnérabilité. Par exemple, cette analyse pourrait inclure des aspects liés à la pauvreté, à l'absence de droits d'accès et de contrôle des ressources forestières et au faible soutien institutionnel des gouvernements aux communautés indigènes et aux populations locales. Un pourcentage important de forêts tropicales se trouve sur des terres communales/ indigènes où la majorité des populations vivent sous le seuil de pauvreté. En effet, les causes sous-jacentes de la vulnérabilité et la manière dont cette question devrait être traitée dans le PNA requièrent l'attention appropriée, car les divers facteurs de vulnérabilité sont souvent concentrés dans des groupes spécifiques. Les filets de sécurité et les systèmes de protection sociale, qu'ils soient institutionnalisés ou qu'ils résultent de formes traditionnelles de solidarité, peuvent réduire la vulnérabilité au niveau des ménages et doivent être évalués au cas par cas. En fait, les systèmes communautaires traditionnels qui sont plus réactifs peuvent être submergés par un choc, comme une sécheresse, qui touche l'ensemble de la communauté.

Une attention particulière doit être accordée aux effets potentiels sur les groupes les plus vulnérables:

- les femmes

- les populations indigènes

- les personnes marginalisées

- les groupes qui dépendent d'une ressource particulièrement menacée.

Les femmes et les hommes ont une relation différente avec les forêts, ainsi que des vulnérabilités et des capacités d'adaptation différentes (Balikoowa et al., 2018). Les rôles culturels, domestiques et économiques divers que les femmes et les hommes jouent dans leurs ménages et leurs communautés influencent les ensembles de connaissances qu'ils développent sur les ressources forestières et arboricoles et sur l'environnement (Bee, 2016). Au niveau mondial, on observe une différenciation significative entre les sexes en ce qui concerne la collecte des produits forestiers, avec d'importantes différences régionales (Sunderland et al., 2014). Ces différences de connaissances et de rôles contribuent à des capacités et des stratégies d'adaptation différenciées face à une base de ressources naturelles en évolution (Djoudi et Brockhaus, 2011). 
Les inégalités entre les sexes et les normes limitant l'accès et le contrôle des femmes sur les ressources, telles que la terre, le capital et les services techniques, peuvent entraver leur capacité à relever les défis du changement climatique (Brody et al., 2008; Lambrou et Piana, 2006; Rodenberg, 2009). Pour ces raisons, les vulnérabilités spécifiques doivent être pleinement intégrées dans le processus du PNA (voir l'exemple de l'Ouganda dans l'encadré 8).

\section{ENCADRÉ 8.}

\section{Un processus de PNA pour l'agriculture sensible au genre en Ouganda}

Le changement climatique a un impact sur tous les membres de la société ougandaise. Cependant, les inégalités systémiques entre les sexes à tous les niveaux, largement discriminatoires à l'égard des femmes et privilégiant les hommes, rendent les femmes plus vulnérables aux effets du changement climatique. Les relations de pouvoir inégales au sein des ménages, notamment dans les domaines de la prise de décision, de l'accès et du contrôle des actifs et des ressources financières, de l'accès aux connaissances pour des pratiques agricoles durables, du travail ménager non rémunéré ainsi que des lois, politiques et structures institutionnelles discriminatoires, limitent les possibilités des femmes.

La prise de conscience de l'importance du genre dans le changement climatique est croissante et le cadre politique pour la promotion de l'égalité des sexes en Ouganda est favorable. Dans ce contexte, le gouvernement ougandais a adopté une approche sensible au genre pour élaborer un PNA pour le secteur de l'agriculture. Le but ultime de cette approche est d'améliorer la capacité des femmes et des hommes à répondre aux effets du changement climatique et de renforcer leur résilience.

Le processus de planification de l'adaptation de l'agriculture en fonction du genre a été mené par le Ministère de l'agriculture, des industries animales et de la pêche (MAAIF), par l'intermédiaire de son groupe de travail sur le changement climatique. Une assistance technique a été fournie par la FAO et le PNUD, en collaboration avec le Département du Changement climatique (CCD) du Ministère de l'eau et de l'environnement (MWE).

Le processus participatif a impliqué des actions tout au long du cycle du PNA, notamment:

- Bilan pour clarifier les écarts entre les sexes dans l'agriculture (Élément A): le groupe de travail sur le changement climatique a identifié et formulé un domaine thématique pour traiter les écarts entre les sexes et faciliter l'élaboration et la mise en œuvre d'un PNA tenant compte de la dimension de genre pour les secteurs agricoles.

- Analyse et consultations des parties prenantes (Élément B): cet élément a permis d'identifier la nécessité de renforcer les capacités en matière d'analyse de genre.

- Ateliers pour renforcer les capacités en matière d'égalité des sexes à tous les niveaux (Élément C): quatre ateliers ont été organisés à l'intention des parlementaires, du gouvernement central, des gouvernements locaux de district et des acteurs non étatiques. Les sujets abordés étaient les suivants: analyse des politiques sensibles à la dimension de genre, et élaboration, planification, budgétisation et mise en œuvre de politiques sensibles à la dimension de genre. 
Le processus de planification sensible au genre a abouti à un PNA pour les secteurs agricoles qui est sensible au genre; le genre est l'un des huit thèmes prioritaires du plan et est inclus dans le budget et le cadre de suivi et d'évaluation. Le processus a également eu des retombées positives, telles qu'une meilleure collaboration entre les ministères et une amélioration des compétences des principaux acteurs au niveau du parlement, du pays, des districts, des acteurs non étatiques, du secteur privé et des communautés locales. L'expérience de l'Ouganda fait ressortir les recommandations suivantes:

- Utiliser une approche participative et inclusive dans les processus de planification de l'adaptation afin de saisir les diverses perspectives des femmes et des hommes de différents milieux socio-économiques et régionaux et de tous les niveaux de gouvernement, y compris les organisations communautaires, locales, centrales et de la société civile.

- Rendre opérationnels les lois, règlements et directives existants qui traitent des questions de genre, tels que les règlements budgétaires.

- S'appuyer sur les recherches et les preuves concernant les vulnérabilités et les impacts liés au genre afin d'améliorer la sensibilisation et l'acceptation de la prise en compte du genre dans différents secteurs.

- Développer les capacités institutionnelles/du personnel en matière d'analyse et d'intégration de la dimension de genre pour le gouvernement et les autres parties prenantes non étatiques. Le développement des capacités en matière de genre favorise la représentation et la participation équitables des femmes dans la mise en œuvre des interventions liées au changement climatique.

- Renforcer les partenariats avec les acteurs étatiques et non étatiques afin de soutenir l'intégration de la dimension de genre et les actions spécifiques au genre et la mobilisation des coûts.

\section{Leçons apprises:}

- En Ouganda, le gouvernement local met en œuvre des mesures de lutte contre le changement climatique tandis que le gouvernement central joue un rôle plus important dans l'élaboration des politiques. Cela nécessite l'engagement des différents niveaux de gouvernement dans le processus de planification.

- Le renforcement de la planification et de la budgétisation sensibles au genre pour l'adaptation dans le secteur agricole nécessite de reconnaître et de s'appuyer sur les mandats et les capacités des différents acteurs et d'adapter le développement des capacités.

- Les institutions culturelles et religieuses sont essentielles pour promouvoir et faire progresser l'égalité des sexes et la gestion des ressources naturelles au niveau communautaire.

- L'approche de l'Ouganda a été particulièrement réussie parce qu'il s'agissait d'un effort soutenu sur une période de plusieurs années avec des experts en matière de genre assurant la cohérence et la coordination. L'approche ougandaise s'est appuyée sur les données existantes pour justifier l'inclusion de la dimension de genre dans le PNA pour l'agriculture et s'est également inspirée des mandats politiques 
existants (en particulier la circulaire d'appel budgétaire sur le genre) pour identifier des moyens de mise en œuvre tenant compte de la dimension genre. En outre, elle a établi un lien entre les actions liées au genre dans les différents éléments du processus du PNA, en s'appuyant sur les contributions des parties prenantes en matière de genre à différents niveaux de planification tout au long du processus.

\section{Informations complémentaires}

Gender and adaptation planning in the agriculture sectors - the case of Uganda: (La dimension de genre dans la planification de l'adaptation des secteurs agricoles - le cas de l'Ouganda): http://www.fao.org/3/I8282EN/i8282en.pdf

Vidéo montrant des planificateurs ougandais abordant la question du genre dans les politiques de changement climatique dans le domaine de l'agriculture: https:// youtu.be/nsIxsSOXups

Equal footing: travailler ensemble pour faire face au changement climatique en Ouganda: https://youtu.be/34G2Zzq3_oo

Compte tenu des multiples rôles des forêts en tant que sources de revenus, d'aliments nutritifs et de bois de cuisson, et en tant que fournisseurs de fonctions de soutien à l'agriculture (Groupe d'experts de haut niveau, 2017), il est recommandé d'examiner plus en profondeur les impacts du changement climatique sur la sécurité alimentaire et la nutrition dans ses quatre dimensions de disponibilité, d'accès, d'utilisation et de stabilité, en particulier dans les zones où l'insécurité alimentaire et les carences nutritionnelles sont répandues.

\subsection{Identifier les domaines dans lesquels les forêts et les arbres peuvent contribuer à renforcer l'adaptation au changement climatique dans les secteurs vulnérables identifiés}

Cette section fournit brièvement des exemples de certaines des contributions que les forêts et les arbres peuvent apporter à l'adaptation:

- les systèmes agricoles (cultures, bétail);

- la régulation du cycle de l'eau;

- le contrôle de l'érosion;

- la protection des zones côtières contre les conséquences de la montée du niveau de la mer (y compris les mangroves);

- les effets de refroidissement pour les villes (y compris les forêts urbaines);

- les effets de refroidissement pour les rivières et la pêche intérieure.

L'objectif est de soutenir la conception de mesures d'adaptation utilisant les forêts, les arbres et l'agroforesterie pour remédier à certaines des vulnérabilités identifiées dans d'autres secteurs. Les tableaux suivants ont été établis sur la base de l'analyse des PNA publiés et de documents comparables complétés par la littérature scientifique et technique pertinente. Comme elles sont organisées par systèmes ou secteurs, certaines mesures peuvent réapparaître dans plusieurs tableaux. L'objectif est d'alimenter les discussions au sein des groupes concernés. 


\section{Cultures et bétail}

\begin{tabular}{|c|c|c|c|}
\hline $\begin{array}{l}\text { CHANGEMENTS } \\
\text { CLIMATIQUES }\end{array}$ & RISQUES CONNEXES & $\begin{array}{l}\text { IMPACTS LIÉS AUX CULTURES } \\
\text { ET AU BÉTAIL }\end{array}$ & $\begin{array}{l}\text { SOLUTIONS AXÉES SUR LES FORÊTS ET LES } \\
\text { ARBRES }\end{array}$ \\
\hline $\begin{array}{l}\text { Climat changeant et } \\
\text { plus variable. }\end{array}$ & & $\begin{array}{l}\text { Réduction des rendements } \\
\text { et de la productivité des } \\
\text { animaux. } \\
\text { Perte de récolte. }\end{array}$ & $\begin{array}{l}\text { Diversification de la production. } \\
\text { Les ressources forestières amortissent } \\
\text { les pertes. }\end{array}$ \\
\hline \multirow{3}{*}{$\begin{array}{l}\text { Températures plus } \\
\text { élevées. }\end{array}$} & $\begin{array}{l}\text { Augmentation de } \\
\text { l'évapotranspiration. }\end{array}$ & Réduction du rendement. & $\begin{array}{l}\text { Systèmes agroforestiers - espèces } \\
\text { à faible demande en eau - dans les } \\
\text { systèmes d'ombrage (pour ombrager } \\
\text { les cultures). }\end{array}$ \\
\hline & $\begin{array}{l}\text { Augmentation de la } \\
\text { fréquence des vagues de } \\
\text { chaleur. }\end{array}$ & $\begin{array}{l}\text { Réduction des rendements } \\
\text { et de la productivité des } \\
\text { animaux. }\end{array}$ & $\begin{array}{l}\text { Systèmes d'agroforesterie sous } \\
\text { l'ombre (pour protéger les cultures et } \\
\text { le bétail). }\end{array}$ \\
\hline & $\begin{array}{l}\text { Augmentation de } \\
\text { l'évaporation. }\end{array}$ & $\begin{array}{l}\text { Baisse des ressources en } \\
\text { eau. }\end{array}$ & $\begin{array}{l}\text { Conserver et établir des arbres le long } \\
\text { des rivières, des canaux d'irrigation et } \\
\text { des réservoirs d'eau pour fournir de } \\
\text { l'ombre. }\end{array}$ \\
\hline \multirow[t]{2}{*}{$\begin{array}{l}\text { Des précipitations } \\
\text { réduites et plus } \\
\text { variables. }\end{array}$} & \multirow[t]{2}{*}{ Sécheresse. } & $\begin{array}{l}\text { Dégradation des sols. } \\
\text { Réduction du rendement } \\
\text { (cultures, prairies). }\end{array}$ & $\begin{array}{l}\text { Systèmes agroforestiers - espèces à } \\
\text { faible demande en eau - y compris sur } \\
\text { les courbes de niveau (pour augmenter } \\
\text { les taux d'infiltration d'eau et la } \\
\text { capacité de rétention d'eau). }\end{array}$ \\
\hline & & $\begin{array}{l}\text { Réduction de la } \\
\text { disponibilité du fourrage. }\end{array}$ & $\begin{array}{l}\text { Systèmes agroforestiers pour la } \\
\text { production de fourrage. }\end{array}$ \\
\hline $\begin{array}{l}\text { Précipitations plus } \\
\text { violentes. }\end{array}$ & $\begin{array}{l}\text { Augmentation de } \\
\text { l'érosion des sols. }\end{array}$ & $\begin{array}{l}\text { Dégradation et érosion des } \\
\text { sols. }\end{array}$ & $\begin{array}{l}\text { Conserver et développer les forêts dans } \\
\text { les zones à haut risque d'érosion. } \\
\text { L'agroforesterie (pour augmenter la } \\
\text { matière organique du sol) et améliorer } \\
\text { le bilan hydrique. }\end{array}$ \\
\hline $\begin{array}{l}\text { Changement des } \\
\text { précipitations de la } \\
\text { neige à la pluie. }\end{array}$ & $\begin{array}{l}\text { Modification du débit } \\
\text { des rivières; plus } \\
\text { grande variabilité des } \\
\text { débits. }\end{array}$ & $\begin{array}{l}\text { Changements dans la } \\
\text { disponibilité des eaux de } \\
\text { surface pour l'irrigation. } \\
\text { Augmentation de la } \\
\text { fréquence des inondations et } \\
\text { des glissements de terrain. }\end{array}$ & $\begin{array}{l}\text { Maintenir et développer la couverture } \\
\text { forestière dans le bassin hydrographique. } \\
\text { Pratiques forestières visant à protéger } \\
\text { et à conserver les bassins versants dans } \\
\text { le but de faciliter l'infiltration dans le } \\
\text { sol et de réduire le ruissellement. }\end{array}$ \\
\hline Vents plus forts. & $\begin{array}{l}\text { Stress des plantes et } \\
\text { des animaux. } \\
\text { Augmentation de } \\
\text { l'érosion des sols. }\end{array}$ & $\begin{array}{l}\text { Réduction des rendements } \\
\text { des cultures et de la } \\
\text { productivité animale. }\end{array}$ & $\begin{array}{l}\text { Les brise-vent et l'agroforesterie (pour } \\
\text { réduire la vitesse des vents). }\end{array}$ \\
\hline $\begin{array}{l}\text { Montée du niveau de } \\
\text { la mer. }\end{array}$ & $\begin{array}{l}\text { Extension des zones à } \\
\text { risque d'inondation. } \\
\text { Salinisation. }\end{array}$ & $\begin{array}{l}\text { Réduction des surfaces } \\
\text { cultivables. }\end{array}$ & $\begin{array}{l}\text { Protection et restauration des } \\
\text { mangroves. } \\
\text { Mettre en place des forêts de } \\
\text { protection du littoral. }\end{array}$ \\
\hline $\begin{array}{l}\text { Changement } \\
\text { climatique. }\end{array}$ & $\begin{array}{l}\text { Réduction des } \\
\text { pollinisateurs sauvages } \\
\text { et domestiqués. } \\
\text { Augmentation des } \\
\text { populations et de } \\
\text { l'activité des insectes } \\
\text { nuisibles. }\end{array}$ & $\begin{array}{l}\text { Réduction des rendements } \\
\text { des cultures pollinisées. }\end{array}$ & $\begin{array}{l}\text { Protection et création de forêts, de } \\
\text { parcelles forestières, de haies et de } \\
\text { systèmes agroforestiers pour fournir } \\
\text { un abri et de la nourriture aux } \\
\text { pollinisateurs sauvages. } \\
\text { Protection et création de forêts, de } \\
\text { parcelles forestières, de haies et de } \\
\text { systèmes agroforestiers agissant } \\
\text { comme des cultures-pièges, } \\
\text { concurrence interspécifique accrue } \\
\text { avec les espèces non nuisibles, accueil } \\
\text { des ennemis naturels des nuisibles }\end{array}$ \\
\hline
\end{tabular}




\section{Pêche et aquaculture}

\begin{tabular}{|c|c|c|c|}
\hline $\begin{array}{l}\text { CHANGEMENTS } \\
\text { CLIMATIQUES }\end{array}$ & RISQUES CONNEXES & $\begin{array}{l}\text { IMPACTS LIÉS AUX CULTURES } \\
\text { ET AU BÉTAIL }\end{array}$ & $\begin{array}{l}\text { SOLUTIONS AXÉES SUR LES FORÊTS ET LES } \\
\text { ARBRES }\end{array}$ \\
\hline \multirow[b]{2}{*}{$\begin{array}{l}\text { Températures plus } \\
\text { élevées. }\end{array}$} & Évaporation. & Moins d'eau disponible. & \multirow[b]{2}{*}{$\begin{array}{l}\text { Conserver et planter des arbres pour } \\
\text { fournir de l'ombre le long des rivières, } \\
\text { pour l'irrigation et le stockage de l'eau. }\end{array}$} \\
\hline & $\begin{array}{l}\text { Température plus élevée } \\
\text { des eaux de surface } \\
\text { (rivières et lacs). }\end{array}$ & Mortalité des poissons. & \\
\hline \multirow[t]{2}{*}{$\begin{array}{l}\text { Précipitations plus } \\
\text { violentes. }\end{array}$} & Erosion accrue. & $\begin{array}{l}\text { Dégradation des zones de } \\
\text { frai des poissons. } \\
\text { Dégradation de la qualité } \\
\text { de l'eau. }\end{array}$ & $\begin{array}{l}\text { Conserver et aménager des forêts } \\
\text { dans les zones les plus menacées par } \\
\text { l'érosion. }\end{array}$ \\
\hline & Erosion accrue. & $\begin{array}{l}\text { Dégradation de la qualité } \\
\text { de l'eau. }\end{array}$ & \multirow{2}{*}{$\begin{array}{l}\text { Maintenir et développer la } \\
\text { couverture forestière dans le bassin } \\
\text { hydrographique pour faciliter } \\
\text { l'infiltration dans le sol et réduire le } \\
\text { ruissellement. }\end{array}$} \\
\hline $\begin{array}{l}\text { Changement des } \\
\text { précipitations de la } \\
\text { neige à la pluie. }\end{array}$ & $\begin{array}{l}\text { Changements dans les } \\
\text { débits des rivières; plus } \\
\text { grande variabilité des } \\
\text { débits. }\end{array}$ & $\begin{array}{l}\text { Inundaciones. } \\
\text { «Lavado» de las zonas de } \\
\text { desove. }\end{array}$ & \\
\hline $\begin{array}{l}\text { Montée du niveau de } \\
\text { la mer. }\end{array}$ & $\begin{array}{l}\text { Les mangroves } \\
\text { menacées. }\end{array}$ & $\begin{array}{l}\text { Dégradation de l'habitat, } \\
\text { critique pour la } \\
\text { reproduction des poissons. }\end{array}$ & Protéger et restaurer les mangroves. \\
\hline
\end{tabular}

\section{Eau}

\begin{tabular}{|c|c|c|c|}
\hline $\begin{array}{l}\text { CHANGEMENTS } \\
\text { CLIMATIQUES }\end{array}$ & RISQUES CONNEXES & $\begin{array}{l}\text { IMPACTS LIÉS AUX GULTURES ET } \\
\text { AU BÉTAIL }\end{array}$ & $\begin{array}{l}\text { SOLUTIONS AXÉES SUR LES FORÊTS ET } \\
\text { LES ARBRES }\end{array}$ \\
\hline \multirow[b]{2}{*}{$\begin{array}{l}\text { Températures plus } \\
\text { élevées. }\end{array}$} & Évaporation. & Réduction des ressources en eau. & \multirow{2}{*}{$\begin{array}{l}\text { Conserver et planter des arbres } \\
\text { le long des rivières, des canaux } \\
\text { d'irrigation et des réservoirs d'eau } \\
\text { pour fournir de l'ombre. }\end{array}$} \\
\hline & $\begin{array}{l}\text { Température plus élevée } \\
\text { des eaux de surface } \\
\text { (rivières et lacs). }\end{array}$ & Mortalité des poissons. & \\
\hline $\begin{array}{l}\text { Des précipitations } \\
\text { réduites et plus } \\
\text { variables. }\end{array}$ & Sécheresse. & $\begin{array}{l}\text { Dégradation des sols. } \\
\text { Réduction du rendement } \\
\text { (cultures, prairies). }\end{array}$ & $\begin{array}{l}\text { Les arbres (espèces et densité } \\
\text { appropriées) peuvent améliorer } \\
\text { la conservation de l'eau et la } \\
\text { circulation dans le sol. }\end{array}$ \\
\hline \multirow[t]{2}{*}{$\begin{array}{l}\text { Précipitations plus } \\
\text { violentes. }\end{array}$} & Érosion accrue. & $\begin{array}{l}\text { Ensablement des barrages. } \\
\text { Dégradation des zones de frai } \\
\text { des poissons. } \\
\text { Réduction de la navigabilité } \\
\text { des rivières. } \\
\text { Dégradation de la qualité de } \\
\text { l'eau. }\end{array}$ & $\begin{array}{l}\text { Conserver et étendre les forêts dans } \\
\text { les zones les plus menacées par } \\
\text { l'érosion. }\end{array}$ \\
\hline & Inondations & $\begin{array}{l}\text { Pertes de vies humaines. } \\
\text { Perte de biens. } \\
\text { Dégradation de la qualité de } \\
\text { l'eau. }\end{array}$ & \multirow{2}{*}{$\begin{array}{l}\text { Maintenir et développer la } \\
\text { couverture forestière dans le bassin } \\
\text { hydrographique pour faciliter } \\
\text { l'infiltration dans le sol et réduire le } \\
\text { ruissellement. }\end{array}$} \\
\hline $\begin{array}{l}\text { Changement des } \\
\text { précipitations de la } \\
\text { neige à la pluie. }\end{array}$ & $\begin{array}{l}\text { Modification du débit } \\
\text { des rivières; plus grande } \\
\text { variabilité des débits. }\end{array}$ & $\begin{array}{l}\text { Inondations. } \\
\text { «Vidage» des zones de frai } \\
\text { des poissons. }\end{array}$ & \\
\hline $\begin{array}{l}\text { Montée du niveau de } \\
\text { la mer. }\end{array}$ & $\begin{array}{l}\text { Modification du profil des } \\
\text { rivières, en particulier } \\
\text { près de la mer. }\end{array}$ & $\begin{array}{l}\text { Expansion des zones à risque } \\
\text { d'inondation. }\end{array}$ & $\begin{array}{l}\text { Mettre en place une stratégie de } \\
\text { protection des forêts. }\end{array}$ \\
\hline
\end{tabular}




\section{Villes, installations humaines, infrastructures}

\begin{tabular}{|c|c|c|c|}
\hline $\begin{array}{l}\text { CHANGEMENTS } \\
\text { CLIMATIQUES }\end{array}$ & RISQUES CONNEXES & $\begin{array}{l}\text { IMPACTS LIÉS AUX CULTURES ET } \\
\text { AU BÉTAIL }\end{array}$ & $\begin{array}{l}\text { SOLUTIONS AXÉES SUR LES FORÊTS ET LES } \\
\text { ARBRES }\end{array}$ \\
\hline $\begin{array}{l}\text { Températures plus } \\
\text { élevées. }\end{array}$ & Vagues de chaleur. & $\begin{array}{l}\text { Risques sanitaires dans les } \\
\text { zones urbaines. }\end{array}$ & $\begin{array}{l}\text { Planter des arbres dans et autour } \\
\text { des zones urbaines pour un effet de } \\
\text { refroidissement et pour réduire les } \\
\text { effets d'îlots de chaleur. }\end{array}$ \\
\hline \multirow[t]{2}{*}{$\begin{array}{l}\text { Précipitations plus } \\
\text { violentes. }\end{array}$} & Inondations. & $\begin{array}{l}\text { Perte de vies et de biens. } \\
\text { Diminution de la qualité de } \\
\text { l'eau. } \\
\text { Maladies liées à l'eau, } \\
\text { diarrhées. }\end{array}$ & $\begin{array}{l}\text { Maintenir et développer la } \\
\text { couverture forestière dans le bassin } \\
\text { hydrographique pour faciliter } \\
\text { l'infiltration dans le sol, réduire le } \\
\text { ruissellement et améliorer la qualité } \\
\text { de l'eau par la filtration des polluants } \\
\text { et des sédiments. }\end{array}$ \\
\hline & Glissements de terrain. & Perte de vies et de biens. & $\begin{array}{l}\text { Planter et entretenir des arbres, en } \\
\text { particulier sur les pentes. }\end{array}$ \\
\hline $\begin{array}{l}\text { Augmentation de } \\
\text { l'érosion éolienne. }\end{array}$ & Pollution de l'air. & $\begin{array}{l}\text { Augmentation des } \\
\text { symptômes respiratoires, } \\
\text { tels que l'irritation des } \\
\text { voies respiratoires, la toux } \\
\text { ou la difficulté à respirer. } \\
\text { Diminution de la fonction } \\
\text { pulmonaire. Mort prématurée } \\
\text { chez les personnes souffrant } \\
\text { de maladies cardiaques ou } \\
\text { pulmonaires. }\end{array}$ & $\begin{array}{l}\text { Conserver et développer les forêts et } \\
\text { les arbres dans les zones exposées } \\
\text { au risque d'érosion éolienne afin de } \\
\text { maintenir la couverture naturelle du } \\
\text { sol et de le rendre moins sensible à } \\
\text { l'érosion éolienne. }\end{array}$ \\
\hline \multirow[t]{2}{*}{$\begin{array}{l}\text { Augmentation de la } \\
\text { fréquence des feux de } \\
\text { forêt. }\end{array}$} & Pollution de l'air. & $\begin{array}{l}\text { Augmentation des } \\
\text { symptômes respiratoires, } \\
\text { tels que l'irritation des } \\
\text { voies respiratoires, la toux } \\
\text { ou la difficulté à respirer. } \\
\text { Diminution de la fonction } \\
\text { pulmonaire. Mort prématurée } \\
\text { chez les personnes souffrant } \\
\text { de maladies cardiaques ou } \\
\text { pulmonaires. }\end{array}$ & \multirow[t]{2}{*}{$\begin{array}{l}\text { Prévenir et gérer les incendies de } \\
\text { forêt. } \\
\text { Gérer correctement l'interface forêt- } \\
\text { zone urbaine afin de minimiser le } \\
\text { risque d'incendie. }\end{array}$} \\
\hline & Dommages directs. & Perte de vies et de biens. & \\
\hline $\begin{array}{l}\text { Montée du niveau de } \\
\text { la mer. }\end{array}$ & Inondations. & Perte de vies et de biens. & $\begin{array}{l}\text { Conserver et restaurer les } \\
\text { mangroves. Créer des forêts côtières. }\end{array}$ \\
\hline
\end{tabular}




\section{Énergie}

\begin{tabular}{|c|c|c|c|}
\hline $\begin{array}{l}\text { CHANGEMENTS } \\
\text { CLIMATIQUES }\end{array}$ & RISQUES CONNEXES & $\begin{array}{l}\text { IMPACTS LIÉS AUX CULTURES ET } \\
\text { AU BÉTAIL }\end{array}$ & $\begin{array}{l}\text { SOLUTIONS AXÉES SUR LES FORÊTS ET } \\
\text { LES ARBRES }\end{array}$ \\
\hline \multirow{2}{*}{$\begin{array}{l}\text { Températures plus } \\
\text { élevées. }\end{array}$} & $\begin{array}{l}\text { Température moyenne } \\
\text { plus élevée en été. }\end{array}$ & $\begin{array}{l}\text { Augmentation de la } \\
\text { consommation d'énergie pour } \\
\text { la climatisation. }\end{array}$ & \multirow{2}{*}{$\begin{array}{l}\text { Planter des arbres et gérer les arbres } \\
\text { dans et autour des zones urbaines } \\
\text { pour un effet de refroidissement } \\
\text { et pour réduire les effets d'îlot } \\
\text { de chaleur; réduire également } \\
\text { l'utilisation de la climatisation. }\end{array}$} \\
\hline & Vagues de chaleur. & $\begin{array}{l}\text { Forte consommation d'énergie } \\
\text { pour la climatisation. }\end{array}$ & \\
\hline $\begin{array}{l}\text { Précipitations plus } \\
\text { violentes. }\end{array}$ & Érosion accrue. & $\begin{array}{l}\text { Ensablement des barrages, } \\
\text { réduction de leur capacité } \\
\text { de stockage d'eau avec des } \\
\text { impacts sur le potentiel } \\
\text { hydroélectrique. }\end{array}$ & $\begin{array}{l}\text { Conserver et développer les forêts } \\
\text { dans les zones les plus exposées au } \\
\text { risque d'érosion. }\end{array}$ \\
\hline $\begin{array}{l}\text { Des précipitations } \\
\text { réduites et plus } \\
\text { variables. }\end{array}$ & \multirow{2}{*}{$\begin{array}{l}\text { Modification du débit des } \\
\text { rivières; } \\
\text { une plus grande } \\
\text { variabilité des débits. }\end{array}$} & \multirow{2}{*}{$\begin{array}{l}\text { Irrégularité du potentiel } \\
\text { hydroélectrique. } \\
\text { Réduction de la disponibilité de } \\
\text { l'eau pour le refroidissement } \\
\text { des installations d'énergie } \\
\text { thermique et nucléaire. }\end{array}$} & \multirow{2}{*}{$\begin{array}{l}\text { Maintenir et développer la } \\
\text { couverture forestière dans le bassin } \\
\text { hydrographique pour faciliter } \\
\text { l'infiltration dans le sol et réduire le } \\
\text { ruissellement. }\end{array}$} \\
\hline $\begin{array}{l}\text { Changement des } \\
\text { précipitations de la } \\
\text { neige à la pluie. }\end{array}$ & & & \\
\hline
\end{tabular}

\section{Santé}

\begin{tabular}{|c|c|c|c|}
\hline CAMBIOS CLIMÁTICOS & RISQUES CONNEXES & $\begin{array}{l}\text { IMPACTS LIÉS AUX CULTURES ET AU } \\
\text { BÉTAIL }\end{array}$ & $\begin{array}{l}\text { SOLUTIONS AXÉES SUR LES FORÊTS ET } \\
\text { LES ARBRES }\end{array}$ \\
\hline \multirow{2}{*}{$\begin{array}{l}\text { Températures plus } \\
\text { élevées. }\end{array}$} & \multirow[b]{2}{*}{ Vagues de chaleur. } & $\begin{array}{l}\text { Risques de santé au travail pour } \\
\text { les agriculteurs et les travailleurs } \\
\text { agricoles. }\end{array}$ & $\begin{array}{l}\text { Planter des arbres dans les champs } \\
\text { pour fournir de l'ombre aux heures } \\
\text { les plus chaudes de la journée. }\end{array}$ \\
\hline & & $\begin{array}{l}\text { Risques sanitaires dans les zones } \\
\text { urbaines. }\end{array}$ & $\begin{array}{l}\text { Planter des arbres dans et autour } \\
\text { des zones urbaines pour un effet de } \\
\text { refroidissement et pour réduire les } \\
\text { effets d'îlots de chaleur. }\end{array}$ \\
\hline $\begin{array}{l}\text { Précipitations plus } \\
\text { violentes. }\end{array}$ & Inondations. & Maladies liées à l'eau, diarrhées. & $\begin{array}{l}\text { Maintenir et développer la } \\
\text { couverture forestière dans le bassin } \\
\text { hydrographique pour faciliter } \\
\text { l'infiltration dans le sol et réduire le } \\
\text { ruissellement. }\end{array}$ \\
\hline $\begin{array}{l}\text { Augmentation de } \\
\text { l'érosion éolienne. }\end{array}$ & Pollution de l'air. & $\begin{array}{l}\text { Augmentation des symptômes } \\
\text { respiratoires, tels que l'irritation } \\
\text { des voies respiratoires, la toux ou } \\
\text { la difficulté à respirer. Diminution } \\
\text { de la fonction pulmonaire. Mort } \\
\text { prématurée chez les personnes } \\
\text { souffrant de maladies cardiaques } \\
\text { ou pulmonaires. }\end{array}$ & $\begin{array}{l}\text { Conserver et développer les forêts } \\
\text { dans les zones les plus exposées au } \\
\text { risque d'érosion éolienne. }\end{array}$ \\
\hline $\begin{array}{l}\text { Augmentation de la } \\
\text { fréquence des feux } \\
\text { de forêt. }\end{array}$ & Pollution de l'air. & $\begin{array}{l}\text { Augmentation des symptômes } \\
\text { respiratoires, tels que l'irritation } \\
\text { des voies respiratoires, la toux ou } \\
\text { la difficulté à respirer. Diminution } \\
\text { de la fonction pulmonaire. Mort } \\
\text { prématurée chez les personnes } \\
\text { souffrant de maladies cardiaques } \\
\text { ou pulmonaires }\end{array}$ & $\begin{array}{l}\text { Prévenir et gérer les incendies de } \\
\text { forêt. } \\
\text { Gérer correctement l'interface forêt- } \\
\text { zone urbaine afin de minimiser le } \\
\text { risque d'incendie de forêt. }\end{array}$ \\
\hline
\end{tabular}


Dans son PNA pour les secteurs agricoles, l'Ouganda accorde une importance particulière au rôle de la foresterie dans l'adaptation de tous les secteurs agricoles (voir encadré 9).

\section{ENCADRÉ 9 .}

\section{La foresterie dans le PNA de l'agriculture de l'Ouganda}

La foresterie est l'un des domaines prioritaires pour les mesures d'adaptation dans le Plan national d'Adaptation du Secteur agricole de l'Ouganda. Bien que la contribution des forêts au PIB agricole ne soit pas importante - seulement 4 pour cent - le plan reconnaitt l'importance de l'agriculture pour maintenir des environnements résistants au climat qui soutiennent la productivité agricole et l'adaptation. Au cours de l'analyse, le plan adopte une approche «forêts contre adaptation»:

- La perte de forêts et la dégradation des terres. Les plus grandes menaces environnementales en Ouganda sont la destruction durable des zones humides, la déforestation et la contamination des lacs. Chaque année, l'Ouganda perd plus de 2 pour cent de sa couverture forestière et de ses zones humides. Les estimations de la perte économique attribuée à la dégradation des terres en Ouganda dans les années 1990 varient entre 4 et 12 pour cent du PIB.

- Conversion à l'agriculture. La productivité des cultures est en baisse et la superficie des terres agricoles a augmenté de 1 pour cent par an au cours de la dernière décennie, ce qui signifie que d'ici 2040, plus de 90 pour cent des terres de l'Ouganda seront utilisées pour l'agriculture, menaçant ainsi les forêts et les zones humides. Afin d'éviter les risques liés à ces changements, l'agriculture ougandaise doit passer d'une utilisation extensive des terres à une agriculture intensive.

- Les forêts comme combustible. Les Ougandais dépendent largement de l'énergie traditionnelle de la biomasse, qui est déjà en pénurie en raison des taux élevés de déforestation. La déforestation causée par une demande accrue de biomasse augmente la dégradation des terres, ce qui réduit également la productivité agricole. Le changement climatique va probablement réduire la disponibilité de la biomasse, c'est pourquoi il convient de donner la priorité à la transition vers des sources d'énergie alternatives pour l'usage domestique.

- Garantir la disponibilité de l'eau pour l'agriculture. Les forêts font partie des actions prioritaires relatives à la disponibilité future de l'eau pour l'agriculture et d'autres utilisations. Cela est considéré comme faisant partie intégrante de l'amélioration des systèmes d'utilisation des terres et de la réduction de la dégradation des forêts, des sols et de l'environnement. Les mesures prises à cette fin comprennent le renforcement de la gestion des bassins versants dans la planification agricole, l'adoption d'une gestion durable des terres agricoles, des terres forestières et de l'eau afin de réduire la dégradation, et la promotion de pratiques de gestion des forêts et des écosystèmes appropriées afin d'accroître la résilience des communautés paysannes face aux effets du changement climatique. 


\subsection{Identifier les options d'adaptation pour les forêts, les arbres et les personnes qui dépendent des forêts}

Après l'identification des risques et des vulnérabilités pour les forêts et les arbres ainsi que pour les personnes qui dépendent de la forêt, les voies et les mesures d'adaptation peuvent être esquissées. Ces voies et mesures devront également tenir compte d'autres pressions exercées sur les forêts ainsi que d'autres objectifs, notamment la contribution potentielle à l'adaptation d'autres secteurs (voir section 6.3).

Le maintien des écosystèmes forestiers dans un état sain est la mesure la plus simple à prendre pour conserver leur résilience (FAO, 2016a). Des forêts en bonne santé sont mieux à même de faire face au stress, de se remettre des dommages et de s'adapter de manière autonome aux changements. Les écosystèmes sains sont plus résistants aux influences biotiques et abiotiques négatives que les écosystèmes soumis à un stress et dont les processus écologiques sont altérés. Les bonnes pratiques incluent la lutte intégrée contre les insectes ravageurs, la gestion des incendies de forêt, l'utilisation de l'exploitation à faible impact (RIL) dans les forêts de production, la limitation de la collecte de produits forestiers autres que le bois ou du pâturage du bétail dans les forêts à des niveaux durables, et l'application de la législation forestière. La restauration des forêts dégradées à un état sain, rétablissant ainsi les fonctions des écosystèmes, est une stratégie majeure pour accroître la résilience. On estime que deux milliards d'hectares de terres peuvent potentiellement être restaurés ou reboisés. La biodiversité est un facteur clé de la résilience des écosystèmes forestiers et des arbres face aux stress existants et constitue un ingrédient de base pour renforcer leur capacité d'adaptation face aux futurs facteurs de stress. Une attention particulière est accordée à l'AbE, qui est la gestion des écosystèmes et des services écosystémiques pour réduire la vulnérabilité humaine au changement climatique. Cela nécessite une meilleure surveillance de l'état des forêts et des effets du changement climatique sur celles-ci, y compris la surveillance des ravageurs indigènes et des espèces envahissantes.

La gestion adaptative des forêts est essentielle pour réduire la vulnérabilité des forêts et maintenir leur productivité. Les mesures d'adaptation peuvent comprendre, par exemple, la sélection d'espèces tolérantes à la chaleur et à la sécheresse dans les forêts plantées, la plantation de matériel végétal de diverses provenances, la plantation de variétés d'arbres adaptées aux conditions climatiques prévues et la régénération naturelle assistée d'espèces et de variétés adaptées. Les effets probables du changement climatique sur la croissance des arbres et des forêts lors de la détermination des sites et de la sélection des espèces pour l'établissement de tous les types de forêts et de systèmes arborés doivent être examinés de près. Le tableau 7 ci-dessous indique quelques-unes des principales mesures d'adaptation qui peuvent être incluses. Des options plus détaillées sont disponibles dans les directives de la FAO sur le changement climatique à l'intention des gestionnaires de forêts (FAO, 2013). 


\section{TABLEAU 2.}

\section{Exemples de mesures visant à accroître la résilience des forêts face aux divers impacts du changement climatique}

\begin{tabular}{|c|c|c|}
\hline RISQUES/IMPACTS & $\begin{array}{l}\text { IMPLICATIONS (SOCIALES, ÉCONOMIQUES, } \\
\text { ENVIRONNEMENTALES) }\end{array}$ & $\begin{array}{l}\text { MESURES D'INTERVENTION POUR LA RÉDUCTION } \\
\text { DES RISQUES ET L'AUGMENTATION DE LA } \\
\text { RÉSILIENCE }\end{array}$ \\
\hline $\begin{array}{l}\text { Diminution de la vitalité } \\
\text { et de la productivité des } \\
\text { forêts }\end{array}$ & $\begin{array}{l}\text { Diminution des revenus tirés des produits } \\
\text { forestiers ligneux et non ligneux; réduction } \\
\text { des services écosystémiques forestiers. }\end{array}$ & $\begin{array}{l}\text { Ajuster les pratiques sylvicoles. Modifier } \\
\text { la composition des espèces et des variétés; } \\
\text { augmenter la biodiversité des forêts; mettre } \\
\text { en œuvre des mesures de restauration des } \\
\text { forêts. }\end{array}$ \\
\hline $\begin{array}{l}\text { Augmentation des } \\
\text { parasites et des maladies } \\
\text { des forêts }\end{array}$ & $\begin{array}{l}\text { Réduction des revenus forestiers; réduction } \\
\text { des services écosystémiques forestiers. }\end{array}$ & $\begin{array}{l}\text { Mettre en œuvre et intensifier les mesures } \\
\text { de mesures de gestion des ravageurs et des } \\
\text { maladies; adapter les pratiques sylvicoles. }\end{array}$ \\
\hline $\begin{array}{l}\text { Augmentation des } \\
\text { incendies de forêt }\end{array}$ & $\begin{array}{l}\text { Pertes de vies humaines; dommages aux } \\
\text { infrastructures; réduction des revenus } \\
\text { forestiers et des services écosystémiques; } \\
\text { pertes de faune et de flore. }\end{array}$ & $\begin{array}{l}\text { Mettre en œuvre et intensifier la gestion des } \\
\text { incendies de forêt en adaptant les pratiques } \\
\text { sylvicoles. }\end{array}$ \\
\hline $\begin{array}{l}\text { Augmentation de l'érosion } \\
\text { hydrique et glissements } \\
\text { de terrain }\end{array}$ & $\begin{array}{l}\text { Dommages aux forêts et aux infrastructures } \\
\text { (villes, routes, barrages); réduction de la } \\
\text { qualité de l'eau. }\end{array}$ & $\begin{array}{l}\text { Entreprendre des mesures de gestion des } \\
\text { bassins versants (y compris la protection et } \\
\text { l'augmentation de la couverture végétale: } \\
\text { réduction de l'intensité de la récolte et des } \\
\text { autres utilisations). }\end{array}$ \\
\hline $\begin{array}{l}\text { Dépérissement des forêts/ } \\
\text { arbres et dégradation } \\
\text { des sols induits par la } \\
\text { sécheresse }\end{array}$ & $\begin{array}{l}\text { Réduction de la disponibilité des produits } \\
\text { forestiers. } \\
\text { Augmentation des dommages causés par le } \\
\text { vent; réduction des valeurs de pâturage. }\end{array}$ & $\begin{array}{l}\text { Planter des brise-vent; maintenir le couvert } \\
\text { des arbres; modifier la composition des } \\
\text { espèces et des variétés. }\end{array}$ \\
\hline $\begin{array}{l}\text { Augmentation des } \\
\text { dommages causés par les } \\
\text { tempêtes }\end{array}$ & $\begin{array}{l}\text { Réduction des revenus forestiers et des } \\
\text { services écosystémiques; risque accru de } \\
\text { parasites et de maladies. }\end{array}$ & $\begin{array}{l}\text { Changement d'espèces et adaptation de la } \\
\text { taille des arbres pour réduire les risques; } \\
\text { récolte de sauvetage; lutte contre les parasites } \\
\text { et les maladies. }\end{array}$ \\
\hline $\begin{array}{l}\text { Réduction de l'étendue } \\
\text { et de la vitalité des } \\
\text { mangroves et des forêts } \\
\text { côtières }\end{array}$ & $\begin{array}{l}\text { Exposition accrue des terres aux dommages } \\
\text { causés par les tempêtes; réduction de la } \\
\text { productivité des pêcheries côtières. }\end{array}$ & $\begin{array}{l}\text { Accroître la protection, la restauration et } \\
\text { et la mise en valeur des mangroves et autres } \\
\text { forêts côtières. }\end{array}$ \\
\hline $\begin{array}{l}\text { Modification de l'aire de } \\
\text { répartition des espèces et } \\
\text { extinctions d'espèces }\end{array}$ & $\begin{array}{l}\text { Réduction des fonctions des écosystèmes } \\
\text { forestiers; perte de la biodiversité forestière. }\end{array}$ & $\begin{array}{l}\text { Restaurer/accroître la connectivité des forêts } \\
\text { et des corridors de la faune sauvage; favoriser } \\
\text { la migration; prendre des mesures de } \\
\text { conservation ex-situ. }\end{array}$ \\
\hline
\end{tabular}


Les gestionnaires de forêts doivent tenir compte de nombreux facteurs et objectifs (voir figure 5). Le changement climatique en est un de plus. Il modifie également la plupart des autres facteurs. Les options d'adaptation doivent donc être comprises comme faisant partie de la gestion durable des forêts et, par conséquent, être intégrées dans le programme forestier national du pays. Leur contribution potentielle au développement durable et les avantages connexes de l'atténuation, ainsi que les éventuelles conséquences sociales, devraient également être pris en considération. Une compréhension approfondie des coûts et des avantages des options, en particulier de leur répartition dans le temps et entre les catégories d'acteurs, est particulièrement importante pour leur faisabilité. Il a également été noté que le montant du financement disponible pour l'adaptation peut être un facteur critique dans le choix des options visant à minimiser l'extinction des espèces dans un biome d'Afrique du Sud (Wintle et al., 2011): avec un petit budget, la gestion des incendies était la meilleure option; avec un budget intermédiaire, la meilleure stratégie était de protéger davantage les habitats; avec un budget plus important, la meilleure option est de mettre plus d'argent dans la protection contre les incendies.

\section{FIGURE 5.}

\section{Les gestionnaires des forêts réagissent à un large éventail de facteurs}

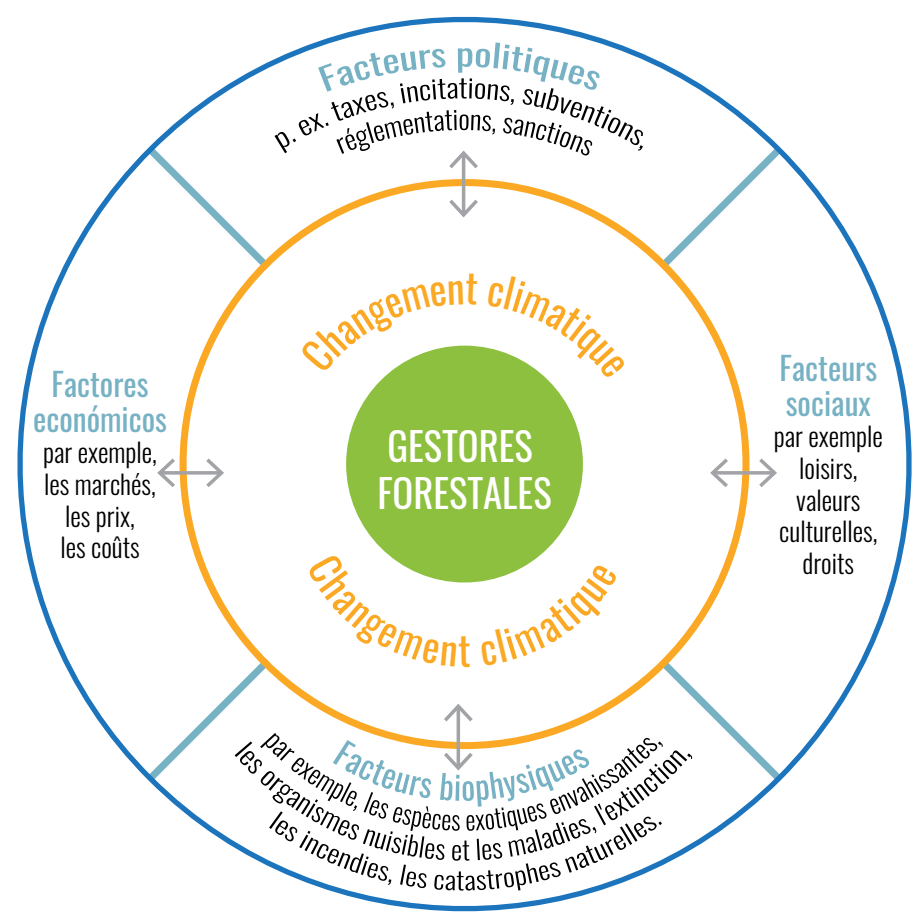

Source: FAO, 2013

L'identification des options d'adaptation doit être accompagnée d'une estimation de leurs coûts et avantages (voir encadré 10). 
ENGADRÉ 10.

Évaluation de la viabilité financière et économique des options d'adaptation liées à l'agroforesterie pour les agriculteurs kenyans

L'agroforesterie est considérée comme l'une des meilleures options pour les agriculteurs du Kenya pour s'adapter aux risques du changement climatique. Selon les changements prévus des températures moyennes saisonnières et les impacts attendus sur le cycle des courtes pluies, les conditions de sécheresse entraînant des pertes de récoltes et de bétail devraient s'intensifier. L'agroforesterie - qui consiste à faire pousser des cultures en même temps que des arbres pour faire de l'ombre et du bois - aurait un impact positif sur les moyens d'existence (Nyaruai, 2016). L'agroforesterie offre la possibilité de diversifier les sources de revenus des ménages grâce à la production de fruits, de fourrage, de bois de chauffage et de construction, de substances médicales, de fibres et de cire.

Le PNA du Kenya propose une série d'actions pour 2015-2030 dans le domaine de l'agriculture. Le succès de leur mise en œuvre dépend des ajustements que les agriculteurs devront faire pour adopter de nouvelles technologies, réaffecter la maind'œuvre et réaliser des investissements. Il est donc important de s'attaquer aux obstacles à l'adoption. En 2018, dans le cadre du programme PNA -Ag, une étude a été réalisée à l'aide d'une analyse coûts-avantages afin de comparer les coûts et les avantages financiers et économiques de l'adoption de pratiques d'adaptation (notamment l'agroforesterie et la conservation des sols et de l'eau). Ces ensembles de pratiques sont déjà courants - une enquête menée auprès de 642 ménages dans 5 comtés a montré que 89 pour cent des agriculteurs plantent des arbres dans leurs champs.

Toutefois, il existe divers obstacles à une adoption plus généralisée. L'étude, qui s'appuie sur une enquête représentative et des données secondaires, a montré que les agriculteurs en tirent des avantages économiques et financiers évidents à long terme. Néanmoins, lorsque les arbres arrivent à maturité, il y a un compromis avec la productivité des cultures adjacentes. Par exemple, il a été constaté qu'au Kenya oriental, le maïs planté sur les mêmes parcelles que les plantes ligneuses avaient un rendement inférieur de 30 pour cent à celui des champs sans arbres (Ndlovu, 2013). Néanmoins, les espèces ligneuses procuraient des revenus substantiels aux agriculteurs (entre 50 et 80 USD selon la taille et la qualité) lorsqu'elles étaient récoltées après 8 ans. Par conséquent, ces revenus peuvent compenser les pertes de revenus annuelles dues à une baisse de rendement en générant un bénéfice supplémentaire positif pour les agriculteurs, ainsi qu'une série d'avantages environnementaux pour la société dans son ensemble.

Cependant, la rentabilité des techniques agricoles ne suffit pas à garantir l'adoption des pratiques d'adaptation souhaitables. Les exemples fournis dans le cas du Kenya ont montré certains des moyens par lesquels les gouvernements peuvent faciliter cela:

- sensibilisant á l'agriculture et en améliorant l'accès;

- en établissant la sécurité du régime foncier et en s'attaquant aux facteurs d'adoption;

- en accroissant les intrants productifs et le financement;

- en améliorant l'accès au crédit.

Pour plus d'informations, FAO \& PNUD, 2020b. 


\subsection{Identifier les moyens de rendre l'environnement plus favorable}

Les impacts du changement climatique sur les forêts doivent être abordés et gérés à différentes échelles, ce qui nécessite une action au niveau du paysage, au niveau infranational et/ou national, et par différents acteurs de manière coordonnée. Pour ce faire, il convient de mettre en place un environnement propice approprié et de mobiliser de manière cohérente les leviers techniques, juridictionnels et financiers. Un bon exemple est la prévention des incendies, la détection précoce et l'action rapide, qui nécessitent toutes une action collective organisée aux niveaux du paysage infranational et national. La publication préparée par la FAO qui décrit avec précision tous les principes et mesures à mettre en œuvre, La gestion des incendies: Recommandations volontaires pour la gestion des incendies - Principes directeurs et actions stratégiques est disponible dans les six langues des Nations Unies (FAO, 2006).

La prévention et la surveillance des organismes nuisibles sont une autre question importante à résoudre ${ }^{6}$. Plusieurs pays ${ }^{7}$ ont mis en place un système de surveillance des organismes nuisibles proposant des outils pour identifier les organismes nuisibles, une base de données en ligne, des moyens pour signaler les foyers d'organismes nuisibles et des directives pour une action précoce.

La conservation, la multiplication et la distribution de matériel génétique approprié, en diversité et en qualité, est une question cruciale pour l'adaptation. Disposer d'une organisation adéquate pour cela est l'un des éléments clés de la capacité d'adaptation. Cela implique de multiples arrangements institutionnels, notamment pour la collecte et la conservation du matériel génétique et des connaissances associées sur ses spécificités (taux de croissance, conditions optimales, etc.) et les façons de l'utiliser (tant pour la production que comme produit, par exemple pour l'alimentation). Cela exige également des règles facilitant l'échange de matériel génétique entre les pays et les institutions publiques et privées qui peuvent le multiplier et le distribuer avec une formation appropriée (Lillesø et al., 2018; Nyoka et al., 2014; Lilles $\emptyset$ et al., 2011) ${ }^{8}$.

La mise en œuvre de mesures d'adaptation pour les forêts, les arbres et l'agroforesterie nécessite généralement une perspective à long terme ainsi qu'une gestion à grande échelle, qui à son tour requiert un environnement favorable qui, dans une large mesure, est en fait le même que celui de la gestion durable des forêts.

La visibilité à long terme des zones destinées à rester des forêts est un élément essentiel de la gestion durable des forêts. Des études ont montré que souvent, les gestionnaires de forêts dans les pays en développement ne considèrent pas l'adaptation comme une priorité ou même comme une préoccupation, simplement parce qu'il y a un risque plus élevé que la forêt cesse d'être une forêt (Guariguata et al., 2012). En cas d'incertitude quant à l'avenir de la forêt, il n'y a aucune incitation à s'adapter, surtout si les mesures visant des bénéfices à long terme induisent des coûts à court terme, que ce soit pour l'investissement ou en raison d'un manque à gagner.

Les droits de propriété, la tenure foncière et les droits d'accès conditionnent les incitations à la gestion durable et à long terme des forêts, des arbres et des ressources qu'ils génèrent (produits ligneux et non ligneux) ainsi que d'autres services écosystémiques. La durée, la stabilité et la protection des droits contre l'empiètement d'autres acteurs revêtent une importance particulière.

Les droits de propriété et d'accès régissant les forêts et les arbres conditionnent également la capacité des populations à en tirer des biens et des

\footnotetext{
6 Pour des informations et des principes généraux ainsi qu'une étude globale des parasites des forêts, voir http://www.fao.org/forestry/pests/94183/en/

7 Voir par exemple pour la Nouvelle-Zélande, les recommandations et les outils disponibles sur https://www.mpi.govt.nz/growing-and-harvesting/forestry/new-zealand-forests-and-the-forest-industry/protecting-nz-forests/ taking-care-of-your-forest/

8 Des informations générales sont disponibles à l'adresse suivante: http://www.fao.org/forestry/seedsmore/en/
} 
revenus et à s'en servir comme tampon. Nombre de ces droits sont coutumiers ou informels et peuvent être menacés lorsque des conventions formalisées sont établies, comme les concessions accordées aux entreprises privées par exemple. Les plus vulnérables, notamment les femmes et les groupes marginalisés, sont souvent les plus dépendants des ressources forestières, et ont également les droits les moins reconnus (qu'ils soient formels ou coutumiers) et les plus difficiles à faire respecter. Par conséquent, lors de l'analyse des dispositions juridiques et des institutions, une attention particulière doit être accordée aux droits d'accès informels, qui constituent souvent un élément clé des stratégies de subsistance des groupes les plus vulnérables.

De nombreuses mesures d'adaptation nécessitent non seulement une gestion à long terme, mais aussi à grande échelle, par exemple en établissant des zones tampons pour réduire l'impact des incendies ou en créant des couloirs pour la biodiversité. Les mesures à long terme et à grande échelle nécessitent des formes de prise de décision et de gestion qui prennent en compte les intérêts et les perspectives de diverses catégories d'acteurs, avec des formes de compensation entre eux.

Les mesures d'adaptation concernant les arbres peuvent être limitées par les difficultés de préparation des plans de gestion forestière, les restrictions d'utilisation d'espèces d'arbres spécifiques, la bureaucratie et la paperasserie liées à la récolte de bois et de produits forestiers non ligneux, la fiscalité élevée et/ou les divers permis nécessaires à la récolte et au transport des produits forestiers. Ces facteurs peuvent entraîner des coûts de transaction très élevés pour les communautés locales dans l'utilisation des arbres et des produits forestiers, et se traduisent par une importance croissante des intermédiaires qui soustraient encore davantage une partie importante des revenus monétaires générés par ces ressources.

Il existe un large éventail d'instruments publics qui peuvent être mis en place pour orienter la gestion forestière, promouvoir la gestion collective et à long terme et compenser les différences de coûts et de bénéfices dans le temps et entre les catégories d'acteurs: lois et règlements, gestion directe ou déléguée des forêts publiques, incitations, y compris les régimes fiscaux, soutien aux instruments fondés sur le marché, souvent en combinaison.

La conception et la mise en œuvre réussies de ces mesures nécessitent l'engagement total de tous les acteurs, car les mesures d'adaptation proposées peuvent modifier l'équilibre entre les objectifs et entre les intérêts (Kolström et al., 2011). Les approches de gestion communautaire sont souvent présentées comme particulièrement efficaces (Keenan, 2015), tout comme l'adaptation basée sur les écosystèmes qui, lorsqu'elle est appliquée efficacement, apporte de la biodiversité et des cobénéfices sociaux, qui renforcent la mise en œuvre des PNA. Par exemple, l'AbE bénéficie de l'engagement actif et de l'inclusion de diverses parties prenantes qui encouragent la gouvernance locale des ressources forestières, l'engagement multisectoriel et la communication intrasectorielle (IIED, 2019). Une fois que les options d'adaptation ont été identifiées, ainsi que les mesures nécessaires pour rendre l'environnement plus favorable, il peut être utile de les classer par ordre de priorité, soit par secteur, soit de manière plus générale. L'annexe 4 donne un aperçu de certaines des approches méthodologiques qui peuvent être utilisées pour ce faire.

\subsection{Compiler et communiquer les perspectives sur les forêts et les arbres}

Le PNA est un processus à long terme qui comprend un suivi et une révision périodique (voir chapitre 7). Il est donc important de suivre le travail effectué, les données recueillies, ainsi que les discussions et les décisions, afin d'évaluer les progrès et d'améliorer le processus. Il est essentiel d'inclure le travail effectué au niveau national ainsi que les informations pertinentes recueillies aux niveaux sous-national et local. Cette compilation systématique pourrait apporter une contribution utile à la préparation d'informations spécifiques pour les différentes catégories de parties prenantes (voir chapitre 8).

L'une des principales exigences du processus du PNA est de veiller à ce que ses parties prenantes soient conscientes des spécificités et des défis rencontrés par le secteur forestier, et qu'elles 
soient informées des développements au fur et à mesure de l'évolution du processus. Pour ce faire, il convient de rédiger un bref document résumant les principales conclusions et recommandations concernant les forêts et les arbres, qui puisse être utilisé pour fournir un retour d'information à toutes les parties prenantes concernées, pour préparer du matériel de communication dans le secteur et pour servir de référence à du matériel plus ad hoc pour des activités sectorielles spécifiques. Ce document résumera les informations recueillies au cours des étapes 5.1 à 6.4, y compris la méthodologie utilisée, les données et les sources. Les documents et informations existants peuvent être rassemblés dans un répertoire organisé et régulièrement mis à jour, facilement accessible à toutes les parties prenantes. En outre, une contribution spécifique au processus du PNA devrait être préparée, sur la base de ces documents de référence, ainsi que des contributions ciblées sur plusieurs groupes ou sections, en fonction de la manière dont le travail a été organisé.

Étant donné les liens des forêts, des arbres et de l'agroforesterie avec de multiples secteurs aux niveaux national et local, il est essentiel d'organiser des canaux de communication efficaces avec eux. Cette communication prendra différentes formes, en fonction de l'organisation du processus aux niveaux national et local. Elle doit être en phase avec les différentes étapes du processus afin de fournir des informations appropriées en temps utile. Elle peut commencer par l'élaboration d'un document de synthèse résumant les résultats du bilan, décrivant le secteur et sa contribution à l'économie, à l'emploi et aux moyens d'existence aux niveaux national et local, en mettant l'accent sur les populations vulnérables ainsi que sur ses perspectives de développement. L'accent sera également mis sur les vulnérabilités du secteur au changement climatique, ses contributions potentielles à l'adaptation d'autres secteurs et ses moyens d'adaptation potentiels (voir le bilan au point 5.1 et l'annexe 2). Lors de l'élaboration du PNA , des documents plus ciblés pourraient être préparés pour les acteurs d'un autre secteur spécifique, répondant à leurs propres besoins et/ ou soulignant la manière dont le secteur pourrait contribuer à l'adaptation des forêts, des arbres et de l'agroforesterie (voir les tableaux de la section 6.3). Par exemple, il pourrait y avoir un document spécifique sur les interactions entre les cultures, le bétail et les arbres, mettant en évidence les contributions des forêts, des arbres et de l'agroforesterie à la production végétale et animale, les contributions potentielles pour soutenir leur adaptation et ce qui est nécessaire pour faciliter ces contributions en termes de tenure foncière et des arbres. Ces documents peuvent comprendre l'identification de mesures précises, de leurs coûts et avantages et des ressources nécessaires à leur mise en œuvre. Ces documents de communication devraient également souligner les avantages de l'adaptation pour le développement durable, y compris les cobénéfices de l'atténuation (voir section 5.3). La communication de ces perspectives se fera à la fois de manière formelle et informelle par le biais des échanges réguliers établis dans le cadre du processus, avec d'autres secteurs, tant au niveau national, sous-national que local.

\subsection{Examiner l'intégration de l'adaptation au changement climatique dans les stratégies, programmes et plans forestiers nationaux et infranationaux}

Cette section vise à analyser comment l'adaptation au changement climatique peut être mieux intégrée dans les stratégies actuelles et futures ainsi que dans les plans aux niveaux national et infranational (voir section 5.1). Cela nécessite une compréhension approfondie des dispositions et des cycles institutionnels pertinents ainsi que l'engagement de divers acteurs gouvernementaux et non gouvernementaux. Cet aspect de l'adaptation au changement climatique est particulièrement important pour les forêts et les arbres, étant donné que les compétences pour les différents secteurs et les questions qui s'y rapportent sont généralement partagées entre diverses entités nationales et infranationales: ministères de tutelle, entités publiques spécifiques, gouvernements locaux; avec une répartition des compétences qui varie selon les secteurs et les questions. 
Étant donné l'étendue des secteurs et des niveaux de gouvernement qui peuvent influencer la gestion des forêts, nous proposons de réaliser cette évaluation de manière organisée.

Au niveau national:

- d'abord, examiner les stratégies, programmes et plans forestiers au niveau national;

- ensuite, examiner d'autres documents nationaux généraux et sectoriels qui pourraient influencer les forêts: stratégies et plans de développement, plans d'aménagement du territoire, eau, agriculture, sécurité alimentaire;

- se référer aux stratégies, programmes et plans forestiers au niveau national pour examiner comment ils pourraient être modifiés afin d'intégrer les objectifs des stratégies et plans nationaux plus généraux, ceux des stratégies et plans nationaux sectoriels en ce qui concerne les forêts, les arbres et l'agroforesterie;

- y intégrer ce qui doit être fait au niveau national pour l'adaptation des forêts, des arbres et de l'agroforesterie et pour renforcer leur contribution à l'adaptation d'autres secteurs;

- préparer des lignes directrices en vue d'un examen aux niveaux infranational et local, selon les principes ci-dessous.

Au niveau infranational et local et pour tous les types de forêts et les objectifs de gestion:

- d'abord, examiner les stratégies, programmes et plans forestiers au niveau infranational (par exemple provincial);

- ensuite, examiner d'autres documents généraux et sectoriels infranationaux susceptibles d'influencer les forêts: stratégies, programmes et plans de développement, plans d'aménagement du territoire, eau, agriculture, sécurité alimentaire;

- se référer aux stratégies et plans forestiers au niveau infranational pour examiner comment ils devraient être modifiés afin d'intégrer les objectifs des stratégies et plans nationaux plus larges, les stratégies et plans sectoriels infranationaux en ce qui concerne les forêts, les arbres et l'agroforesterie;

- y intégrer ce qui doit être fait au niveau national pour l'adaptation des forêts, des arbres et de l'agroforesterie et pour renforcer leur contribution à l'adaptation d'autres secteurs;

- élaborer des directives en vue d'un examen aux niveaux infranational et local, selon les principes ci-dessous.

Les évaluations aux niveaux infranational et local peuvent identifier les questions à traiter à un niveau plus large.

Le Conseil canadien des Ministres des Forêts (CCMF) a soigneusement mis en œuvre l'intégration du changement climatique dans les stratégies, programmes et plans forestiers. En fait, il a reconnu que le changement climatique posera des défis (en termes d'atteinte des objectifs de gestion durable des forêts (GDF) et, dans certains cas, apportera des opportunités au secteur forestier canadien grâce à l'amélioration de la productivité des forêts (Johnston et al., 2009). Il a donc recommandé que la prise en compte du changement climatique et de la variabilité climatique future soit intégrée dans tous les aspects de la GDF au Canada (CCMF, 2008). À la suite de cette recommandation, le Groupe de travail sur le changement climatique (CCTF) a été créé avec pour mission de recueillir des informations pertinentes et de développer des outils pour aider les gestionnaires et les professionnels des ressources forestières de tout le pays à déterminer la meilleure façon d'intégrer les considérations relatives au changement climatique dans les plans, les pratiques et les politiques de GDF. Parmi les principaux produits élaborés par le groupe de travail figurent un cadre d'adaptation (Williamson et al., 2012), un guide d'évaluation de la vulnérabilité (Edwards et al., 2015) et d'autres rapports techniques faciles à comprendre qui appuient le cadre et le guide.

En outre, le PNA peut être un vecteur principal de mise en œuvre pour la CDN (voir encadré 11). 
ENGADRÉ 11.

\section{Établir des liens entre les Plans nationaux d'adaptation (PNA) et les contributions déterminées au niveau national (CDN) pour améliorer la planification de l'adaptation pour le secteur de l'agriculture au Viet Nam}

La CDN du Viet nam considère l'agriculture comme un secteur prioritaire pour l'élaboration de stratégies de lutte contre le changement climatique. Le secteur agricole a également été mis en évidence dans le processus d'élaboration du PNA au Viet Nam, avec un accent mis sur la réduction des vulnérabilités du secteur agricole au changement climatique et qui s'est traduit par une intégration de l'adaptation dans la planification du développement agricole à tous les niveaux, à moyen et long terme.

Si les CDN constituent les engagements intégrés des pays en matière d'atténuation et d'adaptation au niveau stratégique et que les PNA permettent l'élaboration et la mise en œuvre des priorités en matière d'adaptation au niveau opérationnel, il est de plus en plus reconnu que, malgré leurs caractéristiques distinctives, ces instruments de planification face au changement climatique doivent être reliés, séquencés et alignés pour favoriser l'établissement de liens et stimuler l'action en faveur du climat. Un tel alignement entre le processus du PNA du Viet Nam et la révision de sa CDN en vue de sa soumission la CCNUCC à l'horizon 2020 offre une excellente occasion d'exploiter les liens, de renforcer l'intégration des objectifs d'adaptation des instruments de planification face au changement climatique et de développer des stratégies intégrées de lutte contre ce phénomène.

En conséquence, le programme PNA -Ag du Viet nam a été choisi pour fournir un soutien au Ministère de l'agriculture et du développement rural (MARD) à travers la définition de pistes de réflexion permettant d'harmoniser l'élaboration de stratégies d'adaptation efficaces dans le cadre du processus du PNA et en vue d'une utilisation des contributions dans la révision et la mise à jour de la CDN. Les étapes suivantes ont contribué à l'établissement d'une base solide de preuves pour faciliter l'alignement de la révision de la CDN sur le processus du PNA.

1. Un inventaire des pratiques d'adaptation au changement climatique. Un vaste exercice d'inventaire a été mené sur 124 projets structurels d'adaptation au changement climatique (par exemple, le développement des infrastructures et l'amélioration des systèmes d'irrigation) et 907 projets non structurels d'adaptation au changement climatique (par exemple, le développement de nouvelles variétés de cultures) dans l'agriculture. L'inventaire a démontré le déséquilibre entre ces mesures, plus de 63 pour cent des fonds étant alloués à l'amélioration de la résilience des grands projets d'infrastructure. Cela a conduit à la recommandation du PNA -Ag selon laquelle ces deux types de mesures devraient être intégrés pour parvenir à un développement durable à faible émission de carbone. Une analyse des insuffisances s'est ajoutée à l'inventaire en identifiant le manque de cohérence entre le changement climatique et les politiques agricoles, l'absence de mécanismes financiers permettant de transposer à plus grande échelle les modèles/programmes pilotes réussis, le manque de capacité technique à appréhender les risques ainsi que la participation modeste du secteur privé.

2. Une évaluation de la vulnérabilité et du risque climatique. Au cours du processus d'élaboration de la $\mathrm{CDN}$, les décideurs politiques ont identifié le manque de données climatiques cohérentes comme un obstacle à la priorisation des programmes et 
à la prise de décisions fondée sur l'évaluation des risques. Par la suite, le PNUD a développé une plateforme de connaissances open-source à travers la création du site Viet Nam Climate Risk website (site web sur les risques climatiques au Viet Nam) (PNUD, 2018). Les indices de risques climatiques ont été élaborés en combinant de nombreuses sources de données telles que des profils sur le changement climatique et les risques associés, des indices de vulnérabilité et des statistiques socioéconomiques. Sous les auspices du MARD, des discussions ont eu lieu entre experts nationaux et internationaux pour la sélection des indicateurs et l'attribution de pondérations à la vulnérabilité. L'un des principaux résultats de cette activité a été l'élaboration d'une méthodologie commune pour évaluer la vulnérabilité de quatre secteurs agricoles majeurs considérés comme prioritaires par le MARD: les cultures, l'élevage, l'aquaculture et les infrastructures d'irrigation.

3. Une évaluation des pertes et des dommages dans l'agriculture. Les pertes et dommages sont définis comme des impacts du changement climatique qui dépassent les limites de l'adaptation. Il est essentiel de veiller à ce que les effets durables du changement climatique soient pris en compte dans les plans nationaux d'adaptation pour la gestion des risques et la réalisation d'investissements d'adaptation rentables. L'évaluation a révélé que le changement climatique, à l'origine de l'élévation du niveau de la mer et de la sécheresse, constitue une grave menace pour le secteur agricole au Viet Nam. En l'absence de mesures d'adaptation, une élévation du niveau de la mer de $22 \mathrm{~cm}$ réduirait les terres cultivables d'un million d'hectares par rapport à une situation de référence de 2004, et réduirait considérablement la production de riz dans le delta du Mékong, tandis qu'une élévation du niveau de la mer d'un mètre risquerait d'inonder près d'un tiers du réseau ferroviaire vietnamien. En outre, l'évaluation a souligné que le secteur des assurances est susceptible de jouer un rôle majeur au Viet Nam, car il fournirait de plus en plus une couverture partielle pour les pertes et les dommages inévitables. Les recommandations basées sur cette étude soulignent qu'à mesure que le secteur agricole se développe, il est crucial d'orienter ces investissements vers une économie plus résiliente, par exemple dans des infrastructures résistantes aux changements climatiques.

Outre les informations détaillées qui ont été recueillies, ces activités d'alignement ont également permis de tirer des enseignements essentiels pour la base de connaissances qui alimentera le volet «adaptation» de la révision et de la mise à jour de la CDN et qui servira également de base aux travaux ultérieurs d'élaboration du PNA du Viet Nam. Ces activités comprennent:

- les efforts en cours pour renforcer les capacités de planification de l'adaptation, accroître l'efficacité et optimiser l'affectation des ressources;

- L'alignement entre les PNA et les CDN peut être amélioré grâce à une collaboration formelle et informelle;

- La coordination et la relation institutionnelles entre le PNA-Ag et la mise à jour de la CDN ont permis d'accroître la cohérence entre les cadres politiques;

- L'approche « test et essai » utilisée dans le secteur agricole peut éclairer la planification globale de l'adaptation;

- La gestion de l'information et l'évaluation des risques pour la planification de l'adaptation nécessiteront des investissements plus importants. 


\section{4}

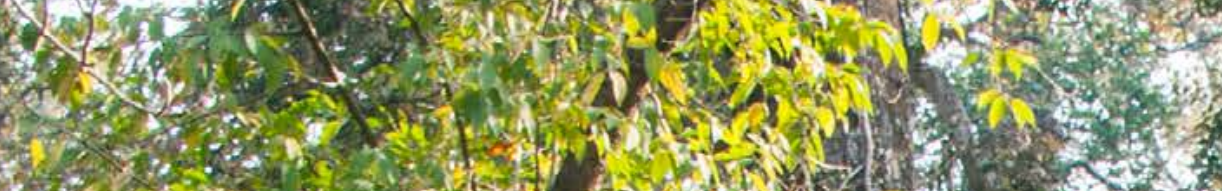

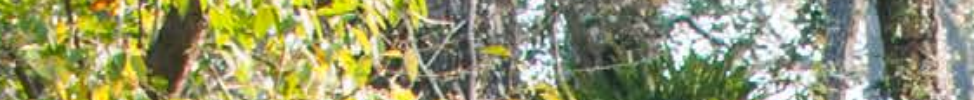

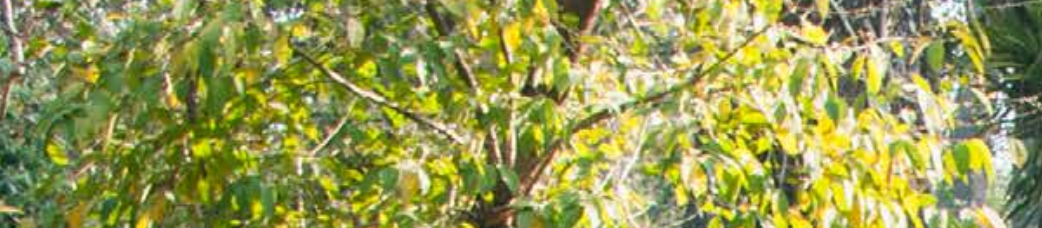

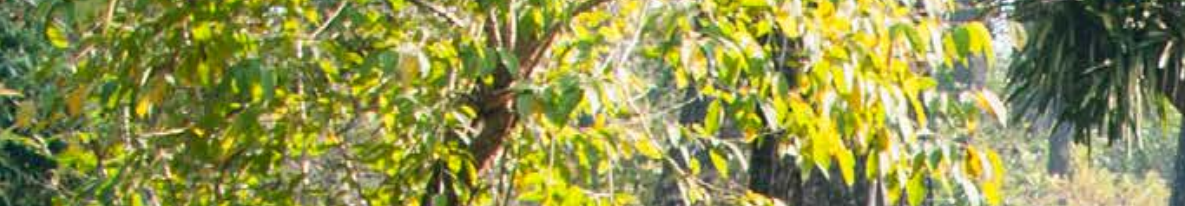

in

$\left.3 x^{2}\right)$

$4)^{2}$

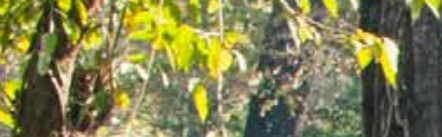

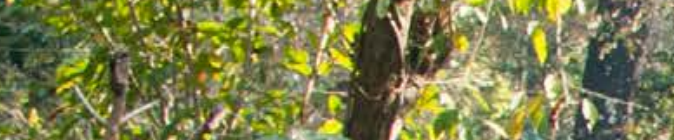

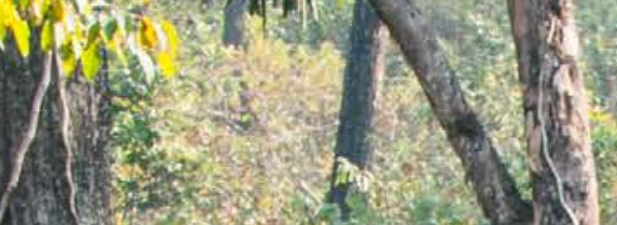

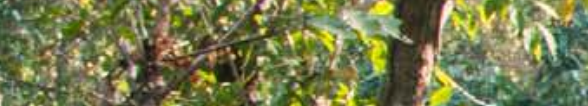

(1)

(1)

$3.5)$ (2)

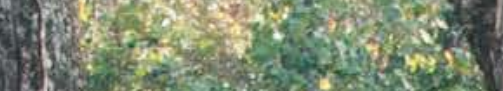

if

(2) (6)

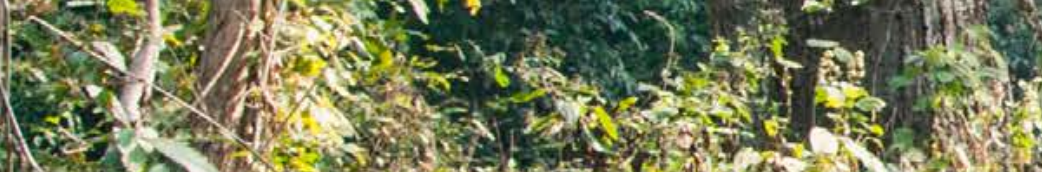
a

1.5. (5)

(6.5. (1)

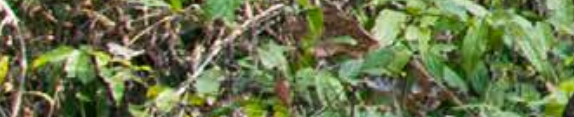

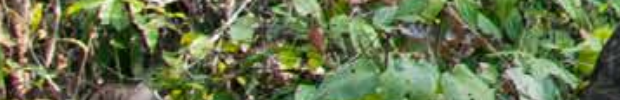

int (2)

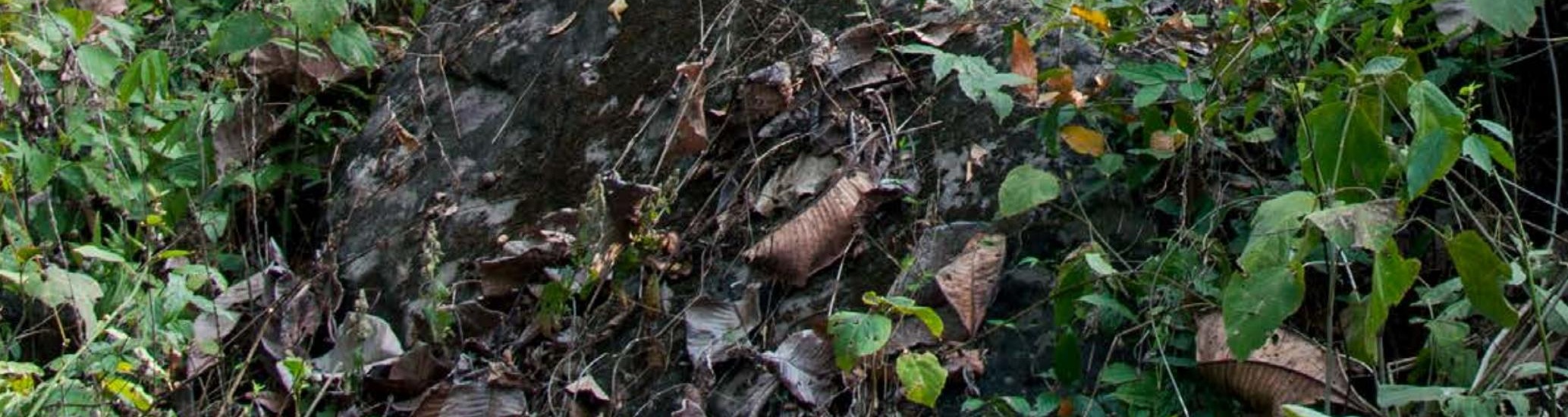

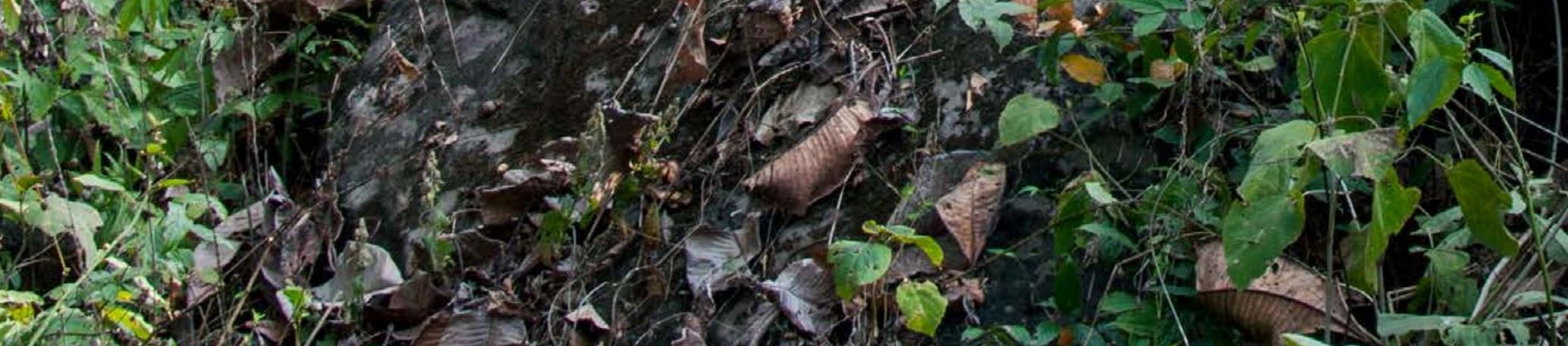

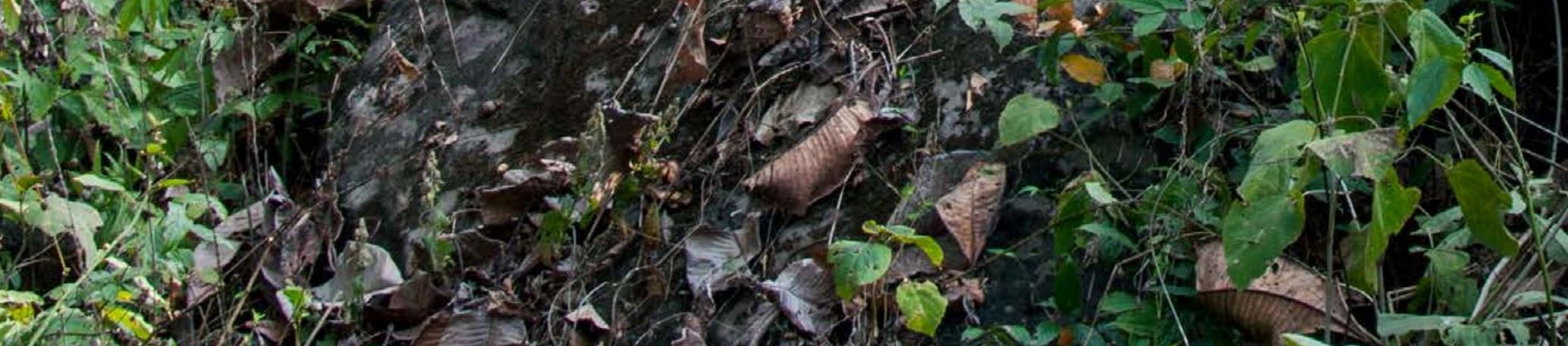

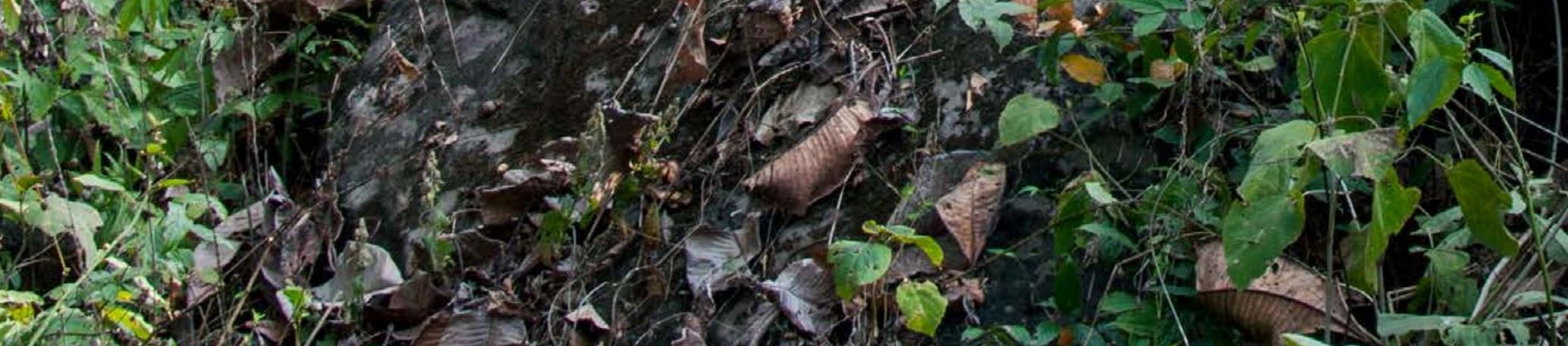

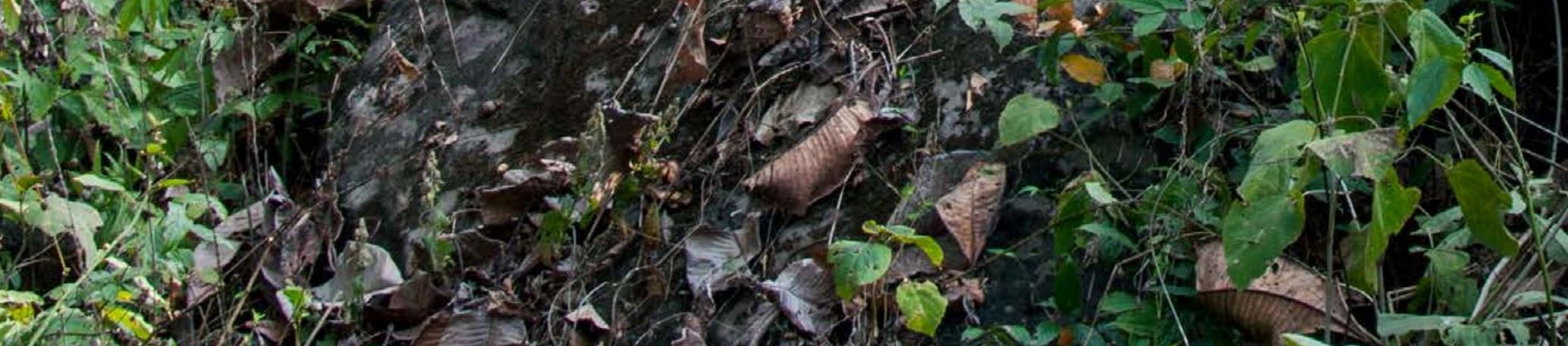
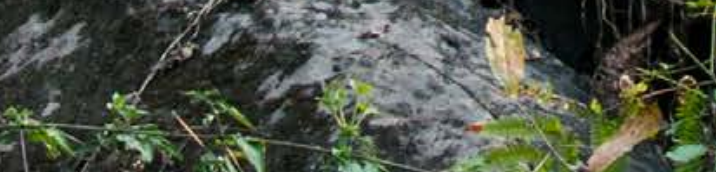
in:

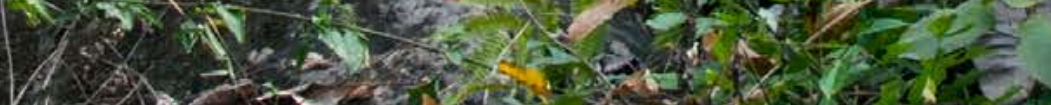
1 



\section{- Stratégies de mise en œuvre}

\author{
Ce chapitre a pour \\ objet de veiller à \\ ce que les forêts, \\ les arbres et \\ l'agroforesterie \\ soient correctement \\ intégrés dans la \\ mise en œuvre \\ du PNA avec \\ les moyens \\ institutionnels, \\ humains et \\ financiers \\ appropriés, ce qui \\ correspond au point \\ $C$ des orientations \\ techniques du PNA.
}

\subsection{Accorder aux forêts et aux arbres la priorité requise dans le PNA}

Le processus du PNA identifie souvent des domaines d'action prioritaires ou même des activités prioritaires, dont la portée et la nature dépendent de l'organisation du processus lui-même (par exemple régional ou par secteurs) ainsi que des critères et procédures utilisés pour établir les priorités. À cet égard, il est important de souligner trois points afin d'intégrer de manière appropriée les forêts et les arbres: (i) l'adaptation des forêts et des arbres afin qu'ils puissent remplir leurs rôles économiques, sociaux et environnementaux, y compris leur contribution à l'atténuation, (ii) la contribution essentielle que les forêts et les arbres peuvent apporter à l'adaptation en général, (iii) la nécessité d'agir maintenant, non pas en dépit du temps nécessaire à la Foresterie, mais précisément à cause de celle-ci.

Ces trois points devront être reflétés de trois manières différentes. D'abord, en consacrant une section à l'adaptation des forêts, des arbres et de l'agroforesterie, à travers la prise en compte des besoins du secteur et de ce qui est requis pour sa contribution à l'adaptation d'autres systèmes/secteurs. Ensuite, en faisant en sorte que les contributions potentielles des forêts et des arbres à l'adaptation d'autres secteurs soient décrites de manière adéquate dans les sections appropriées du document, en se référant, le cas échéant, à la section consacrée aux forêts et aux arbres, par exemple, en ce qui concerne les spécificités techniques. Enfin, en adoptant une perspective à long terme avec une stratégie de planification adéquate couvrant non seulement les mesures techniques simples mais également les questions structurelles qui déterminent un environnement favorable nécessaire pour que les acteurs s'adaptent et investissent, comme la stabilité des zones forestières et la sécurité de la propriété foncière et arboricole.

\section{RÉPUBLIQUE-UNIE DE TANZANIE}

Vue sur les rizières du Kiroka.

(CFAO/Daniel Hayduk 


\subsection{Mettre en place les moyens de permettre un changement transformationnel}

L'adaptation au changement climatique et la garantie de la résilience de l'économie, des populations et des écosystèmes anthropisés au changement climatique nécessiteront non seulement un ensemble de réponses techniques, mais aussi de profonds changements systémiques au sein de l'économie et dans la relation entre l'économie et la nature. Ces changements transformationnels nécessiteront un environnement global favorable à l'adaptation et un alignement d'un large éventail de politiques et de moyens de mise en œuvre, à long terme. Les objectifs d'adaptation devront être articulés avec d'autres stratégies telles que la conservation de la biodiversité, le développement économique... aux niveaux national et infranational (voir 7.5) ainsi que la mobilisation de ressources supplémentaires, y compris le financement du climat (voir 7.3). Cela peut être facilité par l'élaboration d'une stratégie d'adaptation à long terme, approuvée au plus haut niveau. Une telle stratégie ne doit pas être un document isolé. L'objectif d'une stratégie à long terme est d'assurer la cohérence dans le déploiement des activités d'adaptation en fournissant une orientation générale et en énumérant les modifications concrètes à apporter aux règles, politiques et mesures.

La mise en œuvre de mesures d'adaptation par les gestionnaires de forêts, ou les communautés forestières, les agriculteurs et autres acteurs nécessite souvent la coordination de différentes institutions et acteurs de soutien ainsi que des conditions favorables (voir en particulier la section 6.5). En outre, la mise en œuvre des mesures d'adaptation pour les forêts, les arbres et l'agroforesterie nécessite généralement une perspective à long terme et une gestion à grande échelle.

Par conséquent, il ne suffit pas d'identifier les options et les mesures d'adaptation liées aux secteurs forestier et arboricole et leur rôle pour l'adaptation: il faut également examiner ce qui est nécessaire pour faciliter leur mise en œuvre ainsi que le rôle des différents acteurs, y compris les conditions favorables. Cette analyse doit être effectuée en tenant compte du bilan sectoriel et général réalisé précédemment, en particulier les lois, règlements et dispositions institutionnels existants ainsi que l'environnement économique et social (voir section 5.1). La capacité des institutions et règles existantes à rendre possible (ou non) une action collective et à long terme est un point essentiel à examiner.

L'agroforesterie et l'intégration des arbres dans les systèmes agricoles sont un exemple pertinent de l'importance cruciale d'un environnement propice approprié. De nombreux CDN et PNA encouragent l'agroforesterie mais il existe souvent des contraintes à son développement. L'élaboration d'une politique agroforestière par le gouvernement du Népal (voir encadré 12) montre comment ces contraintes peuvent être identifiées et prises en charge. La plupart des PNA publiés comprennent des recommandations pour la plantation d'arbres à des fins d'adaptation diverses. Toutefois, les mesures relatives à l'environnement favorable nécessaire à la plantation d'arbres, comme les systèmes de tenure foncière, de semences et de plants, font souvent défaut, en particulier pour les pays n'ayant pas de tradition établie de plantation de forêts.

\section{ENGADRÉ 12.}

\section{Le développement d'une politique agroforestière au Népal}

L'élaboration d'une politique agroforestière nationale est en accord avec la CDN (2016) et la politique du Népal en matière de changement climatique (2011) qui identifient les forêts et les arbres comme étant extrêmement importants pour promouvoir à la fois l'adaptation au changement climatique et l'atténuation, y compris l'agroforesterie. Elle a été soutenue par le Climate Technology Centre and Network (CTCN) en collaboration avec le Centre Mondial d'Agroforesterie (ICRAF). 
En 2016, le gouvernement du Népal a mis en place un comité de coordination interministériel (IMCC) composé du Ministère du développement agricole (MoAD), du Ministère de la conservation des forêts et des sols (MFSC), du Ministère de la population et de l'environnement (MOPE), de l'Autorité Nationale Désignée (AND) et de l'ICRAF pour coordonner et fournir un soutien global à l'élaboration des politiques. L'IMCC a élaboré et approuvé une feuille de route pour l'élaboration d'une politique nationale d'agroforesterie. Une analyse de 30 politiques et lois pertinentes existantes affectant l'agroforesterie au Népal a été réalisée. Trois ateliers régionaux ont été organisés dans les zones de haute, moyenne et basse montagne du Népal, avec des représentants des ministères et entités gouvernementaux, des instituts de recherche et des universités, des agriculteurs, des associations et coopératives d'agriculteurs, des ONG et des ONGI, et des prestataires de services. Ces ateliers ont fourni des informations essentielles sur les contraintes liées à l'adoption de l'agroforesterie, le soutien actuellement disponible pour les agriculteurs par le biais de divers acteurs, et les attentes des agriculteurs par rapport à une politique agroforestière. Une visite d'étude des membres du CCMI a été organisée en Inde, le seul pays ayant approuvé une politique agroforestière nationale et qui la met actuellement en œuvre.

Les membres de l'IMCC ont eu des échanges avec des hauts fonctionnaires et des décideurs politiques indiens qui ont activement contribué à l'élaboration de la politique agroforestière indienne et qui participent à sa mise en œuvre.

Les informations générées par l'examen des politiques gouvernementales existantes et les contributions des ateliers régionaux ont servi de base à l'élaboration de l'avantprojet de la politique agroforestière. Cet avant-projet a été examiné et modifié par l'IMCC, puis partagé avec les parties prenantes au cours de trois ateliers nationaux. Les contributions reçues au cours de ces ateliers ont été intégrées par l'IMCC dans le projet de politique, après quoi le document a été révisé par les ministères de tutelle qui ont fourni plus de suggestions pour améliorer davantage la politique. En septembre 2018, les projets en version anglaise et népalais ont été soumis à l'IMCC pour un traitement final à travers le mécanisme gouvernemental.

La politique nationale d'agroforesterie adoptée en 2019 s'attaque à certaines des principales contraintes identifiées, notamment par la reconnaissance des systèmes agroforestiers, la facilitation du transport et de la vente des produits forestiers issus de la pratique de l'agroforesterie, les dispositions visant à faciliter les prêts et les assurances pour les agroforêts, la mise à l'essai des systèmes agroforestiers, la réduction des conflits entre l'homme et la faune, et la formation et l'accompagnement en matière d'agroforesterie.

Sources: https://www.ctc-n.org/news/ctcn-nepal-developing-national-agroforestry-policy; http://www.worldagroforestry.org/project/technical-support-formulate-national-agroforestry-policy-nepal https://familyforestnepal.com/news_post/national-agro-forest-policy-2076/

La conception de stratégies d'adaptation pour les cultures de produits arboricoles tels que le café, le cacao et le caoutchouc, fournit un autre bon exemple de la manière dont un environnement favorable peut être essentiel pour soutenir un changement à grande échelle des variétés plantées et/ou l'adoption de nouvelles pratiques d'arboriculture, telles que l'introduction d'arbres d'ombrage. De tels changements nécessiteront des investissements initiaux et pourraient à court terme, réduire les revenus tirés de l'agriculture. D'autre part, étant donné que dans de nombreux pays, les plantations vieillissent et, dans certains cas, connaissent une baisse de rendement, une opportunité de remplacer les arbres pourrait se présenter. Il faudrait pour cela, mettre à disposition le germoplasme et les connaissances approprié, concevoir des voies de transition 
techniques qui soient économiquement réalisables pour les petits exploitants et fournir un soutien financier approprié de la part d'autres acteurs de la chaîne de valeur et/ou du secteur public, au niveau infranational, national ou international.

Les lacunes en matière de création d'un environnement favorable doivent être correctement identifiées et prises en charge: c'est-à-dire qu'est-ce qui doit être fait? par qui? et quelle synergie devrait exister entre les différentes actions. Tout d'abord, les lois sur la foresterie peuvent nécessiter des amendements qui devront à leur tour être mises en œuvre par les autorités infranationales afin de garantir la propriété à long terme des terres et/ou des arbres de sorte que les acteurs privés, tels que les gestionnaires de forêts et les agriculteurs, puissent investir dans des mesures d'adaptation. Deuxièmement, il convient d'établir un calendrier de mise en œuvre, en commençant éventuellement par les mesures les plus faciles à mettre en œuvre afin de montrer les progrès rapides tout en initiant des changements structurels plus larges. Enfin, la stratégie comprendrait des objectifs généraux et une série d'étapes organisées de manière progressive et cohérente, avec un calendrier et des examens réguliers des progrès réalisés (voir chapitre 8 ).

\subsection{Mobiliser des ressources financières}

Cette section examine les ressources financières qui peuvent être orientées, ou réorientées, vers les objectifs d'une stratégie à long terme pour l'adaptation des forêts et des arbres au changement climatique. Ces ressources comprennent les ressources publiques nationales, les investissements du secteur privé (nationaux et internationaux) et l'aide publique internationale. Cette aide publique internationale comprend l'ODA, le financement des banques de développement régionales et internationales, les mécanismes spécialisés tels que le Fonds pour l'environnement mondial (FVC) et le financement du climat (Fonds vert pour le climat, Fonds d'adaptation).

Une étape préliminaire utile pour plaider en faveur de ressources financières adéquates pour les forêts et les arbres consiste à quantifier la contribution du secteur à l'économie en utilisant des éléments de l'inventaire (section 5.1) et en recueillant une estimation convaincante des coûts et avantages des mesures d'adaptation, y compris les avantages indirects pour d'autres secteurs.

Une première catégorie de ressources financières est constituée de celles qui sont consacrées directement au secteur forestier par les acteurs publics, le gouvernement national, les agences spécifiques, les autorités infranationales, soit comme investissement direct, soit pour orienter les investissements et les pratiques privés. Il convient de noter que ces ressources sont probablement insuffisantes pour couvrir l'ensemble des besoins d'adaptation, en particulier dans de nombreux pays en développement où le secteur forestier est déjà gravement sous-financé, sans même tenir compte des besoins liés au changement climatique.

Outre un financement accru, les règles mêmes qui régissent l'attribution sectorielle des ressources (et leur utilisation par le secteur) devront souvent être modifiées afin de faciliter ou d'intensifier la mise en œuvre des mesures d'adaptation dans les secteurs de la Foresterie, de l'agroforesterie et des systèmes arboricoles. Par exemple, la mobilisation de ressources pour l'agroforesterie et l'intégration des arbres dans les systèmes agricoles et les paysages pourrait nécessiter d'apporter certaines modifications aux conditions d'attribution et aux règles relatives à la fourniture d'un appui à la Foresterie, aux cultures et à l'élevage. Un autre exemple concerne la recherche de mesures axées sur l'atténuation. La plupart des CDN contiennent d'importants engagements visant à accroître la contribution des forêts à l'atténuation. Puisqu'en effet, l'atténuation basée sur les forêts et les arbres n'aura lieu que si les forêts et les arbres se sont correctement adaptés au changement climatique, les mesures d'adaptation - et le financement pourraient être intégrés dans les politiques et les mesures axées sur les engagements en matière d'atténuation. Les considérations d'adaptation doivent être intégrées dans les mesures et les projets spécifiquement axés sur l'atténuation.

Une deuxième catégorie de ressources pourrait être trouvée en promouvant les AbE (solutions basées sur la nature) et en réorientant en conséquence vers la foresterie certaines des ressources affectées par les acteurs publics, le gouvernement local, les agences spécifiques, 
les autorités infranationales, vers des secteurs qui bénéficieront des actions dans le secteur forestier, soit pour leur propre adaptation, soit pour d'autres avantages de développement comme l'eau, l'énergie, les villes et les établissements humains, les infrastructures, la biodiversité et la santé. Les acteurs privés de ces secteurs peuvent également être encouragés ou incités par les politiques et mesures publiques à financer des activités forestières. La mobilisation de l'ODA, le financement des banques régionales et internationales de développement, les mécanismes dédiés tels que le FEM et le financement du climat peuvent être soutenus en mettant l'accent, le cas échéant, sur les multiples avantages à long terme de mesures spécifiques non seulement pour l'adaptation mais également pour l'atténuation et les divers objectifs de développement durable.

Nombre de ces fonds, mécanismes et donateurs internationaux ont des objectifs assez spécialisés, ce qui rend souvent difficile la promotion de projets à buts multiples. Cette spécialisation peut conduire les fonds ou les donateurs à ne pas sélectionner un projet parce qu'il n'est pas assez performant par rapport à leur propre objectif spécifique. Il y a cependant des évolutions à cet égard, avec une plus grande réceptivité à des objectifs divers, motivée à la fois par les ODD et par la rareté des financements par rapport aux besoins. Ici, on peut essayer de tirer parti du fait que les projets d'adaptation liés à la foresterie présentent souvent de multiples avantages (par exemple, des écosystèmes sains, la réduction des risques d'incendie, la conservation de la faune et de la flore, etc.), qui peuvent être stratégiquement mis en avant en fonction de l'objectif principal du fonds ou de l'intérêt du donateur. Il pourrait donc y avoir de nouvelles opportunités pour la foresterie, à condition que les avantages soient bien articulés et quantifiés.

Le Fonds vert pour le climat (FVC) doit consacrer la moitié de ses ressources à des projets d'adaptation. Il a deux domaines de résultats particulièrement pertinents pour les forêts: les forêts et l'utilisation des terres (dans le volet atténuation) et les écosystèmes (dans le volet adaptation). Cependant, le FVC les examine désormais ensemble dans le cadre de projets transversaux d'atténuation et d'adaptation. Ces résultats seront publiés dans les prochains guides sectoriels en cours d'élaboration par le FVC. Comme le souligne le récent rapport spécial du FVC sur le changement climatique et les terres, les avantages en matière d'adaptation, d'atténuation et de développement durable devront être pris en compte de manière holistique lorsqu'il s'agira d'envisager des mesures basées sur les terres, et cette intégration est également un moyen de parvenir à un bon rapport coûtefficacité. Le Programme de préparation et de soutien préparatoire du FVC, en particulier, offre un soutien aux pays pour le renforcement de leurs capacités institutionnelles, leurs mécanismes de gouvernance et leurs cadres de planification et de programmation, ce qui peut jeter les bases de projets transformationnels à plus grande échelle.

Enfin, de nouvelles formes de financement peuvent être mobilisées pour l'adaptation, en particulier si l'on considère le volet productif et économique des mesures d'adaptation: le financement mixte, c'est-à-dire l'utilisation stratégique du capital de développement public ou philanthropique pour favoriser la mobilisation de financements commerciaux privés; les obligations vertes, une forme d'instrument de dette reliant les fonds générés à des investissements respectueux du climat ou de l'environnement; et le crowdfunding, qui consiste à mettre en commun de petites quantités de capitaux provenant d'un nombre potentiellement important de bailleurs de fonds intéressés (Louman et al., 2020).

\subsection{Améliorer la capacité de planification et de mise en œuvre de l'adaptation pour les forêts et les systèmes arboricoles}

Les perspectives et la planification à long terme sont indispensables pour les forêts, les arbres et l'adaptation de l'agroforesterie. Dans certains pays, la planification à long terme des activités forestières existe déjà souvent avec plusieurs niveaux de planification, jusqu'à l'unité de gestion forestière. Dans de tels cas, l'adaptation peut être assez facilement intégrée comme un facteur supplémentaire à prendre en compte, dans le cadre de la gestion durable des forêts et des programmes 
forestiers nationaux des pays. Lorsque ces instruments de planification n'existent pas ou ne sont pas largement utilisés, ils doivent être créés et promus dans le cadre de la stratégie d'adaptation. Les initiatives de renforcement des capacités devront tenir compte de la particularité du secteur forestier et de son interrelation systémique avec toute une série d'autres secteurs (voir section 4). Il est clair que l'adaptation doit être cohérente avec les plans des secteurs avec lesquels ils interagissent: cultures et élevage, aménagement du territoire et développement urbain, biodiversité, eau et énergie. Une telle approche intégrée est mieux mise en œuvre au niveau du paysage, où les synergies et les compromis doivent être abordés.

Des capacités de planification et notamment de planification 《intégrée » seront donc nécessaires pour les pouvoirs publics, à différents niveaux, ainsi que pour les gestionnaires de forêts et les acteurs impliqués dans la préparation des plans aux différents niveaux indiqués. Les initiatives de renforcement des capacités doivent donc répondre aux besoins de ces acteurs.

\subsection{Promouvoir la coordination et la synergie aux niveaux national et infranational}

\author{
Le PNA doit également examiner les différents \\ moyens qui peuvent être mobilisés pour \\ promouvoir la coordination entre les différents \\ niveaux, du local au national, entre les secteurs et \\ entre les acteurs. Cet aspect est particulièrement \\ important pour les forêts, les arbres et \\ l'agroforesterie, étant donné que les compétences \\ pour les différents secteurs et les questions qui \\ s'y rapportent sont généralement partagées entre \\ diverses entités nationales et infranationales \\ telles que: les ministères de tutelle, les entités \\ publiques spécifiques, les gouvernements locaux; \\ avec une répartition des compétences qui varie \\ selon les secteurs et les sujets.
}

Le processus du PNA lui-même, comme discuté au chapitre 4, présente une bonne opportunité pour initier ou renforcer les liens entre les niveaux et les secteurs. Les efforts de coordination doivent être menés à la fois entre les niveaux, au sein du secteur forestier, et entre les secteurs à chaque niveau, en tenant compte des différences organisationnelles entre les secteurs. Deux niveaux revêtent une importance particulière, notamment parce qu'ils sont communs à la plupart des questions: le niveau national, entre les ministères compétents, et au niveau du paysage, en mobilisant les institutions et entités publiques et privées qui opèrent sur le terrain, car c'est là que la plupart des questions interagissent et doivent être prises en compte conjointement (voir encadré 13).

\section{ENCADRÉ 13.}

Intégration des approches paysagères dans le processus de planification de l'adaptation aux Philippines

Les Philippines ont été pionnières dans la réponse au changement climatique avec la création du Comité inter-agences sur le changement climatique dès 1991, en raison des risques persistants et de nombreuses catastrophes auxquels elles ont été confrontées et qui sont causés par le climat ou qui lui sont liées. Aujourd'hui, son successeur - le Comité sur le changement climatique - coordonne la planification de l'adaptation dans les différents ministères et agences. La collaboration 
interministérielle est définie dans le cadre de l'articulation des sept priorités stratégiques du Plan d'action national sur le changement climatique (NCCAP, 20112028). Ces priorités sont les suivantes: (i) la sécurité alimentaire; (ii) la suffisance en eau; (iii) la stabilité écologique et environnementale; (iv) la sécurité humaine; (v) les industries et services intelligents face au climat; (vi) l'énergie durable; et (vii) le renforcement des connaissances et des capacités.

La gestion de ces priorités transversales au sein du Ministère de l'agriculture implique un certain nombre de directions. Le Bureau of Soils and Water Management a été le plus efficace pour soutenir l'adoption de directives d'adaptation au niveau du terrain, grâce à son réseau de prestataires de services de vulgarisation. La Field Programs Operational Planning Division intègre les plans d'adaptation dans les opérations de routine du ministère, par exemple les évaluations avant et après une catastrophe. Le Systems-Wide Climate Change Office (SWCCO) a été chargé de coordonner ces multiples efforts, afin de faire face aux risques et aux vulnérabilités liés au changement climatique tout en mettant en œuvre le Plan national de modernisation de l'agriculture et de la pêche. La gestion de la foresterie fait partie du mandat du bureau de gestion des forêts du Ministère de l'environnement et des ressources naturelles. Le Philippine Master Plan for Climate Resilient Forestry Development (2016) a identifié l'adaptation comme l'un des axes de renforcement de la résilience et d'application de la loi sur le changement climatique de 2009 qui donne mandat à toutes les agences gouvernementales des Philippines d'intégrer l'adaptation au changement climatique dans tous les programmes et politiques. Les activités de planification de l'adaptation mettent fortement l'accent sur la gouvernance locale et à l'échelle du paysage. La loi philippine sur le changement climatique de 2009, inscrite dans la législation, stipule que les collectivités territoriales (LGUs) doivent formuler des plans locaux d'adaptation au changement climatique (LCCAPs) spécifiques. Le gouvernement a apporté son soutien en lançant le People's Survival Fund (Fonds de survie du peuple) avec une allocation annuelle d'un milliard de pesos philippins.

Afin de renforcer l'adoption de l'AbE articulée dans la CDN des Philippines, le programme PNA -Ag a soutenu l'élaboration des directives pour l'intégration des paysages dans les plans d'aménagement du territoire aux Philippines. Ces directives fournissent une justification de l'approche paysagère et suggèrent des actions pratiques pour leur utilisation afin d'intégrer l'adaptation et la RRC (Réduction des risques de catastrophe) dans les plans de développement agricole et les plans sectoriels des LGU, à l'intention des agents et des centres techniques agricoles régionaux et provinciaux. La foresterie joue un rôle important dans la mise en œuvre de la planification paysagère, par exemple dans les évaluations des risques et de la vulnérabilité ainsi que dans les évaluations de la capacité des terres. L'appui à la planification paysagère pour l'agriculture a été défini dans le cadre du processus du PNA, et une importance a été accordée au travail à la base, une consultation et une sensibilisation adéquates. Dans cette optique, la planification paysagère a été l'un des principaux sujets de dialogue politique menés aux niveaux national et infranational au cours de la phase de lancement du processus du PNA-Ag aux Philippines (élément A, étape 1). Les premiers efforts se sont concentrés sur le renforcement des capacités des agents de terrain nationaux et régionaux pour la mise en œuvre d'approches d'évaluation des risques climatiques et d'adaptation et de planification de la RCC basées sur le paysage. 



\section{Rapports, suivi et évaluation}

\author{
Ce chapitre \\ correspond à \\ l'élément $D$ des \\ orientations \\ techniques des PNA \\ qui mettent l'accent \\ sur la mise en place \\ de systèmes de suivi \\ et d'évaluation \\ efficaces.
}

\section{KUBU RAYA, KALIMANTAN} DE L'OUEST, INDONESIE

Les mangroves indonésiennes - Une jeune plante de mangrove pendant une marée haute durant l'étude conduit par le CIFOR sur la biomasse des mangroves en surface et en sous-sol, partie du Programme d'Adaptation et d'Atténuation Durables des Zones Humides (SWAMP).

๑CIFOR/Sigit Deni Sasmito
L'objectif ici est de s'assurer que le processus d'établissement de rapports, de suivi et d'évaluation établi pour le PNA est suffisamment axé sur le secteur forestier, ses priorités et ses besoins ainsi que sur les possibilités d'adaptation qu'il offre aux autres secteurs. Cette étape repose sur l'identification des domaines du processus du PNA qui méritent une attention particulière en termes de mesure des progrès, par exemple l'intégration du secteur dans le PNA, l'intégration de l'adaptation dans les stratégies et les plans en cours, ou la mise en œuvre. Elle comprend la définition d'indicateurs permettant d'évaluer les progrès du plan d'adaptation ainsi que les réalisations et les résultats découlant de la mise en œuvre du PNA . Cela exige que le secteur forestier soit intégré de manière appropriée dans le système d'établissement de rapports, de suivi et d'évaluation établi pour le PNA, que le secteur lui-même dispose d'outils et de moyens pour contribuer au système et à son amélioration. Il est particulièrement important de veiller à ce que le système mis en place ne fasse pas double emploi avec d'autres mécanismes d'établissement de rapport, mais qu'il s'appuie plutôt sur ces derniers.

\subsection{Préparer le suivi de la planification et de la mise en œuvre de l'adaptation}

Le suivi peut être effectué à plusieurs niveaux. Les projets et activités spécifiques décrits dans les PNA comprennent généralement des indicateurs permettant d'évaluer les produits et les résultats du projet. De manière générale, le processus des PNA doit intégrer des indicateurs pour le suivi de la mise en œuvre de l'ensemble du plan et l'évaluation des résultats des interventions d'adaptation. L'approche pour l'élaboration des indicateurs peut être double: dans un premier temps, des indicateurs à l'échelle de l'économie nationale peuvent être sélectionnés dans le PNA et conçus de manière à intégrer de façon appropriée les forêts et les secteurs arboricoles; ensuite, un nombre limité d'indicateurs spécifiques aux forêts, aux arbres et à l'agroforesterie peuvent être intégrés dans le PNA.

Ces indicateurs devraient faciliter le suivi des résultats, des effets et des impacts des options d'adaptation, tant sur les forêts que sur les populations. Ces indicateurs exigeraient un niveau suffisant de 
spécificité ou de granularité (par exemple sur le plan spatial), en particulier pour intégrer les points sensibles, par exemple pour le suivi des systèmes forestiers ou arboricoles d'importance capitale, soit du point de vue de la production et de l'économie, soit du point de vue de la protection de l'environnement ou des écosystèmes menacés (comme les mangroves). En outre, le suivi de la réussite de l'adaptation pour les personnes et les communautés nécessitera dans la plupart des cas la ventilation des indicateurs par sexe et par groupes sociaux, en accordant une attention particulière aux groupes identifiés comme les plus vulnérables.

La sélection des indicateurs s'appuiera autant que possible sur les données et les mécanismes existants.

À cet égard, elle peut s'appuyer, pour la foresterie, sur divers processus et outils existants tels que l'évaluation des ressources forestières mondiales (FRA) (voir encadré 14), les systèmes de suivi basés sur des données satellitaires tels que le GFW, les systèmes MRV avec REDD+ en cours d'élaboration ou disponibles dans de nombreux pays.
Les données recueillies dans le cadre de la FRA pourraient être particulièrement utiles car elles sont collectées au niveau national à intervalles réguliers et peuvent donc être utilisées pour évaluer les changements. Les ensembles de données suivants peuvent faire partie des données chiffrées collectés pour le suivi des PNA:

- l'étendue, les caractéristiques et l'évolution des forêts;

- le matériel forestier, la biomasse et le carbone;

- les objectifs de gestion des forêts, y compris les zones protégées et les plans de gestion à long terme;

- la protection des bassins versants;

- les perturbations forestières, y compris les incendies de forêt et les forêts dégradées;

- la superficie du domaine forestier permanent;

- NWFP;

- l'emploi.

\section{ENGADRÉ 14.}

\section{L'évaluation des ressources forestières mondiales (FRA)}

Depuis 1946, la FAO évalue l'état des ressources forestières mondiales à travers des rapports clés publiés tous les 5 à 10 ans. L'évaluation des ressources forestières mondiales (FRA) est désormais produite tous les cinq ans afin de fournir une approche cohérente de la description des forêts du monde et de leur évolution. L'évaluation est basée sur deux sources principales de données: Les rapports de pays élaborés par les correspondants nationaux et la télédétection qui est menée par la FAO en collaboration avec les points focaux nationaux et les partenaires régionaux. Pour l'évaluation des forêts de 2015, des données ont été compilées pour 234 pays et territoires: 155 rapports provenaient des pays eux-mêmes (couvrant 98,8 pour cent des forêts du monde) et les 79 autres étaient des études documentaires préparées par la FAO.

Le processus d'établissement de rapports par pays a été lancé en 2018 en vue de la prochaine FRA en 2020. L'option de fournir des mises à jour annuelles sur les variables clés a été introduite afin d'aider les pays à rendre compte de leurs progrès vers les ODD. Une nouvelle plateforme en ligne a été mise en place pour faciliter le processus de rapport et pour améliorer la transparence et la fiabilité des résultats, ainsi que le processus de révision, l'accessibilité et l'exploitation des données de la FRA pour les utilisateurs finaux. Les données résultantes ont été analysées au cours de l'année 2019 et les résultats finaux seront publiés en 2020 .

Pour plus d'informations sur l'évaluation des ressources forestières mondiales, voir: http://www.fao.org/forest-resources-assessment/en/ 
Le Forum des Nations unies sur les forêts (FNUF), soutenu par le Partenariat de collaboration sur les forêts (PCF), a proposé un ensemble commun d'indicateurs forestiers mondiaux (draft Global Core Set-GCS $)^{9}$ pour soutenir la mise en œuvre de l'Agenda 2030 et du plan stratégique sur les forêts. Le GCS comprend des indicateurs pour la gestion durable des forêts, des indicateurs pour les progrès vers les ODD et les objectifs liés aux forêts, et des indicateurs potentiels pour les objectifs du plan stratégique. Ces indicateurs qui mesurent la «santé» du secteur forestier peuvent également être considérés comme une source.

\subsection{Faire le suivi de la mise en œuvre et mettre à jour le PNA}

Le processus du PNA est un processus itératif. Cette étape du processus concerne le suivi et la mise à jour périodique du PNA, en tenant compte des nouvelles évaluations et connaissances ainsi que des changements de situation et également des résultats des activités d'adaptation mises en œuvre.

Il s'agit d'abord de revoir l'ensemble du projet de PNA afin de:

- vérifier que les priorités identifiées au point 7.1 sont correctement intégrées;

- présenter quelques contributions potentielles supplémentaires des forêts et des arbres à l'adaptation d'autres secteurs;

- introduire des mesures correctives si nécessaire.

Tout au long de la mise en œuvre du PNA, il est nécessaire d'évaluer la façon dont le plan lui-même est mis en œuvre, en utilisant les indicateurs définis au cours de l'étape décrite ci-dessus et les impacts qu'il a sur l'adaptation du secteur. Le Ministère de l'agriculture et des forêts chargé de l'adaptation en Finlande a mis au point une méthode d'évaluation de l'adaptation des secteurs par étapes (voir encadré 15). Cette méthode repose sur des questionnaires et des entretiens et souligne le fait que les activités intersectorielles, particulièrement importantes pour les forêts, les arbres et l'agroforesterie, se déroulent généralement à des stades ultérieurs du processus.

\section{ENGADRÉ 15.}

\section{Évaluation de l'adaptation des secteurs par étapes en Finlande}

En 2013, le Ministère finlandais de l'agriculture et des forêts a procédé à une évaluation du plan d'adaptation au changement climatique. Elle comprenait une évaluation du niveau d'adaptation par secteur en 2009 et 2013 à l'aide de l'évaluation réalisée en 2009 et du questionnaire d'évaluation ainsi que des entretiens réalisés en 2013. Elle identifie 5 niveaux d'adaptation.

\section{NIVEAU D'ADAPTATION}

\begin{tabular}{|l|l|}
\hline Étape 1 & besoin d'adaptation identifié par un groupe de pionniers du secteur; \\
& peu de recherches effectuées sur les impacts du changement climatique \\
& ou l'adaptation à celui-ci; \\
- quelques mesures d'adaptation identifiées mais non encore mises en œuvre. \\
\hline
\end{tabular}

9 Voir: Suivi, évaluation et rapport sur les progrès réalisés dans la mise en œuvre du plan stratégique des Nations unies pour les forêts 2017-2030, y compris l'instrument des Nations Unies sur les forêts et les contributions nationales volontaires. Agenda provisoire. http://www.cpfweb.org/487150dfc673f9958ed5b6909f2e5b45b711fc.pdf; http://www.cpfweb.org/96344/en/ 


\begin{tabular}{|l|l|}
\hline Étape 2 & $\begin{array}{l}\text { nécessité de mesures d'adaptation reconnues dans une certaine mesure } \\
\text { dans le secteur (certains décideurs); } \\
\text { impacts du changement climatique connus à titre indicatif (informations } \\
\text { qualitatives), avec une prise en compte de l'incertitude liée aux scénarios } \\
\text { de changement climatique; } \\
\text { - des mesures d'adaptation ont été identifiées et des plans ont été élaborés } \\
\text { pour leur mise en œuvre, dont certains ont été initiés. }\end{array}$ \\
\hline Étape 3 & $\begin{array}{l}\text { nécessité de mesures d'adaptation assez bien reconnue (majorité des } \\
\text { décideurs); }\end{array}$ \\
& $\begin{array}{l}\text { impacts du changement climatique assez bien connus (informations } \\
\text { quantitatives), avec une prise en compte de l'incertitude liée aux } \\
\text { scénarios de changement climatique; } \\
\text { mesures d'adaptation identifiées et mise en œuvre initiée; } \\
\text { - collaboration intersectorielle sur les mesures d'adaptation. }\end{array}$ \\
\hline Étape 4 & $\begin{array}{l}\text { nécessité de mesures d'adaptation largement reconnues et acceptées } \\
\text { dans le secteur; } \\
\text { - l'adaptation est intégrée dans les processus décisionnels réguliers; } \\
\text { impacts du changement climatique bien connus dans les limites de } \\
\text { l'incertitude liée aux scénarios de changement climatique; } \\
\text { - mise en œuvre de mesures d'adaptation largement initiée et évaluation } \\
\text { de leurs avantages, au moins dans une certaine mesure; la collaboration } \\
\text { intersectorielle sur les mesures d'adaptation est une pratique établie. }\end{array}$ \\
\hline Étape 5 & $\begin{array}{l}\text { mesures d'adaptation dans le cadre d'une stratégie d'adaptation, ou par } \\
\text { ailleurs reconnues et appliquées dans le secteur. }\end{array}$ \\
\hline
\end{tabular}

\subsection{Sensibilisation au processus et rapport sur les progrès et l'efficacité}

La diffusion de la documentation relative au processus du PNA, ainsi que les résultats du suivi, fait partie intégrante du processus. Elle est essentielle pour sensibiliser, assurer la transparence, garantir l'engagement et la coordination des acteurs et faciliter la mobilisation des ressources.

La sensibilisation doit être menée à différents niveaux, pour différents publics, avec une documentation et des canaux appropriés.
Plusieurs types de publics devraient faire l'objet d'une attention particulière: tout d'abord, les secteurs et les acteurs des forêts, des arbres et de l'agroforesterie au niveau national avec, le cas échéant, une communication ciblée pour des productions et des chaînes de valeur spécifiques; ensuite, une autre communication ciblée pourrait se concentrer sur les autres secteurs où il y a le plus d'interactions. En outre, une attention particulière devrait être accordée à la sensibilisation au niveau local/paysage, en impliquant autant que possible le large éventail d'acteurs qui sont impliqués dans l'utilisation des terres et la gestion des ressources naturelles, en particulier les cultures, l'élevage, la pêche et le secteur de l'eau. 


\section{Conclusion}

Les effets du changement climatique sur les forêts et les arbres sont déjà visibles partout dans le monde. Ils menacent non seulement les forêts elles-mêmes, mais aussi leur potentiel d'atténuation ainsi qu'un large éventail de services écosystémiques sur lesquels nous comptons. Ils sapent déjà la capacité des arbres et des forêts à soutenir l'adaptation des populations les plus vulnérables dans les zones rurales et les villes. Une action immédiate est nécessaire pour créer les conditions de l'adaptation des forêts et des populations qui en dépendent.

Le processus du PNA offre la possibilité de créer ces conditions. Puisqu'il s'agit d'un processus national qui englobe tous les secteurs de l'économie, il permet de prendre en considération toutes les interactions entre les forêts, les arbres et tous les autres secteurs connexes: agriculture, eau, utilisation des terres, infrastructures, énergie, biodiversité..., d'identifier les principales conditions requises pour renforcer les contributions des forêts à la réduction des risques climatiques et à l'adaptation à ces risques, et de mettre en place l'environnement favorable nécessaire. L'efficacité du processus dépendra de sa capacité à intégrer tous les engagements, stratégies et plans pertinents, à impliquer toutes les organisations et tous les acteurs concernés, en particulier les plus vulnérables, et à inclure différents niveaux de prise de décision, des chaînes de valeur aux paysages, afin de prendre en compte de manière appropriée les vulnérabilités et les agences spécifiques.

Lorsqu'on lui accorde l'importance qu'il mérite au niveau national, le processus du PNA peut être un vecteur de mise en œuvre majeur, non seulement pour les CDN mais également pour les ODD, en considérant, notamment en ce qui concerne les forêts et les arbres, les liens qui existent entre les objectifs d'atténuation, d'adaptation et de développement durable. En tant que tel, il peut encadrer la planification des actions et, en fin de compte, des projets individuels sur le terrain. D'un autre côté, puisqu' il s'agit d'un processus itératif, il permettra en retour de fournir des renseignements pour l'évaluation périodique des $C D N$. 


\section{Annexes}

Annexe 1: Glossaire

Annexe 2: Liste de contrôle des éléments de l'inventaire

Annexe 3: Outils de connaissance

Annexe 4: Sous-étapes et méthodologies pour prioriser les options d'adaptation 


\section{Annexe 1: Glossaire}

Adaptation: ajustement des systèmes naturels ou humains en réponse à des stimuli climatiques réels ou attendus ou à leurs effets, qui atténue les dommages ou exploite les opportunités de bénéfices. On peut distinguer différents types d'adaptation, notamment l'adaptation anticipée, autonome et planifiée (GIEC, 2007).

Adaptation basée sur les écosystèmes: Une approche de l'adaptation qui intègre l'utilisation de la biodiversité et des services écosystémiques dans une stratégie globale pour aider les gens à s'adapter aux effets néfastes du changement climatique. Elle comprend la gestion durable, la conservation et la restauration des écosystèmes afin de fournir des services qui aident les gens à s'adapter à la fois à la variabilité actuelle du climat et au changement climatique. L'adaptation fondée sur les écosystèmes contribue à réduire la vulnérabilité et à accroître la résilience aux risques climatiques et non climatiques et procure de multiples avantages à la société et à l'environnement (Colls, Ash et Ikkala, 2009).

Agriculture intelligente face au climat: Une approche qui contribue à orienter les actions nécessaires pour transformer et réorienter les systèmes agricoles afin de soutenir efficacement le développement et d'assurer la sécurité alimentaire dans un climat en mutation. L'AIC vise à atteindre trois objectifs principaux: accroître durablement la productivité et les revenus agricoles; s'adapter et renforcer la résilience au changement climatique; et réduire et/ou supprimer les émissions de gaz à effet de serre (GES), dans la mesure du possible (FAO, 2014b).

Agroforesterie: nom collectif pour les systèmes et technologies d'utilisation des terres où des végétaux ligneux pérennes (arbres, arbustes, palmiers, bambous, etc.) sont délibérément utilisées dans les mêmes unités de gestion des terres que les cultures agricoles et/ou les animaux, dans une certaine forme d'arrangement spatial ou de séquence temporelle (Lundgren et Raintree, 1982).

Approchepaysagère:Uneapprochedu développement durable qui traite des processus à grande échelle de manière intégrée et multidisciplinaire, en combinant la gestion des ressources naturelles avec des considérations environnementales et de moyens d'existence. Elle diffère des approches écosystémiques par le fait qu'elle peut inclure de multiples écosystèmes. L'approche paysagère tient également compte des activités humaines et de leurs institutions, en les considérant comme faisant partie intégrante du système plutôt que comme des agents externes (FAO, 2012).
Avantages de l'adaptation: le coût des dommages évités ou les bénéfices cumulés découlant de l'adoption et de la mise en œuvre de mesures d'adaptation (GIEC, 2007).

Capacité d'adaptation: Capacité d'un système de s'adapter aux changements climatiques (notamment à la variabilité du climat et aux phénomènes extrêmes), afin d'en atténuer les dommages potentiels, de tirer parti des opportunités offertes ou de faire face aux conséquences. (GIEC, 2007).

Changement climatique: toute modification du climat au fil du temps, qu'elle soit due à la variabilité naturelle ou à l'activité humaine (GIEC, 2007).

Changement transformationnel: changement profond, systémique et durable pouvant avoir un impact à grande échelle

Coûts d'adaptation: Coûts de planification, de préparation, de facilitation et de mise en œuvre des mesures d'adaptation, y compris les coûts de transition (GIEC, 2007).

Évaluation de l'impact du changement climatique: Pratique consistant à identifier et à évaluer, en termes monétaires et/ou non monétaires, les effets du changement climatique sur les systèmes naturels et humains. Les impacts potentiels sont tous les impacts qui peuvent se produire en raison d'un changement climatique prévu, sans tenir compte de l'adaptation. Les impacts résiduels sont les impacts du changement climatique qui se produiraient après l'adaptation (FAO, 2014b).

Exposition aux événements liés au changement climatique: «La nature et le degré d'exposition d'un système à des variations climatiques importantes». (GIEC, 2001)

Foresterie urbaine: Pratique consistant à gérer les forêts urbaines afin d'assurer leur contribution optimale au bien-être physiologique, sociologique et économique des sociétés urbaines. Forêt urbaine d'une ville: réseaux ou systèmes comprenant toutes les zones boisées, les groupes d'arbres et les arbres individuels situés dans les zones urbaines et périurbaines; ils comprennent donc les forêts, les arbres des rues, les arbres des parcs et jardins et les arbres des coins abandonnés.

Gestion des risques de catastrophes: Processus de recours systématique aux directives, compétences opérationnelles, capacités et organisations administratives pour la mise en œuvre de politiques, stratégies et capacités d'adaptation améliorées afin de réduire les effets négatifs des aléas et les risques de catastrophe (UNISDR, 2009). 
Impact du changement climatique: les effets du changement climatique sur un système humain ou naturel exposé et ses composantes. Cela comprend les vies, les moyens d'existence, la santé, les dimensions économiques, sociales et culturelles, les services disponibles, les infrastructures, les écosystèmes et l'environnement (FAO, 2014b).

Institutions: organisations et contrats formels, ainsi que normes et conventions sociales et culturelles informelles, qui opèrent au sein et entre les organisations et les individus (FAO, 2014b).

Intégration de l'adaptation: l'intégration des objectifs, stratégies, politiques, mesures ou opérations d'adaptation de telle sorte qu'ils fassent partie des politiques, processus et budgets de développement national et régional à tous les niveaux et étapes (Lim and Spanger-Siegfred, eds., 2005).

Maladaptation: tout changement dans les systèmes naturels ou humains conduisant à augmenter par inadvertance la vulnérabilité aux stimuli climatiques; une adaptation qui ne parvient pas à réduire la vulnérabilité mais qui au contraire, l'augmente (GIEC, 2001).

Pénurie d'eau: le stade à partir duquel le cumul des impacts de tous les utilisateurs a un effet préjudiciable sur l'approvisionnement en eau ou la qualité de l'eau dans le cadre des dispositions institutionnelles en vigueur, au point que la demande de tous les secteurs, dont l'environnement, ne peut être entièrement satisfaite (ONU-Eau, 2014).

Réduction des risques de catastrophes: concept et pratique de réduction des risques de catastrophes par des efforts systématiques d'analyse et de gestion des facteurs de causalité des catastrophes, notamment par la réduction de l'exposition aux dangers, la diminution de la vulnérabilité des personnes et des biens, la gestion judicieuse des terres et de l'environnement, l'amélioration de la préparation aux événements indésirables. (UNISDR, 2009).

Résilience: capacité d'un système social ou écologique à absorber des perturbations tout en conservant la même structure de base et les mêmes modes de fonctionnement, la capacité d'autoorganisation et la capacité d'adaptation au stress et au changement (GIEC, 2007).

Risques: processus, phénomène ou activité humaine pouvant entraîner des pertes de vie, des blessures ou d'autres effets sur la santé, des dommages matériels, des perturbations sociales et économiques ou une dégradation de l'environnement (AGNU. 2016. Rapport du groupe de travail intergouvernemental d'experts à composition non limitée chargé des indicateurs et de la terminologie relatifs à la réduction des risques de catastrophe. (1er décembre 2016).

Risque naturel: processus ou phénomène naturel pouvant entraîner des pertes de vies humaines, des blessures ou d'autres effets sur la santé, des dommages matériels, la perte de moyens d'existence et de services, des perturbations sociales et économiques ou des dommages environnementaux (FAO, 2014b).

Secteurs de l'agriculture: pour la FAO, l'agriculture couvre les systèmes de culture et d'élevage, y compris les parcours et les pâturages, les forêts, la pêche et l'aquaculture et les ressources connexes qu'ils utilisent (eau, terre, sols, ressources génétiques et biodiversité). Lorsque la discussion concerne un secteur agricole spécifique, ceci est précisé dans le text.

Sensibilité à la variabilité ou au changement climatique: degré auquel un système est affecté, soit de manière négative ou bénéfique, par la variabilité ou le changement climatique. L'effet peut être direct (par exemple, une modification du rendement des cultures en réponse à un changement de la moyenne, de la gamme ou de la variabilité des températures) ou indirect (par exemple, les dommages causés par une augmentation de la fréquence des inondations côtières due à l'élévation du niveau de la mer) (FAO, 2014b).

Variabilité du climat: variations du climat (mesurées par comparaison avec l'état moyen et d'autres statistiques telles que les écarts types et les statistiques des valeurs extrêmes) à toutes les échelles temporelles et spatiales au-delà de celles des événements météorologiques individuels. La variabilité peut être due à des processus internes naturels au sein du système climatique (variabilité interne) ou à des variations de la pression externe naturelle ou anthropique (variabilité externe) (GIEC, 2007).

Vulnérabilité: la propensión o predisposición a verse adversamente afectados; en función de los posibles impactos (exposición y sensibilidad a la exposición) y la capacidad adaptativa (FAO, 2014b).

ÉTAT DE MINAS GUEIRAS, BRÉSIL

Les producteurs locaux ont développé un système agricole qui combine la cueillette de fleurs, l'agroforesterie, le pâturage du bétail et la culture des champs. CFAO/Valda Nogueira / FAO 


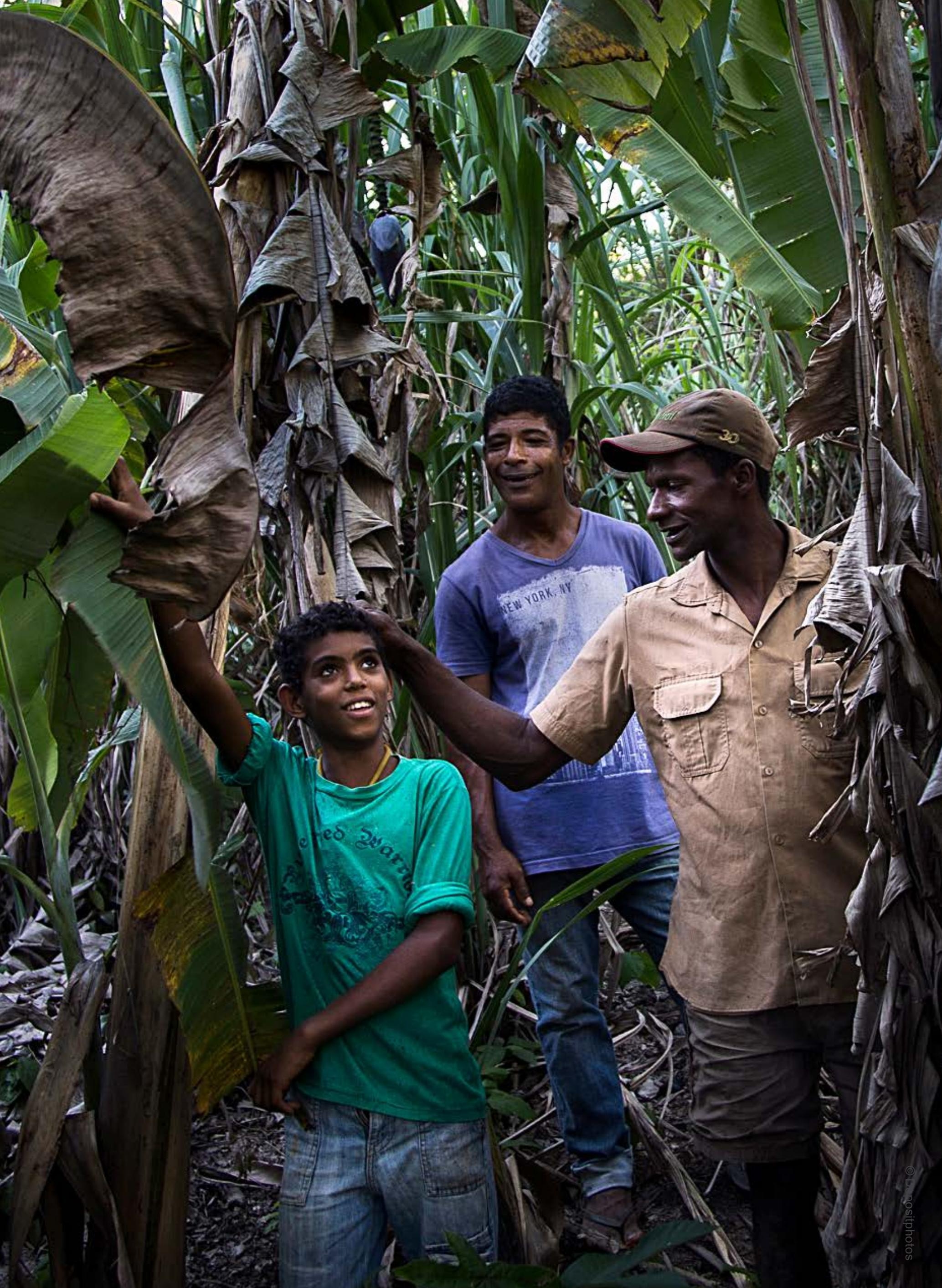




\section{Annexe 2. Liste de contrôle des éléments de l'inventaire}

Cette liste de contrôle vise à faciliter l'inventaire (voir section 5.1). Elle a été préparée dans le cadre du programme PNA -Ag et adaptée à la foresterie et à l'agroforesterie, en respectant sa structure globale pour faciliter l'intégration de la foresterie aux autres sous-secteurs de l'agriculture (cultures, élevage, pêche et aquaculture).

\begin{tabular}{|c|c|c|c|c|}
\hline APERÇU & & $\begin{array}{l}\text { SECTION INFORMATIONS } \\
\text { SUR LES PAYS }\end{array}$ & & \\
\hline $\begin{array}{l}\text { INVENTAIRE DU } \\
\text { PNA -AG }\end{array}$ & DESCRIPTION & $\begin{array}{l}\text { INFORMATIONS } \\
\text { DISPONIBLES - [pays] }\end{array}$ & SOURCE & ANNÉE \\
\hline \multicolumn{5}{|l|}{ Table des matières } \\
\hline \multicolumn{5}{|c|}{ Liste des chiffres et tableaux } \\
\hline \multicolumn{5}{|c|}{ Acronymes et abréviations } \\
\hline \multicolumn{5}{|c|}{ Résumé } \\
\hline \multicolumn{5}{|l|}{ 1. Introduction } \\
\hline & $\begin{array}{l}\text { Si elle est publiée sous forme de rapport: } \\
\text { Donner les raisons de la réalisation d'un } \\
\text { inventaire, par exemple demande d'appui, } \\
\text { programmes d'appui en vue du PNA ou du PNA } \\
\text {-Ag, objectifs de l'inventaire. }\end{array}$ & & & \\
\hline & $\begin{array}{l}\text { Présentation du processus des PNA et du } \\
\text { processus d'intégration de la foresterie et de } \\
\text { l'agroforesterie dans les PNA. }\end{array}$ & & & \\
\hline \multicolumn{5}{|c|}{$\begin{array}{l}\text { 2. Analyse du contexte, des processus et des acteurs concernés par } \\
\text { le PNA dans le [pays]. }\end{array}$} \\
\hline $\begin{array}{l}\text { a. Présentation de } \\
\text { la foresterie, de } \\
\text { l'agroforesterie } \\
\text { et des secteurs } \\
\text { connexes dans le } \\
\text { [pays] }\end{array}$ & $\begin{array}{l}\text { Aperçu de la foresterie et de l'agroforeste- } \\
\text { rie, y compris la proportion de la population } \\
\text { concernée, les principales régions agroéco- } \\
\text { logiques, les types de systèmes forestiers et } \\
\text { agroforestiers, les taux de croissance et de } \\
\text { production, la contribution économique et les } \\
\text { profils d'exportation, l'emploi, la lutte contre } \\
\text { les maladies. }\end{array}$ & & & \\
\hline $\begin{array}{l}\text { b. Vulnérabilité de } \\
\text { la foresterie et de } \\
\text { l'agroforesterie } \\
\text { au changement } \\
\text { climatique }\end{array}$ & $\begin{array}{l}\text { Résumé des principaux impacts, risques, y } \\
\text { compris les projections utilisées. Défis exis- } \\
\text { tants en matière de gestion de l'environne- } \\
\text { ment et des ressources naturelles, exacerbés } \\
\text { par le changement climatique, par exemple } \\
\text { en ce qui concerne l'hydrologie et la qualité } \\
\text { des sols. }\end{array}$ & & & \\
\hline \multicolumn{5}{|l|}{$\begin{array}{l}\text { c. Politiques, lois } \\
\text { et autres points } \\
\text { d'entrée pour } \\
\text { l'adaptation au } \\
\text { changement } \\
\text { climatique dans } \\
\text { le domaine de la } \\
\text { foresterie et de } \\
\text { l'agroforesterie }\end{array}$} \\
\hline $\begin{array}{l}\text { - Cadres de } \\
\text { planification du } \\
\text { développement } \\
\text { national et } \\
\text { sectoriel }\end{array}$ & $\begin{array}{l}\text { L'adaptation au changement climatique pour } \\
\text { la foresterie et l'agroforesterie dans les plans } \\
\text { de développement nationaux. }\end{array}$ & $\begin{array}{l}\text { Par exemple, le cadre } \\
\text { d'adaptation au chan- } \\
\text { gement climatique } \\
\text { (2015-2020) contient } \\
\text { une politique/un mé- } \\
\text { canisme de coordina- } \\
\text { tion de l'adaptation de } \\
\text { la foresterie dans le } \\
\text { pays x }\end{array}$ & $\begin{array}{l}\text { Par exemple, } \\
\text { la stratégie } \\
\text { nationale } \\
\text { sur le } \\
\text { changement } \\
\text { climatique, } \\
2015\end{array}$ & \\
\hline
\end{tabular}




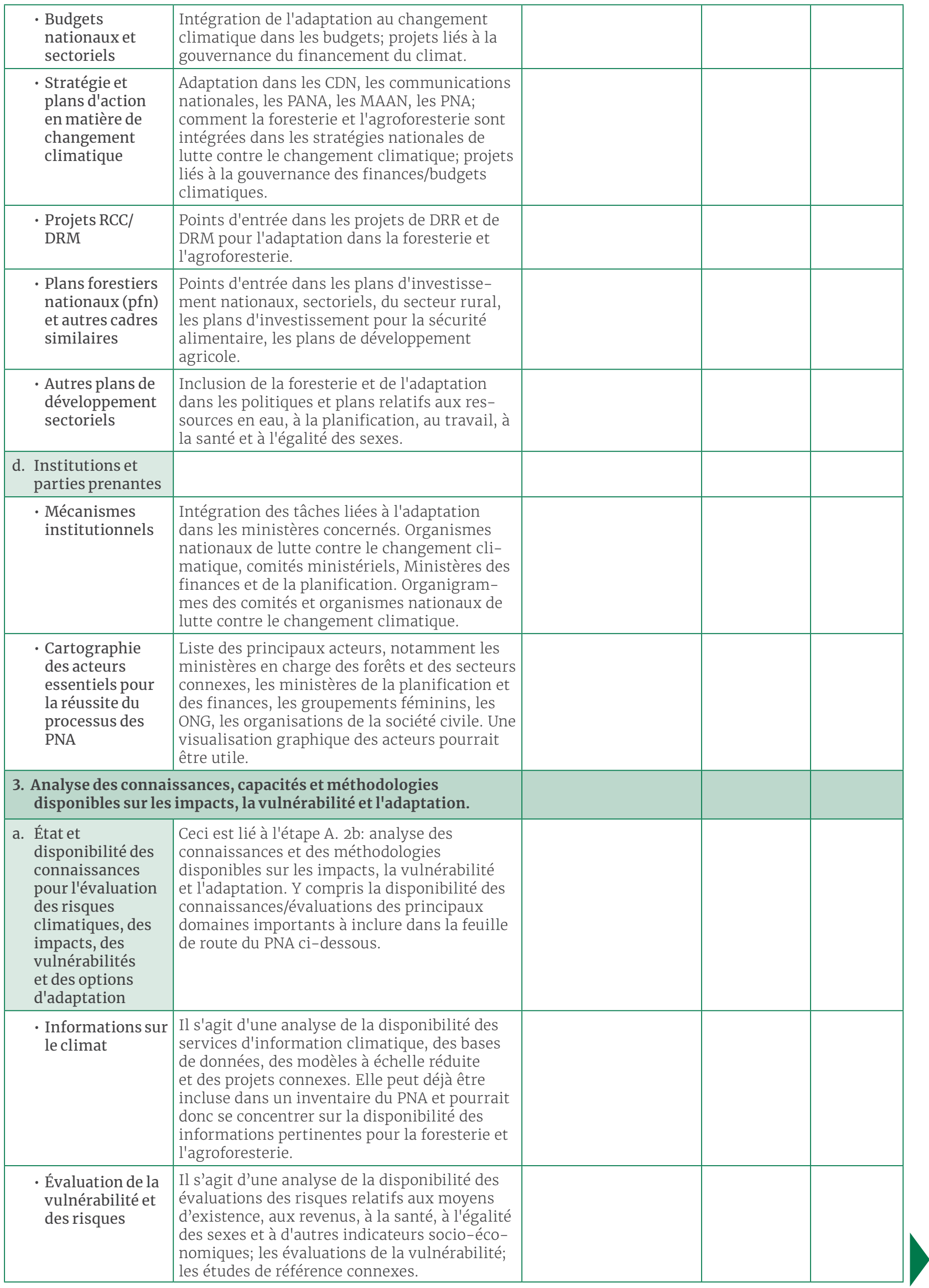




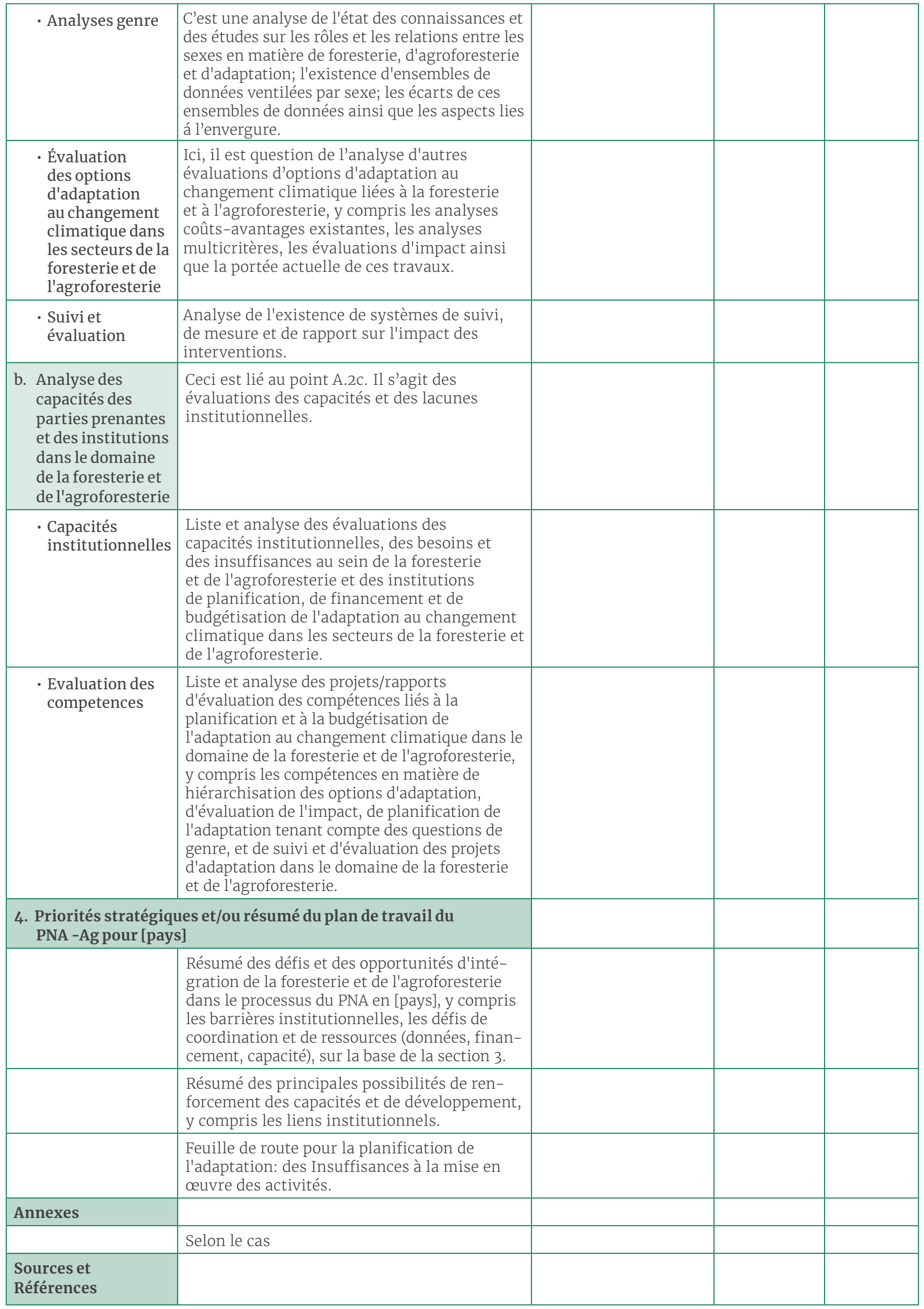




\section{Annexe 3: Outils de connaissance}

Vous trouverez ci-dessous à titre indicatif une liste non exhaustive d'outils de connaissance qui peuvent être utiles à différents stades du processus du PNA . Elle est organisée autour de thèmes centraux liés à la collecte de données et aux impacts du changement climatique sur les forêts (1-2), aux vulnérabilités et aux risques (3-6), aux écosystèmes spécifiques (mangroves 7) ou à des groupes (8-9), aux services des écosystèmes et à la sécurité alimentaire (10-13), à la gestion des forêts et à l'adaptation (14-16).

1) Collecte de données et modélisation

2) Changement climatique et forêts

3) Évaluations de la vulnérabilité

4) Événements météorologiques extrêmes

5) Ravageurs et maladies

6) Feux de brousse

7) Mangroves

8) Les peuples indigènes

9) Genre

10) Services écosystémiques

11) Eau

12) Biodiversité

13) Sécurité alimentaire et nutrition

14) Gestion des forêts

15) Adaptation

16) Restauration des forêts et des paysages

\section{1) LA COLLECTE DE DONNÉES ET LA MODÉEISATIONN}

Zonage agroécologique mondial (GAEZ): fournit une évaluation, historique et future, des ressources et du potentiel agricoles, y compris les ressources en terre et en eau, les ressources agroclimatiques, l'adéquation et les rendements potentiels, les rendements réels et la production à échelle réduite et les écarts de rendement et de production. http://www.fao.org/nr/gaez/en/\#

Systèmedemodélisationdesimpactsduchangement climatique sur l'agriculture a (MOSAICC): Méthodologie et système de modèles conçus pour réaliser une évaluation interdisciplinaire des impacts du changement climatique sur l'agriculture par le biais de simulations. Les principales composantes du système sont un portail de réduction d'échelle statistique permettant de réduire les données des Modèles de Circulation Générale (MCG) aux réseaux de stations météorologiques, un modèle hydrologique pour l'estimation des ressources en eau pour l'irrigation dans les principaux bassins, deux modèles de cultures basés sur le bilan hydrique pour simuler les rendements des cultures dans le cadre de scénarios de changement climatique et un modèle pour évaluer l'effet de l'évolution des rendements sur les économies nationales. http://www.fao.org/in-action/mosaicc/en/

Agriculture Stress Index Systems (ASIS). Au niveau mondial et national: En utilisant des données sur la végétation et la température de la surface des terres, l'ASIS surveille les indices de végétation et détecte les points chauds où les cultures peuvent être affectées par la sécheresse. http://www.fao.org/ resilience/news-events/detail/en/c/296089/

Open Foris (y compris le Système d'accès, de traitement et d'analyse des données d'observation de la Terre (SEPAL), Collect Earth, Collect Earth Online et Earth Map): est un ensemble d'outils logiciels à source ouverte de la FAO pour faciliter la collecte, l'analyse et la communication de données de manière souple et efficace. Ses plateformes, outils et modules peuvent être utilisés pour les inventaires forestiers, l'évaluation de l'utilisation des terres et des changements d'affectation des terres, et les rapports sur le changement climatique. wWW.openforis.org

\section{2) LECHANGEMENT CLIMATIQUE ET LES FORÊTS}

Le portail de données s CCAFS-Climat. Il fournit des ensembles de données climatiques mondiales et régionales à haute résolution qui servent de base pour évaluer les impacts du changement climatique et l'adaptation à celui-ci dans divers domaines, notamment la biodiversité, l'agriculture, l'agroclimatologie, les services écosystémiques et l'hydrologie. http://ccafs-climate.org/

Agroforesterie et changement climatique: Enjeux, défis et perspectives (2019). Ce volume est une vraie mine d'informations précieuses sur les nouvelles technologies écologiques et leur rôle potentiel dans la lutte contre le changement climatique via l'agroforesterie. https://www.taylorfrancis.com/ books/e/9780429057274 
Perturbations forestières dans le cadre du changement climatique (2017) Il s'agit d'un article de synthèse sur les perturbations forestières qui sont sensibles au climat. La compréhension de la dynamique des perturbations en réponse aux changements climatiques est incomplète, notamment en ce qui concerne les modèles à grande échelle, les effets d'interaction et les rétroactions atténuantes. Cet article présente une synthèse globale des effets du changement climatique sur les principaux facteurs de perturbation abiotiques (feu, sécheresse, vent, neige et glace) et biotiques (insectes et agents pathogènes). https://www.nature.com/articles/nclimate3303

Le changement climatique et les forêts européennes: que savons-nous, quelles sont les incertitudes et quelles sont les implications pour la gestion des forêts? (2014). L'évolution récente des observations et des projections en matière de changement climatique, les impacts observés et prévus sur les forêts européennes et les incertitudes associées sont examinés et synthétisés afin de comprendre les implications pour la gestion des forêts.

https://www.ncbi.nlm.nih.gov/pubmed/25156267

Lignes directrices sur le changement climatique à l'intention des gestionnaires (2013).Ces directives sont publiées par la FAO pour aider les gestionnaires de forêts à répondre aux défis et aux opportunités du changement climatique au niveau des unités de gestion forestière. http://www.fao.org/3/i3383e/i3383e.pdf

Boîte à outils du CIFOR sur les forêts et le changement climatique (2010). Cette boîte à outils a été développée par le Centre pour la recherche forestière internationale afin de renforcer la compréhension et les compétences techniques sur les questions du changement climatique et des forêts, y compris l'atténuation, l'adaptation, le bilan carbone et les marchés du carbone, et les biocarburants. La boîte à outils se compose d'une série de présentations PowerPoint avec des notes d'accompagnement. www.cifor.cgiar.org/fctoolbox/

Impacts de la structure et de la gestion des paysages forestiers sur la production de bois et les stocks de carbone dans l'écosystème de la forêt boréale dans le cadre du changement climatique (2007). Dans ce document, un modèle de croissance et de rendement basé sur les processus a été utilisé pour étudier la sensibilité de la production de bois et des stocks de carbone à la gestion dans le cadre de différents scénarios climatiques au niveau de l'UGF. Les effets des distributions initiales des classes d'âge d'une UGF sur la production de bois et les stocks de carbone dans différents scénarios de gestion et de climat ont également été examinés et les implications sur le coût de la séquestration du carbone au cours des 100 prochaines années ont été présentées. https://www.sciencedirect.com/science/ article/pii/S0378112707000412.

\section{3) LES ÉVALUATIONS DE LA VULNÉRABILITÉ}

Évaluation de la vulnérabilité des forêts et des populations dépendantes des forêts au changement climatique: une méthodologie cadre (2019). Cette publication de la FAO et de la FTA fournit des conseils techniques pratiques pour l'évaluation de la vulnérabilité des forêts dans le contexte du changement climatique. Elle décrit les éléments qui doivent être pris en compte pour différents horizons temporels et présente une approche structurée pour la réalisation de ces évaluations. Le cadre guidera les praticiens dans la conduite d'une analyse étape par étape et facilitera le choix et l'utilisation d'outils et de méthodes appropriés. https://doi.org/10.4060/CA7064EN

Mesure et analyse de l'indice de résilience (RIMA-II): une approche quantitative innovante qui permet d'expliquer pourquoi et comment certains ménages font face aux chocs et aux facteurs de stress mieux que d'autres. La mesure directe fournit des informations descriptives sur la capacité de résilience des ménages et constitue un outil d'analyse politique précieux pour informer les gouvernements, les organisations internationales, les donateurs et la société civile sur les décisions de financement et de politique générale, car elle permet de cibler et de classer les ménages, du plus résistant au moins résistant. RIMA-II mesure également la résilience de manière indirecte afin de fournir des preuves sur les principaux déterminants de la capacité de résilience des ménages. La mesure indirecte de la résilience peut être adoptée comme un outil de prévision pour les interventions qui renforcent la résilience à l'insécurité alimentaire. http://www.fao.org/3/i5665e/i5665e.pdf

Un examen des approches et des méthodes existantes pour évaluer la vulnérabilité des forêts et des populations tributaires de la forêt au changement climatique (2018). Une publication de la FAO sur les approches d'évaluation de la 
vulnérabilité. Celles-ci peuvent être classées en fonction de l'objectif qu'elles visent. La vulnérabilité contextuelle aborde les problèmes actuels du climat et est généralement évaluée à l'aide de techniques participatives avec les personnes qui vivent ou travaillent dans les forêts. La vulnérabilité des résultats examine la vulnérabilité biophysique des forêt ; elle est souvent utilisée pour évaluer les causes et les effets du changement climatique sur un système biologique. Les évaluations de la vulnérabilité peuvent être très techniques et quantitatives, avec l'utilisation de programmes informatiques et de systèmes d'informations géographiques de pointe. Elles peuvent également être basées sur des approches de sciences sociales pour obtenir des informations qualitatives provenant des populations. http://www.fao.org/3/CA2635EN/ca2635en.pdf

Une analyse de risque multicritères pour évaluer les impacts des alternatives de gestion forestière sur la santé des forêts en Europe (2012). Une évaluation de l'effet de quatre alternatives de gestion forestière (FMA) (c'est-à-dire une gestion proche de la nature, une gestion extensive avec des objectifs combinés, des plantations intensives équiennes et une foresterie à courte rotation pour la production de biomasse) sur les risques de dommages biotiques et abiotiques dans huit études de cas régionales. https://www.ecologyandsociety.org/vol17/iss4/art52/

Adapter lagestion durable des forêts au changement climatique: scénarios pour l'évaluation de la vulnérabilité (2012). Ce rapport traite des origines des scénarios qui seront nécessaires pour évaluer les impacts du changement climatique et d'autres facteurs de stress sur les systèmes forestiers gérés. Il examine comment les scénarios peuvent être construits pour être appliqués à l'échelle locale (comme une unité de gestion forestière), en utilisant à la fois des approches descendantes (réduction d'échelle à partir de projections mondiales et régionales) et ascendantes (prise en compte des tendances et des projections locales). Des exemples pratiques d'utilisation de scénarios pour l'évaluation de l'impact dans le domaine de la foresterie sont brièvement passés en revue dans quatre études de cas provenant de tout le Canada. https://www. cabdirect.org/cabdirect/abstract/20133150126

Une évaluation de la vulnérabilité des forêts australiennes aux impacts du changement (2011). Ce projet fait partie de la composante « Synthèse et recherche intégrative » des activités du NCCARF. Il aborde le manque de connaissances en ce qui concerne notre compréhension des implications du changement climatique pour le domaine forestier australien, y compris les forêts indigènes, les plantations, la foresterie agricole et les plantations environnementales. Il vise à améliorer la compréhension des connaissances actuelles sur les conséquences biophysiques et socio-économiques probables du changement climatique pour les régions forestières indigènes et plantées d'Australie. Il évalue la vulnérabilité des forêts australiennes tant du point de vue de l'utilisation des ressources que des services écosystémiques, en identifiant les forêts et les communautés particulièrement vulnérables dans les principales zones forestières. Il fournit également des informations sur ce qui est fait en Australie pour comprendre et gérer les risques liés au climat en relation avec les forêts, et il offre des conseils sur les principales lacunes pour aider à l'adaptation au changement climatique. https://www.nccarf.edu.au/content/assessmentvulnerability-australian-forests-impactsclimate-change

Un système d'évaluation de la vulnérabilité des espèces (SAVS) au changement climatique (2011). Le SAVS est un outil simple et flexible conçu pour les gestionnaires afin d'évaluer le risque relatif des espèces individuelles aux déclins de population en réponse aux changements climatiques et aux phénomènes connexes prévus. Le SAVS utilise un questionnaire facile à remplir, basé sur des critères prédictifs qui traduisent les réponses pour les espèces vertébrées terrestres en scores indiquant la vulnérabilité ou la résilience au changement climatique. Le SAVS comprend également des méthodes de calcul de l'incertitude, des instructions détaillées pour noter une espèce et des directives pour l'utilisation de l'outil. www.fs.fed.us/rm/pubs/rmrs_gtr257.pdf

Le changement climatique dans un paysage vivant: aspects conceptuels et méthodologiques d'une évaluation de la vulnérabilité dans la Cordillera Real Est de la Colombie, de l'Équateur et du Pérou (2011).Ce document du Fonds mondial pour la nature présente les résultats d'analyses de vulnérabilité combinées (biologique, hydrologique et socio-économique). Les résultats démontrent la nécessité de prendre des mesures visant à maintenir la fourniture continue de services écosystémiques ainsi que les richesses biologiques et culturelles de la région. Les mesures d'adaptation prioritaires comprennent des actions visant à développer et à renforcer les capacités et les systèmes de 
production des communautés et institutions locales, dans le but de maintenir et de rétablir la résilience des écosystèmes, de renforcer un cadre politique régional avec des considérations de vulnérabilité et d'adaptation au changement climatique, et de renforcer la capacité à générer et à diffuser les informations nécessaires pour accroître la participation des citoyens aux processus décisionnels. http://awsassets.panda.org/ downloads/cc_ecr_final_web.pdf

Méthodes et outils pour évaluer la vulnérabilité des forêts et des populations au changement climatique (2009). Ce document de travail du Centre pour la recherche forestière internationale donne un aperçu des méthodes et des outils appropriés pour évaluer la vulnérabilité des forêts, des services des écosystèmes forestiers et des personnes ou secteurs dépendant de la forêt au changement climatique. Il fournit une typologie des méthodes et des outils et donne des exemples. www.cifor.cgiar.org/ publications/pdf_files/WPapers/WP43Locatelli.pdf

CRiSTAL (2009). Le Community-based Risk Screening Tool - Adaptation and Livelihoods (CRiSTAL) est conçu pour aider les planificateurs et les gestionnaires de projets à intégrer l'adaptation au changement climatique et la réduction des risques dans les projets communautaires. www.iisd.org/cristaltool/

Un cadre pour l'évaluation de la vulnérabilité des communautés forestières au changement climatique (2007).(Cette publication présente un cadre général et une approche pour évaluer la vulnérabilité des communautés forestières au changement climatique et les risques potentiellement accrus associés à ce changement. Elle identifie les éléments spécifiques qui devraient être pris en compte dans l'évaluation de la vulnérabilité et décrit une série d'étapes que les chercheurs et les communautés peuvent suivre pour déterminer systématiquement les sources de vulnérabilité au changement climatique. https://cfs.nrcan.gc.ca/pubwarehouse/pdfs/27507.pdf

Compendium sur les méthodes et outils d'évaluation des impacts du changement climatique, de la vulnérabilité et de l'adaptation à ce phénomène (2005). Ce compendium fournit une synthèse des informations clés sur les cadres et outils disponibles pour évaluer la vulnérabilité au changement climatique et les options d'adaptation, les caractéristiques particulières de chaque cadre ou outil, et des informations sur la manière d'obtenir de la documentation, des formations ou des publications qui se rapportent á chaque outil. https://unfccc.int/files/adaptation/methodologies for/vulnerability_and_adaptation/application/ pdf/200502_compendium_methods_tools_2005.pdf

Évaluer la vulnérabilité des forêts urbaines australiennes aux extrêmes climatiques (2019). Les forêts urbaines sont reconnues pour les multiples avantages qu'elles procurent aux citadins. Toutefois, le changement climatique aura des répercussions sur la survie et la persistance des espèces d'arbres dans les écosystèmes urbains. Les défaillances des arbres entraîneront des pertes économiques et compromettront la fourniture de bénéfices sociétaux. Les effets du changement climatique dépendront de la résilience et de la capacité d'adaptation des espèces, ainsi que des mesures de gestion susceptibles d'atténuer certains des effets négatifs. Ici, nous avons évalué la vulnérabilité potentielle des forêts urbaines australiennes aux extrêmes climatiques. https://nph.onlinelibrary.wiley.com/doi/full/10. 1002/ppp3.10064

\section{4) LES ÉVÉNEMENTS METEOROLOGIQUES EXTRÊMES}

Impacts du changement climatique, extrêmes climatiques et stratégies alternatives dans les forêts gérées (2019.)Le taux de croissance de la plupart des espèces d'arbres dans les forêts boréales augmentera avec le changement climatique. Cette hausse est contrebalancée par un risque accru de dommages dus à des événements météorologiques extrêmes. On pense que le risque de dommages causés par les tempêtes augmentera avec le temps, surtout si les forêts continuent d'être gérées comme elles le sont aujourd'hui. Dans cette étude, un nouveau modèle hybride de croissance forestière à l'échelle du paysage 3PG-Heureka a été développé et des simulations ont été réalisées pour prédire les dommages causés par les tempêtes dans le comté de Kronoberg, sur une période de 91 ans (2010-2100) avec différents régimes de gestion alternatifs selon divers scénarios climatiques (historique, RCP4.5 et RCP8.5). Les résultats indiquent que les dommages causés par les tempêtes pourraient réduire considérablement l'augmentation annuelle du volume et les revenus nets annuels obtenus des paysages forestiers si les régimes de gestion forestière actuels sont utilisés. https://www.tandfonline.com/doi/full/10.1080/1195 6860.2018.1515597 
La santé des forêts et le changement planétaire (2015). Bien que les forêts indigènes soient adaptées à un certain niveau de perturbation, toutes les forêts sont aujourd'hui confrontées à de nouveaux stress sous la forme du changement climatique, de la pollution atmosphériqueet de parasites envahissants. Détecter comment l'intensification de ces stress affectera la trajectoire des forêts est un défi scientifique majeur que ce document aborde. https://science.sciencemag.org/content/349/6250/ 814/tab-pdf

Changement climatique et phénomènes météorologiques (2000). Cette évaluation, parrainée par le Fonds mondial pour la nature, passe en revue les connaissances scientifiques sur le changement climatique et ses conséquences sur le temps, en particulier les phénomènes météorologiques extrêmes. Elle examine la mesure dans laquelle l'influence humaine sur le climat peut être mesurée, les attentes à court et à long terme et l'impact potentiel des mesures de réduction des émissions nettes de GES sur le climat futur. http://awsassets.panda.org/downloads/xweather.pdf

\section{5) RAVAGEURS ET MALADIES}

Guide de la lutte biologique classique contre les insectes nuisibles dans les forêts plantées et naturelles (2019). Cette publication de la FAO, fournit une théorie générale et des directives pratiques sur la lutte biologique classique, explique le «pourquoi» et le «comment» de la lutte biologique classique en foresterie, et aborde les risques associés à de tels programmes. http://www.fao.org/3/ca3677en/ca3677en.pdf

Guide pour l'application des normes phytosanitaires dans le secteur forestier (2011). Ce guide a pour but de contribuer à réduire la propagation des parasites facilitée par l'homme et ses impacts. Il fournit des informations faciles à comprendre sur les normes internationales relatives aux mesures phytosanitaires et sur le rôle des pratiques de gestion forestière dans la mise en œuvre des normes phytosanitaires et la facilitation de la sécurité des échanges commerciaux. www.fao.org/forestry/foresthealthguide/en/

Impacts du changement climatique sur la santé des (2008). Ce document passe en revue l'état actuel des connaissances sur les effets du changement climatique sur les ravageurs des forêts et leurs implications pour la protection et la gestion de la santé des forêts. En raison de la recherche relativement limitée qui a été consacrée spécifiquement aux parasites forestiers, des informations sur les parasites non forestiers sont également incluses pour permettre une meilleure compréhension des impacts potentiels du changement climatique sur la santé des forêts. http://www.fao.org/forestry/15905-odc804ee7d97e 656f06507bdcecddc721.pdf

\section{6) FEUX SAUVAGES}

Lignes directrices volontaires pour la gestion des incendies: principes et actions stratégiques (2006). Ces lignes directrices volontaires définissent un cadre de principes prioritaires qui aideront à la formulation de conditions politiques, juridiques, réglementaires et autres conditions favorables et d'actions stratégiques pour des approches plus holistiques de la gestion des incendies. Elles ont été conçues principalement pour les décideurs, les planificateurs et les responsables chargés de la gestion des incendies, y compris les États, le secteur privé et les ONG. Les directives couvrent les impacts sociaux, culturels, environnementaux et économiques positifs et négatifs des incendies naturels et planifiés dans les forêts, les zones boisées, les pâturages, les prairies et les paysages agricoles et ruraux/urbains. Le champ d'application comprend l'alerte précoce, la prévention, la préparation (à l'échelle internationale, nationale, infranationale et communautaire), une attaque initiale sûre et efficace des incendies et la restauration des paysages à la suite d'un incendie. http://www.fao.org/3/j9255e/j9255eoo.htm

Note de politique générale de la Banque mondiale: Managing Wildfires in a Changing Climate (2020). Cette note de politique générale vise à présenter les principaux messages et recommandations politiques pour atténuer les effets croissants des feux de forêt dans le monde et $s^{\prime} y$ adapter dans un contexte de changement climatique. Elle fait le point sur les facteurs qui contribuent aux feux de forêt extrêmes, notamment le changement climatique, le changement d'affectation des terres et les évolutions démographiques. Il recommande des mesures politiques qui peuvent être prises pour améliorer la prévention et la gestion des feux de forêt en fonction du contexte national. Le principal changement par rapport à l'approche réactive actuelle, qui repose souvent sur l'intervention d'urgence et la lutte contre les incendies, est l'augmentation des investissements 
dans la prévention. Les gouvernements peuvent contribuer à prévenir les incendies extrêmes par des mesures telles qu'une meilleure planification de l'utilisation des terres, l'élimination des incitations perverses à utiliser le feu pour modifier l'utilisation des terres et la clarification des droits de propriété foncière. D'autres actions incluent la mise en œuvre des techniques de gestion des incendies existantes, telles que la gestion intégrée des incendies et l'évaluation des risques d'incendie, l'amélioration de la surveillance et de la détection précoce des incendies et le renforcement de la participation des parties prenantes à la planification de la gestion des incendies.

Gestion des feux de forêt: manuel pour les formateurs (2010). Ce manuel soutient la mise en œuvre des lignes directrices volontaires pour la gestion des incendies, en ciblant les formateurs et les instructeurs sur le terrain. Avec les lignes directrices volontaires, le manuel constitue la base d'un programme de renforcement des institutions et des capacités en matière de gestion des incendies, en particulier dans les pays en développement. www.fao.org/docrep/012/i1363e/i1363eoo.htm

Incendies de forêt - Susciter des politiques de lutte contre les incendies dans l'UE (2018). Une analyse des connaissances, des méthodologies et des technologies produites au cours des deux dernières décennies ouvre de nouvelles perspectives pour la gestion des risques d'incendie de forêt face aux changements climatiques et environnementaux, aux tendances sociales et culturelles et aux dynamiques de croissance. Sur la base des conclusions de cette analyse, des recommandations clés sont proposées pour un dialogue plus approfondi entre les principaux acteurs afin d'améliorer la gestion des risques d'incendie de forêt en Europe. https://ec.europa.eu/info/sites/info/files/181116_ booklet-forest-fire-hd.pdf

Extrêmes du danger d'incendie de forêt en Europe dans le cadre du changement climatique: variabilité et incertitude (2017). Les forêts couvrent plus d'un tiers de la superficie totale de l'Europe. Ces dernières années, de grands incendies de forêt ont touché l'Europe à plusieurs reprises, en particulier les pays méditerranéens. Le risque d'incendie est influencé par les conditions météorologiques à court terme et par le climat lorsque l'on considère des intervalles de temps plus longs. Dans ce travail, l'accent est mis sur l'influence directe de la météo et du climat sur le risque d'incendie. https://publications.jrc.ec.europa.eu/repository/ bitstream/JRC108974/jrc108974_final.pdf
Changement climatique, séquestration du carbone et protection contre les incendies de forêt dans la zone boréale canadienne (2011). Ce rapport examine l'influence potentielle du changement climatique sur les régimes d'incendie dans les forêts boréales et les tourbières associées, ainsi que les implications pour la gestion des incendies dans ces écosystèmes. Il examine également le rôle des incendies de forêts boréales et de tourbières dans le cycle du carbone, à la fois par la libération de carbone (sous forme de GES) lors de la combustion et par l'absorption ultérieure de carbone dans le renouvellement de la végétation après un incendie. http://www.climateontario.ca/ MNR_Publications/stdprod_088316.pdf

Évaluation des risques d'incendie de forêt et stratégies innovantes pour la prévention des incendies (2010).(Cette publication présente les résultats de l'atelier sur l'évaluation des risques d'incendie de forêt et les stratégies innovantes pour la prévention des incendies, qui s'est tenu du 4 au 6 mai 2010 en Grèce. Elle passe en revue les systèmes nationaux actuels de prévention des incendies de forêt dans les pays européens et identifie les stratégies innovantes, les bonnes pratiques et les instruments politiques potentiels en matière de prévention des incendies de forêt en Europe. https://www.foresteurope.org/documentos/ FOREST_EUROPE_Forest_Fires_Report.pdf

\section{7) LES MANGROVES}

Les mangroves peuvent-elles suivre le rythme actuel de la montée du niveau de la mer? Une revue des données mondiales (2016). Cette publication passe systématiquement en revue les études publiées sur le changement du niveau de la surface (SEC) et du taux d'accrétion de la surface (SAR) à partir de sites de surveillance répartis dans le monde grâce à la méta-analyse et les compare aux scénarios SLR du Cinquième Rapport d'Evaluation du Groupe Intergouvernemental d'Experts sur le Changement Climatique (IPCC AR5). https://www.cifor.org/library/5775/

Les mangroves pour la défense des côtes. Directives pour les gestionnaires de côtes \& les décideurs politiques (2014). Ce guide pratique résume les découvertes des études et donne des recommandations pratiques de gestion destinées aux gestionnaires de zones côtières et aux décideurs politiques. Il permet au lecteur d'évaluer le contexte de risque dans une zone donnée, de définir des activités de gestion des risques liés aux mangroves et d'intégrer celles-ci 
dans des stratégies de réduction des risques, dans des protocoles d'adaptation au changement climatique et dans un plan d'aménagement côtier plus large. Des études de cas fournissent des exemples pratiques de méthodes de gestion des mangroves ainsi que des références relatives aux informations générales, aux outils pratiques d'évaluation des risques et de gestion des mangroves sont fournies tout au long du guide. https://www.nature.org/media/oceansandcoasts/ mangroves-for-coastal-defence.pdf

Les mesures pratiques pour lutter contre le changement climatique: les zones tampons des forêts côtières et le changement du littoral à Zanzibar, République-Unie de Tanzanie (2009). Ce rapport de l'Organisation des Nations Unies pour l'Education, la Science et la Culture présente les résultats des analyses (sur 50 ans) de changement des forêts côtières et des mesures pratiques pour atténuer les effets indésirables du ce changement. http://www.globalislands.net/userfiles/ Tanzania-11.pdf

La gestion des mangroves pour une résilience face au changement climatique (2006). Cet article donne un aperçu des écosystèmes de mangrove, des avantages des mangroves pour les populations ainsi que les menaces du fait de l'homme et de la planète qui compromettent les écosystèmes de mangrove. Il décrit les effets du changement climatique sur les mangroves et présente les outils et les stratégies pour renforcer la résilience des mangroves. https://portals.iucn.org/library/sites/library/files/ documents/2006-041.pdf

\section{8) LES COMMUNAUTÉS AUTOCHTONES/ LES MOYENS D'EXISTENCES}

Les droits des communautés locales et des populations autochtones sur les forêts d'Afrique Centrale: de l'espoir aux défis (2013). Cet article passe en revue les différents droits des communautés locales et des populations autochtones sur les ressources forestières en Afrique centrale. En 2010, le Conseil des Ministres de la Commission des Forêts d'Afrique Centrale (COMIFAC) a adopté les Directives Sous-régionales sur la Participation des Communautés Locales et des Populations Autochtones ainsi que des ONG à une gestion durable des forêts d'Afrique Centrale. Une enquête de cet instrument juridique sous-régional souligne un véritable engagement des États à consolider les avantages et les droits émergents à même d'améliorer les conditions de vie des communautés vulnérables et à renforcer le régime sous-régional de gestion durable des forêts. Cependant, l'efficacité des directives sous-régionales bute sur les lois administratives et les mesures pratiques des États membres visant à intégrer cet instrument dans leurs systèmes juridiques nationaux et le faire respecter. http://www.cifor.org/publications/pdf_files/ articles/AAssembe-Mvondo1301.pdf

Comprendre le REDD+ communautaire: un manuel pour les communautés autochtones (2011). Ce manuel préparé par le Groupe international de Travail pour les Affaires Autochtones et le Pacte des Peuples autochtones d'Asie est repose sur et cherche à promouvoir de manière holistique une approche du REDD+ qui respecte et promeut les Droits des Peuples autochtones tels que prévus dans la Déclaration des Nations Unies sur les Droits des Peuples Autochtones, des systèmes sociaux et culturels des peuples autochtones, de leurs valeurs et pratiques et de leur environnement. https://aippnet.org/wp-content/uploads/ 2020/02/21.-A-Manual-for-IndigenousCommunities-20120117174234.pdf

Un guide pour comprendre les impacts des projets REDD+ sur les moyens (2010). Ce guide donne un aperçu des méthodes de recherche qui permettent de cartographier les chaînes causales des projets REDD+ et recueille des données précieuses sur l'incidence des activités REDD+ sur le bien-être social des régions forestières. https://www.profor. info/sites/profor.info/files/CIFOR-learningREDD.pdf

\section{9) LE GENRE}

Les directives politiques de l'OIBT sur l'égalité des sexes et l'autonomisation des femmes (2018). L'OIBT est engagée en faveur de la parité hommesfemmes et à l'amélioration des résultats en matière d'égalité des genres dans sa politique et ses projets. Les directives politiques de l'OIBT sur l'égalité des genres et l'autonomisation des femmes (GEEW) institutionnalisent cet engagement. Les directives politiques servent de cadre à l'intégration du genre et de la parité hommes-femmes dans les politiques, les plans, les programmes, les projets, les activités et le fonctionnement interne de l'OIBT et visent à renforcer l'impact et l'efficacité des opérations de l'Organisation 
dans tous les domaines et à tous les niveaux. https://www.itto.int/direct/topics/topics_pdf_ download/topics_id=6107\&no=1\&disp=inline

Genre et Forêts: Le Changement Climatique, Tenure, Chaînes de Valeurs et Questions Emergentes (2016). Ce livre instructif compile les travaux de spécialistes du genre et de la foresterie d'horizons, de domaines de recherche et d'action divers afin d'analyser l'état du genre au niveau mondial en relation avec les forêts. A l'aide de diverses méthodes et approches, ils se sont appuyés sur un spectre de perspectives théoriques pour approfondir et élargir les questions pertinentes et aborder des thèmes actuels très peu étudiés. http://www.cifor.org/publications/pdf_files/Books/ BColfer1701.pdf

LeGenredansl'Agroforesterie:Numérospécial(2015). Il s'agit d'un numéro spécial de la Revue Internationale des Forêts sur le Genre dans l'Agroforesterie. Une liste complète des articles est disponible ici: https://www.cifor.org/library/5736/

Boîte à outils du genre: Un cadre d'analyse des rôles du genre dans la gestion forestière (2012). Cet article tente de fournir des directives ciblées tout en reconnaissant l'incertitude généralisée quant à la manière d'aborder la question du genre dans le monde de la foresterie (par des chercheurs et des professionnels des ressources naturelles, du développement et de la conservation). Les méthodes de genre y sont divisées en trois approches principales basées sur la disponibilité des ressources. http://www.cifor. org/publications/pdf_files/OccPapers/OP-82.pdf

\section{0) LES SERVICES ÉCOSYSTÉMIQUES}

La valorisation des services écosystémiques des forêts: Un manuel de formation pour les planificateurs et les développeurs de projets (2019). Ce manuel publié par la FAO est conçu comme un outil de formation destiné aux agents et aux professionnels du terrain travaillant dans les agences environnementales et forestières et dans d'autres secteurs gouvernementaux concernés. Il porte sur les forêts et autres écosystèmes arboricoles du Bangladesh. Cependant, les concepts, méthodes et approches qui y sont décrits peuvent être appliqués dans diverses situations. Le public cible de ce manuel comprend ceux qui doivent examiner les coûts et les bénéfices environnementaux des projets de développement mais ne disposent pas nécessairement d'une solide formation en économie de l'environnement. L'objectif est d'asseoir une solide connaissance des services écosystémiques et de leur évaluation économique à travers une approche progressive. Le manuel explique les concepts sous-jacents, donne des définitions, énonce les principes des mathématiques financières et de l'évaluation économique et donne des exemples et des exercices.

http://www.fao.org/3/ca2886en/CA2886EN.pdf

Les forêts et l'eau - Évaluation et paiements des services écosystémiques forestiers (2018). Les objectifs de cette étude menée par la FAO et la CEEONU consistent à améliorer la compréhension des moyens par lesquels les systèmes de paiement des services écosystémiques peuvent être appliqués aux forêts, notamment en se focalisant sur les fonctions hydrologiques des forêts pour l'intérêt mutuel des hommes et de l'environnement. En outre, l'étude traite des progrès et des défis auxquels ces systèmes sont confrontés et donne une orientation pratique aux décideurs politiques et aux professionnels. Cette étude renferme la base de données la plus complète actuellement disponible d'études de cas sur les systèmes de paiement liés à l'eau pour les services écosystémiques forestiers dans la zone CEE-ONU. https://www.unece.org/fileadmin/DAM/timber/ publications/sp-44-forests-water-web.pdf

Les Services Ecosystémiques Forestiers (2018). Une Étude analytique préliminaire préparée pour la treizième session du Forum des Nations Unies sur les Forêts. https://www.un.org/esa/forests/wpcontent/uploads/2018/05/UNFF13_BkgdStudy ForestsEcoServices.pdf

Les paiements des services environnementaux des forêts en Afrique subsaharienne: Un guide pratique (2016). L'objectif à long terme de cette publication de la FAO est d'augmenter les ressources financières pour une gestion forestière durable en améliorant la reconnaissance et la valorisation des bénéfices procurés par les arbres, les forêts et la foresterie. À ce titre, cette publication donne des détails sur diverses stratégies de mobilisation des investissements pour la conservation et l'augmentation des valeurs économiques, sociales et environnementales des forêts tout en renforçant les bénéfices pour les communautés africaines. En partageant des exemples de réussite, la publication démontre comment des incitations économiques adaptées peuvent 
permettre le transfert de ressources financières des bénéficiaires des services environnementaux vers les fournisseurs de ces services. Elle cherche également à donner aux gouvernements nationaux les moyens de jouer un rôle de catalyseur pour garantir la prise en compte sur le marché de toute la gamme des valeurs économiques des forêts. http://www.fao.org/3/a-i5578e.pdf

Les Paiements des Services Ecosystémiques Forestiers: Analyse SWOT et Possibilités de Mise en œuvre (2016). L'objectif de ce rapport est de présenter une vue d'ensemble de la mise en œuvre des PSE dans les écosystèmes forestiers en Europe. Une analyse SWOT est présentée sur les bases suivantes: premièrement, l'analyse des facteurs politiques liés aux PSE au niveau de l'UE, deuxièmement, une analyse documentaire et enfin une comparaison des meilleures pratiques en matière de PSE. Les meilleures pratiques des pays européens sont présentées dans les études de cas des systèmes de PSE établis et fonctionnels. https://publications.jrc.ec.europa.eu/repository/ bitstream/JRC103176/lb-na-28128-en-n.pdf

Comment la Gestion Forestière affecte les Services Écosystémiques, notamment la Production de Bois et la Rentabilité Économique: Synergies et Compromis (2012).Cette publication utilise des séries de données virtuelles mais réalistes pour quantifier, dans le cas de services multiples, les effets de cinq alternatives de gestion forestière qui forment un gradient d'intensité. https://www.jstor.org/stable/pdf/26269223.pdf? refreqid=excelsior\%3A6fde 221457 eabd 24 gefd277e998ee971

Les incitations au renforcement des services écosystémiques des forêts: bilan et enseignements pour le REDD (2009). Cette publication présente un résumé d'une revue de la conception et des performances de paiements choisis pour les services écosystémiques et d'autres initiatives incitatives dans quatre grandes régions de forêts tropicales - le Bassin de l'Amazone, le Bassin du Congo, les forêts de Miombo d'Afrique orientale et australe et ceux d'Asie du Sud-Est. Le rapport met l'accent sur un certain nombre de questions essentielles pour la conception et la mise en œuvre des activités REDD nationales et infranationales en mettant l'accent sur le rôle des mécanismes d'incitation basés sur la performance et les conditions essentielles pour leur mise en œuvre efficace.

https://pubs.iied.org/pdfs/13555IIED.pdf

\section{1) L'EAU}

WaPOR: Le portail de la FAO sur la productivité de l'eau à travers un accès libre à des données dérivées de la télédétection surveille et rend compte de la productivité de l'eau à usage agricole en Afrique et au Proche-Orient. Il s'agit d'un nouvel outil essentiel pour faire face à la pénurie d'eau et s'adapter à l'évolution des conditions météorologiques. www.fao.org/in-action/remote-sensing-for-waterproductivity/wapor\#/home

La Forêt et l'Eau sur une Planète en Mutation: Vulnérabilité, Adaptation et Opportunités de Gouvernance. Un Rapport d'Evaluation Globale (2018). Ce rapport complet présente les résultats de la 6e évaluation scientifique faite dans le cadre de l'initiative des Panels Mondiaux d'Experts Forestiers (GFEP). Le Panel Mondial d'Experts sur les Forêts et l'Eau co-présidé par Irena F. Creed et Meine van Noordwijk a préparé un rapport de synthèse scientifique et la note stratégique qui va avec et qui a été lancée lors du Forum Politique de Haut Niveau des Nations Unies sur le Développement Durable de 2018. Plus de 50 scientifiques de 20 pays ont contribué à cette évaluation majeure du lien entre les forêts, l'eau, le climat et les populations. Le principal contexte politique mondial de cette évaluation est défini par les 17 objectifs de développement durable (ODD). L'eau est au cœur de ces 17 objectifs et les forêts sont indissociablement liées à l'eau. Le rapport reflète l'importance d'une action intégrée visant à garantir l'accès à l'eau pour tous et à préserver la vie sur terre et souligne les liens importants entre l'eau et les forêts. https://www.iufro.org/fileadmin/material/ publications/iufro-series/ws38/ws38.pdf

La gestion forestière et son impact sur les ressources en eau: une étude portant sur 13 pays (2017). Cette publication de l'UNESCO donne des résumés par pays de l'état actuel des connaissances sur la relation entre la gestion forestière et les ressources en eau. Les chapitres ont été soumis par des scientifiques de 13 pays qui sont témoins des effets de la gestion forestière sur l'eau. La publication cherche à identifier les similitudes et les différences dans l'historique de la gestion forestière, le climat, les types de végétation et les conditions socio-économiques qui ont conduit à l'utilisation et à la gestion des forêts. Le contenu principal de ce rapport vise à clarifier la manière avec laquelle la coopération et les conflits autour des forêts et 
des ressources en eau se multiplient dans le monde. Les recherches futures devraient se focaliser sur la manière de résoudre les conflits actuels et futurs en tenant compte des climats locaux. https://unesdoc.unesco.org/ark:/48223/pfooo0247902

L'Eau, le changement climatique et les forêts: la gestion des bassins hydrographiques pour un climat en mutation (2010). Ce rapport décrit les possibilités d'adaptation liées à la gestion forestière, en particulier dans le contexte de l'eau et des écosystèmes aquatiques. Les deux premières sections décrivent l'importance des forêts pour les ressources en eau aux États-Unis d'Amérique et résument les effets observés et escomptés du changement climatique sur le cycle hydrologique et les bassins hydrographiques forestiers.

www.fs.fed.us/pnw/pubs/pnw_gtr812.pdf

Les Forêts et l'Eau (2008). Cette publication de la FAO explique le rôle des forêts dans le cycle hydrologique en mettant particulièrement l'accent sur les zones forestières présentant un risque critique telles que les terrains montagneux ou escarpés, les zones fluviales et côtières et les écosystèmes marécageux. Elle aborde également la protection des approvisionnements municipaux en eau et les systèmes émergents de paiement des services de bassins hydrographiques. La publication met en évidence la nécessité d'une gestion holistique dans les écosystèmes de bassins hydrographiques complexes en tenant compte des interactions entre l'eau, les forêts et les autres utilisations des terres, ainsi que les facteurs socio-économiques. http://www.fao.org/forestry/43810-05bc28890480b 481d4310a3c5fe8a1003.pdf

La nouvelle génération de programmes et de projets de gestion de bassins hydrographiques (2006). Cette publication de la FAO a été principalement produite à l'intention des professionnels du terrain chargés de la gestion des bassins hydrographiques et des décideurs locaux impliqués dans la gestion des bassins hydrographiques au niveau district ou municipal. Elle fournit des informations sur la nouvelle génération de gestionnaires de bassins hydrographiques en utilisant des exemples de la France, de l'Italie, du Kenya, du Népal et du Pérou. www.fao.org/docrep/009/a0644e/a0644eoo.htm

\section{2) LA BIODIVERSITÉ}

La faune dans un contexte de changement climatique (2012). Cette publication de la FAO analyse et présente la manière avec laquelle le changement climatique affecte ou affectera certainement les animaux sauvages et leurs habitats. Même si le changement climatique a déjà été observé et suivi pendant plusieurs décennies, il existe peu d'études poussées sur comment le phénomène affecte la faune. Toutefois, il est de plus en plus évident que le changement climatique exacerbe considérablement d'autres pressions majeures d'origine humaine telles que l'empiètement, la déforestation, la dégradation des forêts, le changement d'affectation des terres, la pollution et la surexploitation des ressources fauniques. Des études de cas sont présentées. Celles-ci décrivent certains éléments de l'ensemble des données dans certains cas et fournissent des projections de scénarios probables dans d'autres. www.fao.org/forestry/30143-obb7fb87ece780936a2f 55130c87caf46.pdf

Le changement climatique et les ressources génétiques forestières: l'état des connaissances, des risques et des opportunités (2011). Cet article de la FAO passe en revue et examine les effets du changement climatique sur les ressources génétiques des organismes qui sont importantes pour le bien-être de l'homme et le rôle potentiel de ces ressources dans l'atténuation et l'adaptation au changement climatique. Il se focalise sur les ressources génétiques forestières dans le contexte des arbres des forêts naturelles, des plantations et des systèmes agroforestiers. L'article est une revue de l'état actuel des connaissances et une identification des insuffisances ainsi que les priorités d'action. www.fao.org/docrep/meeting/023/mb696e.pdf

Le changement climatique et les ressources forestières et fauniques africaines (2011). Ce manuel préparé par le Forum Forestier Africain présente systématiquement le changement climatique dans le contexte des forêts, des arbres et des ressources fauniques africains. Il contient des informations sur les aspects plus globaux du changement et de la variabilité climatique, un aperçu du changement climatique sur les ressources fauniques africaines et des considérations socio-économiques et politiques afin d'aborder les questions liées au changement climatique dans le secteur forestier. http://afforum.org/sites/default/files/English/ English_o.pdf 
Les systèmes agroforestiers européens améliorentils la biodiversité et les services écosystémiques? Une méta-analyse (2016). Une analyse des effets de l'agroforesterie sur la biodiversité et les services écosystémiques qui montre que l'agroforesterie a un effet globalement positif mais qu'il s'agit d'un effet dépendant du contexte. https://www.sciencedirect.com/science/article/pii/ S0167880916303097

Le REDD+ et la biodiversité (2011). Ce document a été élaboré par le Secrétariat de la Convention sur la diversité biologique (CDB) en vue de fournir des informations techniques et scientifiques sur la conception et la mise en œuvre des activités du REDD+ d'une manière qui ne soit pas contraire aux objectifs de la CDB et qui soutienne la mise en œuvre du programme de travail sur la biodiversité forestière. Plus spécifiquement, le document vise à souligner: les bénéfices potentiels du REDD+ pour la biodiversité et les communautés autochtones et locales, l'importance de la biodiversité et des avantages connexes pour un succès durable du REDD+, les éventuels risques du REDD+ pour la biodiversité et les communautés autochtones et locales en vue de contribuer au développement ou à l'amélioration des recommandations politiques appropriées, les moyens par lesquelles la CDB peut contribuer au succès du REDD+ et les potentiels voies à travers lesquelles le REDD + peut contribuer aux objectifs de la CDB.

www.cbd.int/doc/publications/cbd-ts-59-en.pdf

La gestion forestière, la biodiversité et les moyens d'existence durables: un guide de bonnes pratiques (2009). Cette publication du Secrétariat de la CDB traite des liens entre la foresterie, la biodiversité et le développement/la réduction de la pauvreté. Les synthèses et les exemples montrent comment la biodiversité et le développement économique durable peuvent faire bon ménage. wWw.cbd.int/development/doc/cbd-goodpractice-guide-forestry-booklet-web-en.pdf

La biodiversité des montagnes et le changement climatique (2009). Cette publication du Centre International pour le Développement Intégré des Montagnes examine les effets du changement climatique sur les écosystèmes de montagne et les conséquences pour le reste du monde. Elle traite de la situation actuelle et des approches en vue d'une future stratégie commune pour la conservation de la biodiversité des montagnes. http://lib.icimod.org/ record/7973/files/attachment_613.pdf

La résilience des forêts, la biodiversité et le changement climatique: une synthèse de la relation biodiversité/résilience/stabilité dans les écosystèmes forestiers (2009). Ce document du Secrétariat de la CDB passe en revue les concepts de résilience, de résistance et de stabilité des écosystèmes forestiers et leur relation avec la biodiversité en faisant particulièrement référence au changement climatique. https://www.cbd.int/doc/publications/cbd-ts-43en.pdf

Les directives d'OIBT/UICN pour la conservation et l'utilisation durable de la biodiversité dans les forêts de production de bois tropicaux (2009). Ces directives ont été élaborées par l'OIBT et l'Union Internationale pour la Conservation de la Nature et distinguent deux niveaux d'intervention. À un premier niveau, les directives définissent les approches générales en matière de gestion forestière qui auront une large application pour garantir le maintien des valeurs de la biodiversité et qui devraient être adoptées partout. Au second niveau, elles passent en revue les expériences pratiques et fournissent des conseils que les gestionnaires et les décideurs pourraient utiliser pour concevoir des directives, des codes de pratique, des règlements et des pratiques sylvicoles applicables au niveau local. https://www.itto.int/direct/topics/topics_pdf_ download/topics_id=1918\&no=0\&disp=inline

Relier la biodiversité à l'atténuation et à l'adaptation au changement climatique: le rapport du deuxième groupe d'experts technique ad hoc sur la biodiversité et le changement climatique (2009). Ce document de la CDB examine les effets observés et escomptés du changement climatique sur la biodiversité, les liens entre la biodiversité et l'adaptation et l'atténuation du changement climatique, les liens entre la biodiversité et l'atténuation des effets du changement climatique en mettant l'accent sur les activités d'utilisation des terres et la réduction des émissions dues à la déforestation et à la dégradation des forêts et donne des informations sur les techniques de valorisation de la biodiversité. Il souligne que l'application de ces techniques permet de quantifier les coûts et les bénéfices, les opportunités et les défis et par conséquent l'amélioration de la prise de décision concernant les activités liées au changement climatique.

www.cbd.int/doc/publications/cbd-ts-41-en.pdf 
Le changement climatique et la diversité génétique des forêts: les implications pour une gestion forestière durable en Europe (2007). La publication passe en revue les connaissances actuelles sur la manière avec laquelle les arbres forestiers feront face et s'adapteront au changement climatique. Elle traite également des implications pour la SFM en Europe. WwW.euforgen.org/fileadmin/bioversity/ publications/pdfs/1216.pdf

Le suivi écologique de la gestion forestière dans les tropiques humides: un guide pour les opérateurs et les certificateurs forestiers avec un accent mis sur les forêts à haute valeur de conservation (2004). Cette publication donne des directives à l'effort mondial pour une gestion durable des forêts à haute valeur de conservation (FHVC) en utilisant des critères valables de production et de conservation. L'objectif général est de fournir aux opérateurs et aux certificateurs de forêts tropicales des procédures méthodologiques pratiques et adaptées au suivi écologique des activités forestières certifiées dans le cadre des FHCV (selon les protocoles et les normes du Forest Stewardship Council). https:// www.yumpu.com/en/document/read/21765940/ ecological-monitoring-of-forestry-managementin-the-humid-tropics-

\section{3) LA SÉCURITÉ ALIMENTAIRE ET NUTRITIONNELLE}

Tester les Diverses Voies liant le Couvert Forestier et la Diversité Alimentaire dans les Paysages Tropicaux (2019). Un régime alimentaire diversifié est important pour lutter contre les carences en micronutriments et les autres formes de malnutrition qui constituent l'un des plus grands défis des systèmes alimentaires modernes. Dans les pays tropicaux, plusieurs études ont établi une association positive entre le couvert forestier et la diversité alimentaire même si les mécanismes réels de cette association restent encore à identifier et à quantifier. Trois voies complémentaires qui pourraient lier les forêts aux régimes alimentaires sont explorées dans cet article: une voie directe (par exemple, la consommation de nourriture forestière), une voie de revenus (revenus provenant des produits forestiers utilisés pour acheter de la nourriture sur les marchés) et une voie agroécologique (forêts et arbres renforçant la production agricole). https:// w w w.cifor.org/library/7441/
La structure des forêts, et pas seulement la quantité, influence la qualité de l'alimentation dans cinq pays africains (2019). Cet article fait le lien entre les mesures forestières et les données sur la consommation alimentaire dans cinq pays africains. https://www.sciencedirect.com/science/ article/pii/S2211912419300021?via\%3Dihub

Une foresterie durable pour la sécurité alimentaire et la nutrition: Un rapport du Panel d'Experts de Haut Niveau sur la Sécurité Alimentaire et la Nutrition (2017). Ce rapport appelle à une connaissance réactualisée de la foresterie durable afin d'intégrer pleinement les différentes fonctions des forêts et des arbres, de la ferme et du paysage jusqu'au niveau mondial ainsi qu'à différentes échelles temporelles pour une meilleure sécurité alimentaire et nutritionnelle et un développement durable. Cela nécessite des mécanismes de gouvernance inclusifs et intégrateurs à différentes échelles qui permettent la participation pleine et effective des acteurs concernées notamment des populations autochtones et des communautés locales qui dépendent des forêts. http://www.fao. org/fileadmin/user_upload/hlpe/hlpe_documents/ HLPE_Reports/HLPE-Report-11_EN.pdf

Les Forêts et l'Alimentation: Les facteurs déterminants des forêts et des systèmes arboricoles pour la Sécurité Alimentaire et la Nutrition (2015). Une revue des résultats scientifiques indique douze grands facteurs déterminants de la sécurité alimentaire et nutritionnelle dans le contexte des forêts (à savoir la croissance démographique, l'urbanisation, les changements de gouvernance, le changement climatique, la commercialisation de l'agriculture, l'industrialisation des ressources forestières, les déséquilibres en matière de genre, les conflits, la formalisation des droits fonciers, la hausse des prix des denrées alimentaires et l'augmentation du revenu par habitant). https://www.cifor.org/library/5937

\section{4) LA GESTION FORESTIIERE}

L'Instrument des Nations Unies sur les forêts. Fournit un cadre d'action national et de coopération internationale pour une gestion durable des forêts. https://www.un.org/esa/forests/wp-content/ uploads/2018/08/UN_Forest_Instrument.pdf

Le Plan stratégique des Nations unies sur les forêts, 2017-2030. Un outil pour appuyer les efforts des 
pays pour l'intégration des services écosystémiques forestiers dans tous les aspects liés à l'élaboration de leurs politiques et planification nationales. https://www.un.org/esa/forests/wp-content/ uploads/2016/12/UNSPF_AdvUnedited.pdf

La Boîteà Outils pour une Gestion Durable des Forêts (GDF). Il rassemble un nombre important d'outils, d'études de cas et d'autres ressources classées sous forme de modules. Il a été créé pour permettre aux propriétaires et aux gestionnaires des forêts ainsi qu'à d'autres acteurs d'accéder facilement à ces ressources pour la mise en œuvre de la GDF. http://www.fao.org/sustainable-forestmanagement/toolbox/en/

Le Programme sur les Forêts (PROFOR). Un partenariat multi-donateurs qui fournit des connaissances, des outils et des analyses approfondies pour faciliter la contribution des forêts à la réduction de la pauvreté, au développement économique durable et à la protection des services environnementaux au niveau mondial et local. https://www.profor.info/tools

Agtrials: Le Répertoire et la Base de Données des Essais Agricoles à l'Echelle Mondiale. Un portail d'information conçu par le programme de recherche du CGIAR sur le changement climatique, l'agriculture et la sécurité alimentaire (CCAFS) donnant accès à une base de données qui recueille et analyse les performances des technologies agricoles sous l'effet du changement climatique dans les pays en développement. Il est destiné à aider à évaluer davantage et à améliorer les modèles de production agricole dans les conditions climatiques actuelles et futures. http://www.agtrials.org/

Les directives de la FAO sur la foresterie urbaine et périurbaine (2016). Elles sont destinées à fournir un cadre de référence aux décideurs et aux planificateurs pour planifier, concevoir et gérer de manière adéquate la forêt et les arbres dans leurs villes et aux alentours. Le document comprend un aperçu général du rôle des forêts urbaines et périurbaines pour un développement urbain durable ainsi que des chapitres spécifiques recommandant des actions politiques et de gestion à prendre pour maximiser leur contribution à la prise en charge des défis mondiaux et locaux notamment le changement climatique. http://www.fao.org/3/a-i6210e.pdf
Les directives volontaires pour la gestion durable des forêts tropicales naturelles (2015). Cette publication est une mise à jour du premier document d'orientation politique de l'OIBT sur la gestion des forêts tropicales naturelles publié en 1990. Les nouvelles directives volontaires s'appuient sur des connaissances accrues et sur l'émergence d'un large éventail de nouveaux défis et d'opportunités pour la gestion des forêts tropicales. Il est conçu pour servir de ligne directrice dans la prise en charge des questions politiques, juridiques, de gouvernance, institutionnelles, écologiques, sociales et économiques qui doivent être prises en compte dans la planification, la mise en œuvre et l'évaluation de la GDF dans les forêts tropicales naturelles afin d'assurer la fourniture durable de biens forestiers et de services environnementaux. https://www.itto.int/direct/topics/topics_pdf_ download/topics_id=4330\&no=0\&disp=inline

Les directives sur la gestion durable des forêts dans les zones arides d'Afrique subsaharienne (2010). Ces directives élaborées par la FAO visent à aider les décideurs et les gestionnaires des forêts à prioriser les questions et les aspects qui doivent être abordés pour l'amélioration de la planification et de la gestion des forêts dans les zones arides et contribuer ainsi au bien-être des populations locales et à l'amélioration de leurs avantages sociaux, culturels, environnementaux et économiques. http://www.fao.org/3/i1628e/i1628eoo.pdf

Les directives volontaires pour la gestion responsable des forêts plantées (2006). Ces directives de la FAO, qui sont non contraignantes juridiquement, peuvent être adoptées et appliquées aux forêts plantées dans toutes les zones écogéographiques et dans les pays, régions et paysages à tous les stades du développement économique. Elles sont applicables aux forêts plantées qui remplissent des fonctions productives pour la fourniture de bois, de fibres et de produits forestiers non ligneux ou des fonctions protectrices pour la fourniture de services écosystémiques et sociaux. Elles couvrent tous les aspects des forêts plantées depuis l'élaboration et la planification des politiques jusqu'aux considérations techniques relatives à la gestion des forêts plantées. Les directives établissent un cadre pour favoriser le dialogue dans la formulation des politiques, des lois, des règlements et des plans stratégiques et de gestion qui, à leur tour, contribueront à l'amélioration des conditions et au renforcement des capacités et des compétences 
en matière de gestion des forêts plantées. http://www.fao.org/3/j9256e/j9256eoo.pdf

Le manuel de foresterie durable: un guide pratique pour les gestionnaires de forêts tropicales sur la mise en ouvre de nouvelles normes (2004). Ce manuel constitue un guide clair et concis sur les aspects pratiques de la mise en œuvre de normes internationales en matière de GDF. Il s'adresse aux gestionnaires des forêts et leur explique en termes clairs les exigences des normes ainsi que la voie à suivre pour les mettre en œuvre. https://www.taylorfrancis.com/books/9781849773317

Les directives de l'OIBT pour la restauration, la gestion et la réhabilitation des forêts tropicales dégradées et secondaires (2002). Ces directives de l'OIBT soulignent l'importance croissante des forêts dégradées et secondaires dans les paysages tropicaux. Elles présentent un ensemble de principes et d'actions recommandées pour promouvoir et encourager la gestion, la restauration, la réhabilitation et l'utilisation durable des forêts dégradées et secondaires comme une composante du développement socio-économique durable. https://www.itto.int/direct/topics/topics_pdf_ download/topics_id=1540000\&no=1\&disp=inline

Le code modèle FAO des pratiques d'exploitation forestière (1996). Le code modèle FAO vise à promouvoir des pratiques d'exploitation qui vont améliorer les normes d'utilisation des forêts, réduire les incidences environnementales, contribuer à assurer la pérennité des forêts pour les générations futures et améliorer les contributions socio-économiques de la foresterie en tant que composante du développement durable. Il examine la planification de la récolte, la mise en œuvre et le contrôle des opérations de récolte, l'évaluation de la récolte, la communication des résultats à l'équipe de planification et le développement d'une main-d'œuvre compétente et dûment motivée. Des informations sont également fournies sur les éventuelles conséquences qui pourraient découler d'un échec dans la mise en œuvre de ces pratiques. www.fao.org/docrep/V6530E/V6530E0o.htm

Les critères et indicateurs pour la gestion durable des forêts tropicales (2016).Cette publication est une mise à jour des Critères de l'OIBT relatifs à la Mesure de la Gestion Durable des Forêts Tropicales. Ces critères ont été élaborés pour aider dans le suivi et l'évaluation de l'état des forêts tropicales naturelles dans les pays membres producteurs de l'Organisation et pour identifier les besoins d'amélioration des pratiques forestières. https://www.itto.int/direct/topics/topics_pdf_ download/topics_id=4872\&no=1\&disp=inline

\section{5) L'ADAPTATION}

PNA -Ag Réservoir de Connaissances. Cette base de données consultable aide les pays à mettre en évidence les ressources clés nécessaires à la planification et à la budgétisation de l'adaptation et plus particulièrement dans l'élaboration et la mise en œuvre des plans nationaux d'adaptation (PNA ). Elle contient des outils, des méthodes, des études de cas et d'autres supports de connaissances sur l'adaptation au changement climatique dans les secteurs agricoles (cultures, élevage, foresterie, pêche et aquaculture). Cet outil est destiné aux planificateurs nationaux, aux acteurs de développement et aux décideurs. http://www.fao. org/in-action/naps/knowledge-tank/en/

L'Adaptabilité des principales espèces agroforestières d'Amérique Centrale aux climats futurs (2017). Cet atlas (de Souza et al., 2017) présente des cartes d'adaptation actuelles et futures pour 54 espèces qui sont fréquemment utilisées comme ombrage dans les systèmes agroforestiers en Amérique centrale. Les 54 espèces d'arbres qui ont été sélectionnées comprennent 24 espèces d'arbres fruitiers, 24 arbres de bois d'œuvre et six espèces utilisées dans l'amélioration de l'état du sol. http://www.worldagroforestry.org/atlas-centralamerica

Le Conseil Canadien des Ministres des Forêts (CCMF), une série d'adaptation au changement climatique.Le CCMF a identifié le changement climatique comme l'une des deux questions prioritaires pour le secteur forestier Canadien. Les ministres en charge de la gestion forestière ont été invités à collaborer avec le gouvernement fédéral sur l'adaptation dans le secteur forestier via le Groupe de Travail sur le Changement Climatique du CCMF. La phase 1 de ce travail, achevée en 2010, a consisté en une évaluation complète de la vulnérabilité de diverses espèces d'arbres et a permis de définir des options de gestion pour une adaptation. La phase 2 est allée au-delà des arbres pour examiner l'adaptation au changement climatique au sein des écosystèmes forestiers et du secteur forestier en général. Les résultats de ces phases sont repris dans la série Adaptation au 
changement climatique du CCMF qui, comprend plusieurs rapports techniques et documents de synthèse.

https://www.ccfm.org/english/coreproducts-cc.asp

MOTIVE: Le projet sur les modèles de gestion forestière adaptative. MOTIVE étudie les stratégies de gestion adaptative qui prennent en charge le changement climatique et l'utilisation des terres. Il examine également les effets de ces changements par rapport à une large gamme de biens forestiers et de services écosystémiques.

http://motive.pensoft.net/

L'Adaptation au climat: la plateforme européenne d'adaptation au climat (CLIMATE-ADAPT). Cette plateforme a pour but d'aider l'Europe á s'adapter au changement climatique, particulièrement en fournissant un accès et des informations sur les changements climatiques attendus, la vulnérabilité présente et future des régions et secteurs, notamment la foresterie, les stratégies d'adaptation national et internationales et les potentielles options d'adaptation.

https://climate-adapt.eea.europa.eu/

weADAPT, l'explorateur du changement climatique. Il s'agit d'une plateforme en ligne axée sur l'adaptation (et les synergies entre l'adaptation et l'atténuation) qui s'adresse aux professionnels, aux chercheurs et aux décideurs en leur proposant un accès aux informations et des possibilités de partager les expériences et les leçons apprises. http://weadapt.org/

Le développement récent de la recherche en agroforesterie et son rôle dans l'adaptation et l'atténuation du changement climatique (2016). Cet article passe en revue le récent développement technologique du modèle agroforestier et son rôle dans les services environnementaux notamment dans la séquestration du carbone. https://www. researchgate.net/profile/Om_Prakash_Chaturvedi/ publication/304879691_Recent_development_ in_agroforestry_research_and_its_role_in_ climate_change_adaptation_and_mitigation/ links/577c9ff208aece6c2ofcd9a1/Recentdevelopment-in-agroforestry-research-andits-role-in-climate-change-adaptation-andmitigation.pdf

Les effets du changement climatique et adaptation de la gestion forestière: une revue (2015). Cet article effectue une revue de la littérature qui se trouve dans la base de données du Web of Science et qui porte sur les impacts du changement climatique sur les forêts et les options d'adaptation pour la gestion forestière. La revue documentaire, qui se concentre sur les articles et les rapports publiés entre 1945 et 2013, identifie des thèmes clés pour les chercheurs et les gestionnaires des forêts. https://link.springer. com/article/10.1007/s13595-014-0446-5

La gestion des forêts comme des systèmes adaptatifs complexes: le renforcement de la résilience face au défi de l'évolution mondiale (2013). Ce manuel fait le lien entre les concepts émergents de complexité, les systèmes complexes d'adaptation et de résilience à l'écologie et à la gestion forestières. Il explore la manière avec laquelle ces concepts peuvent être appliqués dans divers biomes forestiers avec leurs différents cadres écologiques, socio-économiques et historiques. Certains chapitres mettent l'accent sur différents éléments de ces concepts en fonction du cadre spécifique et de l'expertise des auteurs. Les régions et les auteurs ont été sélectionnés pour couvrir une diversité de points de vue et de spécialités, allant de la foresterie et des forêts naturelles à la restauration des forêts ainsi que des forêts boréales aux forêts tropicales.

www.routledge.com/books/details/9780415519779/

L'Adaptation aux risques climatiques par les petits exploitants agricoles: le rôle des arbres et de l'agroforesterie (2013).Cet article, qui s'appuie sur des exemples au niveau mondial avec un accent sur des études de cas africaines, illustre les rôles variés des arbres et de l'agroforesterie dans la réduction de l'exposition des petits exploitants aux risques liés au climat. https://www.sciencedirect.com/science/ article/pii/S1877343513001619?via\%3Dihub

Revue de la science et de la mise en ouvre des mesures d'adaptation au changement climatique dans le secteur forestier Européen (2011). Cet article présente une revue complète des potentiels options d'adaptation dans le secteur de la foresterie en Europe. La revue repose sur trois piliers: une revue de la littérature scientifique, une analyse des stratégies de réponse nationales actuelles et une évaluation d'experts fondée sur une base de données compilée dans «COST Actions ECHOES» (Changement Climatique Attendu et Options pour la foresterie Européenne). Les mesures d'adaptation englobent des réponses aux risques et aux opportunités créés par le changement climatique et prennent en charge toutes les étapes des 
opérations forestières. Les mesures visant à réduire la vulnérabilité au changement climatique peuvent soit viser à réduire la sensibilité des forêts face aux effets néfastes du changement climatique, soit à accroître la capacité d'adaptation pour faire face aux conditions environnementales en mutation. https://www.mdpi.com/1999-4907/2/4/961

Les réponses au changement climatique dans les forêts nationales: un manuel pour l'élaboration d'options d'adaptation (2011).Ce manuel est un condensé des connaissances actuelles sur l'adaptation au changement climatique. Cellesci sont tirées de synthèses pédagogiques, d'outils spécifiques, de dialogues facilités, d'ateliers et d'études de cas. Il se focalise spécifiquement sur des sujets et approches qui sont adaptés et compatibles avec la gestion des ressources forestières nationales et potentiellement sur d'autres terres fédérales. Le manuel est destiné à faciliter la transition vers des approches adaptées au climat dans la gestion des ressources. Il ne prétend pas être un compte-rendu exhaustif de tous les efforts scientifiques et de gestion en matière d'adaptation au changement climatique mais plutôt une compilation d'informations et de leçons apprises qui serviront de base à la planification et à la pratique de l'adaptation dans les forêts nationales. www.fs.fed.us/pnw/pubs/pnw_gtr855.pdf

La foresterie européenne face au changement climatique: les directives EUSTAFOR (2010). L'objectif de cet article est de fournir des orientations aux membres de l'EUSTAFOR sur la manière par laquelle les forêts peuvent être gérées pour offrir un maximum d'avantages en termes d'atténuation et d'adaptation au changement climatique. Les directives forment un cadre commun de recommandations pour les membres de l'EUSTAFOR se focalisant sur l'équilibre du carbone. Elles s'inscrivent dans le cadre des directives Paneuropéennes de niveau opérationnel pour la GDF. Ces directives sont volontaires et ont été élaborées pour les forêts étatiques. Elles appuient les synergies dans la mise en œuvre du Forum des Nations Unies sur les forêts, de la CCNUCC, de la Convention sur la diversité biologique, de la Convention des Nations Unies sur la Lutte contre la Désertification et d'autres engagements internationaux applicables aux forêts. https://eustafor.eu/uploads/ EustaforClimateReport2010_2-1.pdf
L'Adaptation des forêts au changement climatique: quelques estimations (2010). Cet article se penche sur les effets escomptés du changement climatique sur les forêts en général et sur la production industrielle de bois en particulier. Il présente des études de cas du Brésil, de l'Afrique du Sud et de la Chine. L'objectif est d'identifier les probables dommages et les investissements ou activités d'atténuation possibles. Les résultats fournissent une perspective, des estimations et des projections des effets du changement climatique sur les forêts et la foresterie dans plusieurs régions et pays. http://www.indiaenvironmentportal.org. in/files/Adaptation $\% 200 f \% 20$ Forests $\% 20 t 0 \% 20$ Climate\%20Change.pdf

L'Adaptation des forêts et des populations au changement climatique: un rapport d'évaluation globale (2009). Ce rapport produit par l'Union Internationale des Organisations de Recherche Forestière évalue les corrélations entre les écosystèmes forestiers, les services qu'ils fournissent et le changement climatique. Il évalue également les effets passés et futurs du changement climatique sur les écosystèmes forestiers et les populations qui dépendent de ces écosystèmes ainsi que les options politiques et de gestion en perspective de l'adaptation.

http://www.fao.org/forestry/17675-022721682d6ef9 e51076d5dc46577a9c9.pdf

Rendre les forêts adaptées au changement climatique: une vision globale des effets du changement climatique sur les forêts et les populations et des options d'adaptation (2009). Cette publication est axée sur une évaluation complète des informations scientifiques concernant les effets du changement climatique et la manière par laquelle les forêts et les populations peuvent s'y adapter. Elle fournit également des informations essentielles pour renforcer la capacité d'adaptation des forêts et des populations face au changement climatique. http://www.iufro.org/download/ file/26204/4496/Policy_Brief_ENG_final_pdf/

Les effets du changement climatique sur les forêts européennes et options d'adaptation (2008). Cette étude compile et résume les connaissances sur les effets observés et escomptés du changement climatique sur les forêts en Europe et examine les options d'adaptation des forêts et de la foresterie au changement climatique. https://ec.europa. eu/agriculture/sites/agriculture/files/externalstudies/2008/euro-forests/full_report_en.pdf 
L'Adaptation à la variabilité et au changement climatiques: un manuel d'orientation pour une planification du développement (2007). Ce manuel a été élaboré pour aider les gestionnaires de projets à comprendre comment le changement climatique peut affecter les résultats de leurs projets et à identifier les options d'adaptation à intégrer dans la conception pour rendre les projets plus résilients.

http://pdf.usaid.gov/pdf_docs/PNADJ990.pdf

\section{6) LARESTAURATION DES FORÊTS ET DES PAYSAGES}

Un guide à la méthodologie d'évaluation des opportunités de restauration (ROAM) - Évaluation des opportunités de restauration des paysages forestiers au niveau national ou sous-national (2014). La méthodologie d'évaluation des opportunités de restauration (ROAM) décrite dans ce manuel fournit un cadre flexible et accessible aux pays pour identifier et analyser rapidement le potentiel de restauration des paysages forestiers (FLR) et localiser les zones spécifiques d'opportunités au niveau national ou sous-national. Une application ROAM est généralement entreprise par une petite équipe d'évaluation de base par le biais d'un engagement collaboratif avec d'autres experts et acteurs. Une évaluation au niveau national nécessite généralement, de la part de l'équipe d'évaluation, 15 à 30 jours de travail étalé sur une période de deux à trois mois. https://portals.iucn.org/ library/sites/library/files/documents/2014-030.pdf

Le potentiel mondial de restauration des arbres (2019). La restauration des terres forestières à l'échelle mondiale pourrait contribuer à capturer du carbone atmosphérique et à atténuer le changement climatique. Ce rapport utilise des mesures directes du couvert forestier pour générer un modèle de restauration potentielle des forêts dans le monde entier. Des cartes explicites sur le plan spatial montrent l'étendue de la couverture forestière supplémentaire qui pourrait exister en dehors des forêts et des terres agricoles et urbaines existantes. https://science.sciencemag.org/ content/365/6448/76.abstract

Un cadre coûts-bénéfices pour l'analyse des décisions de restauration des paysages forestiers (2015). Ce rapport présente un cadre coûtsbénéfices permettant de recenser les services écosystémiques et les impacts économiques des activités de restauration des paysages forestiers d'une manière qui permette de structurer les résultats afin de renseigner plusieurs types de décisions de restauration qui peuvent aider les décideurs à comprendre les compromis des différents scénarios de restauration. Les résultats peuvent être utilisés pour fixer le prix du paiement des services écosystémiques, identifier les sources de financement de la restauration, identifier les pistes à faible coût/forte rentabilité concernant la séquestration du carbone et identifier les paysages prioritaires pour une restauration basée sur l'analyse du retour sur investissement. https://portals.iucn.org/library/sites/library/files/ documents/2015-018.pdf

L'agroforesterie au service de la restauration des paysages (2017). Cette publication de la FAO examine le potentiel des systèmes agroforestiers à restaurer la productivité des terres, à conserver la biodiversité, à accroître la résilience des agroécosystèmes, à réduire la pauvreté et à contribuer à la sécurité alimentaire et à la nutrition notamment dans le contexte du changement climatique. http://www.fao.org/3/b-i7374e.pdf 


\title{
Annexe 4. Sous-étapes et méthodologies pour prioriser les options d'adaptation
}

\author{
Adapté de Brugère et De Young, (à paraître)
}

\section{Processus de priorisation}

Le processus de priorisation des options d'adaptation qui ont été identifiées peut prendre plusieurs formes. Le processus de priorisation suggéré ici commence par une évaluation générale de toutes les options d'adaptation pré-identifiées. Ensuite, il passe à une présélection des «top x» options d'adaptation les plus appropriées en fonction de leur adéquation, de leur impact, des avantages pour les moyens d'existence et les écosystèmes, de leur accessibilité et de la capacité à les mettre en œuvre. Il se termine par une analyse plus fine de chaque option présélectionnée sur la base de critères plus spécifiques afin de déterminer ce qui doit être choisi et retenu.

\section{Sous-étape 1: Évaluation/champ d'application générale de toutes les options d'adaptation possibles préalablement identifiées.}

Plusieurs méthodes peuvent être envisagées. Nous présentons ici les SWOT (Forces, Faiblesses, Opportunités, Menaces), les SCORE (Forces, Défis, Options, Réponses, Efficacité) et les méthodes de structuration des problèmes. La méthode à choisir - individuellement ou en combinaison, le cas échéant - est toutefois laissée à l'appréciation des utilisateurs de ces directives supplémentaires. Cela dépend également du fait que le processus global du PNA ait adopté ou non une méthode et que celle-ci soit identifiée.

Une analyse SwOT (Forces, Faiblesses, Opportunités, Menaces) pourrait être effectuée pour identifier pour chaque option d'adaptation, ses forces, faiblesses, opportunités ainsi que les menaces pour les activités encours concernées. Alors que les forces et les opportunités concernent l'option d'adaptation elle-même, les opportunités et les menaces sont liées aux facteurs positifs et négatifs qui affectent la faisabilité et la mise en œuvre de l'option d'adaptation. Si elle est choisie, il est important que cette analyse soit aussi autant spécifique que possible, par exemple qu'elle soit subdivisée en fonction de l'échelle de chaque option d'adaptation et de son contexte spatial et temporel. Cet exercice est basé sur des informations et des expériences connues et non sur la production de nouvelles informations. Ce qui est inconnu (manque d'information/de connaissances) doit être clairement marqué comme une faiblesse ou une menace, selon les circonstances.

Une méthode apparentée est le SCORE (Forces, Défis, Options, Réponses et Efficacité). Comme dans la méthode SWOT, les influences internes et externes sont prises en compte. Cependant, SCORE va plus loin en ajoutant une dimension à la fois temporelle et stratégique à l'analyse grâce à une prise en considération du potentiel actuel (forces et défis) et la durabilité future (options, réponses et critères d'efficacité) d'une option d'adaptation. En outre, il est plus adapté qu'une analyse SWOT pour identifier les manquements potentiels en matière de ressources là où des investissements sont nécessaires. Ici aussi, chaque option d'adaptation potentielle devrait être examinée à tour de rôle selon les cinq critères $\mathrm{SCORE}^{10}$.

\section{- Forces/compétences/soutien:}

- Qu'est-ce que nous pensons être les forces de cette option?

- Quelles sont les compétences et le soutien (y compris les ressources) dont nous disposons pour la mettre en œuvre? Lesquels pouvonsnous demander aux autres?

\section{- Défis / contraintes / compétences nécessaires:}

- Quels sont les problèmes auxquels nous sommes confrontés dans la mise en œuvre de l'adaptation elle-même et par rapport à d'autres facteurs externes?

- Qu'est-ce qui est susceptible de freiner cette option, d'empêcher tout changement nécessaire?

10 Adapté de Moore et al., 2014, et http://weblog.tetradian.com/2013/06/29/checking-the-score/. 
- Comment allons-nous résoudre ou contourner ces contraintes?

- Quelles sont les nouvelles compétences et le soutien dont nous aurions besoin? Que faudrait-il pour les acquérir

\section{- Opportunités et risques:}

- Quels sont les risques susceptibles d'apparaître et les opportunités susceptibles d'être générées et qui devraient être saisis par rapport à la mise en œuvre de cette option d'adaptation?

- Compte tenu des forces et des défis identifiés, cette option d'adaptation est-elle adaptée dans le temps?

\section{- Réponses / retours / récompenses:}

- Quelles sont les conséquences probables ou émergentes de l'action ou de l'inaction?

- Quelles réponses à l'option d'adaptation pouvons-nous attendre des acteurs (directes et indirectes, à différentes échelles)?

- Quels sont les avantages attendus (mesurés par rapport aux risques)?

\section{- Efficacité / impact:}

- Comment pouvons-nous optimiser l'utilisation des ressources pour mettre en œuvre cette option d'adaptation?

- Quels avantages en tirera-t-on? Pour qui, quoi?

- Dans quelle mesure les effets positifs serontils prévisibles et fiables à long terme?

- Créera-t-elle des synergies positives avec d'autres formes d'adaptation et de développement et avec des systèmes socioécologiques plus larges? Y a-t-il des impacts négatifs potentiels à grande échelle que nous devrions connaître?

Les critères de la méthode SCORE sont généralement notés, ce qui permet aux options les plus appropriées de se démarquer grâce aux opportunités qu'elles présentent en termes de forces et d'opportunités de surmonter les défis. Ce système de notation est détaillé au point (ii) Sélection des actions appropriées dans la section suivante. La Barbade a utilisé avec succès la méthode SCORE dans sa notation des options pour rendre son économie plus écologique (Moore et al., 2014).

Pour chaque option d'adaptation présélectionnée, les méthodes SWOT et SCORE doivent mettre en évidence, quoique à des proportions légèrement différentes, les éléments suivants:
- Quelles sont les connaissances, les compétences (individuelles et institutionnelles) et les insuffisances et contraintes financières?

- Quelles sont les autres acteurs avec lesquels il faut s'engager?

- Quels sont les obstacles potentiels à la planification, à la conception et à la mise en œuvre des mesures d'adaptation?

- Quelles sont les options d'adaptation qui semblent les plus réalisables, maintenant et à l'avenir?

Un autre type de méthode permettant d'amorcer la priorisation des options d'adaptation est celui des méthodes de structuration des problèmes (MSP). L'adaptation au changement climatique a été décrite comme un problème non structuré ou «méchant» (Incropera, 2015) parce qu'elle implique de multiples acteurs et perspectives conciliant des intérêts incommensurables et/ou conflictuels et représentant des incertitudes clés, importantes et intangibles; ce qui soulève des questions complexes de gouvernance (Termeer et al., 2013). Les méthodes de structuration des problèmes peuvent être plus ou moins formelles allant du brainstorming, de la cartographie cognitive et de l'analyse multicritères à des approches plus avancées telles que «l'approche des choix stratégiques» et l'élaboration et «l'analyse d'options stratégiques» conçues spécifiquement pour la structuration des problèmes. Menées dans le cadre d'un groupe/atelier, ces méthodes permettent l'exploration conjointe d'une situation ou d'un problème et des pistes potentielles pour l'améliorer ou le résoudre. Les méthodes PSM (Yearworth, 2015):

1. ne sont pas mathématiques mais structurés et rigoureux et reposent sur une modélisation qualitative et schématique;

2. permettent d'exprimer/explorer/de réconcilier une série de points de vue distincts et permettent d'atteindre des objectifs multiples et contradictoires;

3. encouragent la participation active des acteurs au processus de modélisation qualitative/ visuelle à travers des ateliers encadrés et une accessibilité des connaissances;

4. peuvent faciliter la négociation d'un agenda commun et l'appropriation des implications de l'action;

5. une incertitude significative est à prévoir et à tolérer; 
6. fonctionnent de manière itérative;

7. visent l'exploration, l'apprentissage et l'engagement des acteurs.

Ces méthodes constituent une forme de stratégie collaborative fondée sur les interactions entre acteurs à travers laquelle les options d'adaptation à des menaces spécifiques du changement climatique peuvent être discutées pour évaluation et priorisation tout en tenant compte de l'incertitude inhérente et des multiples avantages et contraintes de chaque option. Bien que des perspectives divergentes sur les problèmes eux-mêmes et les facteurs contextuels externes puissent mettre à rude épreuve la collaboration (Head et al., 2016), ces méthodes peuvent fonctionner à plusieurs échelles et contribuer à «recadrer les problèmes et à établir des liens dans le cadre d'accords régionaux à plusieurs niveaux ainsi qu'à relier la science, la politique et les milieux communautaires en matière de politique, de planification et de pratique» (ibid, p. 91). Ce qui est nécessaire pour une adaptation climatique efficace et durable.

Il existe un nombre important d'applications de PSM dans la planification forestière participative (Khadka et al., 2013). Les techniques visuelles et orales sur lesquelles repose le PSM (par exemple les cartes) qui sont alignées sur des techniques participatives et avec les principes de la participation, les rendent particulièrement adaptées aux contextes de faible alphabétisation ou à l'engagement avec des groupes d'acteurs de statuts et de capacités variés. Lors de l'utilisation de la PSM, il convient de contrôler les influences de pouvoir au sein des acteurs concernés car elles peuvent saper les efforts de collaboration en particulier si les options d'adaptation envisagées dépassent les échelles institutionnelles et géographiques (Yearworth, 2015).

En plus d'être utile lors de l'examen des multiples facettes des options d'adaptation potentielles en vue de leur priorisation, la PSM peut également être utile pour l'examen, la vérification et la validation des caractéristiques des effets climatiques relevés lors des étapes initiales de l'élément B avec les acteurs impliqués dans la priorisation des options et leur choix final pour l'intégration dans le PNA. Par exemple, certaines des questions à traiter sont les suivantes: qui sont les acteurs et les parties concernées et leurs valeurs, les niveaux d'incertitude, les questions clés, les influences des environnements externes, les contraintes et les possibilités d'action ainsi que les objectifs d'adaptation. Ce processus de validation peut baliser le chemin vers la mise en œuvre d'analyses décisionnelles multicritères (MCSA) (Belton et Stewart, 2010) qui peuvent être utilisées pour aider à l'évaluation des options d'adaptation les plus appropriées parmi la liste des priorités (voir point (iii) analyse «plus poussée»).

\section{Sous-étape 2: Mesurer et noter les options d'adaptation les plus appropriées.}

Le processus d'interprétation et de pondération de chaque option doit être dirigé par des acteurs pertinents et être liés aux objectifs primordiaux de l'adaptation à réaliser dans chaque système considéré. Un consensus des acteurs est nécessaire pour convenir de ce qui constitue les options les plus appropriées pour chaque système (PNUE, 2012). Les options ayant obtenu les meilleurs scores doivent passer à l'étape suivante de la priorisation. Les acteurs prenant part à l'exercice de priorisation doivent discuter et s'accorder sur le seuil à ne pas dépasser entre les options qui arrivent à l'étape (iii) et celles qui n'y arrivent pas.

Comme indiqué à l'étape précédente, il est indispensable que les principaux acteurs - les hommes et femmes locaux qui dépendent directement et indirectement des forêts et des arbres - soient largement consultés au cours de ce processus car ils sont en première ligne du changement climatique et seront les premiers concernés et affectés par les options d'adaptation choisies.

Toutes les options d'adaptation potentielles, planifiées et autonomes, auront été élucidées à l'étape précédente. L'objectif ici d'avoir une liste évaluée et classifiée du potentiel de chaque option d'adaptation en fonction des pondérations ou des notes attribuées pour réduire la vulnérabilité au changement climatique avant de procéder à une analyse plus fine de la faisabilité économique de chaque option (voir la sous-étape 3).

Nous proposons ici deux méthodes légèrement différentes visant à aider à la priorisation et à l'identification des options d'adaptation les plus prometteuses: l'une basée sur la pondération des critères importants et la notation des options d'adaptation en fonction de ceux-ci; l'autre basée sur la notation uniquement. Elles constituent une suite de la méthode SCORE décrite précédemment. Compte tenu de l'importance de la participation des acteurs à ce processus, les deux méthodes devraient être exécutées au cours d'un atelier. 


\section{La pondération et la notation}

Mesurer l'importance commence par le choix de critères (ou indicateurs) et le crédit à accorder à l'évaluation des options d'adaptation préidentifiées. Cette évaluation doit être effectuée par les acteurs participant à l'exercice. Elle doit en outre être spécifique au contexte (voir l'encadré 16 pour un certain nombre de ces critères). Bien qu'ils soient classés en deux grands groupes, «impact» et «viabilité», les critères choisis doivent être harmonisés autant que possible avec les objectifs d'adaptation choisis et préalablement déterminés.

Une fois les critères choisis, il est important de se rappeler que la sélection faite doit présenter les caractéristiques suivantes (USAID, 2013; DCLG, 2009):

- La complétude: Tous les critères importants ont-ils été inclus?

- La redondance: Certains critères sont-ils non nécessaires ou redondants?

- L'opérationnalité: Les critères sont-ils mesurables ou définis?

- L'Indépendance mutuelle: La performance d'une option par rapport à un critère est-elle indépendante de la performance de la même option par rapport à un second critère?
- Le double comptage: Deux critères comptent-ils pour la même question?

- La taille: Y a-t-il trop de critères?

- Les effets dans le temps: Les effets différenciés dans le temps sont-ils correctement pris en compte dans l'ensemble des critères?

Une fois que les critères de caractérisation des mesures d'adaptation ont été approuvés, il est possible de donner une note en demandant aux acteurs d'attribuer individuellement un nombre fixe de points (par exemple sur 100) à tous les critères choisis afin de refléter leur importance en termes généraux (et non par rapport à chaque option d'adaptation potentielle). Les moyennes des points attribués constituent le poids, c'est-à-dire l'importance à accorder. Les écarts types doivent également être pris en compte et faire l'objet d'un débat ouvert afin de garantir un consensus général entre les acteurs sur les pondérations finalement attribuées. Une autre alternative est que l'attribution du nombre de points peut être effectuée conjointement par tous les acteurs au cours d'une séance plénière encadrée.

\section{ENGADRÉ 16}

\section{Exemples de critères génériques importants pour évaluer les options d'adaptation possibles}

\section{IMPACT}

- Timing/urgence de l'action d'adaptation [quelles sont les actions requises: face aux conséquences des retards sur la vulnérabilité, pour prendre en charge les effets à court, moyen et/ou long terme et les éventuelles mises en œuvre séquentielles].

- Cobénéfices/équité générés par l'action d'adaptation, par exemple en termes de réduction des émissions, d'augmentation de la productivité et des revenus [notamment le bénéficiaire et le perdant en tenant pleinement compte des considérations d'équité des genres, sociale et intergénérationnelle].

- Effets secondaires, positifs ou négatifs [impacts sur la fourniture de services écosystémiques notamment les processus biophysiques, les moyens d'existence; les effets secondaires et intersectoriels, les compromis].

- Contribution et synergies supplémentaires [[possibilité de prendre en charge des questions autres que la résilience climatique. Par exemple, la réduction de la pauvreté, le bien-être, la pertinence stratégique par rapport à des objectifs de développement plus larges et durables]. 


\section{VIABILITÉ}

- Différents scénarios climatiques, changements rapide et lent, prévention des dommages irréversibles.

- Flexibilité et/ou robustesse de l'action d'adaptation [possibilité pour l'action d'adaptation d'évoluer/de rester fixe dans le temps, si le système change après l'adaptation, si des effets non prévus initialement se produisent et/ou si des circonstances externes affectent la mise en œuvre].

- Acceptation sociale et politique/légitimité

- Obstacles et capacité de mise en œuvre [dépendance à l'égard d'autres secteurs pour adapter et améliorer en même temps (par exemple, la gestion de l'eau, le tourisme, la législation)].

NB: les dimensions économiques sont traitées plus en détail dans la sous-étape 3.

Source: élaboré à partir de LEG, 2012; Hahn et Fröde, 2011; Banque mondiale 2010a, 2009.

Afin d'évaluer chaque mesure d'adaptation par rapport à chaque critère pondéré, les acteurs concernés reçoivent ensuite une matrice et sont invités à donner une valeur comprise entre 1 («pas du tout») à 10 (《complètement») qui reflète à quel point ils estiment que chaque critère est effectivement pris en charge par chaque option d'adaptation. Par exemple, «l'option d'adaptation 1 permettra-t-elle de répondre en temps utile à la menace identifiée $\mathrm{x}$ ?», 《l'option d'adaptation 1 aura-t-elle la capacité de générer des cobénéfices»? Les scores (dont la moyenne est calculée si chaque participant a noté individuellement ou est pris comme tel si la notation donnée en séance plénière) de chaque option d'adaptation par critère sont ensuite pondérés par rapport aux critères de pondération précédemment calculés. Si nécessaire, les critères globaux «d'impact» et «de viabilité» peuvent se voir attribuer une pondération globale de 50 pour cent chacun. Toutefois, cela peut faire l'objet de discussions selon les perceptions des acteurs et du nombre de catégories. Les résultats sont ensuite multipliés par 100 pour ramener les notes pondérées à une valeur maximale de 100. Les options d'adaptation peuvent alors être classées par ordre décroissant de priorité.

Un certain nombre de pays d'Amérique latine ont utilisé cette approche pour décider de leurs stratégies de réponse au changement climatique dans le secteur agricole (Banque mondiale, 2009).

\section{La notation (suite de la méthode SCORE)}

Si la méthode SCORE a été utilisée auparavant, chaque option d'adaptation évaluée peut maintenant être notée. Toutefois, la notation peut également être effectuée de manière indépendante, par exemple pour évaluer globalement les options d'adaptation en termes de leur réalisabilité. Cette méthode permet de mesurer chaque option en fonction de l'«écart de performance» qu'elle représente (c'est-à-dire ce qu'il est nécessaire de combler pour qu'une action d'adaptation réalise pleinement ses objectifs) mais aussi en fonction de l'importance de son efficacité combinée et de son potentiel à générer et à saisir des opportunités. Dans ce cas, l'avis d'un expert peut être utilisé comme alternative à la consultation des acteurs. La Barbade l'a utilisée avec succès pour définir les options permettant de rendre son économie verte. (Moore et al., 2014).

Chacun des défis identifiés lors de l'exercice SCORE se voient attribuer une note sur une échelle de 1 point (très faible) à 10 points (très élevé) en fonction de leur fréquence de survenance ou de leur probabilité d'impact, en veillant à ce qu'ils couvrent non seulement les limitations de ressources mais aussi ce qui doit changer (par exemple, les capacités, les services). Comme indiqué précédemment, un certain nombre de critères supplémentaires peuvent être utilisés. La combinaison (c'est-à-dire la duplication) des deux (ou plus si plus de critères ont été utilisés) scores donne une valeur globale du niveau de gravité 
du défi. Par conséquent, un défi avec une valeur proche de 1 signifie qu'il est perçu comme n'étant pas grave tandis qu'un défi avec une note proche de $10^{11}$ est perçu comme extrêmement grave.

Le deuxième élément de l'analyse des écarts consiste à attribuer une note au niveau actuel de l'option d'adaptation, c'est-à-dire ses forces pour relever chaque défi à partir de 1 indiquant qu'elle ne dispose pas pour le moment du nécessaire, jusqu'à 10 indiquant qu'elle en dispose totalement. En retirant les scores des défis des scores des forces pour chaque défi, on a une idée du potentiel de l'action d'adaptation à surmonter chaque défi énuméré: plus la valeur négative est grande, plus l'amplitude du «déficit de performance» et des efforts/investissements à déployer pour le combler sont importants.

Quant aux défis, chaque opportunité présentée par l'option d'adaptation peut être notée sur une échelle de 1 à 10 points selon sa probabilité de survenance et sa probabilité d'efficacité. Les scores de survenance et d'efficacité sont ensuite multipliées pour obtenir un indicateur d'importance quantifiée (ou d'attractivité) pour chaque opportunité. Ce qui offre la possibilité de les classer (plus le nombre est élevé, sur 100, plus l'importance est grande).

\section{Sous-étape 3: L'Analyse (économique) plus fine et la décision finale sur les options les plus appropriées.}

Cette sous-étape est axée sur la faisabilité économique, sociale et environnementale, c'està-dire les coûts et les bénéfices des options d'adaptation qui sont ressorties comme les «meilleures candidates» des précédentes étapes de priorisation.

L'estimation des coûts et des bénéfices de chaque option est un aspect intégral mais potentiellement compliqué du processus de priorisation. C'est la raison pour laquelle elle est traitée séparément ici. En fait, elle est importante car elle va assurer la visibilité des options d'adaptation dans le PNA global où ces options vont côtoyer celles faites pour d'autres secteurs. Toutefois, il est également important de garder à l'esprit que l'évaluation de tous leurs bénéfices, y compris ceux des autres secteurs, peut être difficile à appréhender pleinement dans les analyses économiques et par conséquent, les options d'adaptation peuvent sembler plus coûteuses (du moins à court terme) comparées à celles des autres secteurs.

Si les étapes de priorisation précédentes (i) et (ii) peuvent être exécutées sur une base consultative, par exemple dans le cadre d'un atelier réunissant les acteurs concernés, cette analyse économique plus fine en tant que telle requiert probablement plus de temps et d'expertise. Cette expertise peut être obtenue soit au sein de la cellule qui supervise le processus de formulation et de mise en œuvre du PNA ou sous-traitée. Bien qu'elle soit susceptible d'être plus adéquatement pilotée par des experts, une consultation des acteurs impliqués dans les étapes précédentes de la priorisation et/ou du processus global sera nécessaire pour recueillir leur avis et leur approbation sur les résultats de cette analyse.

Il existe plusieurs méthodes pour évaluer les coûts des options d'adaptation. Leur choix sera guidé par les objectifs d'adaptation et le nombre d'options d'adaptation potentielles identifiées précédemment ainsi que par les données disponibles.

La figure 6 présente un arbre de décision simplifié qui sert de guide pour le choix de la méthode la plus appropriée.

L'estimation des coûts et des bénéfices de l'adaptation doit tenir compte des questions d'incertitude, d'équité (effets distributifs) et d'évaluation (bases de référence, types d'évaluation, taux d'actualisation et horizons temporels), qui sont particulièrement aiguës dans le contexte d'adaptation au climat. Certaines des méthodes présentées ci-dessous sont plus ou moins adaptées pour traiter ces questions. Par conséquent, elles sont plus ou moins adaptées à l'évaluation finale des options d'adaptation (CCNUCC, 2011b; Banque mondiale, 2010b).

11 Après avoir divisé le score global par 10 pour normaliser les scores et les rendre comparables à ceux des étapes suivantes. 


\section{FIGURE 6}

Arbre de décision (simplifié) des méthodes potentielles pour évaluer les coûts et les bénéfices des options d'adaptation

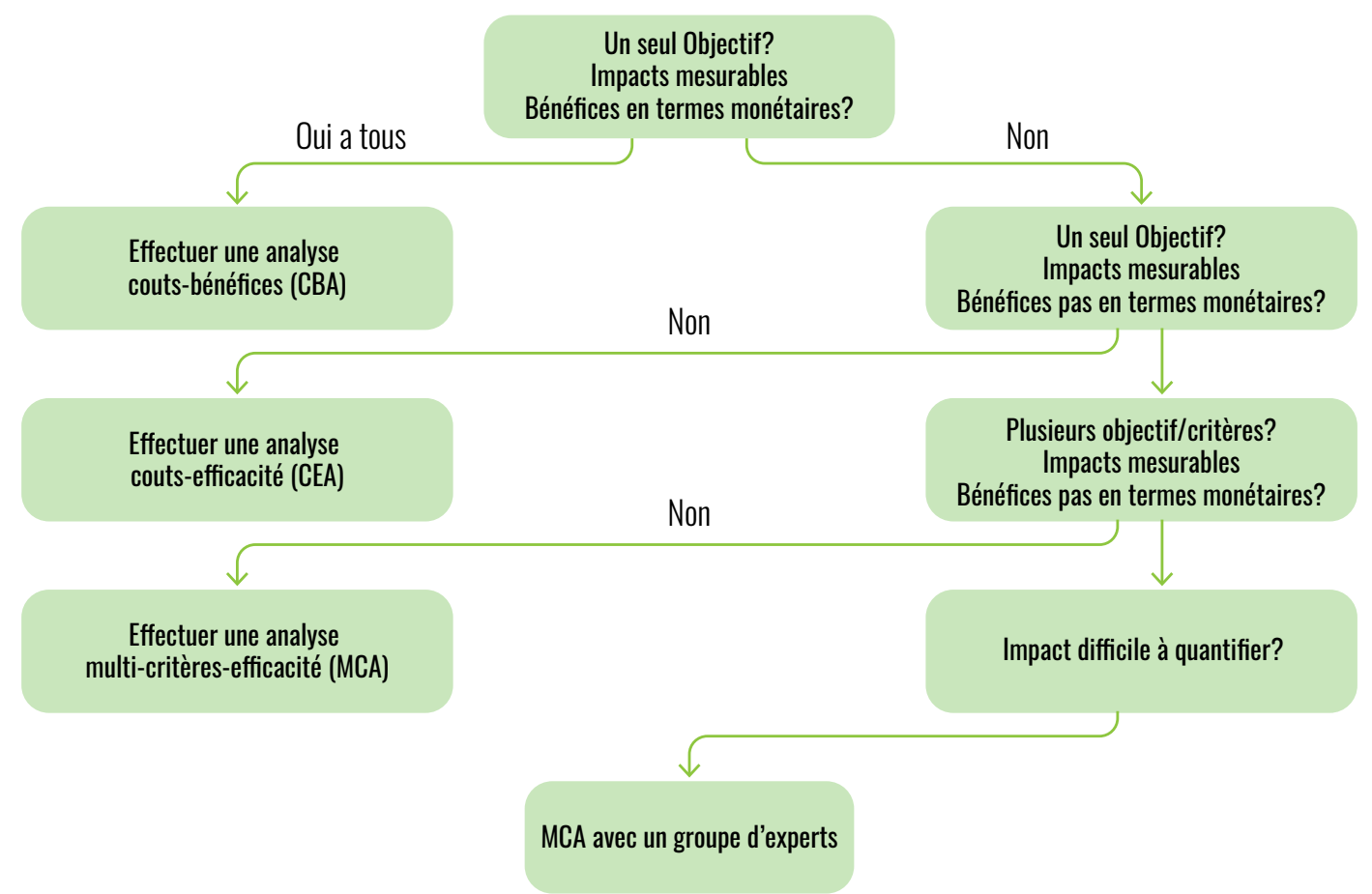

Source: CCNUCC, 2011 .

L'analyse coûts-bénéfices (ACB), l'analyse coûtefficacité (ACE) et l'analyse (décisionnelle) multicritères (AMC ou AMCD) sont mises en évidence dans la figure 6. Ce sont là les trois méthodes les plus communément utilisées pour appuyer l'évaluation économique des options d'adaptation dans le secteur agricole. Le tableau 3 résume leurs objectifs, leurs points positifs et négatifs ainsi que leurs besoins en données. Il décrit également le processus d'analyse hiérarchique (AHP), une sorte de MCA de plus en plus utilisée dans le contexte d'adaptation au changement climatique. Il s'agit toutefois d'un domaine en constante évolution. D'autres méthodes plus aptes à gérer l'incertitude sont maintenant disponibles: l'analyse par les options réelles, la prise de décision robuste, l'analyse de portefeuille, la gestion itérative du risque (adaptation). Un aperçu de toutes ces méthodes est disponible dans Watkiss et Hunt (2013 - en libre accès). 
TABLEAU 3

\section{Résumé des caractéristiques des principales méthodes d'aide à la décision (économique) pour le choix des mesures d'adaptation au climat.}

\begin{tabular}{|c|c|c|c|c|c|}
\hline MÉTHODE & $\begin{array}{l}\text { OBJECTIF } \\
\text { D'UTILISATION }\end{array}$ & FORCES & LIMITES & DONNÉES REQUISES & $\begin{array}{l}\text { RESSOURCES ET } \\
\text { EXPERTISE }\end{array}$ \\
\hline $\begin{array}{l}\text { Analyse Coûts- } \\
\text { Bénéfices (ACB) }\end{array}$ & $\begin{array}{l}\text { Évaluer tous les } \\
\text { coûts et bénéfices } \\
\text { de toutes les } \\
\text { options et estime } \\
\text { les bénéfices/ } \\
\text { coûts nets des } \\
\text { interventions } \\
\text { individuelles } \\
\text { en termes } \\
\text { monétaires. } \\
\text { Mesure du } \\
\text { bénéfice: } \\
\text { Économique } \\
\text { (monétaire). }\end{array}$ & $\begin{array}{l}\text {-Fournit une } \\
\text { analyse directe } \\
\text { des bénéfices } \\
\text { économiques, de } \\
\text { la justification } \\
\text { des actions et } \\
\text { des solutions } \\
\text { optimales. } \\
\text { - Reconnu et } \\
\text { largement } \\
\text { appliqué. }\end{array}$ & $\begin{array}{l}\text {-Difficulté dans } \\
\text { l'évaluation } \\
\text { monétaire pour } \\
\text { les secteurs non } \\
\text { marchands et } \\
\text { les options non } \\
\text { techniques. } \\
\text { - L'incertitude est } \\
\text { généralement } \\
\text { limitée aux } \\
\text { risques } \\
\text { probabilistes. }\end{array}$ & $\begin{array}{l}\text {-Les données } \\
\text { des scénarios } \\
\text { individuels } \\
\text { et du modèle } \\
\text { climatique. } \\
\text { - Les coûts } \\
\text { de base des } \\
\text { dommages liés } \\
\text { à l'évaluation } \\
\text { d'impact du } \\
\text { scénario. } \\
\text { Efficacité de } \\
\text { l'adaptation } \\
\text { quantitative. }\end{array}$ & Moyen \\
\hline $\begin{array}{l}\text { Analyse Coût- } \\
\text { Efficacité (ACE) }\end{array}$ & $\begin{array}{l}\text { Comparer } \\
\text { les options } \\
\text { alternatives } \\
\text { pour obtenir } \\
\text { des résultats } \\
\text { (ou objectifs) } \\
\text { similaires en } \\
\text { termes de coûts } \\
\text { comparés à } \\
\text { l'efficacité. } \\
\text { Mesure du } \\
\text { bénéfice: } \\
\text { Quantitatif (mais } \\
\text { pas économique). }\end{array}$ & $\begin{array}{l}\text { Les bénéfices } \\
\text { exprimés } \\
\text { en termes } \\
\text { physiques (et } \\
\text { non monétaires) } \\
\text { donc applicables } \\
\text { aux secteurs non } \\
\text { marchands. } \\
\text { - Elle est } \\
\text { relativement } \\
\text { facile à } \\
\text { appliquer avec } \\
\text { un classement } \\
\text { et des résultats } \\
\text { facilement } \\
\text { compréhensibles. } \\
\text { - L'utilisation des } \\
\text { courbes de coûts } \\
\text { permet d'évaluer } \\
\text { les objectifs } \\
\text { politiques avec } \\
\text { une optimisation } \\
\text { au moindre coût. } \\
\text { - Fréquemment } \\
\text { utilisé pour } \\
\text { l'atténuation, } \\
\text { donc largement } \\
\text { reconnu et ayant } \\
\text { une résonance } \\
\text { auprès des } \\
\text { décideurs. }\end{array}$ & $\begin{array}{l}\text {-Les bénéfices } \\
\text { peuvent être } \\
\text { difficiles à identi- } \\
\text { fier et une mesure } \\
\text { unique ne permet } \\
\text { pas de cerner } \\
\text { tous les coûts et } \\
\text { bénéfices. Elle est } \\
\text { moins applicable } \\
\text { aux questions } \\
\text { intersectorielles / } \\
\text { complexes. } \\
\text { - Elle fonctionne } \\
\text { mieux avec les } \\
\text { options tech- } \\
\text { niques et omet } \\
\text { souvent le } \\
\text { renforcement } \\
\text { des capacités et } \\
\text { les mesures non } \\
\text { contraignantes. } \\
\text { - La nature } \\
\text { séquentielle } \\
\text { des courbes de } \\
\text { coûts ne tient } \\
\text { pas compte des } \\
\text { corrélations et du } \\
\text { potentiel des por- } \\
\text { tefeuilles. } \\
\text { - Elle ne se prête } \\
\text { pas à la prise en } \\
\text { compte de l'in- } \\
\text { certitude car elle } \\
\text { fonctionne avec } \\
\text { une orientation } \\
\text { centrale. }\end{array}$ & $\begin{array}{l}\text {-Les résultats } \\
\text { des scénarios } \\
\text { et du modèle } \\
\text { climatique et } \\
\text { souvent les coûts } \\
\text { de base des } \\
\text { dommages. } \\
\text { - L'efficacité } \\
\text { comme moyen } \\
\text { de réduction des } \\
\text { impacts (unité / } \\
\text { total). }\end{array}$ & Moyen \\
\hline
\end{tabular}




\begin{tabular}{|c|c|c|c|c|c|}
\hline MÉTHODE & $\begin{array}{l}\text { OBJECTIF } \\
\text { D'UTILISATION }\end{array}$ & FORCES & LIMITES & DONNÉES REQUISES & $\begin{array}{l}\text { RESSOURCES ET } \\
\text { EXPERTISE }\end{array}$ \\
\hline $\begin{array}{l}\text { Analyse } \\
\text { Multicritères } \\
\text { (MCA) / } \\
\text { Analyse } \\
\text { Décisionnelle } \\
\text { Multicritère } \\
\text { (MCDA) }\end{array}$ & $\begin{array}{l}\text { Évaluer et noter } \\
\text { systématiquement } \\
\text { les options } \\
\text { alternatives } \\
\text { par rapport } \\
\text { aux critères de } \\
\text { décision choisis. } \\
\text { Mesure du } \\
\text { bénéfice: } \\
\text { Qualitatif, } \\
\text { quantitatif ou } \\
\text { économique. }\end{array}$ & $\begin{array}{l}\text { - Combine } \\
\text { des données } \\
\text { quantitatives et } \\
\text { qualitatives ainsi } \\
\text { que des unités } \\
\text { monétaires et } \\
\text { non monétaires } \\
\text { qui, sont ainsi } \\
\text { applicables } \\
\text { lorsque la } \\
\text { quantification } \\
\text { est difficile. } \\
\text { - Elle est } \\
\text { relativement } \\
\text { simple et } \\
\text { transparente et } \\
\text { son coût/délai } \\
\text { est relativement } \\
\text { faible. } \\
\text { - L'avis des } \\
\text { experts peut } \\
\text { être utilisé de } \\
\text { manière très } \\
\text { efficace et } \\
\text { implique les } \\
\text { acteurs. Il peut } \\
\text { donc être fondé } \\
\text { sur l'expertise } \\
\text { locale. }\end{array}$ & $\begin{array}{l}\text { - Les résultats } \\
\text { doivent faire } \\
\text { l'objet d'une } \\
\text { interprétation et } \\
\text { d'une élaboration } \\
\text { plus poussées } \\
\text { dans le cadre } \\
\text { d'études plus } \\
\text { approfondies. } \\
\text { - Les différents } \\
\text { experts peuvent } \\
\text { avoir des opinions } \\
\text { différentes, c'est- } \\
\text { à-dire que la } \\
\text { subjectivité peut } \\
\text { entrer en jeu. } \\
\text { - Les acteurs } \\
\text { peuvent manquer } \\
\text { de connaissances } \\
\text { et passer à } \\
\text { côté d'options } \\
\text { importantes. } \\
\text { - L'analyse de } \\
\text { l'incertitude } \\
\text { est souvent } \\
\text { qualitative et } \\
\text { subjective. }\end{array}$ & $\begin{array}{l}\text { - Les informations } \\
\text { qualitatives ou } \\
\text { quantitatives sur } \\
\text { le changement } \\
\text { climatique. } \\
\text { - L'efficacité à } \\
\text { travers l'apport } \\
\text { des experts ou la } \\
\text { consultation des } \\
\text { acteurs. }\end{array}$ & Faible à Moyen \\
\hline $\begin{array}{l}\text { Processus } \\
\text { d'Analyse } \\
\text { Hiérarchique } \\
\text { (AHP) }\end{array}$ & $\begin{array}{l}\text { Déterminer des } \\
\text { échelles de prio- } \\
\text { rité à partir de } \\
\text { comparaisons par } \\
\text { paires en utili- } \\
\text { sant les avis des } \\
\text { experts. } \\
\text { Mesure du béné- } \\
\text { fice: } \\
\text { Qualitative, } \\
\text { quantitative ou } \\
\text { économique. }\end{array}$ & $\begin{array}{l}\text { - Elle peut être } \\
\text { appliquée là où } \\
\text { les éléments } \\
\text { sont difficiles } \\
\text { à quantifier } \\
\text { ou ne sont pas } \\
\text { directement } \\
\text { comparables. } \\
\text { - Une méthode } \\
\text { relativement } \\
\text { simple qui établit } \\
\text { des classements } \\
\text { simples et faciles } \\
\text { à communiquer. } \\
\text { - Elle ne } \\
\text { nécessite pas } \\
\text { d'informations } \\
\text { sur les bénéfices } \\
\text { économiques. } \\
\text { Elle a donc une } \\
\text { large applicabilité. } \\
\text { - Elle peut } \\
\text { s'adapter à } \\
\text { plusieurs } \\
\text { disciplines, } \\
\text { opinions et } \\
\text { groupes de } \\
\text { personnes qui, } \\
\text { d’habitude, } \\
\text { n'interagissent } \\
\text { pas. }\end{array}$ & $\begin{array}{l}\text { - Les résultats } \\
\text { changent au } \\
\text { fur et à mesure } \\
\text { que de nouve- } \\
\text { lles options sont } \\
\text { envisagées. } \\
\text { - Elle devient } \\
\text { compliquée si } \\
\text { de nombreux } \\
\text { critères et options } \\
\text { sont pris en } \\
\text { compte. } \\
\text { - Une échelle } \\
\text { subjective peut } \\
\text { conduire à des } \\
\text { erreurs. } \\
\text { - Le renforcement } \\
\text { des capacités } \\
\text { transdisciplinai- } \\
\text { res peut être sapé } \\
\text { aux dépens de } \\
\text { l'opportunité. } \\
\text { - Les logiciels } \\
\text { peuvent } \\
\text { dissimuler } \\
\text { des jugements } \\
\text { de valeur } \\
\text { contradictoires. }\end{array}$ & $\begin{array}{l}\text { - Les informations } \\
\text { qualitatives ou } \\
\text { quantitatives sur } \\
\text { le changement } \\
\text { climatique. } \\
\text { - L'efficacité à } \\
\text { travers l'apport } \\
\text { des experts ou la } \\
\text { consultation des } \\
\text { acteurs. }\end{array}$ & Faible à Moyen \\
\hline
\end{tabular}


En raison des contraintes liées à l'évaluation économique des bénéfices des forêts, l'analyse (décisionnelle) multicritères (AMC ou AMCD) est probablement la méthode la plus appropriée car elle peut aider à surmonter les problèmes de quantification et d'évaluation en termes monétaires. Lorsqu'elle est appliquée à ce stade du processus, une AMC assurerait le suivi de la notation et de la pondération des options effectuées antérieurement (ces étapes préliminaires sont en réalité la première étape d'une AMC), en utilisant les critères énumérés dans l'encadré 16 et les coûts et bénéfices estimés nets ou supplémentaires - associés à chaque option d'adaptation qui, peuvent ou non être exprimés en termes monétaires.

Le MCA consiste à noter les performances de chaque option d'adaptation par rapport à chacun des critères sélectionnés, à normaliser les scores obtenus pour les différents critères et à attribuer un poids à chaque critère pour refléter les priorités de manière à inclure à la fois des critères économiques et non économiques dans le processus de priorisation. Naturellement, il est important d'être très clair sur les résultats escomptés de chaque option d'adaptation ainsi que leur adéquation avec les objectifs d'adaptation fixés.

La nature mixte quantitative-qualitative de l'AMC la rend plus adaptée aux interactions entre les acteurs. C'est le cas, par exemple, dans un atelier au cours duquel la pondération et la notation sont effectuées. Ensuite suivent d'autres méthodes telles que l'ACB. Un autre avantage de l'AMC est sa capacité à faire des analyses de sensibilité avec différents scores ou pondérations: Que se passerait-il si...? Comment se ferait alors la comparaison entre les options? L'ajustement itératif des scores ou des pondérations donne la possibilité d'examiner «l'éventail des possibilités» et donc de tenir compte plus explicitement des différents degrés d'incertitude dans le processus décisionnel concernant le choix final des options d'adaptation.

En fait, l'AMC a été la méthode de choix des pays les moins avancés (PMA) pour le classement des options d'adaptation dans la préparation de leurs PANA (CCNUCC, 2011b). C'est le cas, par exemple, du Rwanda (2006) et de l'Éthiopie (2007).

De Bruin et al. (2009) ont piloté aux Pays-Bas l'utilisation du MCA dans l'évaluation d'une série de potentielles mesures d'adaptation au climat.

\section{L'Organisation et la présentation des informations}

Les résultats du processus de priorisation doivent être présentés sous une forme facilement compréhensible et communicable. Ils peuvent, par exemple, être présentes sous forme de tableau avec un aperçu du classement des options et d'autres informations connexes.

\section{Les tableaux synthétiques}

Les tableaux synthétiques peuvent prendre plusieurs formes. En définitive, ils refléteront les caractéristiques (ou critères) qui ont été mises en avant ou qui ont été jugées essentielles au cours du processus de priorisation. Par exemple, le tableau 4 met l'accent sur les coûts et bénéfices estimés (ceux-ci ne doivent pas nécessairement être monétisés dans le tableau synthétique mais peuvent être considérés comme ayant une valeur élevée, moyenne ou faible. Une option alternative serait de mettre l'accent sur les acteurs responsables de la mise en œuvre et sur les différentes périodes d'action. Il peut également illustrer le nombre important de mesures d'adaptation qui ont été envisagées et le caractère «onéreux» de chaque mesure (Garrett et al., 2015). 


\section{TABLEAU 4}

\section{Un exemple de tableau synthétique des mesures d'adaptation prioritaires selon leurs bénéfices et leurs coûts}

\begin{tabular}{|c|c|c|c|c|c|c|c|c|c|c|c|c|c|c|}
\hline \multirow{3}{*}{$\begin{array}{l}\text { LISTE DES } \\
\text { ADAPTATIONS } \\
\text { (PAR ORDRE } \\
\text { DÉCROISSAN) }\end{array}$} & \multicolumn{8}{|c|}{ BÉNÉFICES } & \multicolumn{4}{|c|}{ COÛTS } & \multirow{2}{*}{\multicolumn{2}{|c|}{$\begin{array}{l}\text { CAPACITÉ DE } \\
\text { MISE EN EUUVEE } \\
* * *\end{array}$}} \\
\hline & \multicolumn{2}{|c|}{$\begin{array}{l}\text { Macro- } \\
\text { économiques }\end{array}$} & \multicolumn{2}{|c|}{$\begin{array}{l}\text { Moyens } \\
\text { d'existence } \\
\text { micro- } \\
\text { économiques }\end{array}$} & \multicolumn{2}{|c|}{ Ecosystème } & \multicolumn{2}{|c|}{$\begin{array}{l}\text { Autre } \\
\text { non- } \\
\text { marchand }\end{array}$} & \multicolumn{2}{|c|}{$\begin{array}{l}\text { Coûts du } \\
\text { capital* }\end{array}$} & \multicolumn{2}{|c|}{$\begin{array}{l}\text { Coûts de } \\
\text { maintenance } \\
* *\end{array}$} & & \\
\hline & CT & MT & CT & MT & CT & MT & CT & MT & CT & MT & CT & $\mathrm{v}$ & CT & MT \\
\hline A & & & & & & & & & & & & & & \\
\hline B & & & & & & & & & & & & & & \\
\hline C & & & & & & & & & & & & & & \\
\hline & & & & & & & & & & & & & & \\
\hline & & & & & & & & & & & & & & \\
\hline & & & & & & & & & & & & & & \\
\hline
\end{tabular}

Source: élaboré à partir du PNUE, 2012

ST: court terme, MT: moyen terme. ${ }^{*}=$ coûts d'investissement, $* *=$ coûts de fonctionnement. ***pourrait être divisé davantage selon qu'il soit individuel (agriculteurs, pêcheurs) ou institutionnel (autorités locales, gouvernement national, services décentralisés etc.) ou selon les coûts (par exemple la formation) qui devront être engagés pour renforcer les capacités à des niveaux requis.

La notation: 0 = néant, 1 = bas, 2 = moyen, 3 = élevé. Pour les coûts d'investissement et de maintenance, la notation doit être inversée.

Une autre façon possible d'organiser et de synthétiser les informations obtenues jusque-là est celle basée graphiquement sur les critères utilisés dans le processus de priorisation. On peut faire cela tout en soutenant une décision finale pour laquelle les mesures d'adaptation sont mises en avant dans le PNA .

Les options sans regret et à faible regret peuvent être des options « gagnant-gagnant» lorsqu'elles renforcent la capacité d'adaptation (c'est-à-dire qu'elles réduisent la vulnérabilité climatique et exploitent les opportunités positives), tout en contribuant à l'obtention de résultats sociaux, environnementaux ou économiques supplémentaires. L'adaptation à haut niveau de regret implique principalement des décisions de planification à grande échelle (par exemple, le redéploiement d'une population importante) ainsi que des investissements à forte irréversibilité (par exemple, de grands projets d'infrastructure tels que les digues de protection contre le niveau de la mer). Étant donné les conséquences considérables qui sont en jeu dans les décisions de planification à grande échelle et les coûts d'investissement importants ainsi que la longue durée de vie des infrastructures, les incertitudes des projections climatiques futures doivent être examinées avec soin. La différenciation entre l'adaptation sans regret, à faible regret et à fort regret n'est pas universelle, mais dépend des circonstances locales et de l'horizon temporel. Il est important d'être conscient du niveau de regret car les différents niveaux de regret ont des implications différentes dans les domaines de l'information climatique, du calendrier des investissements, de l'horizon de planification, de la conception du projet, des risques du projet et de son évaluation économique (Banque mondiale, 2010a). Cela signifie également que l'incertitude est intégrée dans le classement des options d'adaptation. 


\section{Références}

AC-LEG. 2016. Une contribution aux discussions du Comité d'adaptation et du Groupe d'experts des pays les moins avancés lors de leurs réunions sur leur travail dans le cadre des mandats contenus dans la décision 1/CP.21, paragraphes 41 et 45. Examen documentaire par le Secrétariat. Groupe d'experts des pays les moins avancés du Comité d'adaptation. (également disponible à l'adresse https://unfccc.int/files/adaptation/groups_committees/ adaptation_committee/application/pdf/ac-leg2_mandates.pdf).

Balikoowa, K., Nabanoga, G. et Tumusiime, D.M. 2018. Gender stereotyping: Evidence from gender differentiated household vulnerability to climate change in Eastern Uganda. Cogent Environmental Science, 4(1): 1-15.

Banque mondiale. 2009. Building Response Strategies to Climate Change in Agricultural Systems in Latin America. The Banque mondiale, Washington D.C. (également disponible à l'adresse https://openknowledge.worldbank.org/handle/10986/12473).

Banque mondiale. 2010a. Mainstreaming adaptation to climate change in agriculture and natural resources management projects. Guidance Note 6. Banque mondiale, Washington D.C. (également disponible à l'adresse http://siteresources.worldbank.org/EXTTOOLKIT3/Resources/3646250-1250715327143/GN6.pdf).

Banque mondialek. 2010b. Economic evaluation of climate change adaptation projects. Approaches for the agricultural sector and beyond. Banque mondiale, Washington, D.C. (également disponible à l'adresse https://www.preventionweb.net/files/14726_DevCC1Adaptation1.pdf).

Bee, B.A. 2016. Power, perception and adaptation: exploring gender and social-environmental risk perception in northern Guanajuato, Mexico. Geoforum, 69: 71-80.

Bele, M.Y., Tiani, A.M., Somorin, O.A. et Sonwa, D.J. 2013. Exploring vulnerability and adaptation to climate change of communities in the forest zone of Cameroon. Climatic Change, 119(3-4): 875-889.

Belton, V. et Stewart, T. 2010. Problem structuring and multiple criteria decision analysis. In: M. Ehrgott, J. Rui Figueira and S. Greco (eds.) Trends in Multiple Criteria Decision Analysis. Springer, pp. 209-239.

Bogaert, J., Barima, Y.S.S,. Mongo, L.I.W., Bamba, I., Mama, A., Toyi, M. et Lafortezza, R. 2011. Forest fragmentation: causes, ecological impacts and implications for landscape management. In C. Li, R. Lafortezza \& J. Chen, eds. Landscape ecology in forest management and conservation. Challenges and solutions for global change, $\mathrm{pp}$. 273-296. Beijing, Higher Education Press, and Berlin/Heidelberg, Springer-Verlag.

Borja-Vega, C. et de la Fuente, A. 2013. Municipal vulnerability to climate change and climate related events in Mexico. Washington DC, Banque mondiale.

Brody, A., Demetriades, J. et Esplen, E. 2008. Gender and climate change: mapping the linkages - A scoping study on knowledge and gaps. Brighton, UK, Institute of Development Studies.

CBD. 2014. Addressing Water in National Adaptation Plan. Water Supplement to the UNFCC NAP Technical Guidelines. (également disponible à l'adresse https://www4.unfccc.int/sites/NAPC/Documents/Supplements/GWP_NAP_Water_Supplement_May2019.pdf).

CCFM. 2008. A vision for Canada's forests: 2008 and beyond. Groupe de travail sur le changement climatique du Conseil canadien des ministres des forêts.

CIFOR. 2011. Forests, Trees and Agroforestry: Livelihoods, Landscapes and Governance. CGIAR Research Programme on Forests, Trees and Agroforestry (FTA) Proposal. Bogor, Indonesie: CIFOR.

Climate Change Commission. 2011. The Philippines: National Climate Change Action Plan 2011-2028. Manila. (également disponible à l'adresse http://extwprlegs1.fao.org/docs/pdf/phi152934.pdf).

Climate Investment Fund. 2016. PPCR Monitoring and Reporting Toolkit. Pilot Programme for Climate Resilience. (également disponible à l'adresse https://portals.iucn.org/library/sites/library/files/documents/2006-041.pdf).

Climate Outreach and Adaptation Scotland. 2017. Communicating climate change adaptation. A practical guide to valuesbased communication. Adaptation Scotland/Government of Scotland, Edinburgh. (également disponible à l'adresse http://climateoutreach.org/resources/communicating-climate-change-adaptation-a-practical-guide-to-valuesbased-communication/).

Colls, A., Ash, N. et Ikkala, N. 2009. Ecosystem-based Adaptation: a natural response to climate change. Gland, Switzerland: IUCN. (également disponible à l'adresse https://www.iucn.org/content/ecosystem-based-adaptation-a-natural-response-climate-change). 
Crumpler, K., Bloise, M., Meybeck, A., Salvatore, M. et Bernoux, M. 2019. Linking nationally determined contributions and the sustainable development goals through agriculture: A methodological framework. Environment \& Natural Resources Management Working Paper No. 75, Rome, FAO. 40 pp. (également disponible à l'adresse http://www.fao.org/3/ca5003en/ca5003en.pdf).

D'Annunzio, R., Gianvenuti, A., Henry, M. et Thulstrup, A. 2016. Manual for assessing woodfuel supply and demand in displacement settings. Rome, FAO \& Haut Commissariat des Nations Unies pour les réfugiés (HCR).

Dasgupta, S., Hossain, M., Huq, M. et Wheeler, D. 2014. Climate change, soil salinity, and the economics of high-yield rice production in coastal Bangladesh. Document de recherche sur les politiques de la Banque mondiale $n^{\circ} 7140$. Washington, DC, Banque mondiale.

Dazé, A., Ambrose, K. et Ehrhart, C. 2009. Climate vulnerability and capacity analysis handbook. CARE International.

DCLG. 2009. Multi-criteria analysis: a manual. Department for Communities and Local Government, Londres. également disponible à l'adresse

https://assets.publishing.service.gov.uk/government/uploads/system/uploads/attachment_data/file/7612/1132618.pdf).

De Bruin, K., Dellink, R.B., Ruijs, A., Bolwidt, L., van Buuren, A., Graveland, J., de Groot, R.S., et al. 2009. Adapting to climate change in The Netherlands: an inventory of climate adaptation options and ranking of alternatives. Climatic Change, 95: 23-45. DOI 10.1007/s10584-009-9576-4. (également disponible à l'adresse http://link.springer.com/article/10.1007/s10584-009-9576-4).

Djoudi, H. et Brockhaus, $\mathbf{M}$. 2011. Is adaptation to climate change gender neutral? Lessons from communities dependent on livestock and forests in northern Mali. International Forestry Review, 13(2): 123-135.

Edwards, J.E., Pearce, C., Ogden, A.E., et Williamson, T.B. 2015. Climate change and sustainable forest management in Canada: a guidebook for assessing vulnerability and mainstreaming adaptation into decision making. Conseil canadien des ministres des forêts, Groupe de travail sur le changement climatique. Ottawa.

Fisher, R.J., Srimongkontip, S. et Veer, C. 1997. People and forests in Asia and the Pacific: situation and prospects. Document de travail FAO/RAPA APFSOS/WP/27. Bangkok, FAO.

FAO. 2006. Fire management Voluntary guidelines: Principles and strategic actions. Working Paper 17/E. Rome. (également disponible à l'adresse http://www.fao.org/3/j9255e/j9255eoo.pdf).

FAO. 2012. Mainstreaming Climate-Smart Agriculture into a Broader Landscape Approach. Rome. (également disponible à l'adresse http://www.fao.org/3/ap402e/ap402e.pdf).

FAO. 2013. Climate change guidelines for forest managers. FAO Forestry Paper No. 172. Rome.

FAO. 2014. Climate-Smart Agriculture Sourcebook. Rome. (également disponible à l'adresse http://www.fao.org/3/i3325e/i3325e.pdf).

FA0. 2016a. Climate change and food security: Risks and responses. Rome. également disponible à l'adresse http://www.fao.org/3/a-i5188e.pdf).

FAO. 2016b. The State of Food and Agriculture: Climate Change, Agriculture and Food Security. Rome. (également disponible à l'adresse http://www.fao.org/3/a-i6030e.pdf).

FAO. 2017a. Intended nationally determined contributions: global analysis key findings. Rome. (également disponible à l'adresse http://www.fao.org/3/a-i6573e.pdf).

FA0. 2017b. Sustainable woodfuel for food security A smart choice: green, renewable and affordable. Rome. (également disponible à l'adresse http://www.fao.org/3/a-i7917e.pdf)

FAO. 2017c. Tracking adaptation in agricultural sectors. Rome. (également disponible à l'adresse http://www.fao.org/3/a-i8145e.pdf).

FA0. 2018a. The State of the world's forests 2018. Forest pathways to sustainable development. Rome. (également disponible à l'adresse http://www.fao.org/3/I9535EN/i9535en.pdf).

FAO. 2018b. Institutional capacity assessment approach for national adaptation planning in the agriculture sectors. Note d'information. (également disponible à l'adresse http://www.fao.org/3/I8900EN/i890oen.pdf).

FA0. 2018c. Terms and Definitions FRA 2020. Forest Resources Assessment Working paper 188. Rome. (également disponible à l'adresse http://www.fao.org/3/I8661EN/i8661en.pdf).

FAO. 2018d. Guidelines and Specifications FRA 2020. Forest Resources Assessment Working paper 189. Rome. (également disponible à l'adresse http://www.fao.org/3/I8699EN/i8699en.pdf).

FA0. 2018e. Climate change for forest policy-makers - An approach for integrating climate change into national forest policy in support of sustainable forest management. Version 2.0. Document sur la foresterie $n^{\circ} .181$ de la FAO. (également disponible à l'adresse http://www.fao.org/3/CA2309EN/ca2309en.PDF). 
FAO. 2019. Regional Analysis of the Nationally Determined Contributions in Southern Europe, Eastern Europe and Central Asia Gaps and opportunities in the agriculture sectors. Rome. (également disponible à l'adresse http://www.fao.org/3/CA2518EN/ca2518en.pdf)

FA0. 2020a. Adaptation in the agriculture sectors: leveraging co-benefits for mitigation and sustainable development. Rome. (également disponible à l'adresse http://www.fao.org/3/ca9195en/ca9195en.pdf)

FA0. 2020b. Regional Analysis of the Nationally Determined Contributions in Asia. Rome. (également disponible à l'adresse http://www.fao.org/3/ca7264en/CA7264EN.pdf)

FAO et UNDP. 2018. Integrating Agriculture in National Adaptation Plans (NAP-Ag) Programme. Case study: the Philippines. Rome. (également disponible à l'adresse http://www.fao.org/3/CA3024EN/ca3024en.pdf).

FAO et UNDP. 2019. Enhancing adaptation planning for agricultural resilience in Viet Nam: Linkages between National Adaptation Plans (NAPs) and Nationally Determined Contributions (NDCs). Case Study. (également disponible à l'adresse https://www.vn.undp.org/content/dam/vietnam/docs/Publications/2019_UNDP_NAPNDC\%20Case\%20Study.pdf).

FAO et UNDP. 2020a. Integrating Agriculture in National Adaptation Plans Case Study: Uganda. Rome (également disponible à l'adresse http://www.fao.org/3/cb0576en/cb0576en.pdf)

FAO et UNDP. 2020b. Assessing sustainable water conservation and agroforestry practices for climate change adaptation in Kenya: A cost-benefit analysis. Roma.

Forest Management Bureau, Philippines. 2016. Master Plan for Climate Resilient Forestry Development. (également disponible à l'adresse https://forestry.denr.gov.ph/pdf/mp/PMPCRFD_2015_plus_Annexes.pdf).

Gaisberger, H., Kindt, R., Loo, J., Schmidt M., Bognounou, F., Da, S., Diallo, O.B., et al. 2017. Spatially explicit multithreat assessment of food tree species in Burkina Faso: a fine-scale approach. PLOS ONE, 12(9): e0184457.

Garrett, A., Buckley, P., Brown, S., et Townhill, B. 2015. Climate change adaptation in UK seafood: Understanding and responding to climate change in the UK wild capture seafood industry. A joint Seafish/MCCIP summary. Seafish, Edinburgh. doi:10.14465/2015.sarps.001-016. (également disponible à l'adresse http://www.seafish.org/media/1476670/climate_change_annex_lr2.pdf ).

Gebrekirstos, A., Bräuning, A., Sass-Klassen, U. et Mbow, C. 2014. Opportunities and applications of dendrochronology in Africa. Current Opinion in Environmental Sustainability, 6: 48-53.

GIZ et IISD. 2014. Monitoring and evaluating adaptation at aggregated levels: A comparative analysis of ten Systems. Deutsche Gesellschaft für Internationale Zusammenarbeit (GIZ), Bonn. (également disponible à l'adresse https://www.adaptationcommunity.net/download/me/me-guides-manuals-reports/GIZ_2014-Comparative_ analysis_of_national_adaptation_M\&E.pdf).

Global Commission on Adaptation. 2019. Adapt now: a global call for leadership on climate resilience. Global Center on Adaptation and World Resources Institute.

Gobierno de Chile. 2014. Plan Nacional de Adaptación al Cambio Climático - Elaborado en el marco del Plan de Acción Nacional de Cambio Climático. Chile. (également disponible à l'adresse http://portal.mma.gob.cl/wp-content/ uploads/2016/02/Plan-Nacional-Adaptacion-Cambio-Climatico-version-final.pdf).

Guariguata, M.R., Locatelli, B. et Haupt, F. 2012. Adapting tropical production forests to global climate change: risk perceptions and actions. International Forestry Review, 14(1): 27-38.

GWP. 2019. Addressing Water in National Adaptation Plans. (également disponible à l'adresse https://wwW4.unfccc.int/sites/NAPC/Documents/Supplements/GWP_NAP_Water_Supplement_May2019.pdf).

Hahn, M. et Fröde, A. 2011. Climate proofing for development. Adapting to climate change, reducing risk. Deutsche Gesellschaft für Internationale Zusammenarbeit (GIZ) GmbH, Eschborn. (également disponible à l'adresse http://www.preventionweb.net/files/globalplatform/entry_bg_paper giz2011climateproofing.pdf).

Head, B.W., Ross, H. et Bellamy, J. 2016. Managing wicked natural resource problems: The collaborative challenge at regional scales in Australia. Landscape and Urban Planning, 154: 81-92.

HLPE. 2017. Sustainable forestry for food security and nutrition. A report by the High-Level Panel of Experts on Food Security and Nutrition of the Committee on World Food Security. Rome. (également disponible à l'adresse http://www.fao.org/3/ai7395e.pdf).

Holzschuh, A., Steffan-Dewenter, I. et Tscharntke, T. 2010. How do landscape composition and configuration, organic farming and fallow strips affect the diversity of bees, wasps and their parasitoids? Journal of Animal Ecology, 79: 491-500.

Incropera, F.P. 2015. Climate Change: A Wicked Problem. Cambridge University Press, New York. 
IPCC. 2001. McCarthy, J.J,. Canziani, O.F, Leary, N.A., Dokken, D.J. et White, K.S, eds. Climate Change 2001: Impacts, Adaptation, and Vulnerability. Contribution of Working Group II to the Third Assessment Report of the Intergovernmental Panel on Climate Change Cambridge, UK, Cambridge University Press.

IPCC. 2007. Parry, M.L., Canziani, O.F, Palutikof, J.P., van der Linden, P.J. et Hansen, C.E., eds. Climate Change 2007: Impacts, adaptation and vulnerability. Contribution of Working Group II to the Fourth Assessment Report of the Intergovernmental Panel on Climate Change. Cambridge, UK, Cambridge University Press. (également disponible à l'adresse https://www.ipcc.ch/site/assets/uploads/2018/03/ar4_wg2_full_report.pdf).

IPCC. 2014. Climate change 2014: Impacts, adaptation, and vulnerability. Intergovernmental Panel on Climate Change, Working Group II. (également disponible à l'adresse http://www.ipcc.ch/report/ar5/wg2/).

IPCC. 2019. Special report on climate change and land. (également disponible à l'adresse https://www.ipcc.ch/srccl/).

IUCN. 2009b. No time to lose - make full use of nature-based solutions in the post-2012 climate change regime. Position paper on the Fifteenth session of the Conference of the Parties to the United Nations Framework Convention on Climate Change (COP 15). Gland: IUCN. (également disponible à l'adresse

https://www.iucn.org/sites/dev/files/import/downloads/iucn_position_paper_unfccc_cop_15.pdf).

Johnston, M., Williamson, T., Munson, A., Ogden, A., Moroni, M., Parsons, R., Price, D. et Stadt, J. 2010. Climate change and forest management in Canada: impacts, adaptive capacity and adaptation options - A State of Knowledge report. Sustainable Forest Management Network, Edmonton, Alberta. 54 pp.

Juergenliemk-Avagyan, A., Wolf, J., Kohli, R., Garcia, C., Spairani, A., Teng, J., Terrefe, D. et Hodes G. 2017. The role of the National Adaptation Plans (NAPS) in agriculture for achieving multiple Sustainable Development Goals and implementing the Paris Agreement. Proceedings of the International Conference on Sustainable Development.New York, USA.

Karttunen, K., Wolf, J., Garcia, C. et Meybeck, A. 2017. Addressing Agriculture, Forestry and Fisheries in National Adaptation Plans - Supplementary Guidelines. Rome, FAO. (également disponible à l'adresse http://www.fao.org/3/a-i6714e.pdf).

Katila, P., Pierce Colfer, C., De Jong, W., Galloway, G., Pacheco, P., et Winkel, G., eds. (2019). Sustainable Development Goals: Their Impacts on Forests and People. Cambridge: Cambridge University Press. doi:10.1017/9781108765015

Khadka, C., Hujala, T., Worlfslehner, B. et Vacik, H. 2013. Problem structuring in participatory forest planning. Forest Policy and Economics. 26: 1-11.

Koffi, C. K., Djoudi, H. et Gautier, D. Landscape diversity and associated coping strategies during food shortage periods: evidence from the Sudano-Sahelian region of Burkina Faso. Regional Environmental Change 17, no. 5 (2017): 1369-1380.

Lambrou, Y. et Piana, G. 2006. Gender: the missing component of the response to climate change. Roma, FAO.

LEG (Least Developed Countries Expert Group). 2012. National Adaptation Plans. Technical guidelines for the national adaptation plan process. UNFCCC Secretariat. Bonn. Germany. December 2012. (également disponible à l'adresse http://www4.unfccc.int/nap/Guidelines/Pages/Technical-guidelines.aspx).

LEG (Least Developed Countries Expert Group). 2013. Development of a tool for monitoring, reviewing and assessing progress, effectiveness and gaps under the NAP process in LDCs: the PEG M\&E Tool. By Latasi, P., UNFCCC Adaptation Committee workshop on the M\&E of adaptation 9-11 September 2013, Nadi, Fiji. (slides). (également disponible à l'adresse https://unfccc.int/files/adaptation/cancun_adaptation_framework/adaptation_committee/ application/pdf/peg_tool_latasi.pdf).

Lilles $\emptyset$, J. B. L., Graudal, L., Moestrup, S., Kjær, E.D., Kindt, R., Mbora, A., Dawson, I., et al. 2011. Innovation in input supply systems in smallholder agroforestry: seed sources, supply chains and support systems. Agroforest Syst (2011) 83:347-359 DOI 10.1007/s10457-011-9412-5

Lillesø, J.P.B., Harwood, C., Derero, A., Graudal, L., Roshetko, J. M., Kindt R., Moestrup S., et al. 2018. Why institutional environments for agroforestry seed systems matter. Dev Policy Rev. 2018; 36: 089-0112

Lim, B. et Spanger-Siegfred, E. (eds). 2005. Adaptation Policy Framework for Climate Change. Developing Strategies, Policies and Measures. Cambridge, UK, Cambridge University Press (également disponible à l'adresse www.preventionweb.net/publications/view/7995).

Locatelli, B., Kanninen, M., Brockhaus, M., Colfer, C.J.P., Murdiyarso, D. et Santoso, H. 2008 Facing an uncertain future: How forests and people can adapt to climate change. Forest Perspectives No. 5. CIFOR, Bogor, Indonesia.

Locatelli B., Pavageau C., Pramova E., Di Gregorio M., 2015. Integrating climate change mitigation and adaptation in agriculture and forestry: Opportunities and trade-offs. WIREs Climate Change, 6(6): 585-598.

http://onlinelibrary.wiley.com/doi/10.1002/wcc.357/pdf 
Locatelli B. 2016. Ecosystem services and climate change. M. Potschin, R., Haines-Young, R. Fish et R.K. Turner (eds) Routledge Handbook of Ecosystem Services. Routledge, London and New York, pp.481-490. (également disponible à l'adresse http://agents.cirad.fr/pjjimg/bruno.locatelli@cirad.fr/Locatelli_2016_Handbook_Ecosystem_Services_ and_Climate_Change_PostPrint.pdf).

Louman B., Meybeck A., Mulder G., Brady M., Fremy L., Savenije H., Gitz V. et Trines E. 2020. Scaling of innovative finance for sustainable landscapes. FTA.

Lumosi, C. et McGahey, D. 2016. Communicating climate change for adaptation: Challenges, successes and future priorities. Information Brief. Collaborative Adaptation Research Initiative in Africa and Asia (CARIAA). International Development Research Centre, Ottawa. (également disponible à l'adresse

https://www.weadapt.org/knowledge-base/assar/communicating-climate-change-for-adaptation).

Lundgren, B.O. et Raintree, J.B. 1982. Sustained agroforestry. In Nestel, B. ed. Agricultural research for development: potentials and challenges in Asia, pp. 37-49. The Hague, ISNAR.

MAAIF. 2018. National Adaptation Plan for the Agricultural Sectors in Uganda. Kampala. (également disponible à l'adresse https://www.agriculture.go.ug/wp-content/uploads/2019/09/National-Adaptation-Plan-for-the-Agriculture-Sector-1.pdf).

Mackay, A., Gallo, I., Husch, J. et Raksakulthai, V. 2015. Skills Assessment for National Adaptation Planning. How countries can identify the gap. United Nations Institute for Training and Research (UNITAR), Geneva. également disponible à l'adresse http://www.uncclearn.org/sites/default/files/inventory/nap16062015.pdf).

MacKinnon, K., Sobrevila, C., Hickey, V. 2008. Biodiversity, climate change, and adaptation: nature-based solutions from the World Bank portfolio (English). Washington, DC: Banque mondiale. http://documents.worldbank.org/curated/ en/149141468320661795/Biodiversity-climate-change-and-adaptation-nature-based-solutions-from-the-WorldBank-portfolio.

McNaught, R., Warrick, O. et Cooper, A. 2014. Communicating climate change for adaptation in rural communities: a Pacific study. Regional Environmental Change, 14:1491-1503.

Meybeck, A., Rose, S. et Gitz, V. 2019. Climate change vulnerability assessment of forests and forest-dependent people - A framework methodology. Document sur la foresterie $n^{\circ}$. 183. Rome, FAO.

Metzger, M.J. et Schröter, D. 2006. Towards a spatially explicit and quantitative vulnerability assessment of environmental change in Europe. Regional Environmental Change, 6(4): 201-216.

Ministère de l'élevage, de l'agriculture et de la pêche (MGAP). 2019. National Adaptation Plan for the Agriculture Sector (PNA-Agro). Uruguay. (également disponible à l'adresse https://www4.unfccc.int/sites/NAPC/Documents/Parties/NAP\%20Agriculture\%20Uruguay.pdf).

Moore, W., Alleyne, F., Alleyne, Y., Blackman, K., Blenman, C., Carter, S., Cashman, A., et al. 2014. Barbados' Green Economy Scoping Study. Government of Barbados, University of West Indies - Cave Hill Campus, United Nations Environment Programme (UNEP). (également disponible à l'adresse

http://www.greengrowthknowledge.org/resource/green-economy-scoping-study-synthesis-report-barbados).

Morin, X., Fahse, L., Jactel, H., Scherer-Lorenzen, M., García-Valdés, R. y Bugmann, H., 2018. Long-term response of forest productivity to climate change is mostly driven by change in tree species composition. Scientific reports, 8(1): 5627.

Newton, P., Miller, D. C., Ateenyi Byenkya, M. A., Agrawal A. 2016. Who are forest-dependent people? A taxonomy to aid livelihood and land use decision-making in forested regions. Land Use Policy 57: 388-395.

Ndlovu, E., 2013. Impact of tree species on maize productivity by smallholder farmers in Eastern Kenya. Unpublished Master Thesis, Jomo Kenyatta University of Agriculture and Technology, Juja, Kenia.

Nyoka, B. I., Roshetko, J., Jamnadass, R., Kalinganire, A., Lilles J. B. L., Beedy T., Cornelius J. 2014. Tree Seed and Seedling Supply Systems: A Review of the Asia, Africa and Latin America Models. Small-scale Forestry. DOI 10.1007/ s11842-014-9280-8

Obeng, E.A., Owusu-Sekyere, E., Dumenu, W.K., Nutakor, E.E., Samar, S.B. et Opoku, E.A. 2011. Climate change and forest dependent livelihoods: vulnerabilities and impacts. Technical report, Government of Ghana Project.

Parkins, J.R. et MacKendrick, N.A. 2007. Assessing community vulnerability: a study of the mountain pine beetle outbreak in British Columbia, Canada. Global Environmental Change 17(3): 460-471.

Porter, J.R., L. Xie, Challinor, A.J., Cochrane, K., Howden, S.M., Iqbal, M.M., Lobell, D.B. et Travasso, M.I. 2014. Food security and food production systems. In: Climate Change 2014: Impacts, Adaptation, and Vulnerability. Part A: Global and Sectoral Aspects. Contribution of Working Group II to the Fifth Assessment Report of the Intergovernmental Panel on Climate Change [Field, C.B., Barros, V.R., Dokken, D.J., Mach, K.J., Mastrandrea, M.D., Bilir, T.E., Chatterjee, M., et al., (eds.)]. Cambridge University Press, Cambridge, United Kingdom and New York, NY, USA, pp. 485-533. 
Pramova, E., Locatelli, B., Brockhaus, M., Fohlmeister, S., 2012. Ecosystem services in the National Adaptation Programmes of Action. Climate Policy 12(4): 393-409. (également disponible à l'adresse https://www.tandfonline.com/doi/pdf/10.1080/14693062.2011.647848).

Pramova, E., Locatelli, B., Djoudi, H., Somorin, O., 2012. Forests and trees for social adaptation to climate variability and change. WIREs Climate Change 3: 581-596. (également disponible à l'adresse http://onlinelibrary.wiley.com/doi/10.1002/wcc.195/pdf)

Price-Kelly, H. 2016. Financing National Adaptation Plans (NAP): Options for implementation. Targeted Topics Forum Report 2. International Institute for Sustainable Development, Winnipeg. (également disponible à l'adresse http://www.napglobalnetwork.org/wp-content/uploads/2016/05/TTF2-Report.pdf).

Price-Kelly, H. et Hammill, A. 2016. sNAPhot: Domestic public finance for implementation of NAP. Overview Brief II. NAP Global Network Secretariat, International Institute for Sustainable Development, Winnipeg. également disponible à l'adresse http://napglobalnetwork.org/resource/snapshot-domestic-public-finance-implementation-naps/).

Pureswaran, Deepa S., Alain Roques, et Andrea Battisti. Forest insects and climate change. Current Forestry Reports, 4, no. 2 (2018): 35-50.

Puri, J. (2018). Transformational Change - The Challenge of a Brave New World. Independent Evaluation Unit (IEU) Learning Paper No. 1, Green Climate Fund. Songdo, South Korea.

Ranjitkar, S., Sujakhu, N.M., Merz, J., Kindt, R., Xu, J., Matin, M.A., Ali, M. et Zomer, R.J. 2016a. Suitability analysis and projected climate change impact on banana and coffee production zones in Nepal. PLOS One, 11(9): e0163916.

Ranjitkar, S., Sujakhu, N.M., Lu, Y., Wang, Q., Wang, M., He, J., Mortimer, P.E., Xu, J., Kindt, R. et Zomer, R.J. 2016 b. Climate modelling for agroforestry species selection in Yunnan Province, China. Environmental Modelling \& Software, 75: 263-272.

Ranjitkar, S., Sujakhu, N.M., Merz, J., Kindt, R., Xu, J., Matin, M.A., Ali, M. et Zomer, R.J. 2016a. Suitability analysis and projected climate change impact on banana and coffee production zones in Nepal. PLOS One, 11(9): e0163916.

Reid, H., Hou Jones, X., Porras, I., Hicks, C., Wicander S., Seddon, N., Kapos V., Rizvi A.R. et Roe, D. 2019. Is Ecosystem-based adaptation effective? Perceptions and lessons learned from 13 project sites. IIED Research Report. IIED, London. ((également disponible à l'adresse https://pubs.iied.org/17651IIED/).

Ricketts, T.H. 2004. Tropical forest fragments enhance pollinator activity in nearby coffee crops. Conservation Biology, 18(5): 1262-1271.

Ricketts, T.H., Regetz, J., Steffan-Dewenter, I., Cunningham, S.A., Kremen, C., Bogdanski, A., Gemmill-Herren, B., Greenleaf, S.S., Klein, A.M., Mayfield, M.M., Morandin, L.A., Ochieng, A. et Viana B.F. 2008. Landscape effects on crop pollination services: are there general patterns? Ecology Letters, 11: 499-515.

Rodenberg, B. 2009. Climate change adaptation from a gender perspective: a cross-cutting analysis of development-policy instruments. Discussion Paper 24/2009. Bonn, Germany, German Development Institute (DIE).

Roques, A. et al. 2015. Climate Warming and Past and Present Distribution of the Processionary Moths (Thaumetopoea spp.) in Europe, Asia Minor and North Africa. In: Roques A. (eds) Processionary Moths and Climate Change: An Update. Springer, Dordrecht

Scienseed. 2016. Communicating climate change and biodiversity to policy makers. A guide by Scienseed SL for the Bern Convention (Council of Europe). (également disponible à l'adresse https://rm.coe.int/168064e897).

Shackleton C. et Shackleton, S. 2004. The importance of non-timber forest products in rural livelihood security and as safety nets: a review of evidence from South Africa. South African Journal of Science, 100, November/December 2004.

Southern Voices on Adaptation, 2018. NAP progress in participation and transparency, assessment by SV-Adapt partner networks in Vietnam, Cambodia, Nepal, Sri Lanka, Kenya, and Malawi. (également disponible à l'adresse http://unfccc.int/files/adaptation/workstreams/national_adaptation_plans/application/pdf/participation_and_ transparency_by_svadapt.pdf).

Spearman, M. et McGray, H. 2011. Making adaptation count. Concepts and options for monitoring and evaluation of climate change adaptation. GIZ and World Resources Institute (WRI), Washington D.C. (également disponible à l'adresse http://www.wri.org/publication/making-adaptation-count).

Stevens-Rumann, Camille S., Kemp, Kerry B., Higuera, Philip E., Harvey, Brian J., Rother Monica T., Donato, Daniel C., Morgan, P., et Veblen. T.T. Evidence for declining forest resilience to wildfires under climate change. Ecology Letters 21, no. 2 (2018): 243-252.

Strohmaier, R., Rioux, J., Seggel, A., Meybeck, A., Bernoux, M., Salvatore, M., Miranda, J. et Agostini, A. 2016. The 
Agriculture sectors in the Intended Nationally Determined Contributions: Analysis. Environment and Natural Resources Management Working Paper No. 62. Rome, FAO. (également disponible à l'adresse http://www.fao.org/3/a-i5687e.pdf).

Sunderland, T., Achdiawan, R., Angelsen, A., Babigumira, R., Ickowitz, A., Paumgarten, F., Reyes-Garcia, V. et Shively, G. 2014. Challenging perceptions about men, women, and forest product use: a global comparative study. World Development, (64, Suppl. 1): 56-66.

Termeer, C., Dewulf, A. et Breeman, G. 2013. Governance of wicked climate adaptation problems. In: J. Knieling and W.L. Filho (eds.) Climate Change Governance. Climate Change Management, DOI: 10.1007/978-3-642-29831-8_3, Springer-Verlag, Berlin.

UNDP. 2018. Viet Nam's Climate Risk Index. http://eng.climaterisk.org.vn/

UNEP. 2012. Ecosystem-based adaptation guidance. Moving from principles to practice. Working Document: April 2012. Geneva. (également disponible à l'adresse http://www.unep.org/).

United Nations Forum on Forests (UNFF) et Collaborative Partnership on Forests (CPF). 2018. Draft of Global Core Sets. (également disponible à l'adresse http://www.cpfweb.org/48715-0dfc673f9958ed5b6909f2e5b45b711fc.pdf).

UNFCCC. 2011a. Ecosystem-based approaches to adaptation: compilation of information. Note by the secretariat. Subsidiary Body for Scientific and Technological Advice, Thirty-fifth session, Durban, 28 November to 3 December 2011, FCCC/ SBSTA/2011/INF.8. (également disponible à l'adresse www.unfccc.int/resource/docs/2011/sbsta/eng/info8.pdf).

UNFCCC. 2011b. Assessing the costs and benefits of adaptation options: An overview of approaches. UNFCCC Secretariat, Bonn. (également disponible à l'adresse http://unfccc.int/files/adaptation/nairobi_work_programme/knowledge_ resources_and_publications/application/pdf/2011_nwp_costs_benefits_adaptation.pdf).

UNFCCC. 2015. Synthesis report on the aggregate effect of the intended nationally determined contributions. Note by the secretariat. FCCC/CP/2015/7, 30 October 2015.(également disponible à l'adresse https://unfccc.int/resource/docs/2015/cop21/eng/07.pdf).

UNFCCC. 2017. The 31st meeting of the Least Developed Countries Expert Group Report by the secretariat. FCCC/ $\mathrm{SBI} / 2017 / 6$

UNFCCC. 2018. Progress, experience, best practices, lessons learned, gaps, needs and support provided and received in the process to formulate and implement national adaptation plans. Synthesis report by the secretariat. FCCC/SBI/2018/INF.1

UNISDR (United Nations International Strategy for Disaster Reduction). 2009. UNISDR terminology on disaster risk reduction. Geneva (également disponible à l'adresse www.unisdr.org/we/inform/terminology).

USAID, 2013. Analysing climate change adaptation options using multi-criteria analysis. African and Latin American Resilience to Climate Change (ARCC). US Agency for International Development, Washington, D.C. (également disponible à l'adresse http://www.iee.usp.br/sites/default/files/Analyzing_climate_change_adaptation_options using_multi-criteria_analysis_2013_o.pdf).

UN-Water. 2014. International Decade for Action 'Water for Life, 2005-2015'.

van Breugel, P., Friis, I., Demissew, S., Lilles $\emptyset$, J.-P.B. et Kindt, R. 2016. Current and future fire regimes and their influence on natural vegetation in Ethiopia. Ecosystems, 19(2): 369-386.

Viggh, A., Leagnavar, P. et Bours, D. 2015. Good practice study on principles for indicator development, selection, and use in climate change adaptation monitoring and evaluation. Climate-Eval. GEF Independent Office of Evaluation, GEF, Ginebra. (également disponible à l'adresse https://www.climate-eval.org/sites/default/files/studies/Good-Practice-Study.pdf).

Vira, B., Wildburger, C. et Mansourian, S., eds. 2015. Forests, trees and landscapes for food security and nutrition. IUFRO World Series, 33.

Watkiss, P. et Hunt, A. 2013. Method overview: Decision support methods for adaptation. Briefing Note 1. Summary of Methods and Case Study Examples from the MEDIATION Project. Funded by the EC's 7FWP. (également disponible à l'adresse https://mediamanager.sei.org/documents/Publications/sei-mediation-briefing1-method-overview.pdf).

WRI. 2018. Connecting the Dots: Elements for a Joined-Up Implementation of the 2030 Agenda and Paris Agreement

Yearworth, M. 2015. Sustainability as a "super wicked" problem; opportunities and limits for engineering methodology. In Proceedings of the 7th International Conference on Sustainable Development on Building and Environment (SuDBE). SuDBE. University of Bristol, UK. 



\section{INTÉGRATON DE LAFORESTERIE ET DE LAGROFORESTERIEDANS LESPLANS DADAPTATION NATIONAUX}

\section{[ Directives complémentaires ]}

Cette publication «Intégration de la foresterie et de l'agroforesterie dans les plans d'adaptation nationaux: directives complémentaires» vise à fournir des conseils techniques sur l'intégration des forêts, de l'agroforesterie et des arbres dans la formulation et l'implémentation des Plans d'Adaptation Nationaux. Elle a le but d'informer les représentants et les agents gouvernementaux, les responsables de la planification des Plans

d'Adaptation Nationaux, et tous les acteurs qui s'intéressent aux forêts, à

la foresterie et à l'agroforesterie en ce qui concerne l'adaptation et leurs potentiel support à l'adaptation d'autres secteurs, sous-secteurs, et activités. 